\title{
The Undeciphered Signs of Linear B
}

\author{
Anna Penelope Judson
}

\author{
Pembroke College
}

University of Cambridge

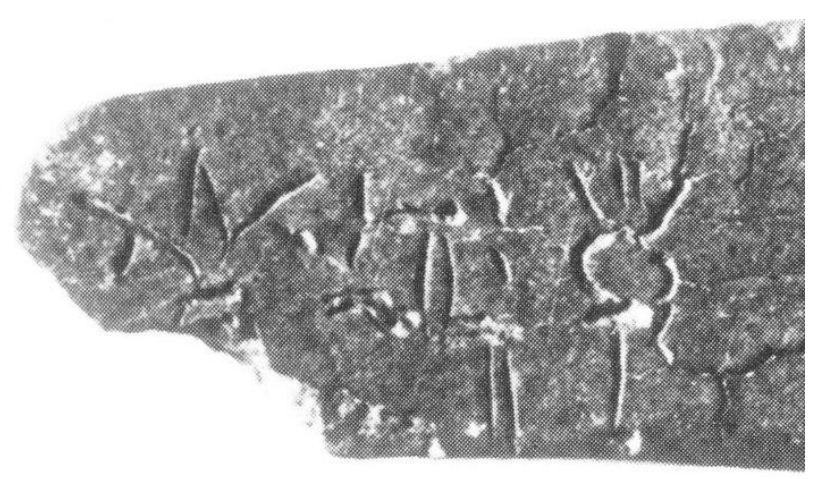

April 2016

This dissertation is submitted for the degree of Doctor of Philosophy. 



\section{Anna Penelope Judson, 'The Undeciphered Signs of Linear B'}

More than sixty years after Michael Ventris' decipherment of the Linear B script, 14 of its 87 syllabic signs still have no sound-values assigned to them. This group of 'undeciphered' signs represent a significant gap in our ability not only to read the Linear B script, but also to understand its development and use. Chapter 1 of this thesis analyses the origins and usage of signs with known sound-values to establish what types of values are in principle most likely to be found amongst the undeciphered signs: this investigation also enables an exploration of the development of Linear B, its relationship with its parent script Linear A, and the motivating factors underlying the creation of new Linear B signs. Chapter 2 consists of studies of each individual undeciphered sign, including a palaeographic analysis of their forms, discussion of their corpus of attestations, and an examination of their prospects of decipherment and possible soundvalues in the light of the results of Chapter 1. Finally, Chapter 3 employs this group of signs in a case-study to explore the potential of palaeographic analysis to contribute to our understanding of wider issues concerning the Linear B script and its context of use within the Mycenaean palaces. This case-study focuses in particular on two main uses of palaeography: as a means of assigning a relative chronology to Linear B texts, and as evidence for the reconstruction of the Mycenaean scribes' administrative work and training. 



\section{$\underline{\text { Preface }}$}

This dissertation is the result of my own work and includes nothing which is the outcome of work done in collaboration except as declared in the Preface and specified in the text.

Parts of the section on sign *34 in Chapter 2 are based on an essay (entitled 'The undeciphered sign *34/*35 and the direction of writing of Linear B') which was submitted to the University of Cambridge in February 2012 as part of the M.Phil. degree; these parts are included here due to their necessity for a complete discussion of the undeciphered signs. With this exception, this dissertation is not substantially the same as any that I have submitted, or that is being concurrently submitted, for a degree or diploma or other qualification at the University of Cambridge or any other University or similar institution except as declared in the Preface and specified in the text. I further state that no substantial part of my dissertation has already been submitted, or is being concurrently submitted, for any such degree, diploma or other qualification at the University of Cambridge or any other University or similar institution except as declared in the Preface and specified in the text.

This dissertation does not exceed the prescribed word limit of 80,000 words for the Degree Committee of the Faculty of Classics. It includes a CD containing two catalogues of data, as approved by the Student Registry. 


\section{$\underline{\text { Acknowledgements }}$}

I am deeply grateful for all of the financial assistance I have received to support the writing of this thesis, including an Arts and Humanities Research Council Doctoral Award (grant number AH/J500094/1; 2012-2015), the British Federation of Women Graduates' J. Barbara Northend Scholarship (2015-2016), and a grant from the Graduate Studies Fund of the Faculty of Classics, University of Cambridge (2015), as well as generous travel-related funding from the Faculty of Classics' Henry Arthur Thomas Travel Awards and Pembroke College's Graduate Student Support Fund and Scholarship Trust Fund.

My research has benefited greatly from being able to study selected Linear B tablets in person: I am grateful to the National Archaeological Museum of Athens, the Heraklion Archaeological Museum, the Ephorate of Antiquities of Boeotia, and the Ephorate of Antiquities of Chania for issuing me with permits for this purpose, and to all the museum staff who assisted me during my visits. In particular, I would like to thank Katerina Kostandi, Eleni Konstantinidi, and Euridike Velalopoulou in Athens, Georgia Flouda in Heraklion, Anna Mylona in Chania, and Ioannis Fappas in Thebes. I would also like to thank the British School at Athens, and especially Tania Gerousi, for their assistance with permit applications and their hospitality during my visits to Greece, and to thank Vassilis Petrakis for his helpful advice.

Especial thanks are due to my supervisor, Torsten Meißner, for his constant help and support, without which this thesis could not have been written; and to my examiners, John Bennet and Rupert Thompson, for their many helpful comments and a very beneficial discussion during the viva. I would also like to thank John Killen, the other 
members of the Mycenaean Epigraphy Group and the E Caucus in the Cambridge Faculty of Classics, and the many other friends and colleagues with whom I have discussed aspects of my work.

Finally, the greatest thanks go to my parents, Jenny and Lindsay, and my sister, Ellen: this thesis is dedicated to them, with love. 


\section{$\underline{\text { Abbreviations used in the text }}$}

For bibliographic abbreviations, see p.256ff.

For abbreviations used in the accompanying catalogues of data, see the "Notes on catalogues of data' included on the enclosed CD.

$\mathrm{AC}=$ Archives Complex (Pylos)

acc. $=$ accusative

$\mathrm{AP}=$ Apodoulou

$\mathrm{Ar}=$ Arsenal (Thebes)

$\mathrm{ARKH}=$ Arkhanes

$\mathrm{C}=$ consonant

$\mathrm{CH}=$ Cretan Hieroglyphic

CIPEM = Comité international permanent des études mycéniennes

$\mathrm{CR}=$ Crete

CS = Cypriot Syllabary

dat. $=$ dative

$\mathrm{EL}=$ Eleusis

$\mathrm{Ep}=$ Odos Epameinondou (Thebes)

EW $=$ East Wing (Knossos)

$\mathrm{f}$. = feminine

gen. $=$ genitive

$\mathrm{H}=$ Hand (designating a scribe)

HAM = object in the Heraklion Archaeological Museum (image credits)

HOM = House of the Oil Merchant (Mycenae)

HS = House of the Sphinx (Mycenae)

HT = Haghia Triada

$\mathrm{HV}=$ Ayios Vasileios

IE = Indo-European

$\mathrm{IK}=\mathrm{Iklaina}$

instr. $=$ instrumental

$\mathrm{IO}=$ Iouktas

ISJ = inscribed stirrup jar

$\mathrm{KH}=\mathrm{Khania}$

$\mathrm{KN}=\mathrm{Knossos}$ 
$\mathrm{KO}=$ Kophinas

$\mathrm{KR}=$ Kreusis

$\mathrm{LA}=$ Linear $\mathrm{A}$

$\mathrm{LB}=$ Linear $\mathrm{B}$

LBA = Late Bronze Age

LH = Late Helladic (chronological period)

loc. $=$ locative

LM = Late Minoan (chronological period)

$\mathrm{m} .=$ masculine

MER = from the collections of the Mycenaean Epigraphy Room, Faculty of Classics, Cambridge (image credits)

MI $=$ Midea

MIL = Miletus

$\mathrm{MM}=$ Middle Minoan (chronological period)

$\mathrm{MN}=$ man's name

$\mathrm{MY}=$ Mycenae

n. $=$ neuter

NAM = object in the National Archaeological Museum, Athens (image credits)

$\mathrm{NEB}=$ North Eastern Building (Pylos)

$\mathrm{NEP}=$ Northern Entrance Passage (Knossos)

NES = North-East Slope of acropolis (Mycenae)

nom. $=$ nominative

$\mathrm{O}=$ Odos Oidipodos (Thebes)

$\mathrm{P}=$ Odos Pelopidou (Thebes)

PA = Papoura

$\mathrm{PE}=$ Petras

$\mathrm{PH}=$ Phaistos

$\mathrm{PL}=$ Platanos

pl. $=$ plural

$\mathrm{PN}=$ personal name

$\mathrm{PO}=$ Poros

$\mathrm{PY}=$ Pylos

$\mathrm{R}=$ resonant

RCT $=$ Room of the Chariot Tablets, Knossos 
$\mathrm{RP}=$ Room of Pithoi (Thebes)

sg. = singular

SKO = Skoteino Cave

$\mathrm{SY}=$ Syme

$\mathrm{TC}=$ Treasure Chamber (Thebes)

$\mathrm{TH}=$ Thebes

THE $=$ Thera

$\mathrm{TN}=$ toponym

$\mathrm{V}=$ vowel

$\mathrm{VRY}=$ Vrysinas

$\mathrm{WH}=$ West House (Mycenae)

$\mathrm{WN}=$ woman's name

$\mathrm{WW}=$ West Wing (Knossos)

$\mathrm{ZA}=\mathrm{Zakros}$ 


\section{$\underline{\text { Table of contents }}$}

Introduction: The undeciphered signs of Linear B.

p. 1

Chapter 1: The possible values of the undeciphered signs: evidence from the structure and development of the Linear B script............................... p.5

1.1 Missing 'core' signs? ........................................................................... p.9

1.2 The 'extra' signs ................................................................................. p.13

1.2.1 Vowels ...................................................................................................... p.

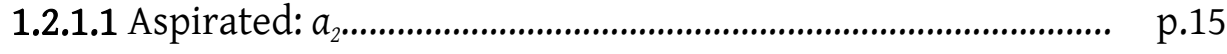

1.2.1.2 Diphthongs: $a_{3}, r a_{3}, a u$................................................................ p.17

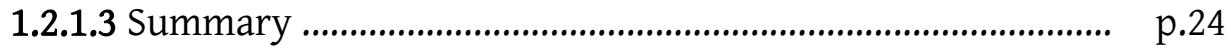

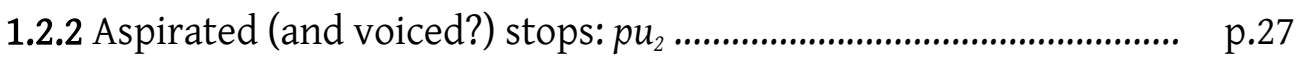

1.2.3 Labialised consonants ................................................................. p. p.34

1.2.3.1 Dentals: $d w e, d w o$, twe two ................................................. p... p. 34

1.2.3.2 Nasals: $n w a$......................................................................... p.42

1.2.3.3 Summary .................................................................................... p.

1.2.4 Palatalised consonants .................................................................. p.48

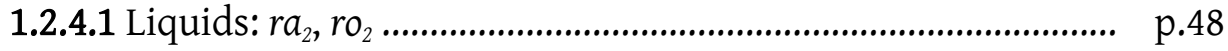

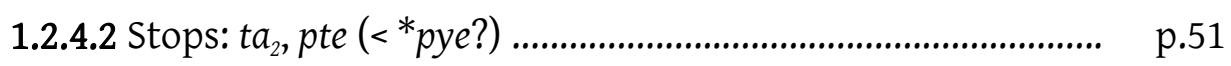

1.2.4.3 Summary …............................................................................. p.

1.3 Conclusions ................................................................................... p.63

Chapter 2: The undeciphered signs - individual studies ..................................... p.69

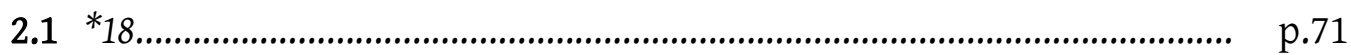

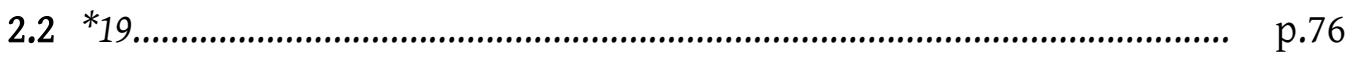

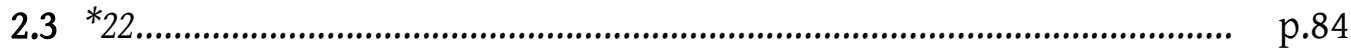

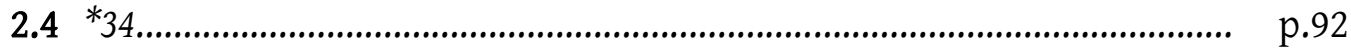

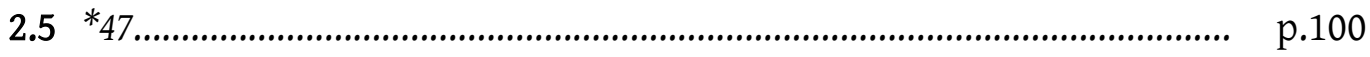

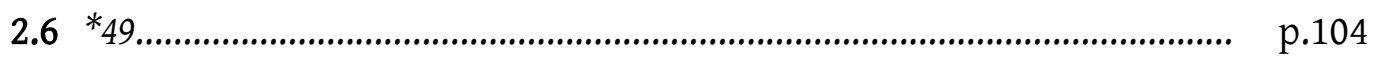

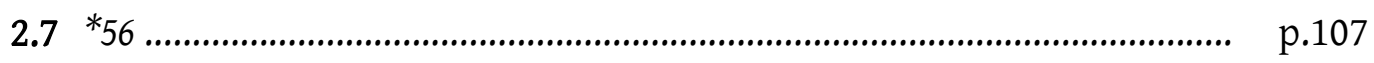

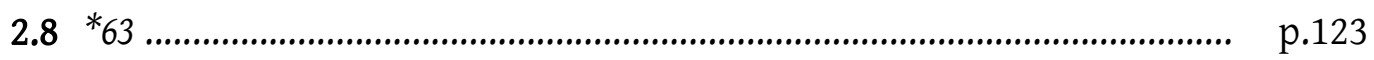

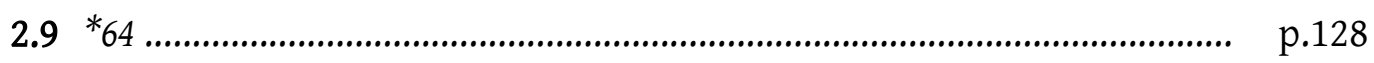

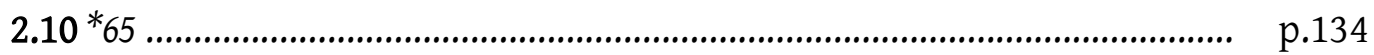

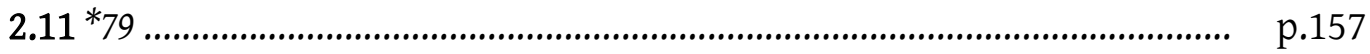

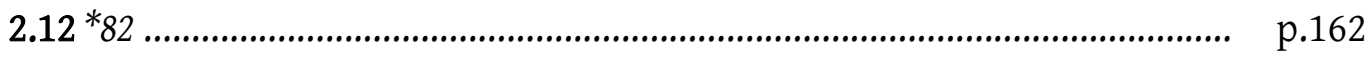

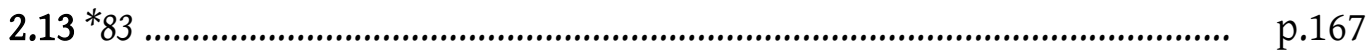

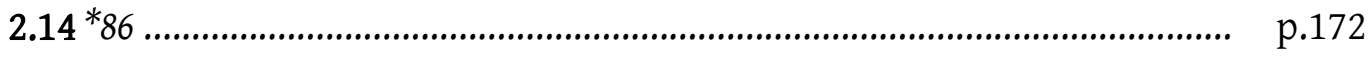

2.15 Conclusions ......................................................................................... p.174 
Chapter 3: The undeciphered signs as a palaeographic case-study ................... p.176

3.1 The Mycenaean scribes: administrative work and scribal training ..... p.176

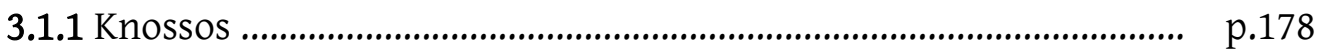

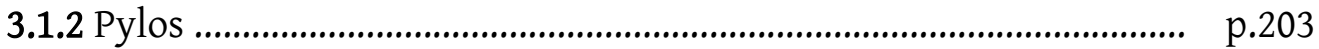

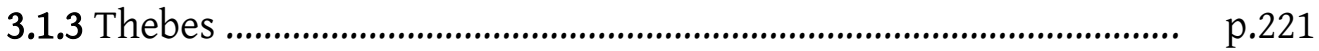

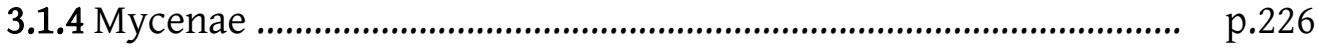

3.1.5 Conclusions …................................................................................. p. p.29

3.2 Script development and chronology ................................................... p.231

3.2.1 Knossian internal chronology ..................................................... p.233

3.2.2 Inter-site relative chronology and script development ................ p.241

3.2.3 Conclusions ....................................................................................... p. p.251

3.3 Case-study conclusions..................................................................... p.251

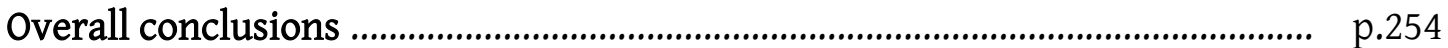

Bibliographic abbreviations......................................................................... p.256

Other bibliography................................................................................................. p.259 


\section{The undeciphered signs of Linear B}

\section{Introduction}

When Michael Ventris announced his decipherment of Linear B (LB) in July 1952, he had in fact assigned sound-values to around two-thirds of the script's syllabic signs; ${ }^{1}$ by the time of his publication of the decipherment the following year in collaboration with John Chadwick, this number had increased, but still only to around three-quarters of the syllabary. ${ }^{2}$ Subsequent work by Ventris, Chadwick, and other Mycenologists enabled some of these original sound-values to be modified and several more values to be identified; ${ }^{3}$ but more than sixty years after Ventris' decipherment, 14 of the $87 \mathrm{LB}$ syllabic signs - that is, around one-sixth of the syllabary - still have no sound-value

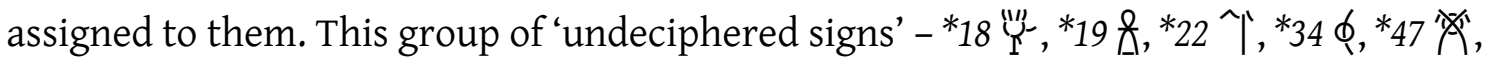

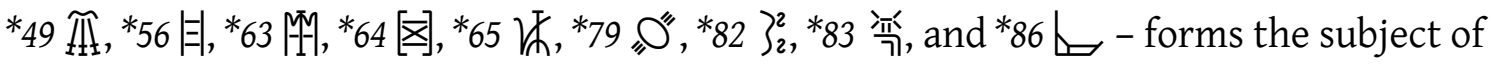
this thesis. ${ }^{4}$

In reality, the term 'undeciphered' covers a wide range of situations, since the actual status of these signs varies considerably: some are relatively frequent, others extremely rare; some are only certainly found at a single site, while others appear at multiple sites; a minority have proposed sound-values that have been relatively widely accepted, while others' possible values are much more uncertain or controversial. The contrast between

\footnotetext{
${ }^{1}$ Ventris 1952 [unpublished]:12, 'Experimental Syllabary'.

${ }^{2}$ Ventris-Chadwick 1953:86, fig.2.

${ }^{3}$ For a list and references, see Melena 2014b:13, fig.17.3.

${ }^{4}$ Excluded from this list are ${ }^{*} 89$, which is found only on KN Z 1715 and is probably a pseudo-sign (Judson 2013:80), and ${ }^{*} 92$, which has been proposed as a designation for two different unidentified signs, found in

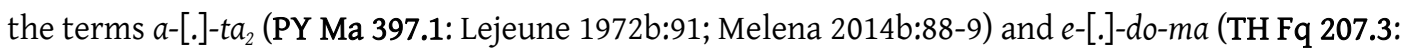
$T_{F C}{ }^{1}: 361$; Witczak 2002-2003). The latter can plausibly be read as - qa- (Palaima 2000-2001:484); the former is indeed unidentifiable, but this is due to the condition of the tablet, which makes it impossible to confirm whether it is in fact an otherwise unknown sign.
} 
signs such as ${ }^{*} 56$ (with up to 100 attestations on tablets from four different Cretan and mainland sites, as well as ISJs, and widely - though not universally - regarded as having the value $p a_{3}$ ) and ${ }^{*} 49$ (with no more than six attestations, all of which are at Knossos, and no generally accepted sound-value) is considerable. In fact, this group of signs is united only by the fact that, despite a large number of attempts at deciphering one or more of them, none has yet been the subject of a proposal sufficiently convincing for it to be considered 'deciphered' and provided with an official transcription by CIPEM. ${ }^{5}$ Nonetheless, considered together these signs represent a significant gap in our ability not only to read LB, but also to understand the history of its development; studying them as a group therefore provides an opportunity to examine their place in the LB script and their potential to contribute to a wider understanding of its use, as well as to analyse their individual properties and prospects of decipherment.

Chapter 1, 'The possible values of the undeciphered signs: evidence from the structure and development of the Linear B script', therefore takes a new approach to identifying these signs' possible values, analysing the origins and usage of signs with known soundvalues (in particular the 'extra' signs) to establish what types of values are in principle most likely to be found amongst the undeciphered signs. This analysis also enables an exploration of the development of LB, its relationship with its parent script Linear A (LA), and the motivating factors underlying the creation of new LB signs.

\footnotetext{
${ }^{5}$ It has often been argued that at least some of the undeciphered signs have a special status in representing non-Greek phonemes; I shall demonstrate, however, that there is no sufficient reason to assume this to be the case for the majority of these signs (see pp.70-1).
} 
Chapter 2, 'The undeciphered signs - individual studies', follows a more 'traditional' approach to these signs in some respects: a palaeographic analysis of the forms and attestations of each individual sign is followed by a discussion of its prospects of decipherment. A realistic examination of each sign's status in this regard is much needed given the current existence of numerous competing decipherment proposals for many of these signs. However, rather than presenting full decipherment proposals - which in most cases is not yet possible - this chapter focuses on combining evidence from each sign's corpus of attestations with the results of Chapter 1 in order to show how much currently can (or cannot) be relatively firmly established about the sign's possible sound-value(s), and therefore to provide as secure a basis as possible for any future decipherments.

Finally, Chapter 3, 'The undeciphered signs as a palaeographic case-study', employs the detailed palaeographic analyses carried out in Chapter 2 to look at the broader context of the LB script's use. The undeciphered signs are used in a case-study investigating two main possible uses of palaeography: as a means of dating LB texts (and hence the sites in which these texts are found), and as evidence for the reconstruction of the Mycenaean scribes' administrative work and training. This group of currently unreadable signs is therefore used to demonstrate the crucial potential of palaeography - a means of studying the script which does not rely on reading it - to contribute to our understanding of wider issues relating to LB's context of use in the Mycenaean palaces. 


\section{Note on citations}

Unless otherwise indicated, readings of $\mathrm{CH}$, LA, and LB texts and other related information (e.g. scribal hand attribution) follow the main corpora and scribal publications for each script or site; ${ }^{6}$ interpretations of LB terms follow DMic and (for phonetic interpretations of morphological elements) Bartoněk 2003. Further details relating to attestations of the LB extra and undeciphered signs are given in the catalogues on the accompanying CD (included at the back of the thesis).

Images reproduced from published works are cited as such in the text; other images are from the collections of the Mycenaean Epigraphy Group, Faculty of Classics, Cambridge (cited as 'MER') or were taken by the author during museum visits and included here by kind permission of the museums. These are cited as 'author (NAM)' (for objects in the National Archaeological Museum of Athens) or 'author (HAM)' (for objects in the Heraklion Archaeological Museum). Images are not reproduced to scale.

\footnotetext{
${ }^{6}$ CH: CHIC. LA: GORILA. LB: Ayios Vasileios: Aravantinos-Vasilogamvrou 2012. Iklaina: Shelmerdine 2012. ISJs: CIV; E. Hallager 2011. Khania: E. Hallager 2011. Knossos: COMIK; KT ${ }^{5}$; Olivier 1967; Driessen 2000. Mycenae: SCM; TITHEMY; Iakovidis et al. 2012. Pylos: PTT; PoNIV (though note that this is currently published only in preliminary form); Palaima 1988b. Thebes: TITHEMY; Piteros et al. 1990; TFC; Aravantinos et al. 2008. Tiryns: TITHEMY.
} 
Chapter 1: The possible values of the undeciphered signs: evidence from the structure and development of the Linear B script ${ }^{7}$

This chapter takes a new approach to the question of the undeciphered signs' possible values: rather than considering each sign individually (as has usually been the case in previous studies dedicated to identifying possible sound-values for these signs), they will be considered as a group in terms of their function within the LB script as a whole. By analysing the functions of the LB signs whose sound-values are currently known and the processes involved in developing the script - both its initial adaptation from LA and the subsequent creation of new signs - I shall address the question of what types of soundvalues are theoretically most (or least) likely to exist amongst the undeciphered signs.

Of course, that a given sound-value is judged to be probable in principle does not make it certain that a sign with such a value did exist; nor does the judgement that a given sound-value is theoretically improbable mean that such a value certainly did not exist. Rather, establishing the relative probability of different types of sound-values is intended to provide a basis for evaluating the plausibility of any given proposal for the value of an undeciphered sign - a further tool to be used alongside the other forms of evidence, such as possible spelling alternations or identifications of Greek words, which have more traditionally been used in decipherment attempts, and which will also be employed in my discussion of individual signs in Chapter 2.

\footnotetext{
${ }^{7}$ A paper based on Section 2 of this chapter is forthcoming in P.M. Steele (ed.), Understanding Relations Between Scripts: The Aegean Writing Systems (Oxford: Oxbow Books).
} 
Logically, there are three possibilities when considering the general types of soundvalue any given undeciphered sign could have, all of which have featured in previous decipherment proposals:

\section{1) its sound-value is identical to that of an LB sign whose value is known}

This is highly implausible, since there is currently no evidence to suggest that LB ever possessed more than one sign with exactly the same value; ${ }^{8}$ this possibility will therefore not be further considered in this chapter.

\section{2) its sound-value is of a type not currently known in the script}

It is, in theory, possible that an undeciphered sign could represent a Greek phonemic feature not currently known to be distinguished in the script (e.g. vowel length, or a distinction between $/ \mathrm{r} /$ and $/ 1 /$ ). This would, likewise, appear prima facie implausible, since it would be at odds with what appear to be some of the script's fundamental principles; this possibility will, however, require some further discussion at the end of this chapter (p.65).

\footnotetext{
${ }^{8}$ Although 'doublet' signs are orthographically equivalent to a single 'core' sign, phonemically they always have a more specific value: for instance, while $a$ represents a range of values (including /a/, /ha/, /ai/, and /hai/), $a_{2}$ specifically marks aspiration without specifying the quality of the vowel/diphthong (and can therefore represent /ha/ or /hai/); conversely, $a_{3}$ represents the diphthong without specifying aspiration (/ai/ or /hai/).
} 
It is also frequently claimed that some undeciphered signs may represent non-Greek, 'Minoan', phonemes. ${ }^{9}$ Given the unknown nature of the Minoan language, ${ }^{10}$ this can in practice only be discussed where signs inherited from LA may have had their original values modified to some extent for use in writing Greek - which would put these signs in exactly the same situation as the rest of the LB script. A sign used in LB only to represent a phoneme so foreign to Greek speakers as to defy reanalysis in pronunciation and/or spelling would be impossible to decipher, at least pending a decipherment of LA; but this possibility will be discussed further in Chapter 2 .

\section{3) its sound-value is of a type already known to be represented in the script, i.e. it fills a}

\section{'gap' in a series of related signs}

Attempting to assign values 'missing' from the script to undeciphered signs in order to fill such gaps is, evidently, the most potentially productive approach, and the one which has been the basis of the largest number of decipherment attempts. It is this approach on which this chapter will largely focus, by asking the question: which of these gaps are actually most likely to have been filled?

In order to answer this question, it will be necessary to examine the origins and functions of the series of signs in which these gaps exist: one of the main themes of this chapter will therefore be the processes involved in the adaptation of LB signs from LA and the creation of new signs within LB, and the purposes which the latter, in particular,

\footnotetext{
${ }^{9}$ E.g. Palaima-Sikkenga 1999:602; Melena forthcoming:4, 21, 25-6.

10 'Minoan' here refers to the language of the LA texts (which are probably all in the same language: Duhoux 1978:103-5 and 1989:92; Davis 2014:179-81), regardless of the probable existence of other nonGreek languages on Crete at this time. This may or may not, however, be the language which LA was originally created to write: see p.9, n.14.
} 
fulfilled. Of course, when discussing the former one must take into account the difficulties involved in attempting to analyse the sound-values of LA signs and the phonology of Minoan. It is often suggested that relatively radical processes of adaptation may have taken place in the creation of $\mathrm{LB}$, in particular relating to the vowel series: as all the signs in the LB $a^{-}, i^{-}$, and $u$-series have LA counterparts, but some of the $e^{-}$and many of the o-series do not, ${ }^{11}$ it is frequently assumed that LA had only three vowel series (corresponding to three vocalic phonemes in Minoan), ${ }^{12}$ so that the $e$ - or o-signs inherited from LA must have undergone a re-assignment of their sound-values. Meißner-Steele forthcoming [2017], however, argue persuasively against this view: shared correspondences of o-series signs between LB, LA, and the Cypriot Syllabary (CS), as well as the relative frequencies of these signs in LA, make it more probable that LA did have five vowel series, and that chances of attestation are responsible for the lack of known LA correspondences to some of these signs. Based on this, as well as on various other types of evidence (correspondences with CS, internal LA spelling variation, attestations of the same words in LA and LB, etc.), it seems probable that most LA signs had sound-values approximately similar to those of their LB counterparts (where these exist).$^{13}$

Of course, this does not mean that no changes of sound-values took place in the adaptation of LB, nor that the Minoan phonological system was necessarily similar to that of Mycenaean Greek. The extent to which it is valid to use evidence from LA sound-

\footnotetext{
${ }^{11}$ e-series: pe, we?; o-series: do, jo, mo, no?, qo, so, wo.

${ }^{12}$ E.g. Packard 1974:112-15; Duhoux 1989:72; Palaima-Sikkenga 1999:603-4; Beekes 2007:14. Against this view, see, e.g., Duhoux 1989:72 (offering some alternative possibilities, e.g., the existence of more than five vowels); EDG:xix-Xx (altering Beekes' previous view to suggest a five-vowel system, though with the possibility that /e/ and /o/ were not yet fully phonemic); Davis 2014:240-1 (arguing for a development of a five-vowel system out of an original three vowels).

${ }^{13}$ Steele-Meißner forthcoming. See also Packard 1974; Olivier 1975; Godart 1984; Duhoux 1989:65-71.
} 
values (even if we could be certain of these) to infer the structure of the Minoan phonological system is highly questionable: no script is ever a 'perfect' phonological representation of the language it is used to write, even if it is created specifically to write that language, and this is not even certainly the case for LA. ${ }^{14}$ Since this chapter is concerned with processes of script adaptation, it will investigate the extent to which the evidence for these processes can in principle shed light on possible LA sound-values and/or Minoan phonology, as well as how this in turn can further our understanding of the likely values of the undeciphered signs; but attempting an actual reconstruction of these LA sound-values or Minoan phonemic features is beyond its scope.

\section{1: Missing 'core' signs?}

The most obvious starting-point to look for gaps which could be filled by the undeciphered signs is within the 'basic' or 'core' syllabary: i.e. the 'grid' of (c)V signs which represent the core signs with which LB can be written in accordance with the standard spelling rules. ${ }^{15}$

\footnotetext{
${ }^{14}$ It is probable that LA and CH both developed from a common ancestral script (Owens 1996; Olivier 1997:50-1). It is often assumed that the two represent different languages as the rationale for their coexistence (e.g. Olivier 1997:50; Duhoux 1998:24-6); if this is the case (which remains uncertain), we have no way of knowing whether the language represented by this hypothetical ancestor was the same as that of $\mathrm{CH}$, LA, or neither.

${ }^{15}$ The terms 'basic' and 'core' do not imply that these signs are necessarily the default option for the spelling of any given term or in the writing of any individual scribe, only that no other signs are structurally necessary for the use of the script.
} 


\begin{tabular}{|c|c|c|c|c|}
\hline$a$ & $e$ & $i$ & $o$ & $u$ \\
\hline$d a$ & $d e$ & $d i$ & $d o$ & $d u$ \\
\hline$j a$ & $j e$ & & jo & \\
\hline$k a$ & $k e$ & $k i$ & $k o$ & $k u$ \\
\hline$m a$ & $m e$ & $m i$ & $m o$ & $m u$ \\
\hline$n a$ & $n e$ & $n i$ & $n o$ & $n u$ \\
\hline$p a$ & $p e$ & $p i$ & $p o$ & $p u$ \\
\hline$q a$ & $q e$ & $q i$ & $q o$ & \\
\hline$r a$ & $r e$ & $r i$ & $r o$ & $r u$ \\
\hline$s a$ & $s e$ & $s i$ & so & $s u$ \\
\hline$t a$ & $t e$ & $t i$ & $t o$ & $t u$ \\
\hline$w a$ & $w e$ & $w i$ & $w o$ & \\
\hline$z a$ & $z e$ & & $z o$ & \\
\hline
\end{tabular}

The LB core syllabary, as currently known

There are six gaps in this core syllabary: $j i, j u, q u, w u, z i, z u$. Three of these can be immediately discounted as plausible values: sequences of two semivowels /yi/ or /wu/ do not occur in Greek, ${ }^{16}$ and although in principle such signs could have been retained from LA, it appears more likely that these sequences would have been represented simply by $i$ or $u$ if necessary either in non-Greek words or in the plene spelling of a cluster /wRu/ or /yRi/. ${ }^{17} \mathrm{ji}$ and $w u$ would therefore not be likely to exist. $/ \mathrm{k}^{\mathrm{w}} \mathrm{u} /$ is likewise not an expected sequence in Mycenaean Greek, since the delabialisation of labiovelars adjacent to $/ \mathrm{u} /$ had already taken place, ${ }^{18}$ a sign $q u$ would have become effectively equivalent to $\mathrm{ku}$, and therefore redundant. ${ }^{19}$

\footnotetext{
${ }^{16} \mathrm{Cf}$. Meißner-Steele forthcoming [2017].

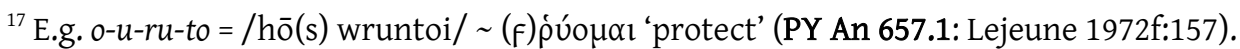

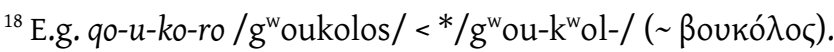

${ }^{19} q u$ could theoretically have existed in early LB (particularly if inherited from LA, as $q a$, qe, and $q i$ certainly were) if the script were invented before this delabialisation took place. The sequences $/ \mathrm{k}^{\mathrm{w}} \mathrm{u} /$, $/ \mathrm{k}^{\mathrm{wh}} \mathrm{u}$ /, and / $\mathrm{g}^{\mathrm{w}} \mathrm{u}$ / would, however, have been at best very rare (CDE lists no Greek roots originally beginning with any of these), so there would never have been a great need to write them. The only situation in which such a sign could be required by the spelling rules would be in a sequence $/ \mathrm{k}^{\mathrm{w}} \mathrm{Cu}-/$ (vel sim.), cf. qi-si-pe-e $=/ \mathrm{k}^{\mathrm{w}} \mathrm{sip}^{\mathrm{h}} \mathrm{ehe}$ / (T. Meißner, pers. comm.), but this is not likely to have been common (cf. the few examples of other $/ \mathrm{k}^{\mathrm{w}} \mathrm{C}-/$ clusters in Mycenaean: Lejeune 1972f:52).
} 
There is, however, no reason why ju should not occur, /yu/ being a perfectly possible (if not especially common) sequence, whether representing an original /y/or as a glide between $i$ and $u .^{20}$ At least two of the other $j$-series signs were inherited from LA ( $j a ~ ~$ $\mathrm{AB} 57, \mathrm{je} \sim \mathrm{AB} 46)$, and there are otherwise no newly-created signs in the $u$-series, so $j u$ would most plausibly also have been inherited. Such a sign would be expected to occur particularly frequently following $-i-.^{21}$

A sign zu could in principle be used to represent the outcome of initial */yu-/ in roots where $* / \mathrm{y}-/>/ \mathrm{dz}-/$, as well as the outcomes of $* / \mathrm{k}^{(\mathrm{h})} \mathrm{yu} /, * / \mathrm{gyu} /$, or $* / \mathrm{dyu} /{ }^{22}$ However, there are only three roots in $\zeta v-<* / y u-/$ attested in alphabetic Greek $-\zeta v \gamma^{-}(* / y(e)$ ug-/

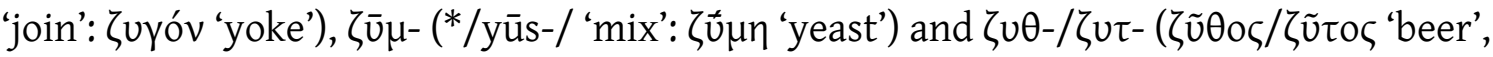
etymology unclear) - and only $\zeta \mathrm{vy}^{-}$is particularly common. ${ }^{23} * / \mathrm{k}^{(\mathrm{h})} \mathrm{yu} /,{ }^{*} / \mathrm{gyu} /$, and */dyu/ might theoretically appear either initially or medially; in practice, though, there do not appear to be any Greek roots beginning with these sequences ${ }^{24}$ and they are

\footnotetext{
${ }^{20}$ Based on spelling alternations such as jo- $\sim 0-$ and $-e-j o \sim-e-0$, it is usually assumed that the sound-change of $* / y />/ h /$ intervocalically and in some word-initial cases was either in progress or recently complete at the time of the LB tablets (see, e.g., Lejeune 1972f:169; Bartoněk 2003:139. The change of */y-/ > /dz-/ in other initial instances has already taken place: see below on $z u$ ). It is impossible to be sure whether $-j-$ in these cases represents a conservative pronunciation (i.e. the continued preservation of $/ y /$ ) or only a conservative spelling; however, the fact that the $j$-series exists and corresponds to original $/ y /$ in some cases implies that this was still phonemic at least when LB was created.

${ }^{21}$ See p.140.

${ }^{22}$ See Bartoněk 2003:142; there is no evidence for /sd/ being spelled $z$ - in Mycenaean (Meißner-Steele forthcoming [2017]), nor would this be expected according to the usual spelling rules, by which syllablefinal /-s/ is not represented. It is debated whether the value of the $z$-series is /ts, dz/ (Heubeck 1971b), /t , dz/ (Melena 2014b:53), or / $\mathrm{k}^{\mathrm{y}}, \mathrm{k}^{\mathrm{hy}}, \mathrm{g}^{\mathrm{y}}$ / (Petruševski 1979; Risch 1979); as the first option seems the most plausible, $z$ - will be transcribed as such throughout, but the actual value of $z u$ is not of great importance for the discussion here.

${ }^{23}$ LSJ; CDE; EDG, q.vv.

24 */dyu-/ or */gyu-/ would also produce $\zeta v$ - in alphabetic Greek; *// ${ }^{(\mathrm{h})}$ yu-/ would produce $\sigma v-/ \tau u-$, but $C D E$ lists no terms beginning with either sequence with these etymologies.
} 
unlikely to have been particularly common medially. ${ }^{25}$ Essentially, then, a sign zu's primary usage would be to represent the $\varnothing$-grade of the productive root $* / y(e) u g-/,{ }^{26}$ as well as potentially appearing in non-Greek words. It seems plausible that a sign zu would have been inherited from LA, since all three known $z$-series signs have LA antecedents $(z a \sim \mathrm{AB} 17, z e \sim \mathrm{AB} 74, z 0 \sim \mathrm{AB} 20)$, but not entirely certain that this sign would necessarily appear commonly enough even to be attested amongst the undeciphered signs. If it is attested, its primary usage in the root /zug-/ might give it an unexpectedly high initial frequency for a CV sign, since in this usage it would appear medially only in compounds: this could be problematic for any attempts to identify the value of undeciphered signs which are particularly frequent word-initially, which under normal circumstances would be thought to be vocalic. On the other hand, use in non-Greek words could also cause its distribution to conform more closely to that expected for a $C V$ sign, so this issue cannot be definitively settled.

If the existence of $z u$ is not entirely certain, is zi any more likely? As already discussed, Mycenaean $z$ - can originate from the palatalised sequences */ky-/, */gy-/, */dy-/, etc., and from initial */y-/; but for the sequence zi to be created from any of these would require a sequence */yi/, which, as stated above (p.10), is not one found in Greek. /zi/ would thus be expected only in non-Greek words, if at all, and whether a sign for it would exist is questionable: such a sign would be expected only if retained from LA to use in representing Minoan or other non-Greek words. ${ }^{27}$ Thus, an undeciphered sign

\footnotetext{
${ }^{25}$ Medial palatalisation is most frequently due to the addition of suffixes such as */-yos/ or */-ya/ to roots

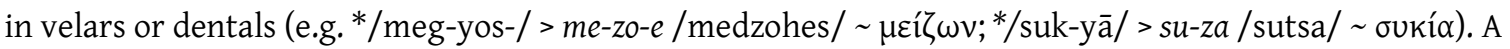
suffix */-yu-/ appears in the term */sū-yu-/ > vióc, but this is not productive in Greek.

${ }^{26}$ Attested in Mycenaean in the e-grade: $z e-u-k e-u$-si /zeugeusi/ 'for the yokers', ze-u-ke-si /zeuges(s)i/ 'for pairs' (cf. the abbreviation ZE 'pair').

${ }^{27}$ Meißner-Steele forthcoming [2017]; cf. Petruševski 1979:264.
} 
with a corresponding LA sign, found primarily in words with no clear Greek etymology (and perhaps most commonly or exclusively attested at Knossos) might be a candidate for the value zi, but it is not necessarily to be expected.

$j u$ is thus the only missing value in the basic syllabary which can fairly firmly be anticipated to underlie any of the undeciphered signs, though zu remains a reasonable possibility, and zi a more remote one. Between one and three of the undeciphered signs, therefore, may represent a value from the core syllabary; possible candidates for these values will be discussed further in Chapter 2 .

\section{2: The 'extra' signs}

A larger number of potential gaps exist within the category of 'extra' signs: those which can optionally replace one or more core signs. ${ }^{28}$ This group is usually divided into 'doublet' and 'complex' signs (with the addition of 'pseudo-complexes': Duhoux 2008:246-7) based on their orthographic function of replacing one or two core signs. However, for the purposes of this chapter I prefer a division by phonetic/phonemic function, as shown in the following table.

\footnotetext{
${ }^{28}$ The terms 'extra' and 'optional' do not necessarily imply that this cannot be the default option in the spelling of any given term or in the writing of any individual scribe, only that these signs are structurally not strictly necessary.
} 


\begin{tabular}{|c|c|c|c|c|c|}
\hline$/ \mathrm{hV} /$ & $a_{2} / \mathrm{ha} /$ & & & & \\
\hline Diphthongs & $a_{3} / \mathrm{ai} /$ & $a u$ & $\begin{array}{c}r a_{3} \\
/ \text { rai, lai/ }\end{array}$ & & \\
\hline $\begin{array}{l}\text { Aspirated (and } \\
\text { voiced?) stops }\end{array}$ & $p u_{2} / p^{h} u, b u ? /$ & & & & \\
\hline $\begin{array}{c}\text { Labialised } \\
\text { consonants }\end{array}$ & $d w e$ & $d w o$ & $n w a$ & twe & two \\
\hline $\begin{array}{l}\text { Palatalised } \\
\text { consonants }\end{array}$ & $\begin{array}{c}r a_{2} / \text { rya, lya/ } \\
>\text { /rra, lla/ }\end{array}$ & $\begin{array}{c}\mathrm{ro}_{2} / \mathrm{ryo}, \mathrm{lyo} /> \\
\text { /rro, llo/ }\end{array}$ & $\mathrm{ta}_{2} / \mathrm{tya} /$ & pte < ${ }^{*}$ pye? & \\
\hline
\end{tabular}

The LB extra signs, as currently known

It is often assumed that the existence of one or two extra signs of a certain type means that further similar signs ought to exist in order to complete the series: see, e.g., the view that '[it is] troubling...that, so far as we know, no attempt was made to balance out the Linear B script by, for example, creating or retaining signs for he and ho, ei and oi, eu and ou' (Palaima-Sikkenga 1999:604, n.19). This assumption is illustrated by decipherment proposals such as $*^{*} 2=t w a ?$ and ${ }^{*} 64=t w i ?$, based on the existing twe and two (see Melena 2014b:16-7, fig. 17.5) - but completing all of the possible series shown above would require a minimum of 30 signs, far more than the available undeciphered signs. The following discussion will therefore examine each group in turn in order to determine the relative probability of further similar signs in each category existing amongst the undeciphered signs. For a catalogue of attestations of each of the extra signs, the reader is referred to the 'Extra signs' spreadsheet, included on the enclosed CD. 


\subsubsection{Vowels}

\subsubsection{Aspirated vowels}

$a_{2} \bar{v}=/ \mathrm{ha} /$

$a_{2}$ is the only known aspirated vowel sign, aspiration being otherwise denoted only by a graphic hiatus (i.e. the writing of a sequence $/(\mathrm{C}) \mathrm{VhV} /$ as $(C) V-V$ rather than $(C) V-j V) .{ }^{29}$ It is attested at least 179 times at Ayios Vasileios, Knossos, Mycenae, Pylos, and Thebes; its value is shown by alternations with a (e.g. $\left.p a-w e-a_{2} \sim p a-w e-a\right)$ and ja (e.g. ko-ri- $a_{2}-d a-n a \sim$ ko-ri-ja-da-na). ${ }^{30}$ It has no known LA equivalent, and was therefore probably created within LB (although, as in all such cases, an absence from LA due to chances of discovery cannot be completely ruled out). $a_{2}$ 's widespread distribution suggests that this creation took place at an early stage of LB; however, its relative rarity at Knossos ${ }^{31}$ may suggest that the process of loss of $/ \mathrm{h} /$ took place earlier at this site than on the mainland. ${ }^{32}$

$a_{2}$ is found in all positions, but, contrary to the claim that it is generally attested wordinitially or at the beginning of the second element of a compound (Melena 2014b:74), in fact just $24 \%$ of its certain syllabographic occurrences are in one of these two positions; this contrasts strongly with the distributions of $a_{3}$ (p.17) and au (p.22). Although orthographically a vocalic doublet, in terms of its distribution $a_{2}$ is therefore effectively an $h$-series CV sign. Conversely, it has a remarkably high frequency of word-final

\footnotetext{
${ }^{29}$ Jiménez Delgado 2008; Meißner 2008:507-15.

${ }^{30}$ Of $a_{2}$ 's two exceptional alternations with other signs, me-nu- $a_{2} \sim m e-n u-w a$ is probably simply an example of aspiration alternating with a non-phonemic glide, parallel to alternations with ja (pace Pierini 2014:134), while $p i-a_{2}-r a \sim p i-j e-r a_{3}$ shows multiple alternations in a word of probable non-Greek origin (CDE s.v. $\left.\varphi \alpha^{\prime} \lambda \eta\right)$.

${ }^{31}$ Up to eight examples: two from the RCT, ] $a_{2}$-ta and $a_{2}-k e-t e-r e ; p a-w e-a_{2}(3 \mathrm{x}, \mathrm{H} 114)$; the theonym e-ma- $a_{2}(-o)$ (2x); and a possible example in an MN, ru- $a_{2}[$. Pylos has at least 145 examples.

${ }^{32}$ Meißner 2008:513, who suggests that the RCT may represent the stage of fluctuation between $/ \mathrm{h} / \sim \varnothing$.
} 
occurrences - 56\% - due mainly to its appearance as the ending of neuter plural s-stem nouns and adjectives (e.g. $\left.p a-w e-a_{2}, m e-u-j o-a_{2}\right)$ and in the Pylian introductory particle $o-d a-a_{2}$ (and related forms o- $a_{2}, o-d e-q a-a_{2}$ ), each of which accounts for c.25\% of total attestations and $44 \%$ of word-final attestations.

These neuter plural forms - which, unlike o-da- $a_{2}$, are found at all sites where $a_{2}$ is attested - therefore appear to be a particularly important morphological category in the use of this sign; note also that the only certain examples of $a_{2}$ from Knossos outside of the RCT are in the theonym $e-m a-a_{2}(-o)$ (which, as a religious term, is perhaps particularly likely to retain a conservative pronunciation/spelling) and the neuter plural $p a-w e-a_{2}$ (three times in H114, alongside the spelling $p a-w e-a$ in other hands), suggesting that for this scribe, at least, the spelling of this term with $-a_{2}$ may have been a particularly firmly established one. Moreover, neuter plurals in -a occur almost exclusively at Knossos: of those found in both $-a$ and $-a_{2}, p a-w e-a$, qe-te- $a$, and te-tu-ko-wo- $a$ appear only at Knossos (qe-te- $a$ and te-tu-ko-wo- $a$ once each, ${ }^{33}$ pa-we-a c.30 times) ${ }^{34}$ However, although Pylos has numerous neuter plural terms in $-a_{2}{ }^{35}$ and other mainland

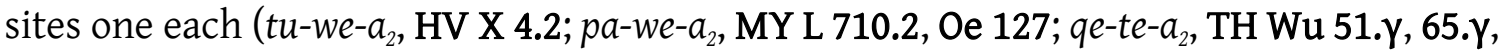
96. $)$, the only mainland examples in - $a$ are tu-we- (PY Un 267.3, H1), we-]je-ke-a (PY Wa 1148.2, H26: probably $=w e-j e-k e-a_{2}$, an obscure neuter plural adjective) and ]ko-wo-a (IK X 1, probably a perfect participle ending: Shelmerdine 2012:75-6); spelling these terms with $-a_{2}$ is thus overwhelmingly preferred (and not the 'sporadic procedure' claimed by Melena 2014b:77). This preference is all the more striking given that this spelling is,

\footnotetext{
${ }^{33} \mathrm{Fp}$ 363.1, L 871.b (NB that this is ascribed to H114?); cf. a-ra-ru-wo-a (never spelt with $-a_{2}$ ) in the Ra-series. ${ }^{34}$ Lc-, Ld-, and L-series, passim.

${ }^{35} a-k e-a_{2}, k e-r e-a_{2}$, no-pe-re- $a_{2}$, te-tu-ko-wo- $a_{2}$, we-je-ke- $a_{2}$, me-u-jo- $a_{2}$, me-zo- $a_{2}, q e-t e-a_{2}$; perhaps also ru-de- $a_{2}$, ]-we-e- $a_{2}$.
} 
arguably, redundant, since the graphic hiatus of $p a-w e-a$ etc. marking the presence of /h/ elsewhere is perfectly acceptable. It is, therefore, worth asking whether, in addition to more obvious potentially contributing factors (including greater accuracy of phonological representation and disambiguation of similarly-spelt terms, particularly those with initial /a-/ vs. /ha-/), the distinctive representation of $s$-stem neuter plurals a relatively common category in documents which frequently list and describe groups of objects - may have been an important factor in the creation of $a_{2}$.

\subsubsection{Diphthongs}

\section{i-diphthongs}

$a_{3} \stackrel{2 !}{T}=/ \mathrm{ai} /$

This sign (attested at least 103 times at Knossos, Midea, Mycenae, Pylos, Thebes, perhaps Khania, and on ISJs) is almost exclusively found word-initially, the only certain exceptions being two appearances at the beginning of the second element of a compound or juxtaposition ( $a u-t o-a_{3}-t a / a u-a_{3}$-ta /Auto-aitās $/ ?{ }^{36}$ de-we-ro- $a_{3}-k o-r a-i-j a$ /Dewero-aigolaia/). ${ }^{37}$ Since noting an $i$-diphthong is optional in LB, $a_{3}$ alternates with $a$ (or, when another vowel follows, $a-j-) .{ }^{38}$ There are no examples of $* a_{3}-j V-$ : the consistent spelling of a-ja-me-no/-na (/ayaimenos, -na/ or /aiāmenos, -na/?) with a-ja- at both

\footnotetext{
${ }^{36}$ The latter (KN C 1582.b) may well be a mistake for the former (KN Ch 972).

${ }^{37}$ Possible examples of $a_{3}$ in non-initial position: ]- $a_{3}\left[\right.$ (KN X 9698); ]-șe- $a_{3}\left[\right.$ (KN X 9806); me[ ]jo- $a_{3}$-se-wa[ (PY An 615.13); ] $a_{3}-a_{3}-t a$ (KR Z 1). The first two are very fragmentary; the next could equally be read $m e\left[\right.$ ]jo $a_{3}-$ se-wa[ (PoN IV; the previous reading is that of PTT); and the last, while a plausible reading, is perhaps a special case given the sign's repetition.

${ }^{38}$ The spelling $a-i-$ probably represents /ahi-/, though this is not entirely certain as it appears only in the PN $a-i-q e-u$ (gen. $a-i-q e-w o$, dat. $a-i-q e-w e)$ : Pylos, $\mathrm{H} 1$ and H41's E-series. Note that these two scribes regularly use $a_{3}$ (Lejeune 1972d:82).
} 
Knossos and Pylos, including by one scribe (PY H2) who has used $a_{3}$ five times elsewhere, suggests that a spelling $* a_{3}-j V$ - was perhaps felt to be redundant.

$a_{3}$ has no generally accepted LA correspondence; however, Palaima 2003 argues that the sign incised on the handle of a cauldron from Mycenae's Grave Circle A is an Imageremoved for copyright example of AB43. MY Zf 2 (right), ${ }^{39}$ as this is classed by Younger (LA Other), reasons does appear morphologically close to $a_{3}$, with the only major difference being the downwards direction of the stroke at the left; but Palaima rightly points out that this is a much greater obstacle to its alternative comparison to AB28 $Щ$ (= L100: Grumach 1962), which is equated with LB $i$ ₹ by GORILA ${ }^{5}$ :xxii, but could instead be

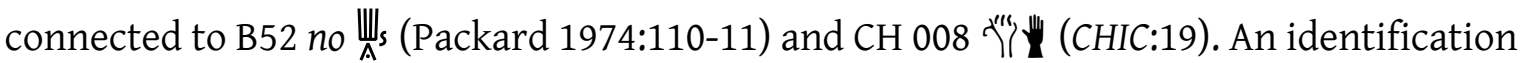
of this sign as AB28 seems to me almost impossible due to the triangular base; (A)B52 is a better possibility, since uncanonical forms of no, with the 'thumb' placed in different positions, are not unknown on non-administrative inscriptions, ${ }^{40}$ but still morphologically less good than (A)B43. ${ }^{41}$

However, this sign is still highly insecure as an example of AB43. As Palaima himself points out, the cauldron's LH IB date is in theory as compatible with this being an early example of $\mathrm{LB} a_{3}$ as a late LA AB43. ${ }^{42}$ Additionally, the sign's occurrence in isolation on a

\footnotetext{
${ }^{39}$ Image: author (NAM).

${ }^{40}$ See Judson 2013:79 on TH Z 857 and 858.

${ }^{41}$ The suggestion of Younger (LA) (s.v. 9. Language) that this sign could also be related to A306 (L83) should be rejected on palaeographic grounds. The possible evidence presented by Packard 1974:108-9 and Younger (LA) for A306 having a value AI or $\mathrm{A}_{2}$ is also highly insecure, being based on a series of possible LA alternations involving very short and/or incomplete terms; Packard's specific suggestion of AI relies on two similarly insecure LA/LB alternations.

${ }^{42}$ The date of the creation of LB is of course unknown, but the earliest widely suggested date is MM III/LM IA (Palaima 1988a:273-5); LM IA $\approx$ LH I. The main period of LA's attestation is MM III-LM IB (Del FreoZurbach 2011:75, 84-5).
} 
vessel means caution is needed in identifying it as belonging to a script at all, since although isolated marks on pottery sometimes seem to be related to signs of contemporary writing systems, ${ }^{43}$ fairly simple marks can equally appear similar to signs of these writing systems by chance.

If, as still therefore seems most probable, $a_{3}$ was created within LB, what may have been the reason(s) for this? Clearly, a sign attested only word-initially cannot have a morphological motivation, as was argued for $a_{2}$. Probably influence from the pre-existing $a u(p .22)$ may have encouraged the creation of a corresponding means of representing initial /ai-/ accurately, concisely, and unambiguously, thus enabling (in principle) the clear distinction of terms beginning with /ai-/, /a-/, and /ahi-/.

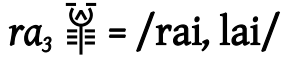

This sign, although found as the 'saffron' ideogram at Knossos (CROC), is found as a syllabogram only at Pylos; ${ }^{44}$ its lack of ideographic examples at the latter site is presumably due to chances of attestation, since an acrophonic derivation from a word meaning 'saffron' seems the most likely process of assigning this sign a sound-value. ${ }^{45}$ It is uncertain whether the lack of a corresponding LA ideogram is similarly due to chance. ${ }^{46}$ However, the lack of syllabographic examples at Knossos (despite the ideogram

\footnotetext{
${ }^{43}$ Hirschfeld 1990 [unpublished]:53-65.

${ }^{44}$ Lejeune $1972 \mathrm{~d}$ refers to a syllabographic example on $\mathrm{KN} \mathrm{X} \mathrm{7739,} \mathrm{but} \mathrm{this} \mathrm{is} \mathrm{now,} \mathrm{much} \mathrm{more} \mathrm{plausibly,}$ read *18: see p.72.

${ }^{45}$ Presumably this would have been a non-Greek word (as kpókoৎ also is in origin), though no such term beginning with /rai-/ or /lai-/ is known (T. Meißner, pers. comm).

${ }^{46} \mathrm{CH}$ 023/159bis $\mathrm{M} /$ is a possible correspondence (CHIC:19; Karnava 2000 [unpublished]:240). However, there is no evidence that this sign represents saffron; Younger (CH) (s.v. Notes on the Signs: 023$)$ connects it with AB122 = OLIV $M$, which seems a better formal correspondence.
} 
being attested at least 39 times) or any other site is probably significant, indicating that the assignment of this sound-value took place only at Pylos.

The Pylian syllabogram is reasonably well-attested, with at least 20 examples; it is found in medial and final position, the nine final examples all being in a-stem nominative plural nouns (f.: di-pte-ra $a_{3}, p i-j e-r a_{3} ;$ m.: o-ka-ra $_{3}$ and adjectives (f. ethnics: $k u-t e-r a_{3}$, $\left.z e-p u_{2}-r a_{3}\right){ }^{47} a$-stem nominative plurals are thus a significant component of the corpus, representing $45 \%$ of certain attestations and $56 \%$ of lexemes in which this sign appears. Strikingly, there are no examples of $r a_{3}$ being used in an $a$-stem dative singular /-ăi/, which is otherwise homographic with the nominative plural /-ai/; the fact that three scribes who have used $\mathrm{ra}_{3}$ also have probable examples of dative plurals in /-rai/ or /-lai/ written -ra suggests that the restriction of this sign to one of these two case-forms may have been deliberate ${ }^{48}$ and that the resulting ability to distinguish the nominative plural from not just the dative singular but also the rest of the $a$-stem paradigm (most of whose cases are likewise spelt - $a$ ) may have been at least partially responsible for motivating the creation of $r a_{3}$.

It is noticeable that this convention, like the preference for the spelling $-a_{2}$ in representing neuter plurals, results in the more distinctive representation of a plural

\footnotetext{
${ }^{47} \mathrm{There}$ are no examples of ${ }^{*} \mathrm{ra}_{3}-\mathrm{jV}$-, and though the sample size is small, this is concordant with the similar case of $a_{3}$ (pp.17-18). Moreover, the name of the Further Province, /Perāigalaion/, is spelt as both pe-ra $a_{3}-k o-$ $r a-i-j a(\mathrm{Ng}$ 332.1, Wa 114.2) and pe-ra-ko-ra-i-ja (Pa 398.a), and the corresponding ethnic adjective as pe-ra-ako-ra-i-jo (On 300.8:/Perā-aigalaioi/, nom.pl.): although these differ in their notation of /-r(ā)ai-/, /laiV/ is consistently spelled $r a-i-j V$.

${ }^{48}$ au-to-*34-ta-ra is a recipient on Fn 187.10 (H2); ke-sa-da-ra is the recipient of rations on $\mathrm{Fg} 368$ (H21) and 828 (H1), and probably also appears on An 435.2 (H1: ke [-] assigning a workforce (Nakassis 2012:279-81). The syntax of this last example guarantees the dative case; although nominatives of rubric cannot be ruled out in the cases of the recipients, au-to-*34-ta-ra, at least, is attested alongside several unambiguous datives.
} 
form (when, in this case, there is no particular orthographic or linguistic reason why $-r a_{3}$ could not instead be restricted to representing the dative singular). A further parallel for this kind of orthographic convention may be found in the more general representation of $i$-diphthongs, which are governed by a relatively complex set of conventions according to which the orthography varies depending on word-position: while a medial $i$-diphthong may be written either $-V-(j-)$ or $-V-i-(j-)$ (with some variation by site: the plene spelling is more common at Knossos than Pylos), the latter spelling is restricted word-finally (and probably initially: p.17, n.38) to representing hiatus, /Vhi/, as shown by the spelling of case-forms such as the $a$ - and $o$-stem dative singular and plural $(-a=$ /-ằi/, -o = /-ŏ̀i/; - $a-i=/-a i(h) i /$ or /-ā(h)i/, $-o-i=/-o i(h) i /) .{ }^{49}$ Clearly, the potential for ambiguity to cause serious problems of interpretation is far greater in the latter case. In terms of orthographic convention, there is no reason why final /-Vi/ and /-V(i)hi/ could not both have been spelt $-V-i$; but this would have led to identical representations of dative singulars, nominative plurals, and dative plurals, while nominative singulars would still have been identical to accusative and genitive singulars. Reserving the orthography $-V-i$ for /-V(i)hi/ thus enables dative plurals, at least, to be distinguished.

All of these conventions point to a particular importance attached to the distinction of number over case. In the context of documents whose syntax is relatively simple and whose main concern is accounting, this makes sense: ambiguities of case are likely to be resolvable from context and hence relatively unproblematic (witness the frequency of nominatives of rubric), while ambiguities of number could lead to numerical mistakes

\footnotetext{
${ }^{49}$ Lejeune 1972d:83-4. Bartoněk 2003:165, 188 also includes /-ais/? and /-ois/? as possible forms of these dative plurals but, as discussed by Bartoněk (p.167), this is not a plausible suggestion.
} 
and miscalculations..$^{50}$ This is not to say that case was unimportant: clearly, if case could be easily distinguished in accordance with the script structure and orthography, it would be; but if a choice had to be made under the constraints of the existing script structure whether to represent a greater number of case distinctions or number distinctions, it seems that the latter would be preferred.

\section{$\underline{\text { u-diphthongs }}$}

au

This is the only currently-known member of this category, attested at least 46 times at Knossos, Mycenae, Pylos, and Thebes, with a further 67+ attestations in its function as the ideogram sus. As expected for a vocalic sign, au has a similar distribution to $a_{3}$, being found only word-initially. ${ }^{51}$

In principle, au should alternate with $a-u$-, since (unlike $i$-diphthongs) $u$-diphthongs are standardly spelt in full; however, there appear to be no actual examples of such an alternation. ${ }^{52}$ In fact, there are only two certain instances of initial $a-u-, a-u-q e$ (KN Sd 4402.a) and a-u-ta-na (KN Xd 7649), of which the first is most plausibly a scribal error for o-u-qe; it is tempting to interpret $a-u-t a-n a$ as /ahu-/, just as initial $a-i-$ probably

\footnotetext{
${ }^{50}$ Such a development would, however, seem unlikely to have taken place in the context of an administration which also produced longer documents with more complex syntax. This therefore reinforces the view that LB's primary use was to write on clay tablets, rather than also being used for longer-term records on other media; cf. the conclusion of Bennet 2001:27-30 that such longer-term records appear to be neither required by the Mycenaean administrative system nor suggested by the evidence of the tablets themselves.

${ }^{51}$ The few examples which are not certainly word-initial are all due to possible breaks: ]au-ri-jo (KN Dv 1103.B), ]au[ (KN X 6008), and ]ạu [ (KN Dv 8637).

${ }^{52}$ There are some instances of au over erased [[a]] (PY Ta 711.2, An 1281.4?; MY Au 657.2), but it is not clear whether the scribes' 'mistake' in these cases was to start writing $a-u$ - or only $a$-.
} 
represents /ahi-/, but the term is obscure (Lejeune 1972e:185). Although there are more examples of word-initial $a-w V-$, where these are interpretable this always represents /awV-/ (e.g. a-we-ke-se-u = /Awekseus/) not /au(w)V-/ (whereas $a-j V-c a n=/ a i(y) V-/)$.

$\mathrm{au} / \mathrm{sus}$ is related to AB85, which is similarly used both syllabographically and ideographically in LA; a possible $\mathrm{CH}$ correspondence, $017 \odot$, is found only as a syllabogram (CHIC:19). As there are no more than four examples of syllabographic AB85 (which occurs initially and medially) ${ }^{53}$ and five of $\mathrm{CH} 017$ (in all positions: CHIC:329), there is little evidence for their possible value (the numbers are too low to say whether these signs' distributions are significant). With no strong evidence to the contrary, it seems most plausible that AB85, at least, had a value similar in some way to /a/ (whether or not it was actually a diphthong) which led to its use for / au/ in LB, but this cannot be proven. ${ }^{54}$ The apparent lack of alternative spellings for initial /au-/ may be explained by the fact that au was inherited from LA: there would therefore never have been a period in which any other spelling of /au-/ was needed, whereas the spelling $a-(j-)$ for /ai-/ presumably originates from the period prior to the (probable) LB invention of $a_{3}$.

\footnotetext{
${ }^{53}$ Initial: IO Za 3, KH 6.7, KO(?) Zf 2? (GORILA); medial: ZA Zg 35 (Kopaka 1989).

${ }^{54}$ Some support for this could be found in the possible alternation AB85-59-45 (KO(?) Zf 2) AB08-59-45 (CR(?) Zf 1), AB08 very probably having the value /a/ in LA as in LB (Tsipopoulou et al. 1982:69), but as the former text lacks word-dividers, this sequence cannot be certainly identified as a separate word. Younger (LA Other) (s.v. $\mathrm{PH}($ ?) 31 ) suggests that the ligatures AB37+85 and AB37+85+27, which if read with LB values would be SI-AU and SI-AU-RE, could be the equivalent of LB si-a $a_{2}-r$, acting as a phonetic complement of ovIs, which the former follows on $\mathrm{PH}(?) 31$ b.4. However, the latter is clearly acting as an ideogram itself on $\mathrm{PH}(?) 31$ a.3; the formatting of both is that of a ligature, not a phonetic sequence; the comparison si- $a_{2}-r o$ $S I-A U-R E$ is phonetically difficult; and moreover si-a $a_{2}-r o$ and $\sigma i ́ \alpha \lambda$ os refer specifically to pigs.
} 


\subsubsection{Summary of vowels}

Theoretically possible options for further vowel signs include aspirated versions of all four other vowels and diphthongs of $e$ and $o$, as well as further signs of the type CVi, or indeed $\mathrm{CVu}$. Even more specific signs are in theory also possible, e.g. a sign specifying the value / hai/ ${ }^{55}$ However, the benefit of being able to distinguish unambiguously between /ha/, /ai/, and / hai/ would be unlikely to outweigh the disadvantage of adding another sign to the syllabary; it is, moreover highly doubtful that a single sign could specify two phonemic features (e.g. [+aspiration] and [+-i]) in this way.

\section{/h-/}

Since $a_{2}$ has no known LA correspondence, there is no reason to think that signs for other aspirated vowels would have been inherited ( $a$ being the most frequent vowel in LA: Davis 2014:262). Would these have been created in LB at the same time as $a_{2}$ ? The answer seems almost certainly to be no, since not only is /a/ also the most frequent vowel in Greek - and /ha/ correspondingly the most frequent aspirated vowel - but also because of the morphological role which $a_{2}$ seems to have played. Signs for /he/ and /ho/ would be of use word-initially (in enabling a distinction between similar lexemes) far less often than $a_{2}$; in medial or final position, they would likewise be redundant in strict orthographic terms as a representation of hiatus, without obviously offering any particular benefit in morphological identifications (although both sequences appear in Mycenaean noun paradigms, the spelling $-e-0$, for instance, is already fairly clearly an $s-$ stem genitive ending, and a sign for /ho/ would do nothing to resolve its ambiguity between singular /-ehos/ and plural /-ehōn/). Signs for /hi/ or/ hu/ would not suffer from such redundancy, and would in fact be arguably more useful than $a_{2}$ in enabling the

\footnotetext{
${ }^{55}$ See pp.97-8.
} 
unambiguous distinction of a diphthong from a hiatus, with / hi/ being of particular use in distinguishing dative case-forms; but we have already seen the orthographic convention that final $-V i$ diphthongs are spelt $-V$, so that final $-V-i$ can only represent hiatus (p.21). If a sign for / hi/ had originally existed, it seems inconceivable that such a convention would have developed; conversely, since this system appears to have functioned effectively enough, there is not likely to have been any particularly strong motivation to invent such a sign at a later stage. Given that the (morphologically) relatively strongly-motivated sign /hi/ therefore probably did not exist, there is no strong reason to suppose that $/ \mathrm{hu} /$, with no such motivation, did either. ${ }^{56}$

\section{/Vi/, /Vu/}

It is in theory possible that LA signs similar to au (whether representing diphthongs or, e.g., different vowel qualities or lengths) could have produced LB eu and/or ou, and/or that further $V i, V u$ signs could have been created on the model of $a u$ and $a_{3}$. Since the orthographic representation of diphthongs of $e$ and $o$ is in all other respects the same as those of $a$, if signs for the former existed we would expect to observe the same distribution pattern: word-initial /ei-/ and /oi-/ might be represented optionally by $e^{-(j-)}$ and $o_{-}(j)-$, but $e^{-i-}$ and $o_{-i-}$ should appear only rarely and should (probably) represent /ehi/ and /ohi/. /eu/ and /ou/ should likewise be represented only rarely, if at all, by $e-u$ - and $o-u$ - word-initially. The latter is certainly not the case, as both are frequently found, ${ }^{57}$ for instance in compounds in /eu-/ or /euru-/ (e-u-da-mo=

\footnotetext{
${ }^{56}$ Note also that in later (non-psilotic) Greek dialects initial $\dot{v}$ is always aspirated (see Lejeune 1972f:280-1); if this was also the case in Mycenaean (which cannot be determined for certain), the lack of contrast between initial /u-/ and /hu-/ could also have decreased the possible motivation to create a sign hu (T. Meißner, pers. comm.).

${ }^{57}$ This certainly applies at Knossos and Pylos. Mycenae has one probable example of each diphthong: e-upo-ro-qe = /Euporos, Eup ${ }^{\mathrm{h}}$ oros, Eupōlos/?; o-u-ko = /ouk $\mathrm{h}_{\mathrm{h}}^{\mathrm{os}} /$ 'vow'? (debated, but the alternative interpretation /owikos/ 'of sheep' is orthographically difficult). At Thebes, e-u-te-re-u may = /Eutreus/
} 
/Eudāmos/) and as the negative particle /ou-/ (o-u-di-do-si = /ou-dido(n)si/). eu and ou signs are therefore not likely to have existed in LB either through inheritance or later invention.

There are, however, no certain examples of initial $e^{-i-}$ or $0-i-{ }^{58}$ nor of $e_{-j-},{ }^{59}$ while $0-j-$ appears only in the terms o-ja-de (KN Fs 9.B: probably a TN) and o-je-ke-te-to (TH Fq 130.1); ${ }^{60}$ a survey of DMic also shows relatively few possible examples of $e^{-}=/ \mathrm{ei}^{-} / \mathrm{and} \mathrm{o}^{-}=$ /oi- /. ${ }^{61}$ This means that, although it remains uncertain whether there were in fact signs for /ei/ and/or /oi/, there are at least spaces available in which such signs could exist. If, therefore, any undeciphered sign is found to have a distribution suggesting a vocalic value, then one of these $i$-diphthongs will be the most plausible value. Note that, although we would normally look for a high proportion of occurrences preceding $-j$ - as a marker of a sign in $-i$, the lack of any such examples of $a_{3}(p .17)$ and $r a_{3}(p .20$, n.47) suggest that this might well not be the case. Given the lack of corresponding $u-$ diphthongs, these potential $i$-diphthong signs would have to either have been created in LB on the basis of $a_{3}$, or based on LA signs with values similar to /ei/ or /oi/ (although, like AB85 [p.23], these need not necessarily have represented diphthongs in LA).

$\left(T F C^{1}: 263\right) ; 0-u-k o-w e-i$, an obscure MN, is also spelt o-ko-we-i $\left(T F C^{1}: 394\right)$, and it seems easier to explain this as a mistake for, or an unusual spelling of, /ou/ than a representation of /ohu/. At Khania, e-u-ko-ro (Ar 4.1) may $=\mathrm{KN} e$-u-ko-ro (/Eukoros, Euk ${ }^{\mathrm{h}}$ olos, Euklos/?; E. Hallager 2011:420), but this could also be read e-da(e.g. Godart-Tzedakis 1995:25). ${ }^{58}$ The only possible instance of the former is ]e-i-ja-si (KN B 804.3; obscure). ${ }^{58}$ The only possible instance of the former is ]e-i-ja-si (KN B 804.3; obscure).

${ }^{59} \mathrm{DMic}$ gives one possible example, $e[?] j \mathrm{jo}$ (KN Db 5310.B) but this is now read e-qa-jo or e-ri-jo (KT ${ }^{5}$, COMIK).

${ }^{60}$ For discussion of this term, see $T F C^{1}: 195-6$; Chadwick 1996-1997:293-6.

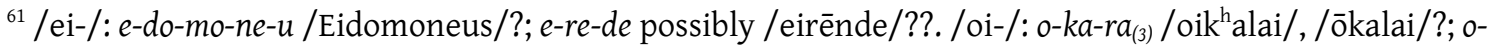

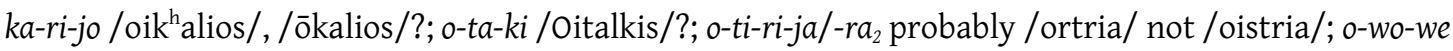
/oiwōwēs/. 
$/ \mathrm{CVi} /, / \mathrm{CVu} /($ where $\mathrm{V}=a, e, o)$

In principle, the existence of $\mathrm{ra}_{3}$ means that further signs of this type could have been invented - the most obvious possibility is $\mathrm{ro}_{3}$, for use in representing o-stem plurals, but other signs for /Cai/ or / Coi/ could be of similar use in roots ending with other consonants (/Cei/ signs would be less likely as these would enable the distinction only of $3^{\text {rd }}$ declension dative singulars rather than any plural forms; / $\mathrm{CVu} /$ signs would be of little morphological use and are therefore judged to be improbable). In practice, however, further such signs are unlikely due to the lack of undeciphered signs with a suitable distribution: there are none attested exclusively at Pylos, so that further Pylian inventions of similar signs do not appear to have taken place. ${ }^{62}$ While an independent invention of a similar sign at Knossos - the only site at which any undeciphered signs $\left({ }^{*} 49\right.$ [p.104], and perhaps * 18 [pp.71-3]) are uniquely attested - cannot be entirely ruled out, the chances do not seem high; nor is there any evidence to suggest that the Pylian invention of $\mathrm{ra}_{3}$ was based on an existing similar sign in more general use. The probability of further CVi signs existing is therefore judged to be low.

\subsubsection{Aspirated (and voiced?) consonants}

$p u_{2}+\$+=/ p^{h} u, b u ? /$

This is the only sign which indicates a specific phonetic feature of a stop (aspiration and perhaps voicing), as opposed to a feature of a vowel or a consonant cluster. It is found at least 52 times at Knossos, Pylos, Mycenae, Thebes, and on the ISJ EL Z 1, and has a clear LA antecedent, AB29 挂. ${ }^{63} \mathrm{pu}_{2}$ 's status as a doublet of $p u$ is shown by alternations such as

\footnotetext{
${ }^{62}$ The absence of $\mathrm{ro}_{3}$ from this site could perhaps be due to the lack of a suitable ideogram corresponding to a word beginning /roi-/ or /loi-/.

${ }^{63}$ The suggestion of Docs ${ }^{2}: 33$, fig.6 that this also corresponds to $\mathrm{CH} 058 \mathbb{8}$ seems palaeographically implausible.
} 
$a-p u_{2}-k a(-n e) \sim a-p u-k a$ (the same ethnic adjective spelt differently by two scribes in the PY A-series) ${ }^{64}$ or $p u_{2}-k e \sim p u-k e(-o)$ (the same MN spelt differently by two scribes in the MY Ge-series).$^{65}$ The value / $\mathrm{p}^{\mathrm{h}} \mathrm{u}$ / is plausibly established from identifications of terms

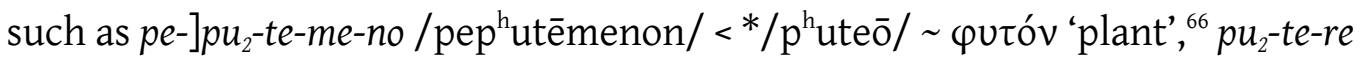
/phutēres/ 'planters', and su-ko-pun-te-e, probably /sukoph utehe(i)/ 'fig-planter' (appellative or MN), ${ }^{67}$ plus various other less secure (but still plausible) interpretations of PNs or TNs such as $p u_{2}-t i-j a=/ \mathrm{P}^{\mathrm{h}} u \mathrm{t}^{\mathrm{h}} \mathrm{iās} /$ ? (MN). By contrast, the only widely-accepted interpretation of a term in which $p u_{2}=/ b u /$ is da-pu $u_{2}$-ri-to-jo $=/$ daburint ${ }^{\mathrm{h}}$ oio/ "of the labyrinth'; it is therefore worth examining this term in some detail. ${ }^{68}$

The interpretation of $d a-p u_{2}-r i-t o-j o$ as 'labyrinth' is contextually plausible given the religious context of its attestations: $\mathrm{KN} \mathrm{Gg(1)} 702$ lists offerings of honey to 'all the gods' and the 'Potnia da-pu - -ri-to-jo', and $\mathrm{KN} \mathrm{Oa} 745.2$ an offering of * $166+W E$ to the same goddess (da-pu $u_{2}$-rị[-to-jo ]po-ti-ni-jạ).$^{69}$ Linguistically, however, the comparison of da-pu ${ }_{2}$-ri-to-jo to $\lambda \alpha \beta u ́ p ı v \theta o \varsigma$ requires an explanation of the nature of the first and second consonants. The first appears to be an instance of the /d/ /1/ alternation seen in some

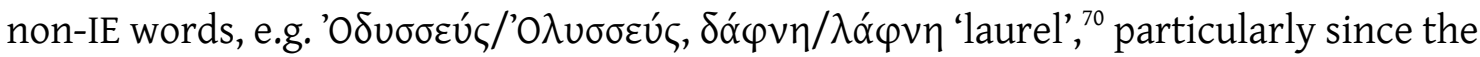
suffix /-nt ${ }^{\mathrm{h}}$ os / already points to a non-Greek origin. ${ }^{71}$ This leaves several key issues

\footnotetext{
${ }^{64} a-p u_{2}-k a(-n e)$ : An 656.13.20, 657.13 (H1); a-pu-ka: Aq 218.15 (H21), cf. KN Uf(1) 111.a, Xd <331>.

${ }^{65} \mathrm{pu}_{2}$-ke(-o): Ge 602.2, 605.2B, 608.4B (H57); pu-ke: Ge 603.2, 604.4 (H58a).

${ }^{66}$ The restoration and interpretation seem probable in the context of a landholding tablet (PY Er 880).

${ }^{67}$ Aravantinos et al. 2008:28; Thompson 2014:174-5.

${ }^{68}$ The only argument I am aware of that $p u_{2}$ represents only /bu/ is that of Witczak 1993a, which is based chiefly on a proposed acrophonic value of ${ }^{*} 22=p i_{2}=/ \mathrm{bi} /$ derived from Pre-Greek / bisōn/ 'buffalo', plus etymological interpretations of various PNs and TNs (see also p.91, n.248). It hardly needs stating that this is not a reliable basis for such an argument.

${ }^{69} \mathrm{Cf}$. KN Xd 140, da-pu-ri-tọ [ (context unclear).

${ }^{70} E D G$ :xxviii.

${ }^{71} E D G$ :xxxiii-iv.
} 


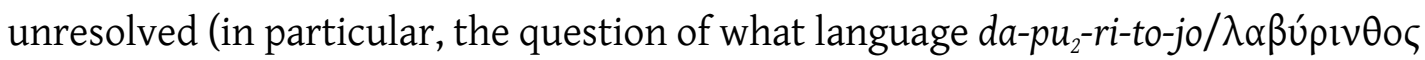
originated from, ${ }^{72}$ and why there are no synchronic examples of this alternation in this term) ${ }^{73}$ and is perhaps, therefore, somewhat less secure than it is often presented as being. However, it would also be a rather large coincidence to have a term phonetically similar but etymologically unrelated to $\lambda \alpha \beta u ́ p ı v \theta o \varsigma$ attested in a religious context at Knossos. In any case, for the sake of considering the status of $p u_{2}$ it is necessary to consider the implications of $d a-p u_{2}$-ri-to-jo in fact being 'labyrinth'.

In order to resolve the difficulty concerning the LB spelling - $p u_{2}$ - compared to classical $-\beta-$, it is generally assumed that $d a-p u_{2}-r i-t o-j o=/$ daburinthoio/, demonstrating that $p u_{2}$ can represent $/ \mathrm{bu} /$ as well as $/ \mathrm{p}^{\mathrm{h}} \mathrm{u} /$; however, this requires an explanation of how a single doublet could specify two different phonetic features, [+aspiration] and [+voicing], since this is unparalleled in LB. Melena 1987b:227-30 suggests that, when LB was adapted from LA, the IE voiced aspirates had not yet become devoiced; hence originally pu was unmarked for voicing (i.e. /pu/, /bu/, or $/ \mathrm{b}^{\mathrm{h}} \mathrm{u} /$ ) and $p u_{2}$ was marked as voiced (i.e. /bu/ or $\left./ b^{h} u /\right)$. Thus, when devoicing took place, $p u_{2}$ would have remained in use in conservative spellings of terms containing $* / b^{h} u />/ p^{h} u /$, bringing about the synchronically odd situation of a single doublet representing two different phonetic features.

\footnotetext{
${ }^{72} \lambda \alpha \beta u ́ p i v \theta$ o has no good etymology from any language, despite attempts to connect it to, e.g., Greek $\lambda \tilde{\alpha} \alpha \varsigma$ 'stone', $\theta \alpha \dot{\alpha} \tau \tau \omega$ 'bury', or $\lambda \alpha u ́ p \alpha$ 'narrow passage'; the supposed Lydian word $\lambda \alpha \beta_{\beta} \rho v \varsigma$ 'axe' (Plutarch Moralia 302a); Carian Labraunda (site of a temple of Zeus Labra(u)ndos); a reconstructed South Anatolian verb * JaBar- 'rule'; and even Hebrew debîr 'inner sanctum of the Temple' (CDE, EDG q.v.; Aspesi 1996a; Gallavotti 1957b; Yakubovich 2002; Valério 2007; but cf. Valério 2015:329-32). Possible connections between da-pu $u_{2}-r i-$ to, the PNs $d a-p u_{2}-r a-z o$ and $d u-p u_{2}-r a-z o$ (Lejeune 1972b:95-6), and/or LA (-)AB51-29-27/(-)DU-PU $-R E$ (Aspesi 1996b; Valério 2007 and 2015) are of little help in this regard. ${ }^{73}$ The suggestion that /d-/ may have been the original form, with classical /1-/ (first attested in Herodotus) being the result of transmission via an Anatolian language lacking initial /d-/ (Valério 2015:332, n.6) is evidently highly speculative.
} 
However, the devoicing of the voiced dentals clearly occurred before LB's creation, since the $t$-series, and not the $d$-series, is consistently used to represent aspirated dentals: ${ }^{74}$ e.g.

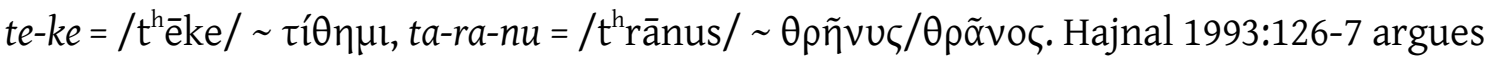
that this devoicing could have taken place as two separate developments, with $* / \mathrm{d}^{\mathrm{h}} />$ $/ \mathrm{t}^{\mathrm{h}} /$ and $* / \mathrm{g}^{\mathrm{h}} />/ \mathrm{k}^{\mathrm{h}} /$ (and presumably $* / \mathrm{g}^{\mathrm{wh}} />/ \mathrm{k}^{\mathrm{wh}} /$ ) in the 'urgriechisch' phase but $* / \mathrm{b}^{\mathrm{h}} /$ being preserved into the Mycenaean period; but he offers no explanation as to why an identical sound change should have affected different consonants at such different times. Given the identical outcome of the voiced aspirates in all dialects (in contrast to the varying post-Mycenaean developments of the labiovelars) ${ }^{75}$ it is clear that this devoicing must have taken place as a single process affecting all the voiced aspirates simultaneously.

Davis 2014:214-20 suggests an alternative explanation: that AB29 stood for a bilabial fricative with voiced and unvoiced allophones $([\beta],[\phi])$, hence $p u_{2}$ 's ability to represent both $/ \mathrm{p}^{\mathrm{h}} /($ the closest Greek phoneme to $[\phi])$ and $/ \mathrm{b} /$ (the closest to $\left.[\beta]\right)$. Although the suggestion of a value (similar to) / $\beta$ / for AB29 may be an attractive one (see further pp.31-2), this is still highly uncertain, and there are methodological difficulties with a theory that depends on identifying not just an unknown language's phonemic inventory but also its patterns of allophonic variation.

There is, moreover, no particularly good reason for LB to have had a specific means of representing /b/, a phoneme which is likely to have been at best extremely rare in

\footnotetext{
${ }^{74}$ Lejeune 1972f:30-1.

${ }^{75}$ Thompson 2005:112-3.
} 
Mycenaean Greek; ${ }^{76}$ arguments for $p u_{2}=/ b u /$ which rely on the supposed need for structural balance within the writing system, citing the existence of the $d$-series, ${ }^{77}$ ignore the fact that the latter is clearly the unusual feature. ${ }^{78}$ In the absence of any convincing explanation for how $\mathrm{pu}_{2}$ could represent $/ \mathrm{p}^{\mathrm{h}} \mathrm{u} /$ and $/ \mathrm{bu} /$, or any more secure evidence for the latter value than a problematic non-Greek word of unknown etymology, the interpretation of $\mathrm{da}-\mathrm{pu}_{2}$-ri-to-jo as /daburint ${ }^{\mathrm{h}}$ oio/ should be rejected, and an explanation for this term sought which is compatible with $p u_{2}$ representing only $/ \mathrm{p}^{\mathrm{h}} \mathrm{u} / .^{79}$

Alternations between voiceless, voiced, and aspirated stops in words of non-Greek origin are in fact relatively common, ${ }^{80}$ so that an alternation between classical $-\beta$ - and Mycenaean $\mathrm{pu}_{2}=/ \mathrm{p}^{\mathrm{h}} \mathrm{u} /$ in a non-Greek loanword is in principle unproblematic; various different non-Greek phonemes could be interpreted in Greek in such a way as to produce these two different spellings, e.g. a voiced bilabial fricative $/ \beta /{ }^{81}$ or a voiced aspirate $/ b^{\mathrm{h}} /{ }^{82}$ Either of these could easily be perceived (at least for orthographic purposes) as closest to $\mathrm{pu}_{2} / \mathrm{p}^{\mathrm{h}} \mathrm{u} /[+$ labial, +aspiration, \pm voicing] in a Mycenaean context (especially

\footnotetext{
${ }^{76}$ Most classical Greek examples of /b/ originate from labiovelars before back vowels (still preserved in Mycenaean) or from epenthesis of */-mrV-/, */-mlV-/ > /-mbrV-/, /-mblV-/. It is unclear whether the latter had already taken place in Mycenaean, since there are no secure examples of terms either with or without this epenthesis. Although it might be expected to have occurred at a similar time to the epenthesis of */-nrV-/ > /-ndrV-/ (which is shown to have already taken place by terms such as $a$-di-ri-ja-pi /andriamp $\mathrm{p}^{\mathrm{h}} \mathrm{i} /<^{*} / \mathrm{anr}-/$ ), it is possible that a transitional [b], even if pronounced, might be less likely to be perceived as fully phonemic than a transitional [d], since only the latter would correspond to a pre-existing phoneme (Thompson 2005:108-9). In any case, there are no secure LB attestations of /b/ (Hajnal 1993:110-2; Thompson 2005:109-11).

${ }^{77}$ E.g. Witczak 1993a:166.

${ }^{78} \mathrm{Cf}$. the lack of LB voiced velar and labiovelar series, and the complete lack of voicing distinction in CS. It is probable that the LA signs which became the LB $d$-series had a different phonetic value, such as a lateral or a dental fricative, which was interpreted as /d/ in Greek: see Lejeune 1958b:327-8; Palaima-Sikkenga 1999:601-2; Davis 2014:204-14; Steele 2014.

${ }^{79} \mathrm{Cf}$. Lejeune 1972b:96; Ruijgh 1967:28, n.30.

${ }^{80}$ Furnée 1972:167-78 gives a list of possible alternations between classical $\pi, \varphi$ and $\beta$ in 'pre-Greek' words.

${ }^{81}$ Yakubovich 2002:109; cf. Davis 2014:214-20.

${ }^{82}$ Thompson 2005; Jiménez Delgado 2008:78.
} 
given the rarity of $/ \mathrm{b} /$ at this period) but to $\beta / \mathrm{b} /[+$ labial, \pm aspiration, +voicing] in a classical Greek context. Alternatively, if da-pu, - ri-to is related to (-)AB51-29-27/(-)DU$\mathrm{PU}_{2}-\mathrm{RE}$ ( $\left.\mathrm{p} .29, \mathrm{n} .72\right), \mathrm{pu}_{2}$ could be explained as a traditional spelling retained from LA, whatever AB29 in fact represented. Whether this word was actually heard and pronounced by Mycenaean Greek speakers as /dap ${ }^{\mathrm{h}}$ urint $^{\mathrm{h}}$ oio/ or as, e.g., /daßurint ${ }^{\mathrm{h}}$ oio/ or /dab ${ }^{\mathrm{h}}$ urint $^{\mathrm{h}}$ oio/ is unreconstructable but, ultimately, not of great importance for the analysis of $\mathrm{pu}_{2}$; any LB sign could in principle stand in for a phonetically similar nonGreek phoneme in loanwords, foreign PNs, etc. What is important for the purposes of this chapter is that not only is it not necessary to assume, based on da-pu $\mathrm{z}_{2}$-ri-to-jo, that $\mathrm{pu}_{2}$ can represent /bu/, it is both more economical and more in accordance with what is known about the LB script to assume that in structural terms (i.e. regardless of what non-Greek phonemes it may sometimes have been used to represent) it stood only for $/ \mathrm{p}^{\mathrm{h}} \mathrm{u} /$.

To answer the question of whether LB is likely to have had other signs for aspirated stops, it is first necessary to consider why $p u_{2}$ itself existed. As the sign was inherited, any explanation of its origins must depend on our view of the sound-values of LA and their relationship to Minoan phonology. The lack of any other distinction of aspirated consonants in LB suggests very strongly that LA did not systematically distinguish the feature of aspiration, whether or not this is to be taken as evidence that aspiration was non-phonemic in the Minoan language (and likewise for voicing), ${ }^{83}$ so that AB29 is unlikely to have represented $/ \mathrm{p}^{\mathrm{h}} \mathrm{u} /$. As in $d a-p u_{2}$-ri-to-jo, it presumably represented a non-Greek phoneme which was reinterpreted as $/ \mathrm{p}^{\mathrm{h}} /$ in the process of adapting LA to LB in order to write Greek. Various forms of secondary articulation, such as palatalisation,

\footnotetext{
${ }^{83}$ The LA ' $d$-series' probably did not represent voiced dentals (p.31, n.78).
} 
glottalisation, or prenasalisation, have been suggested as the Minoan feature underlying LB $p u_{2}$, but none seems especially plausible; ${ }^{84}$ the suggestion of Davis 2014:214-20 that AB29 represents a bilabial fricative $/ \varphi, \beta /$ would fit well with one possible explanation

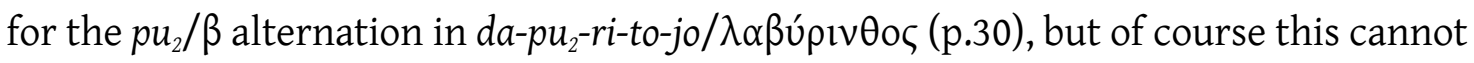
currently be proven any more than any other hypothesis concerning Minoan phonology. Regardless, however, of the actual sound-value of AB29, there seems a reasonably high probability that a series of other similar LA signs existed and that these would have been similarly interpreted in LB, so that LB signs for $/ \mathrm{p}^{\mathrm{h}} \mathrm{a} /, / \mathrm{p}^{\mathrm{h}} \mathrm{e} /, / \mathrm{p}^{\mathrm{h}} \mathrm{i} /$, and/or $/ \mathrm{p}^{\mathrm{h}} \mathrm{o} /$ are fairly strong possibilities. Aspirated dentals, velars, or labiovelars, on the other hand, appear much less likely. If the Minoan feature represented by AB29 was both a recurring one within the Minoan phonological system and systematically represented by LA, and if this was consistently interpreted in Greek as equivalent to aspiration, we would expect a more systematic representation of this feature to be present in LB. Of course, further sporadic instances of the feature expressed by AB29 may have occurred in LA - for instance, if this were a bilabial fricative as suggested above, we might expect to find more fricatives in other articulatory positions - but it is also possible that these might have been differently interpreted in $\mathrm{LB} .^{85}$ On balance, the chances of finding signs for $/ \mathrm{t}^{\mathrm{h}} /, / \mathrm{k}^{\mathrm{h}} /$ or $/ \mathrm{k}^{\mathrm{wh}} /$ seem low; while the demonstration that $p u_{2}$ does not represent $/ \mathrm{bu} /$ also shows that there is no good reason to expect the existence of any further signs specifically marking voicing.

\footnotetext{
${ }^{84}$ L.R. Palmer 1955b:42's suggestion of palatalisation is based on a comparison with $r a_{2}$ and $t a_{2}$, which show a completely different use and distribution in LB (p.48ff), while Stephens-Justeson 1978:281's suggestion of glottalisation is based purely on typological grounds. Melena 1987b's prenasalisation hypothesis is based primarily on his analysis of signs $*_{22}$ and $* 56$, particularly their possible alternations with both $p$ - and $m$ series signs. This will be discussed in more detail in Chapter $2\left({ }^{*} 22:\right.$ p.91; ${ }^{*} 56:$ p.119ff); here it suffices to say that $\mathrm{pu}_{2}$ itself has no such alternations (as Melena 1987b:227 admits).

${ }^{85}$ E.g. Davis 2014:204-14 argues for the $d$-series representing dental fricatives (but cf. Steele 2014).
} 


\subsubsection{Labialised consonants}

These fall into two main groups according to the sign's initial consonant: labialised dentals $\left(d w^{-}, t w^{-}\right)$and nasals $\left(n w^{-}\right)$, each of which will be examined separately.

\subsubsection{Labialised dentals}

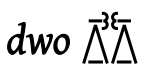

This sign is found 11-12 times at Knossos, Pylos, and Thebes; ${ }^{86}$ its value is shown by alternations with the sequences do-wo and $d u-w o$ (e.g. in the MN wi-dwo-i-jo $\sim$ wi-do-wo-ijo, wi-du-wo-i-jo ${ }^{87}$ and by its appearance alone as the numeral 'two'. ${ }^{88}$ Morphologically $d$ wo consists of two wo signs $\left(\vec{\triangle}^{3}\right)$, with the second reversed. It is usually accepted that this sign was invented based on the sign wo in a Greek-speaking context: 'two wo's' = / duo wo $/=d w o,{ }^{89}$ with the inversion of the second wo presumably intended to avoid confusion with wo-wo (as which this sign was originally read, until Risch 1957 identified it as dwo).

However, Consani 1996 and Davis 2014:195, n.1128 both argue that dwo was inherited from AB118 $\bar{\Delta} \Delta$ (a syllabogram and metrogram), the antecedent of LB L only): L would continue AB118's metrographic use and dwo its syllabographic use. The LB creation would therefore be not $d$ wo but wo, based on the same - but reversed - analysis of the sequence /d(u)wo/ as two wo's, so that a new sign wo was created from half of the

\footnotetext{
${ }^{86}$ I do not regard the possible example on MY Oe 111.2c-a (read dwo-me[ ]jo-i by SCM) as likely; based on autopsy I read ți (cf. TITHEMY).

${ }^{87}$ wi-dwo-i-jo: PY Ep 539.12, Eb 1186.[A]; TH Uq 434.13. wi-du-wo-i-jo: PY Jn 415.3. wi-do-wo-i-jo: PY Ae 344, An 5.2. The Pylian examples probably refer to at least three different people (Nakassis 2013:406).

${ }^{88}$ PY Eb 338.B, Eo 278, Ub 1315.3b; cf. du-wo-u-pi (instr.: PY Eb 149.2, 495.1, Ep 613.1.4, 704.7) and the related MN dwo-jo /Dwoios/ (KN V(3) 492.1, X 8126) du-wo-jo(-jo) (PY An 656.11, Jn 750.12).

${ }^{89}$ Lejeune 1958f:261-2, n.23; cf. Meißner-Steele forthcoming [2017].
} 
sign $d w 0 .{ }^{90}$ This proposal is, however, not supported by the palaeography of the signs in question. $d w o, A B 118$, and $\mathrm{L}$ are structurally similar in having two upright triangular elements, but differ significantly in details (the two triangles are always joined by a horizontal in AB118 and L; L almost always has a central vertical, also seen in some examples of $\mathrm{AB} 118$, but never in $d w o$ ). $\mathrm{L}$ and $d$ wo cannot palaeographically be variants of the same LB sign, nor is there any parallel for a single LA sign diverging to this extent into a separate LB syllabogram and ideogram.

\section{Imageremoved for copyright reasons}

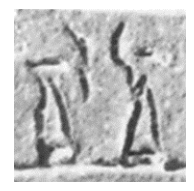

dwo: PY Ep 539.12

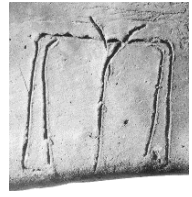

L:

PY Ja 74

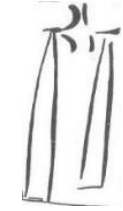

KN Fh $360 . b$

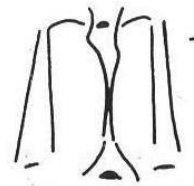

KN Oa 730

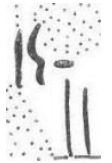

$\mathrm{KN}$ V(3) 492.1

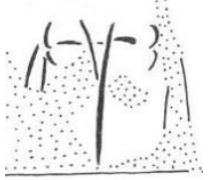

$\mathrm{KN} O g 5515^{91}$

It is both more straightforward and more in accordance with the palaeographic facts to view $d$ wo as an LB creation unrelated to AB118/L. This creation was quite probably motivated at least partly by the ability of such a sign to represent the numeral 'two': $d w o$ 's use in this way is the only LB example of a single sign being used to represent a

\footnotetext{
${ }^{90}$ Cf. Melena 2014b:85, n.112.

${ }^{91}$ Images: AB118: GORILA; PY: MER; KN: COMIK.
} 
word, which the Mycenaean scribes appear generally to have avoided (Melena 2014b:125), suggesting that this was felt to be a particularly special case. The only terms in which $d w o$ is attested apart from $d w o$ and $d w o-j o(p .34, n .88)$ are the obscure MN ma-si-dwo and two terms probably containing the suffix /-wos-/ (> /-woh-/ intervocalically): e-re-dwo-e, probably a perfect participle in /-woh-es/ (m.nom.pl.), ${ }^{92}$ and the MN wi-dwo-i-jo, probably /Widwohios/ <*/Wid-wos-ios/, based on the perfect participle of */wid-/ 'see'. Although the suggestion of Palaima-Sikkenga 1999:605 that $d w o$ was invented in order to represent terms such as these containing this highly productive suffix seems less plausible as a primary motivation than the representation of 'two', the sign's morphological usefulness in this regard may well have been a contributing factor to its creation and/or subsequent use.

\section{$d w e$ 2Ps}

This sign has at least 16 examples at Knossos, Pylos, and Thebes, 13-14 of which are in the term te-mi-dwe (dual te-mi-dwe-te, plural te-mi-dwe-ta) /termid-went-/, 'provided with endings' (probably referring to the attachment of the spokes to the rim); ${ }^{93}$ its value is shown by the alternative spelling te-mi-de-we-te (PY Sa 1266.a)..$^{94}$

$d w e$ has no known LA equivalent, and was most probably created within LB; the sign's structure, with the 'arms' of the usual Knossian form and of the single Theban example

\footnotetext{
${ }^{92}$ Killen-Olivier 1968:123. As this appears in the heading of two personnel tablets, KN As(1) 604.1 and V(3)

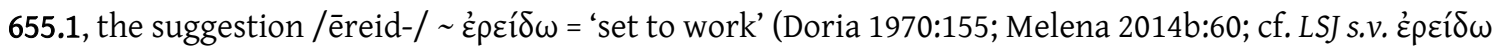
II.2), although tentative, seems contextually plausible.

${ }^{93}$ Crouwel 1981:81-8.

${ }^{94}$ Lejeune 1958a:338-9. Cf. ]-mi-we-te, possibly te-mi-we-te (KN Sg 1811.3); if this is correct, -we- is probably either an error for - $d w e$ - or the result of a different syllabification, /termid|went-/ rather than /termi|dwent-/ (Doria 1962:651; Lejeune 1958f).
} 
consisting of two small mirrored we-shapes (cf. 2 we), suggests that this could have been created by analogy with $d w_{0}$ (the straight strokes at the sides of the Pylos and KN So 894.1.3 forms are probably a simplification of this original form). It is possible that the body of the sign could also have developed from two mirrored we's, with their lower parts simplified to verticals. ${ }^{95}$

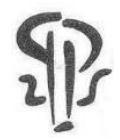

KN So(2) 4433.a

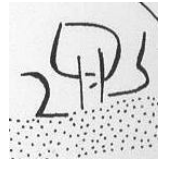

TH Wu 99.及

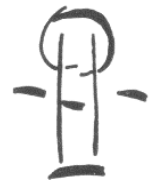

KN So 894.3

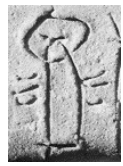

PY Sa $791^{96}$

As $d w e$ is almost exclusively attested in a single term found repeatedly throughout certain tablet series (KN So(1)- and So(2)-series, H130 and H131; KN So-series, unknown hand(s); PY Sa-series, H26), it seems highly probable that the sign could have been invented in order to facilitate the more efficient writing of this repeated term. Note that, as discussed above in the case of $d w o$, /termid-went-/ also contains a heteromorphemic sequence involving a highly productive Mycenaean suffix, in this case /-went-/: its morphological usefulness in this respect may therefore likewise have contributed to its creation and/or continuing use, although it cannot be determined whether either of the other terms in which dwe appears also contained this suffix (]mi-dwe, MN; $a-d w e-e$, obscure).

\footnotetext{
${ }^{95}$ Meißner 2013:10.

${ }^{96}$ Images: Knossos: COMIK; Thebes: TFC 3 ; Pylos: MER.
} 
twe B

There are only 6-7 attestations of this sign, all of them in H130's KN So(1)-series or on other unattributed So tablets, and all certainly or probably in the adjective o-da-twe-ta /odatwenta/ (neuter plural), ${ }^{97}$ "provided with teeth ${ }^{98}$ (the alternative to te-mi-dwe when describing wheels). ${ }^{99}$ Three other spellings of this adjective are also attested o-da-tu-we-ta (So 894, -), o-da-ku-we-ta (So 4435.B, H128?; L 8̣70, H114?), and o-da-ke-we-ta (So(2) 4446.1, H131; Sg 1811.59.6, -) - the last two representing /odakwenta/. ${ }^{100}$ Thus, when $o-d a-{ }^{*} 87-t a$ was identified as the same term, it was debated whether this sign should be transcribed twe or kwe, ${ }^{101}$ twe eventually being agreed upon at the Salamanca Colloquium..$^{102}$

However, since we do not know how H130 would have spelt o-da-twe-ta in full, it is strictly speaking not certain whether this represents /odatwenta/ or /odakwenta/, and the choice of the former rests on the assumption that if a single sign were required to represent /kwe/, this function could be fulfilled by qe $/ \mathrm{k}^{\mathrm{w}} \mathrm{e} /$. Firm evidence for this is, however, lacking: Melena 2014b:40-1 gives several possible examples of - $q$ - representing homomorphemic $/ \mathrm{kw} /{ }^{103}$ but none for heteromorphemic $/ \mathrm{k}-\mathrm{w} /$, which is always spelt

\footnotetext{
${ }^{97}$ ]twe-te (So 8251.a) may be /odatwente/, dual.

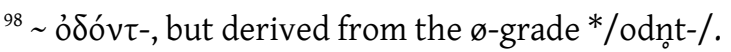

${ }^{99}$ The only possible exception is KN X 7565: ma-twe-to[ is given as a possible (but 'difficult') suggestion by COMIK and $K T^{5}$, but their actual reading ma-ze-to-[ seems far more plausible.

${ }^{100}$ The form in /-k-/ may have arisen through dissimilation from the first dental (Lejeune 1997a:30-1),

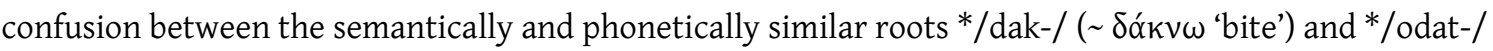
(T. Meißner, pers. comm.; cf. the similar suggestion of CDE regarding the origin of ó $\delta \alpha^{\prime} \xi$ ), or a combination of the two.

${ }^{101}$ E.g. Lejeune 1958c:36.

${ }^{102}$ Ruipérez 1972b:xvi.

${ }^{103} i-q 0$ 'horse' (and derivatives, e.g. i-qi-ja 'chariot'); the root qe-r-<*/gh $w^{h} h_{1} r-/ \sim \theta \eta \dot{p}$ 'wild animal', and the root-qa-<*/kweh ${ }_{2}-/ \sim \pi \dot{\varepsilon} \pi \bar{\alpha} \mu \alpha \mathrm{l}$ 'own'. It is, however, not generally agreed whether the - $q$ - in i-qo represents /-(k)kw-/ (Melena 2014b:40) or a development of this to a labiovelar /-(k)k $\mathrm{k}^{\mathrm{w}}-/$ (DMic). The most

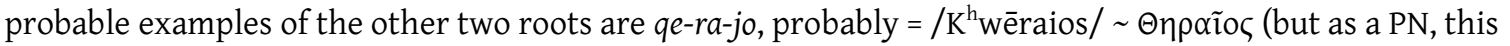


$-k V-w V-.^{104}$ Arguably, $q$ - might still seem an easier choice to represent $/ \mathrm{kw} /$, whether homomorphemic or heteromorphemic, than an entirely separate sign, but in the absence of firm evidence this is no more than an assumption. A further possibility to be considered is whether the process of affrication of $* / \mathrm{tw} /$ (which by the classical period had become /s-/ initially and /-ss-, -tt-/ medially) ${ }^{105}$ could have already begun in Mycenaean, resulting in the creation of a special sign to represent this affricate pronunciation. It seems, however, most likely that this change had not yet begun, ${ }^{106}$ although this cannot be certainly proven; but in any case, although the precise mechanism of the changes affecting */tw/ is not known, the most likely intermediate stage would be */ts $/{ }^{107}$ and would in all probability therefore be represented by LB $z-.^{108}$ The distribution of twe - found in a single scribal hand at what is not likely to be an especially late date compared to the other LB texts ${ }^{109}$ - does not particularly suggest that it marks the result of a sound-change. The value twe, employed purely to represent the sequence /twe/ more efficiently, therefore still seems the most plausible option, if not quite as secure as the values $d w e$ and $d w o$.

is not entirely secure), and mo-ro-qa, probably = /mo(i)ro-kkwās/ 'owner of a plot of land' (but the reconstruction of this root with * $/ \mathrm{kw}-/$ rather than $* / \mathrm{k}^{\mathrm{w}}-$ / is also not entirely secure: $C D E$ s.v. $\left.\pi \varepsilon^{\prime} \pi \bar{\alpha} \mu \alpha \mathrm{l}\right)$. ${ }^{104} \mathrm{As}$ in o-da-ke-we-ta/o-da-ku-we-ta /odak-wenta/; cf. te-tu-ko-wo- $a_{(2)} /$ tetuk ${ }^{\mathrm{h}}$-woha/.

${ }^{105}$ Lejeune 1972f:82-3.

${ }^{106}$ Jiménez Delgado 2004.

${ }^{107}$ Grammont 1946:198.

${ }^{108}$ On the $z$-series, see p.11, n.22.

${ }^{109}$ Regardless of whether the early or late dating is adopted for the majority of Knossos tablets (on which see p.232), they still are significantly earlier than the Pylos tablets and many of the Thebes tablets (p.243). 
Two possible LA antecedents have been suggested for twe. AB87 (GORILA) is attested only on HT 126.b2-3: ${ }^{110}$

Imageremoved for

copyright reasons

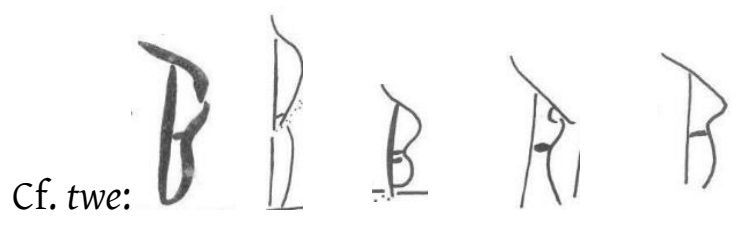

There is no evidence that AB87 was ever used as a syllabogram, since the first example here, at least, is clearly ideographic. Additionally, although twe could, morphologically, be a continuation of $A B 87$, there are potentially significant differences between the form of the first AB87 in particular (with two deep curves arranged near the top) and twe.

A305 is attested as both a syllabogram and an ideogram/abbreviation, and there are some examples which are almost mirror images of twe (Melena 2014b:62, n.73; image: GORILA):

\section{Imageremoved for copyright reasons}

As sign-reversals are not uncommon in LA, it is possible that a form corresponding in orientation to twe existed but is not (yet) attested (indeed it might be tempting to identify AB87 as this mirror-image form of A305, except for the latter's probable

${ }^{110}$ Images: HT 126b: GORILA; twe: COMIK (KN So(1) 4430.b, 4432, 4436.1, 4440.b, 4441). 
attestation on HT 126.1). ${ }^{111}$ It is therefore possible that twe was inherited from one of these LA signs but ultimately retained only by a few scribes at Knossos. However, since neither of the possible LA equivalents is completely convincing and since these all are fairly simple forms, capable of arising independently (cf. the existence of these two fairly similar but apparently unconnected LA signs), it is equally possible that twe was an LB innovation. The sign's distribution also seems to fit better with this explanation: the relative frequency of /odatwenta/, /odakwenta/ on chariot tablets provides a motivation for reducing the number of signs required to write it, especially with $d w e$ (attested five times in the term te-mi-dwe-ta/te in H130) potentially providing a model for the invention of twe. A recent invention by a single scribe would also better explain why none of the other scribes working in the same building and on the same aspect of administration had apparently adopted it (not to mention why it did not come to be widely used in a similar way to $d w e$ ).

\section{two 荨}

This sign occurs only on a single tablet, PY An 261.2-.5 (H43), in the MN o-two-we-o (gen.); cf. H1's o-to-wo-<we->o (Un 616 v.4; An 261 v.7: o-to-wọ[-o); o-tu-wo-we (nom.: Jn 658.7, H21; 725.5, H2) and o-to-wo-we-i (dat.: Vn 851.9, H12). The identity of o-two-we-o and o-to-wo$<w e->0$, on which the identification of two is based, ${ }^{112}$ is guaranteed, since An $261 r$. and $v$. and Un 616 all clearly refer to ke-ro-si-ja headed by the same four individuals (a-pi-jo-to, ta-we-si-jo, a-pi-qo-ta, and o-two-we-o/o-to-wo-<we->o); o-tu-wo-we, at least, is probably the same person (Nakassis 2013:329-30). The name is generally interpreted as /Ort ${ }^{\mathrm{h}} \mathrm{W}$-ōwēs/

\footnotetext{
${ }^{111}$ No CH correspondences are suggested by CHIC:19 or Younger 1996-1997:396. CH 048 if has a broadly similar form, but as this sign's central 'arrow' does not appear in either of the potentially related LA signs or in LB twe, a connection is probably not likely.

${ }^{112}$ Mühlestein 1956:45; Doria 1959. NB that these both assume two and ${ }^{*} 66$ 南 $=t a_{2}$ to be variants of the same sign; the two were distinguished by L.R. Palmer 1961:58 and Lejeune 1971a.
} 
$</$ Wort $^{\mathrm{h}} \mathrm{w}$-ōwēs/ by regressive dissimilation ( ỏ $\rho$ ós, oũc). The fact that this sign is found only on a single tablet on which the same name is repeated four times by one scribe, and that it is spelt differently even by the author of the verso, strongly suggests that this was an idiosyncratic invention by H43, perhaps even specifically on this tablet, in order to simplify the repeated writing of this name. ${ }^{113}$ Very probably the existence of $d w o$ (which is attested in three different hands at Pylos, though not in H43) influenced the creation of this sign.

\subsubsection{Labialised nasals}

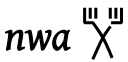

This sign is found 26-27 times at Knossos, Pylos, Mycenae, and Thebes; ${ }^{114}$ the only interpretable term in which it appears is the adjective pe-ru-si-nwa(-o) 'last year's' (/perusinwa/,f.nom.sg. and n.nom.pl.; perhaps also one example of /perusinwai/, f.nom.pl.; /perusinwāhōn/, f.gen.pl.). This is also spelt pe-ru-si-nu-wa, confirming the sign's value as nwa nu-wa; pe-ru-si-nu-wo (n.nom.sg.) is also attested. This term

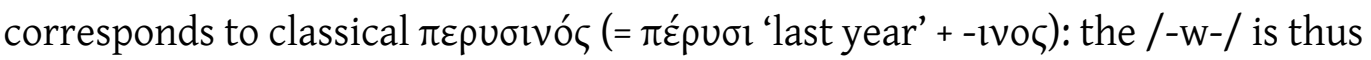
unexpected, but could perhaps have arisen by analogy with the opposing adjective ne-wo /newos/ 'new' (both are found in the KN So-series). ${ }^{115}$

\footnotetext{
${ }^{113}$ Lejeune 1971a:336-7 refers to an observation by E.L. Bennett that on An 261 r.1 -tu-wo- (H1) is over [[-two-]], showing a correction of H43's idiosyncratic spelling. However, the presence of an erasure is mentioned only in PT II, not in PTT or PoN IV, and although possible traces are visible in the MER photograph, they are not legible.

${ }^{114}$ The supposed example from Bernstorf, Germany (Gebhard-Rieder 2002) is excluded from this discussion since the status of this inscription is highly dubious (Harding 2007:52; Hughes-Brock 2011:102-7).

${ }^{115}$ Lejeune 1958c:36-7.
} 
nwa differs significantly from the labialised dentals not just in its initial consonant but also in its origin and use. It has a reasonably well-attested $\mathrm{CH}$ equivalent, $006{ }^{\text {" }} \mathrm{X}^{\mathrm{m}}$ (12

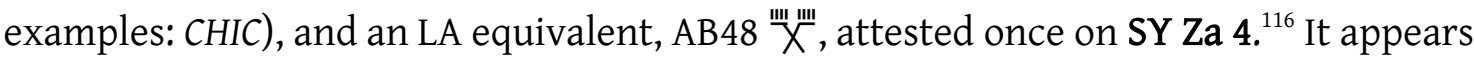
primarily in non-Greek PNs, TNs, a possible theonym (da-nwa) and an ethnic adjective (ti-nwa-si-jo/ja), and the only Greek term in which it is found is one in which the /-w-/ is not etymologically expected and the spelling with nwa has no particular morphological benefit. nwa's use in a Greek context is therefore much less strongly motivated than that of $d w e, d w o$, and twe, especially as /nw-/ was never a permitted onset in Greek, ${ }^{117}$ and it appears that nwa was retained from LA as being useful for more efficiently representing names (probably mainly non-Greek) and occasional vocabulary words containing this sequence; it seems highly unlikely that this sign would have been invented in LB had it not been inherited.

\subsubsection{Summary of labialised signs}

A variety of factors led to the existence of these signs, ranging from retention from LA to invention in LB for the purposes of representing particular terms. In the majority of cases these terms contain important morphological formations (the suffixes /-woh-/ and /-went-/); however, the one-off creation of two for use in a single MN, dwo's additional and perhaps primary function in representing 'two', and the failure of twe to spread as widely as $d w e$ or $d w o$ despite its similar morphological usefulness, all mean that this rather abstract principle of morphological representation (argued by Palaima-Sikkenga

\footnotetext{
${ }^{116}$ Muhly-Olivier 2008. This example is word-medial; $\mathrm{CH} 006$, however, appears relatively frequently in word-initial position (5-6 examples out of the 12 given in $\mathrm{CHIC}$ ).

${ }^{117}$ Meißner 2013:10. The word-initial sequences $n V_{1}-w V_{1}$ - or $n u-w V$ - (in the terms na-wa-to, MN, PY Jn 415.10; ne-we-wi-ja(-o), f.pl. appellative, KN Lc 560.B and PY A-series; nu-wa-(i-)ja, adjective, KN L-series) are therefore probably disyllabic (Lejeune 1958f:259, n.11; Morpurgo Davies 1972:109), though non-Greek words beginning with /nw-/ cannot be ruled out since none of these has a certain Greek interpretation.
} 
1999:605 to be the primary reason for these signs' creation) was probably at most a secondary consideration after the stronger motivating factor of the ability to write frequently-repeated words more efficiently.

Are further labialised signs likely to have existed? It is possible that other signs in $n w-$ could have been inherited from LA alongside nwa; any such signs would, however, probably occur infrequently and (almost) exclusively in non-Greek terms, so that the chances of identifying them seem slight. Given that the vowels of $d w o$ and $d w e$ are particularly well-motivated by Greek morphology, and that twe and two both appear to be one-off creations (which may well have been influenced by the existence of $d w e$ and $d w o$ ) further dentals $d w a, d w i, t w a$, and twi are probably unlikely to have been created within LB. ${ }^{118}$

In principle, it cannot be completely excluded that the LB creations of $d$ we etc. could have been modelled on inherited signs, such as $d w a$ or twa, or that labialised versions of other consonants could have been inherited (any of $k_{-}^{-119} \mathrm{~m}_{-}^{-}, p^{-}, r_{-}^{-}$, or $\mathrm{s}^{-}$; labialised versions of $j^{-}, q^{-}, w^{-}$, or $z^{-120}$ are phonetically implausible). However, the view that more such signs are likely to have existed is based on the assumption that the LB structure of core $C V$ signs plus extra $C w V$ and $C y V$ signs goes back to three LA consonantal series, representing a Minoan phonemic distinction between plain, labialised, and palatalised consonants: thus, $\mathrm{LB} C W V$ signs would originally have represented Minoan $/ \mathrm{C}^{\mathrm{w}}-/$. This

\footnotetext{
${ }^{118}$ If twe was inherited from LA, then further similar signs in tw- could have been inherited as well in a similar way to $n w a$, but would probably not have been widely used given twe's restricted distribution. ${ }^{119}$ If /-kw-/ could not be represented by the $q$-series: see pp.38-9.

${ }^{120}$ Whatever the actual value of the $z$-series (p.11, n.22), it mostly represents developments from palatalised consonants $(* / \mathrm{ky} / \mathrm{etc}$.) and $* / \mathrm{y} /$, so that a sequence $-z w$ - would require two original adjacent semivowels just as -jw-would.
} 
hypothesis, originally suggested by L.R. Palmer $1955 \mathrm{~b}: 38,{ }^{121}$ has recently been reviewed by Davis 2014:193-5 and 236-8. Davis adds a typological argument based on the 'universal' that 'Innovations that produce overrepresentations of highly-marked sounds, while ignoring underrepresentations of less-marked sounds, are almost non-existent in the writing systems of the world'. ${ }^{122}$ Since palatalised and labialised consonants are both more highly marked than either aspirated or voiced stops and (with the exception of the labiovelars) non-phonemic in Mycenaean Greek, this implies that signs representing them could not have been invented in LB without signs for the less marked aspirated and voiced stops first existing.

Employing this typological universal, however, ignores the attested palaeographic facts regarding the labialised signs, ${ }^{123}$ namely that the majority are most likely to have been created in LB; only $n w a$ and the core $q$-series (representing $/ \mathrm{k}^{\mathrm{w}} /$; all of these apart from qo have known LA counterparts) were certainly inherited. This is hardly sufficient grounds to prove the systematic distinction of such a feature at all points of articulation, particularly as labialised velars and nasals are amongst the most typologically common types of labialised consonant phonemes (a survey of UPSID showed that over $50 \%$ of languages with phonemic labialisation had only labialised velars and/or nasals). ${ }^{124}$ It is perverse to employ a (so-called) universal in preference to the attested data. Moreover, this universal is based on phonological grounds, referring to single phonemes such as

\footnotetext{
${ }^{121}$ See also L.R. Palmer 1963b:38-40, and the similar phonemic reconstruction of 'Pre-Greek' in EDG:xvi-xvii and Beekes 2014:4.

${ }^{122}$ Cf. Stephens-Justeson 1978:279.

${ }^{123}$ On palatalisation, see pp.59-60.

${ }^{124}$ Cf Meißner 2013:9-10. As nwa also has a CH correspondence, this sign presumably originated in the hypothetical ancestor of LA and $\mathrm{CH}$, in which case it cannot necessarily provide information about the language behind LA (see p.9, n.14). The same may apply at least to qe, the only member of the $q$-series with a possible $\mathrm{CH}$ antecedent (074/075 $\odot \odot:$ CHIC:19).
} 
$/ \mathrm{k}^{\mathrm{w}} /, / \mathrm{n}^{\mathrm{w}} /, / \mathrm{d}^{\mathrm{w}} /, / \mathrm{t}^{\mathrm{w}} /:$ but, again with the exception of the labiovelars, this is not what the LB labialised signs represent, since they stand for clusters of two phonemes (/nw-/, $/ \mathrm{dw}-/, / \mathrm{tw}-/)$. The overriding principle behind their creation and use in LB is not phonemic representation, but efficiency in writing combinations of phonemes, and so a universal based on phonology simply does not apply. There is, therefore, very little evidence for the existence of complete series of labialised consonants in LA, and consequently for systematic Minoan labialisation. Without such evidence, there are no particularly good grounds to suppose that further signs of this type were inherited by LB, although further one-off signs, similar to $n w a$, cannot be entirely excluded.

Could further $\mathrm{CwV}$ signs have been created in LB? The question is whether such sequences are likely to have been sufficiently common in Mycenaean Greek, particularly in the context of frequently-repeated terms and/or those containing important morphological elements, to motivate the creation of new signs. In order to address this question, a survey of all possible instances of $/ \mathrm{Cw} /$ clusters with $k-, m_{-}, p-, r-$, and $s^{-}$was conducted; ${ }^{125}$ although its results cannot be regarded as definitive - further examples of these clusters could exist in obscure terms, which are excluded here, while if any of the undeciphered signs do stand for clusters of this sort this might skew the results - they may at least provide an indication of the relative probability of different $/ \mathrm{Cw} /$ clusters occurring.

Under the above criteria, / kw/ and /rw/ display the best potential motivation for the creation of new signs. The former is attested in one adjective (o-da-ke-we-ta/o-da-ku-we-ta

\footnotetext{
${ }^{125}$ Via a DAMMOS search for sequences of the type $C V_{1}-w V_{1}$ and $C u-w V$, followed by comparison with DMic, $T F C^{1}$ 's Glossary, and/or individual tablets' publications.
} 
/odak-wenta/: KN), 1-2 perfect participles (te-tu-ko-wo- $a_{(2)} /$ tetuk $\mathrm{k}^{\mathrm{h}}$-woha/: KN, PY; ]ko-woa /-k-woha/: IK), and one compound (a-pu-ko-wo-ko /ampuk-worgos/: PY); see pp.38-9, however, on the probability that $q$-series signs would be used for $k w V$ sequences rather than that new signs would be invented for them. /rw/ appears in one perfect participle

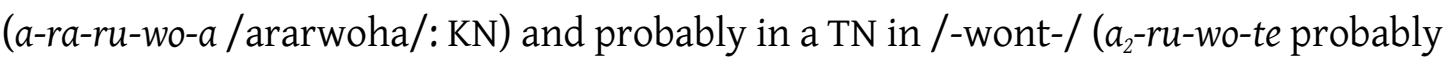
/Hal-wontei/: PY); note in particular the repetition of a-ra-ru-wo throughout the KN Raseries. Of the four possible instances of /sw/, all are probably secondary, since */sw/ > /(h)w-/ initially, /-uw-/ medially in Greek (cf. we-pe-za */(h)wek-pedza/ > /(h)we(p)pedza/ 'six-footed' < */sweks-/), ${ }^{126}$ and none are of particular morphological importance; ${ }^{127}$ there are no known examples of /mw/ or /pw/ at all.

It seems, therefore, that of the possible further labialised values, rwo might have the strongest chance of being invented within LB; kwe and/or kwo are less likely (since it may have been possible to represent these sequences with $q$-series signs) but cannot be entirely excluded, and the same goes for signs in sw-. In all of these cases, the existence of complete series seems highly unlikely, nor would it even be necessary for both $e^{-}$and o-signs to have been created. Even in the relatively strongly-motivated labialised

\footnotetext{
${ }^{126} \mathrm{On}$ */sw-/, see Lejeune 1972f:133-6. DMic interprets this as /hwes-pedza/ (based on e.g. Lejeune 1958d:165) but (as pointed out to me by T. Meißner) this is not a plausible outcome of */sweks-/; the spelling we-pe-za is unproblematic if the */-k-/ has, as seems perfectly plausible, assimilated to the following /-p-/ (cf. other similar examples of assimilation to a following labial, as also discussed by Lejeune, ibid.: e.g. a-di-ri-ja-pi /andriamphi/ <*/andriant-p ${ }^{\mathrm{h}} \mathrm{i} /$ ).

${ }^{127}$ 1) $a$-si-wi-jo/-ja /Aswios, -a/ (KN, PY, MY): ethnic adjective 'Ă $\sigma i ́ \alpha$. Alternations in the length of the first vowel (cf. Hom. 'Āoí

2) wi-so-wo-pa-na /Wiswo-/ (PY): adjective, first element $\sim$ l̆ooc. The exact derivation of this form is debated but it is generally agreed that an intermediate form */wiswos/ is the origin of the classical form (CDE s.v.

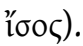

3) pi-sa-wa-ta /Piswātās/ (PY): MN supposedly derived from TN */Piswā/ / /Pīsāal. However, as the reconstruction */Piswā/is almost entirely based on this form, this is rather circular (Chadwick 1968a:64).

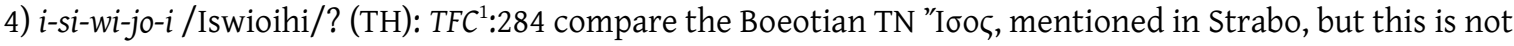
certain.
} 
dentals, the two tw- signs are independent inventions with highly restricted distributions, and the same could well be true of any further labialised signs which might exist.

\subsubsection{Palatalised consonants}

This group includes the palatalised liquids $r a_{2}$ and $\mathrm{ro}_{2}$ and the palatalised dental $t a_{2}$; the sign pte is also generally thought to have originated as a palatalised sign *pye, although I will show that there is reason to doubt this.

\subsubsection{Palatalised liquids}

$r a_{2} \pi$ = /rya, lya/ > /rra, lla/; $\mathrm{ro}_{2} \Phi=/ \mathrm{ryo}, \mathrm{lyo} />/ \mathrm{rro}, \mathrm{llo} /$

These two signs will be discussed together as they largely face the same issues of interpretation.

$\mathrm{ra}_{2}$ has at least 63 attestations at Knossos, Pylos, Thebes, and Mycenae, and $\mathrm{ro}_{2}$ at least 47 attestations at Knossos, Pylos, and Thebes; ${ }^{128}$ both are attested word-medially and finally but not initially. ${ }^{129} \mathrm{ra}_{2}$ has a clear LA antecedent, AB76 \2 SS, and a probable CH correspondence, 069 (CHIC:19); ${ }^{130}$ there is no known LA antecedent to $\mathrm{ro}_{2}$, so although

\footnotetext{
${ }^{128}$ The only example of $r_{2}$ previously identified at Mycenae (e-rọ- $-m e-n a$, Ge 603.2: MT III) is now, much more probably, read e-ne-me-na (SCM; TITHEMY), and is therefore excluded.

${ }^{129}$ The only possible instance of initial $r a_{2}\left(r a_{2}[\right.$, PY An 614.1) is extremely doubtful.

${ }^{130}$ Unlike $\mathrm{ra}_{2}$, both $\mathrm{AB} 76$ and $\mathrm{CH} 069$ are attested word-initially, though AB76 is rare in this position (one example, on ZA 10b.5, out of 44-8 listed in GORILA); 069 also has only one initial attestation (\#041.a) but this is out of just four listed in CHIC.
} 
it cannot be ruled out that chances of attestation may be responsible for this, it is generally regarded as an LB invention. ${ }^{131}$

$r a_{2}$ 's value as /rya, lya/ is shown by several alternations with ri-ja, mainly in agent nouns in */-tria/ describing groups of women in the PY A-series, e.g. me-re-ti-ra $a_{2}(-o) \sim$ me-re-ti-ri-ja */meletriai/ 'flour-grinders'. ${ }^{132} \mathrm{ro}_{2}$ is shown to be /ryo, lyo/ by the alternation of $p o-p u-r o_{2} \sim p o-p u-r e-j o /-j a * / p o r p h$ hr(e)in io-/ 'purple'; ${ }^{133}$ note the resyllabification of /-riV-/ > /-ryV-/ in both cases (Lejeune 1997b:211; cf. p.53 on $\mathrm{ta}_{2}$ ).

There is also, however, one instance of each of these signs alternating with a core $r$ series sign. The MNs ta-ra $a_{2}$-to and ta-ra-to certainly refer to the same individual at Pylos, as both are te-o-jo do-e-ro holding an o-na-to of GRA T 1 from $a_{3}$-ti-jo-qo, ${ }^{134}$ while ku-pa-ro ${ }_{2}$ and ku-pa-ro, found on tablets recording aromatic substances, are clearly both 'cyperus'. ${ }^{135} \mathrm{ra}_{2}$ also occurs in $a-k e-r a_{2}$-te (PY Vn 493.1), generally accepted to be the

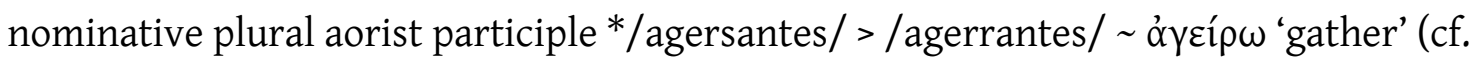

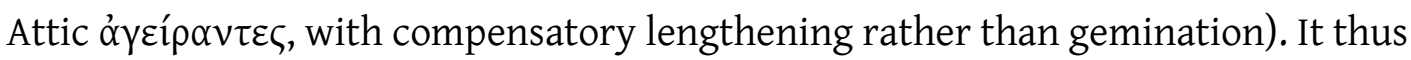

\footnotetext{
${ }^{131}$ Younger 2003:310 suggests a connection between $\mathrm{ro}_{2}$ and $\mathrm{CH} 040$; however, the resemblance between these signs is at best slight, and the more usual comparison of 040 to AB86/*86 is much more plausible (see pp.172-3).

${ }^{132}$ Witczak 1994's interpretation of this sign as $r i_{2}$ assumes the existence of a non-specific doublet and has no supporting evidence.

${ }^{133}$ po-pu-ro $\mathrm{r}_{2}$ dual in /-ō/, KN L 758.a; po-pu-re-ja = n.pl., L 747 (both followed by TELA+PU). po-pu-re-jọ [ (m.nom.pl.?) appears on X 976, plausibly also a textile tablet. The alternation /-io-/ /-eyo-/ is paralleled in other adjectives, e.g. wi-ri-ne-jo wi-ri-ni-jo; these may be two different suffixes or phonetic variants of a single suffix /-(e)io-/ (Killen 1983a:88-9).

${ }^{134}$ ta-ra 2 -to: En- and Eo-series, passim, especially En 74.15; ta-ra-to: Eo 247.6. See Nakassis 2013:380.

${ }^{135} \mathrm{ku}$-pa-ro: : PY Un-series, followed by AROM. ku-pa-ro: KN Ga-series, followed by AROM+PYC, *171, or dry measure symbols; cf. ku-pa-ro-we, '(oil) treated with cyperus' (PY Fr 1203). Probably */kuparyos/ vel sim., with different treatments of a non-Greek vowel and simplification processes producing the various

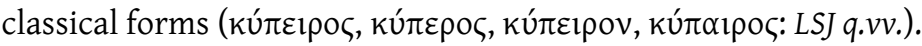


appears that */ry, ly/ > /rr, ll/ in Mycenaean, and that $r a_{2}$ and $\mathrm{ro}_{2}$ therefore came to stand for /rra, lla/ and /rro, llo/ regardless of the sequence's historical origin. ${ }^{136}$ The alternations with $r a$ and ro as well as ri-ja and ri-jo are thus easily explained - since LB does not standardly distinguish geminate consonants, the former are the normal spellings of /rra, lla/ and /rro, llo/ (so that there is no basis for the argument that $\mathrm{ra}_{2}$ and $\mathrm{ro}_{2}$ can stand specifically for $/ \mathrm{la} /$ and $\left./ \mathrm{lo} /\right),{ }^{137}$ while the latter represent conservative spellings and/or pronunciations of $* / \mathrm{ry}, \mathrm{ly} / ;^{138}$ this also fits with the lack of word-initial examples of either sign, since neither*/Ry, Rs/ clusters nor geminates occurred wordinitially in Greek (Lejeune 1972f:124-7, 171). Hence at the time of the LB tablets the terms in $\mathrm{ra}_{2}$ and $\mathrm{ro}_{2}$ listed above had the forms /melet(i)rrai/, ${ }^{139}$ /porph urrō/, and /kuparros/ ( $t a-r a_{(2)}$-to remains obscure; the interpretation /Straton/ should be abandoned).

\footnotetext{
${ }^{136}$ Melena 2014b:66 suggests that the lack of $\mathrm{ro}_{2} \sim$ ri-jo alternations shows that $\mathrm{ro}_{2}$ was created subsequent to this sound change. However, ri-jo is found in similar contexts to $\mathrm{ro}_{2}$ (e.g. word-finally in MNs in /-ios/ or /-iōn/), so that its situation seems similar to that of $r a_{2}$ and $r i-j a$. Since LB did not regularly distinguish geminate consonants, it does not seem likely that a new sign would be created specifically to represent them; the lack of direct $\mathrm{ro}_{2} \sim$ ri-jo alternations is therefore probably due to chance.

${ }^{137}$ Heubeck 1979; Leukart 1992:400-1. The main evidence for this comes from two terms: 1) the Pylian MN $e-k e-r a_{2}-w_{0}$ (nom.: gen. in -no, dat. in -ne), also spelt e-ke-ra-<wo->ne, ]e-ke-ri-ja-wo, and e]-ke-ra $a_{2}-u-n a$ (Nakassis 2013:243-4); this is better assumed to come from a form with original */rya/ or */lya/ (García Ramón 2014) rather than the orthographically difficult etymologies /(h)Ek e(l)lāwōn/ ( Éx $\omega, \lambda \bar{\alpha}$ fóc) or

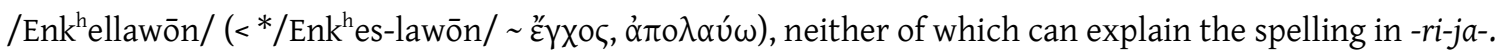

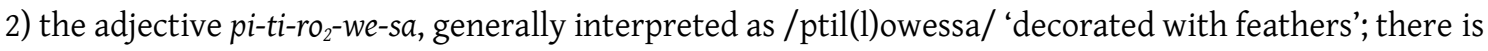

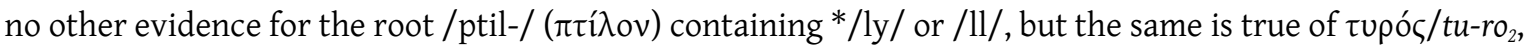
universally accepted to be */turyos/ > /turros/ (this point was made to me by 0 . Simkin). Additionally, since there is no contextual information to demonstrate the precise meaning of this adjective, it is not completely certainly related to $\pi \tau$ í $\lambda o v$. There is thus no good evidence that $r a_{2}$ and $r \mathrm{O}_{2}$ were used for nongeminate liquids, nor indeed any plausible orthographic reason why they should have been. ${ }^{138}$ The precise relationship between the spellings -ti-ri-ja and -ti-ra ${ }_{2}$ for */-tria / has been much debated, with suggestions including variation in pronunciation due to fast/slow speech (Lejeune 1972b:100-1); variant graphic representations of a palatalised geminate /ŕŕa/ (Ruipérez 1972a:161); synchronic dialectal variation (Chadwick 1983:83-6); and different outcomes of an original ablauting paradigm (Heubeck 1979:253; Leukart 1992:401-3). The most plausible explanation, however, is that these simply originated as orthographic variants and that -ti-ri-ja continued in use as a conservative spelling (Gallavotti 1958a; Lejeune 1997b:211-12; Jiménez Delgado 2011).

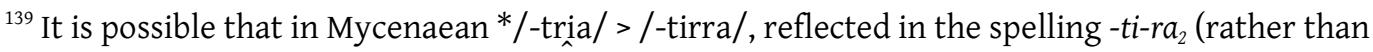

*-ta-ra $a_{2}$ for */-trra/): cf. the operation of similar processes of reanalysis, gemination, and epenthesis in

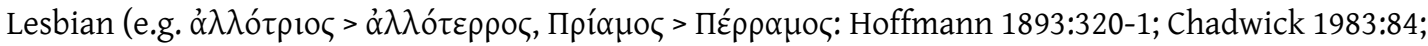


Note the morphological usefulness of both of these signs in representing $a$ - and o-stem nominal/adjectival endings: c. $25 \%$ of $r a_{2}$ 's attestations are in $a$-stem formations (mainly the agent nouns discussed above; also the noun $\left.m i-r_{2}\right)^{140}$ and at least $30 \%$ of $\mathrm{ro}_{2}$ 's attestations in corresponding o-stem formations (in addition to $\mathrm{ku}-\mathrm{pa}-\mathrm{ro}_{2}$ and $\mathrm{po}-\mathrm{pu}-\mathrm{ro}_{2}$, tu-ro ${ }_{2}$ /turro-/ 'cheese', qe-ro ${ }_{2} / \mathrm{sk}^{\mathrm{w}}$ ellō/ 'armbands' (dual)?, and ka-tu-ro $/ \mathrm{k}_{2}$ kant ${ }^{\mathrm{h}}$ ullos/

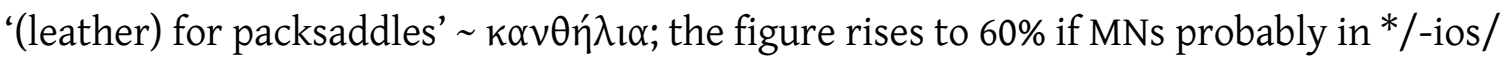
and */-iōn/ are included). This may well have contributed to the retention and relatively widespread use of $\mathrm{ra}_{2}$, and to the (probable) LB creation of $\mathrm{ro}_{2}$.

\subsubsection{Palatalised stops}

\section{$t a_{2}$ 圆 $=/ \mathrm{tya} /($ or $/ \mathrm{ta} /$, or $/ \mathrm{sta} /$ ?)}

This sign is found up to 25 times at Knossos, Pylos, and possibly Mycenae; ${ }^{141}$ it appears word-medially or finally but not initially. $\operatorname{ta}_{2}$ has an LA antecedent, AB66 $\oplus^{142}$ but (unlike $\mathrm{ra}_{2}$ ) no $\mathrm{CH}$ correspondence; however, this may be due to chances of attestation. Although $t a_{2}$ is generally regarded as /tya/ (e.g. Chadwick 1956:238; Docs ${ }^{2}: 391$ ), it has also been argued to be a simple doublet of $t a$ (Heubeck 1979:254-7) or to represent /sta/ (Melena 2014b:78-82).

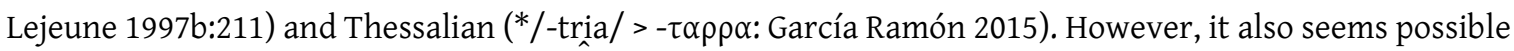
that both -ti-ri-ja and -ti-ra $a_{2}$ are conservative spellings and a pronunciation /-trra/ is therefore simply not reflected in the orthography.

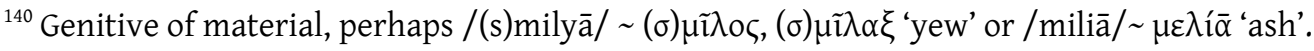

${ }^{141}$ MY Oe 111.4 is read ]-rị-ta $-n i-j a ̣$ by SCM and ]-ki-ni-*5̣ by TITHEMY; from autopsy I read ]-ki-ni-j̣a but ]-tá $a_{2}$-ni-jạ cannot be excluded.

${ }^{142}$ AB66 is primarily attested word-finally (8-9 examples in GORILA) but unlike $t a_{2}$ also has three initial examples (plus an isolated example classed by GORILA as a monosyllabic word, and one uncertain medial example). 
The only relatively secure alternation involving this sign is in a TN at Pylos, variously

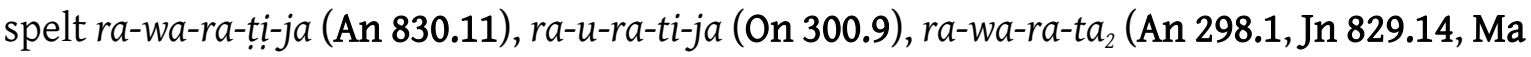
216.1), and ra-wa-ra-ta (An 723.3); cf. pu-ro ra-u-ra-ti-jo (Ad 664) or pu-ro ra-wa-ra-ti-jo (Cn 45.1.2.3b.8b.9.12). ${ }^{143}$ The region in question could evidently be referred to by either a TN (/Laurant ${ }^{\mathrm{h}}$ a?/) or the related adjective (/Laurant ${ }^{\mathrm{h}} \mathrm{ia}$ ?/), ${ }^{144}$ while the main settlement in the region is 'Laurant ${ }^{\text {ian Pylos'. }}{ }^{145}$ Although the identification of $r a-w a-r a-t a_{2}$ with these other forms is secure insofar as the same place is clearly being referred to in all cases, ${ }^{146}$ in principle this could represent an alternative spelling of either ra-wa-ra-ti-ja or ra-wa-ra-ta. The distribution by scribal hand (H1 and a Cii hand write -ti-ja, and H1 also writes -ta; $\mathrm{H} 2$ and $\mathrm{H} 3$ write $-\mathrm{ta}_{2}$, while $\mathrm{H} 21$ and $\mathrm{H} 23$ refer to pu-ro ra-wa-ra-ti-jo) does not show whether the use of $-t_{2}$ reflects a preference for a particular orthography or for the TN vs. the adjective. ${ }^{147}$

The only vocabulary word in which this sign appears, $k_{0}-r o-t a_{2}$, is clearly neuter plural (referring to TELA in the $\mathrm{KN} \mathrm{Ld(1)-series)} \mathrm{and} \mathrm{related} \mathrm{to} \mathrm{ko-ro-to} \mathrm{(an} \mathrm{adjective} \mathrm{describing}$ LANA on MY Oe 106.1 and probably also in the KN Od-series), ${ }^{148}$ but could theoretically be the neuter plural either of ko-ro-to or of a related adjective in /-ios/. The fact that ko-ro-to refers to wool and ko-ro-ta ${ }_{2}$ to cloth might encourage the latter interpretation $\left(k o-r o-t a_{2}=\right.$

\footnotetext{
${ }^{143} \mathrm{NB}$ that an alternation of $-t a_{2}$ and -ti-jo here is excluded, both because $r a-w a-r a-t i-j o / r a-u-r a-t i-j o$ is only found following pu-ro and because the former's appearance in the clearly neuter plural ko-ro-ta ${ }_{2}$ guarantees its $a$-vocalism (Lejeune 1971a:333).

${ }^{144} \mathrm{Cf}$. other similar examples at Pylos, e.g. TN pa-ki-ja-ne, pa-ki-ja-pi, pa-ki-ja-si vs. adjective pa-ki-ja-ni-ja (Heubeck 1979:257).

${ }^{145}$ See Bennet 1998:124-5.

${ }^{146}$ These forms all appear in similar lists of TNs (Lejeune 1972a:44).

${ }^{147}$ A second alternation often identified in Pylian TNs is $a$-[.]-t $a_{2}$ (Ma 397.1) a-si-ja-ti-ja (e.g. Lejeune 1972a:44-6). However, this depends on interpreting the unidentifiable sign in $a$-[.]- $t a_{2}$ as an otherwise unknown sign sya (Lejeune 1972b:91); the condition of the tablet makes even identifying this as a new sign, let alone assigning it a value, highly insecure (cf. p.1, n.4).

${ }^{148}$ Od 485.a ko-ro[, 486.a ]ro-to, 487.a ]to.
} 
'cloth of ko-ro-to type/made of ko-ro-to wool'), ${ }^{149}$ and the best interpretation of both terms seems to be that of Lejeune 1972a:48, that ko-ro-to = / $\mathrm{k}^{\mathrm{h}}$ rōstos $/ \sim \chi \rho \omega \dot{v} v \bar{v} \mu \mathrm{r} / \chi \rho \omega \dot{\iota} \zeta \omega$ 'dyed' and ko-ro-ta $a_{2}=/ \mathrm{k}^{\mathrm{h}}$ rōstia/, '(cloth) for dyeing' (for an adjective in /-(e)ios/ denoting obligation, cf. qe-te-o/jo = / $\mathrm{k}^{\mathrm{w}}$ eitehos, - eyos ( 'to pay'); however, this cannot be regarded as certain. ${ }^{150}$ As the majority of the other terms in which the sign is found are all PNs, they are of little help in settling this question (e.g. MNs in - $t a_{2}$ could be in /-tās/ or /-tiās/, and WNs in /-ta/ or /-tia/). ${ }^{151}$

In the absence of completely secure evidence from $t a_{2}$ 's attestations, the identification of $t a_{2}$ as a doublet of $t a$ relies on the argument that the value /tya/ could not in fact exist, since */ty/ had undergone both waves of palatalisation (e.g. to-so /to(s)sos/ < */totyos/; -we-sa /we(s)sa/ <*/wntt-ya/: Lejeune 1972f:108) and there is no evidence for Mycenaean syncope of */CiyV/ > /CyV/ (Heubeck 1979:254). ${ }^{152}$ None of the proposed interpretations of terms in $t a_{2}$, however, assumes an origin in either of these processes; rather, they are interpreted as terms in */-tia/ (e.g. feminine/neuter plural adjectival endings: written -ti-ja with a non-phonemic glide) which could easily undergo a resyllabification process to /-tya/ (cf. the development of */-tria/ to /-trya/ [p.49]; since the palatalisations of

\footnotetext{
${ }^{149}$ Killen 1979:162-3.

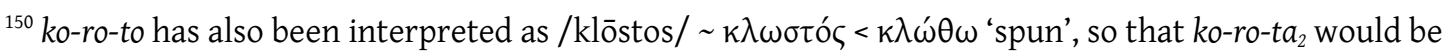
/klōst(i)a/, but 'spun/made from spun wool' seems a redundant description for cloth, while the interpretation / $\mathrm{k}^{\mathrm{h}} \mathrm{rōsta} /$ 'dyed' seems rather imprecise given ko-ro-ta ${ }_{2}$ 's appearance alongside various terms for coloured or decorated cloth (Killen 1979:157-63). On the same basis, though, it is not clear why cloth 'for dyeing' should be delivered and stored alongside finished cloth.

${ }^{151}$ Lejeune 1971a:333-4.

${ }^{152}$ Melena 2014b:79 adds the argument that the lack of word-initial examples of $t a_{2}$ is problematic, since 'the initial position... is a suitable place for palatalised plosives': this assumes incorrectly that such palatalisation would be a secondary demarcative phenomenon. In fact, $t a_{2}$ 's lack of initial examples is perfectly in keeping with the distribution of $r a_{2}$ and $r o_{2}(p p .48,50)$, and $* / t^{(h)} i V-/$ is a very uncommon word-initial sequence in Greek (as Melena 2014b:79, n.103 admits; LSJ lists only a few glosses from

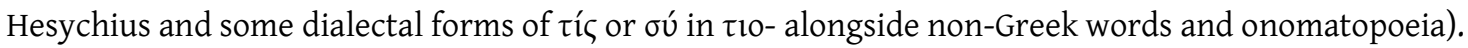


both */ty/ and */t-y/ were complete, these would not then undergo this process). There is thus no linguistic problem with the value $/$ tya/, ${ }^{153}$ nor any good reason based on the structure of the script to assume $t a_{2}$ to be a simple doublet of $t a(p .6) .{ }^{154}$

Despite this, Melena 2014b:78-80 similarly regards /tya/ as problematic and therefore suggests the value /sta/: both proposed interpretations of ko-ro-to/ko-ro-ta ${ }_{2}$ contain /-st-/, and Melena, arguing that ra-wa-ra-ti-ja et al. must end in /-stia(-)/ in order to explain the lack of assibilation, interprets $r a-w a-r a-t a / r a-w a-r a-t a_{2}$ as /Laurāstai/. However, ethnics in -ti-jo/-ti-ja regularly fail to assimilate in Mycenaean even without an inhibiting /-s-/ (e.g. $\left.a_{3}-k u-p i-t i-j o / A i g u p t i o s /\right)$, presumably being protected by analogy with the relevant TNs. ${ }^{155}$ It is also questionable how likely it would be for a complex sign to exist for a cluster which is not even fully represented according to normal LB spelling rules. There appear to be no examples of /-st-/ spelt as $-s V_{1}-t V_{1}{ }^{156}$ if its representation was considered important enough for at least six scribes at two (or three) different sites to use $t a_{2}$, we might expect there to be instances of the cluster being spelt in full. Moreover, we already have evidence for the existence in LA of one sign which (whatever its precise LA value) in LB originally represented /Cya/, namely $r a_{2}$; assuming the existence of a further similar sign is therefore much less difficult than that of a sign for /sta/.

\footnotetext{
${ }^{153}$ The explanation suggested by Chadwick 1983:82, that */ty/ could have been preserved for longer in a sub-standard dialect, raises the question of why such a sub-standard value would have a special LB sign. ${ }^{154}$ The theoretically possible value /tai/ is excluded, since ko-ro- $\mathrm{ta}_{2}$ is neuter plural and some of the PNs ending in $-\mathrm{ta}_{2}$ are nominative (Melena 2014b:80, n.107), while / $\mathrm{t}^{\mathrm{h}} \mathrm{a} /$ has been shown to be unlikely (p.33). 155 Thompson 2008:755.

${ }^{156}$ There are only two generally accepted examples of plene spelling of an /-sC-/ cluster (where $C$ is a

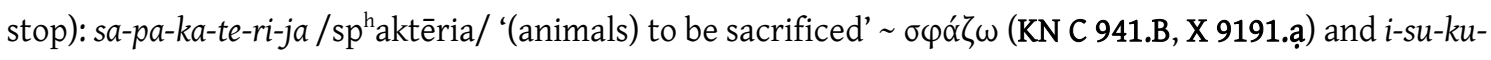
wo-do-to /Isk huwodotos/ (MN) ioxús (KN Fh 348.1).
} 
On balance, then, despite the lack of completely conclusive evidence for the value of $t a_{2}$, /tya/ appears the most plausible value, both on linguistic grounds and considering the structure of the script. In a similar way to $\mathrm{ra}_{2}$, the sign's usefulness in representing caseendings of adjectives and PNs (and, theoretically, nouns) in /-ia/ (or /-iās/) may have contributed to its retention from LA and continued use in LB.

pte

This sign is found 58-63 times at Knossos, Pylos, and Tiryns; it has no known LA or CH correspondences, ${ }^{157}$ and was therefore probably an LB invention (although, as in all such cases, an absence from LA and/or $\mathrm{CH}$ due to chances of attestation cannot be ruled out). Its value is shown by the alternations pte-re-wa pe-te-re-wa (/ptelewās/, '(made) of elm', describing wheels in the KN Se- and So-series) and perhaps tu-ru-pte-ri-ja tu-ru-pe-te[-ri-ja (/struptērias/, 'alum'; tu-ru-pe-te[ on KN X 986 is without context but this restoration seems probable).

pte is unique as the only known LB sign with a $C_{1} C_{2} V$ structure (where $C_{2}$ is a stop rather than a semi-vowel). For this reason, it is generally explained (following Lejeune 1997b:204-5) as originally having the value /pye/ and subsequently becoming /pte/ through the regular Greek sound change */p ${ }^{(\mathrm{h})} \mathrm{y} />/ \mathrm{pt} / ;^{158}$ this must have taken place

\footnotetext{
${ }^{157}$ Two $\mathrm{CH}$ signs, 034 ss $\boldsymbol{s s}$ and $036 \mathrm{M} \boldsymbol{\Lambda}$, bear a slight resemblance to pte, but neither is close enough to make this identification particularly plausible.

${ }^{158}$ I am aware of two previous arguments to the contrary. Ruijgh 1967:53, n.35 suggests that pte noted a

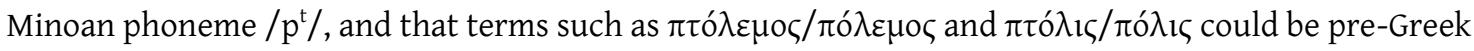
borrowings preserving traces of this phoneme; however, not only is pte apparently absent from LA and infrequent in words of possible Minoan origin (p.58), but both $\pi(\tau)$ ó $\lambda \varepsilon \mu \circ \zeta$ and $\pi(\tau)$ ó $\iota_{\imath \varsigma}$ have probable IE etymologies (CDE s.v. $\pi \tau-)$. Neumann 1996:96-8 argues that pte is an acrophonic derivation from $\pi \tau \varepsilon \dot{\varepsilon} p v \xi$ 'bird' or $\pi \tau \varepsilon \rho o$ $v$ 'wing', with the form of the sign representing a bird's raised wings; this seems neither an especially plausible explanation for the shape of pte nor a secure basis on which to posit its original value.
} 
considerably earlier than the date of the LB tablets, since pte is consistently used to represent /pte/ or $/ \mathrm{p}^{\mathrm{h}} \mathrm{t}^{\mathrm{h}} /$ in roots which do not go back to */py/ (in fact, none of its attestations go back to this sequence). ${ }^{159}$ As Lejeune 1997b:205 remarks, however, 'il faut bien voir que le postulat initial (homogénéité des signes CCV) est arbitraire; nous ignorons tout des circonstances et motivations diverse qui ont présidé à l'institution des «complexes»'. Assuming this sign to have been an LB invention, the question to be asked is therefore what the purpose of a sign *pye would have been.

Palatalised labials could in principle be of use in similar circumstances to the other palatalised signs: *pya and *pyo, if they existed, could have represented the endings of nouns and adjectives in /-ia/ and /-ios/ with stems ending in labials, but this is obviously not the case for *pye. CDE lists no Greek roots in $\pi \tau \varepsilon^{-}<* /$ pye-/, and the most common morphological formation in which this sequence would occur is presumably the second and third persons of verbs with labial stems followed by the suffix */-ye, -yo/

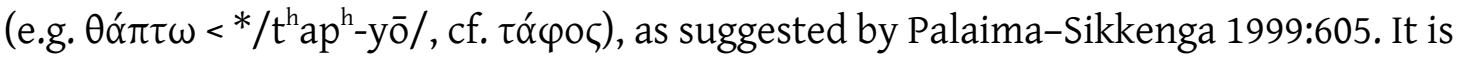
therefore not surprising that we have no examples of */pye/ in Mycenaean, ${ }^{160}$ given the rarity of verbs in LB; equally, this means that we might not expect the creation of a sign to represent a morphological combination found most commonly in verbs. ${ }^{161}$ PalaimaSikkenga 1999:605 argue that the creators of LB would have 'take[n] into account key features of their normal living language, such as the verbal system', and therefore verbs in */-ye, -yo/could have influenced the creation of palatalised signs, regardless of their

\footnotetext{
${ }^{159}$ Lejeune 1972f:79 and 1997b:205.

${ }^{160}$ Secondary /pye/ is probably also not attested: there are no examples of the sequence *pe-je and only two of pi-je. These are in the MN a-pi-je-ta, perhaps /Amphietās/, and pi-je-ra $a_{2} / p^{h}$ ielai/ = pi- $a_{2}-r a / p^{h}$ ihalai/ (both nom.pl., a type of vase); in both cases pi-je probably represents /pi ${ }^{\mathrm{y}} \mathrm{e} / \mathrm{rather}$ than /pye/. ${ }^{161} \mathrm{~F}$. Aurora has pointed out to me that if LB was also used to write longer texts on perishable materials, these could have included a wider range of linguistic categories than the tablets; however, as discussed above (p.22, n.50), it does not seem probable that LB was used in this way.
} 
infrequency in the tablets; but as already argued throughout this chapter, the creators and users of LB were concerned not with a linguistically optimised system for representing Mycenaean Greek as it was spoken, but with a system that would best allow them to carry out their administrative recording tasks. It is difficult, therefore, to see a motivation within the context of the LB records for creating a sign * pye.

Conversely, potential motivations for inventing this sign as pte become clear when examining its corpus of attestations. The proportion of pte's attestations in Greek vocabulary words is remarkably high: it is found in seven nouns, appellatives, and adjectives, based on five Greek roots, which account for at least $54 \%$ of lexemes in which pte appears and over $90 \%$ of its total attestations. This includes terms in which the cluster /-pt-/ is formed by the addition of the agent suffix /-tēr/ to a labial root (1a-b), as well as those containing /-pt-/ in the root (2-5):

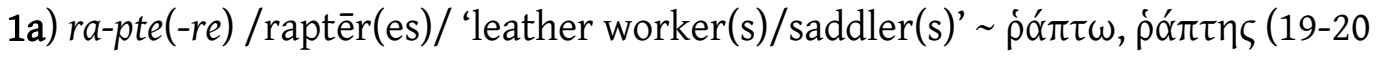
examples)

1b) ra-pte-ri-ja /raptēriai/, feminine plural adjective from ra-pte (1 example)

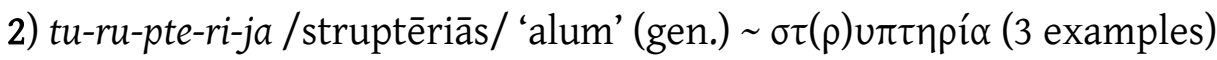

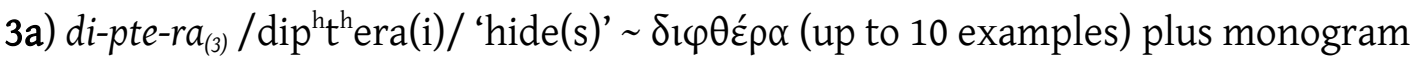
DI-PTE (1-2 examples)

3b) di-pte-ra-po-ro /dip $\mathrm{t}^{\mathrm{h}} \mathrm{erap}^{\mathrm{h}}$ oros/ (or /dip $\mathrm{t}^{\mathrm{h}}{ }^{\mathrm{h}}$ erapōlos/) 'carrier (or seller) of hides' (2-4 examples)

4) pte-re-wa /ptelewās/ 'elm' (gen.) $\pi \tau \varepsilon \lambda \varepsilon^{\prime} \bar{\alpha}$ (11-16 examples)

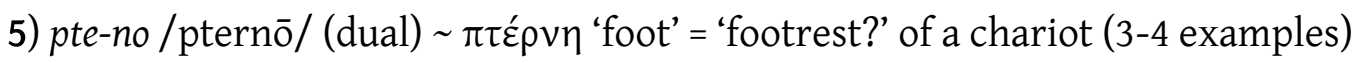


By contrast, pte appears in just 1-2 PNs (ka-pte, /Skaptēr/? or /Kamptēr/?; ptẹ-jo-kọ, obscure); two incomplete terms which may well be nouns (]pte-we, nominative plural noun, or dative singular noun or PN; ]-pte-si, probably a dative plural noun, perhaps ra]-pte-si? ${ }^{162}$; and 1-2 completely obscure terms, pte[ and ]pu-ptẹ[. These terms make up at most $46 \%$ of the lexemes in which pte appears, and just $7-9 \%$ of its total attestations. Considering that the majority of the LB corpus as a whole consists of proper nouns as opposed to vocabulary words, ${ }^{163}$ not to mention the high proportion of terms which are either non-Greek or at least not demonstrably Greek, these figures are very striking.

The proportion of pte's attestations in Greek vocabulary words is significantly higher than even those of the extra signs already shown to be of particular phonemic and/or morphological importance (e.g. $r a_{3}, 55 \%$ of certain attestations in Greek vocabulary words; $\left.a_{2}, c .50 \%\right)$ and comparable only to those attested almost entirely in a single term, e.g. dwe, c.80\% of whose attestations are in te-mi-dwe(-te, -ta). Note that several of the vocabulary words in which pte occurs are repeated throughout particular tablet series, ${ }^{164}$ a circumstance similar to that of te-mi-dwe-ta and o-da-twe-ta, which might particularly encourage the development of a more concise way of writing these terms. The evidence therefore suggests that pte was invented within LB due to its usefulness in representing a series of frequently-used Greek words, including those containing the productive suffix /-tēr/. Although it cannot be ruled out that *AB62 may simply not yet be attested, until or unless an example of this is produced by future discoveries it seems preferable to

\footnotetext{
${ }^{162}$ Alternatively, Melena 2014b:69 suggests $k u$-]pte-si, by comparison with the possible adjective ku-pe-te-jo (KN X 974.b), but without giving an interpretation.

${ }^{163}$ According to Bartoněk 2003:400, 73\% of lexemes in the LB corpus are onomastic in nature, and $27 \%$ are vocabulary terms.

${ }^{164}$ pte-re-wa/pe-te-re-wa recurs 11-16 times in the KN Se and So-series, and pte-no 3-4 times in the Sd-series; ra-pte(-re) appears 19-20 times in the PY An- and Ea-series, and di-pte-ra $a_{(3)} 8$ times on a single tablet, PY Ub 1318, as well as two other Ub- and Un-series examples.
} 
view pte as this sign's original value rather than *pye, a value primarily useful in expressing a morphological category which would rarely, if ever, actually appear in the written records.

\subsubsection{Summary of palatalised signs}

Since $\mathrm{ra}_{2}$ was inherited from LA, further similar signs could also have been inherited, though none would be likely to be nearly as frequent as $\mathrm{ra}_{2}$ and $\mathrm{ro}_{2}$; nor is it likely that if not inherited they would have been invented in $\mathrm{LB}$, since $\mathrm{ra}_{2}$ and $\mathrm{ro}_{2}$ are clearly the most morphologically useful in representing Greek $a$ - and o-stem endings. The situation of $t a_{2}$ is similar: while other LA signs could have existed and been inherited, the parallel of inherited $\mathrm{ra}_{2}$ and (probably) invented $\mathrm{ro}_{2}$ suggests the possibility of a sign for /tyo/ having been invented within LB, primarily for use in nouns, PNs, and adjectives with the common endings /-tios/ and /-tiōn/. Such a sign, whether inherited or invented, would be expected, like $\mathrm{ta}_{2}$, to have a high proportion of word-final occurrences; any further palatalised signs would be expected to be rare at best in word-initial position (though initial attestations in non-Greek words remain possible: cf. the [few] initial examples of AB76 and CH 069 [p.48, n.130], and AB66 [p.51, n.142] $)^{165}$.

The potential existence of signs for other palatalised consonants is more questionable. It is often assumed that the LB palatalised signs continue an LA palatalised consonant series, reflecting a systematic Minoan phonemic distinction; ${ }^{166}$ but the arguments put forward above (pp.44-6) against this assumption in the case of the labialised signs are equally valid for the palatalised signs, so that we cannot automatically expect the

\footnotetext{
${ }^{165}$ T. Meißner, pers. comm.

${ }^{166}$ E.g. L.R. Palmer 1955b:38 and 1963b:38-40; Davis 2014:193-6, 236-8; cf. EDG:xvi-xvii and Beekes 2014:4.
} 
existence of inherited palatalised signs in all, or even any, other consonant series. The evidence for Minoan palatalisation is in fact even slighter than that for labialisation: there was, at least, a whole LA series of consonants interpretable in Greek as labiovelars, whereas $r a_{2}$ and $t a_{2}$ are currently the only two known palatalised signs with LA antecedents. This does not, of course, exclude the possibility of further such signs existing; on current evidence, any such signs would be most likely to have an $a$-vocalism (but it is impossible to say whether the fact that $r a_{2}$ and $t a_{2}$ share this vocalism is linguistically significant, or merely the result of this being the most common vowel in both scripts). It will be more productive, however, to examine the prospects of any such signs being used or created within LB.

Signs for */dy/ and */ky/ would not be expected as these underwent palatalisation processes even in secondary contexts (e.g. */kak-yos-es/> ka-zo-e /katsohes/ kakíoveৎ); labiovelars probably would have done likewise, although the Mycenaean evidence is very uncertain. ${ }^{167}$ Palatalised signs in $j$ - and $z$ - are also not to be expected, since these would involve a sequence */yy/ (cf. p.44, n.120). There are no known Mycenaean examples of*/my/ or */ny/ (Heubeck 1979:242), but generally these both appear to have undergone the same treatment as */ry/ (Lejeune 1972f:155-6); the chances of a sign for either value having existed in LB do not, however, seem high given these sequences' rarity.

The existence of /wy/ clusters is in many cases difficult to determine since the sequence -wi-j-could represent either /wy/ or /wiy/; terms such as di-wi-jo/di-wi-ja and me-wi-jo, however, are shown by their alternate spellings $d i-u-j o / d i-u-j a$ and $m e-u-j o$ to contain

${ }^{167}$ For possible examples of $* / \mathrm{k}^{\mathrm{w}} \mathrm{y}-/$ and $* / \mathrm{g}^{\mathrm{w}} \mathrm{y}-/$, see Heubeck 1979:240-1. 
/wy/. ${ }^{168}$ It is therefore at least possible that other similar adjectival formations in -wi-jo/ -wi-ja underwent a similar resyllabification process to that seen seen with $\mathrm{ra}_{2}, \mathrm{ro}_{2}$, and $t \mathrm{a}_{2}$, becoming /-wyo-, -wya/, and hence signs for these values would be possibilities.

Similarly, although */sy/ > /-(y)y/ by assimilation (e.g. the o-stem genitive singular -o-jo $=/ \mathrm{o}(\mathrm{y}) \mathrm{yo} /<* /-\mathrm{osyo} /$ ) and $* / \mathrm{py} />/ \mathrm{pt} /(\mathrm{p} .55)$, secondary clusters of both types could have arisen by the same resyllabification process, and signs for /sya/, /syo/, /pya/ and /pyo/ might thus also be possibilities (although the sequences pi-jo and pi-ja are in practice much less common than si-jo/si-ja and wi-jo/wi-ja: DĀMOS gives up to 39 examples of pi-jo/-ja, 353 of si-jo/-ja, and 237 of wi-jo/-ja). All of these possible pyV, syV, and wyV signs would be likely to occur relatively frequently in word-final position in adjectives, nouns, and PNs, but rarely if ever word-initially. Any with values in - $a$, at least, could have been inherited from LA, though could equally have been LB inventions modelled on $\mathrm{ra}_{2}$ and $\mathrm{ta}_{2}$; any in -o might be more likely to be LB inventions, on the lines of $\mathrm{rO}_{2}$.

If the above explanation for the existence of pte is accepted, then it is possible that other similar $p t V$ signs, though probably not a complete series, might have been created on the model of pte (whether at a similarly early stage in the development of the script, or later on at a single site). Melena 2014b:70 suggests that pta would be likely, based on vowel frequency and its potential use in nominal endings (in /-ta/ or /-tās/), and that this

\footnotetext{
${ }^{168}$ Mycenaean */wy/ has been argued to have assimilated to / y)y/, since the feminine nominal/adjectival ending corresponding to the masculine eu-stems is always spelt -e-ja /-e(y)ya/, despite its presumed etymology */-ew-yh / (Heubeck 1979:241; Lejeune 1972f:171-3). However, this is the only possible evidence for this loss of /w/; the spellings of di-wi-jo/di-u-jo, etc., demonstrate that /wy/ was preserved in at least some cases (pace Heubeck 1979:241, n.10, and Meier-Brügger 1992:24), and the suggestion of Lejeune 1972f:173 that these examples of /wy/ are relatively recent developments seems implausible in the case of terms with such good IE etymologies as */diw-yo-/. It seems more likely that the -e-ja ending did not contain */w/, whatever its actual origin, which may even be non-IE (Meißner 2014; Meißner forthcoming gives a summary of the debate over the origins of the corresponding masculine nouns in /-eus/).
} 
could have been inherited from LA; although such an inheritance is of course possible, it is not necessary in order to explain pte (indeed, there are no other examples of new signs in -e based on inherited signs in $-a$ ). In fact, a survey of possible instances of /pt/ shows only a single known example of the sequence /pta/ in LB, in the TN a-pa-ta-wa /Aptarwa/ and the related ethnics a-pa-ta-wa-jo/-ja /Aptarwaios, -a/ "A $\pi \tau \alpha \rho \alpha /$ 'A $\pi \tau \varepsilon \rho \alpha)$. There are no examples of /ptu/ at all; /pti/ is perhaps found in the term pi-ti-ro ${ }_{2}$-we-sa if this is /ptillowessa/ (p.50, n.137) and certainly in the MN/ethnic $a_{3}$-ku-pi-ti-jo = /Aiguptios/. None of these is therefore a particularly likely candidate for the creation of a new sign; /pto/, however, is not only the most frequently-attested $p t V$ sequence after pte, but is similarly useful in both morphological and lexical terms, being found in

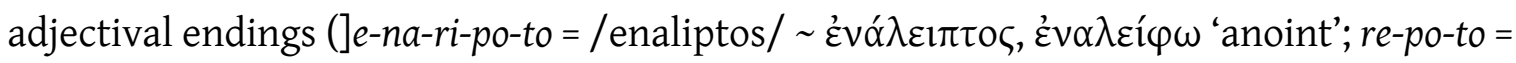

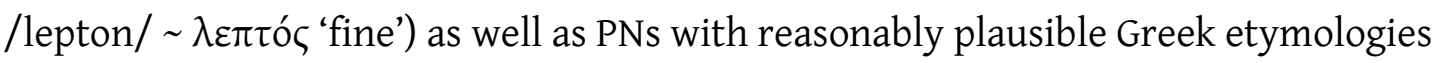

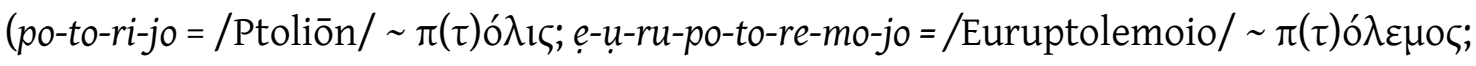

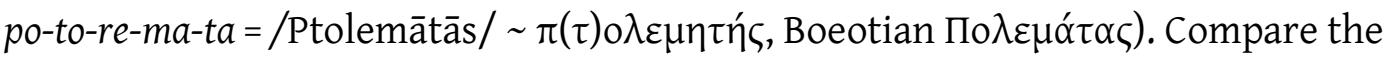
situation of the labialised dentals $d w e / d w o$ and twe/two, probably all created within LB and equally probably restricted to the most morphologically useful vowels $e$ and $o$. I thus regard pto as the only possible further $p t V$ sign which has a particularly high chance of existing.

Admitting the potential existence of signs which do not conform to the more usual $\mathrm{CV}$, $C y V$, or $\mathrm{CwV}$ structure might seem to raise the possibility that almost any consonant cluster could, in theory, be represented amongst the undeciphered signs. In practice, however, /pt/ and / $/ \mathrm{p}^{\mathrm{h}} \mathrm{h}$, as clusters of stops which can regularly appear as onsets as well as across syllable and morpheme boundaries, are fairly special cases: the only 
similar clusters are $/ \mathrm{kt} /$ and $/ \mathrm{k}^{\mathrm{h}} \mathrm{t}^{\mathrm{h}} /{ }^{169}$ If any other such extra-systemic signs were going to be invented, signs for $k t V$ might therefore be the most likely after $p t V$ - although the relatively high frequency of repeated terms such as ki-ti-me-na /ktimenā/ and ko-to-(i-)na /ktoinā/, particularly at Pylos where these are repeated throughout the E-series, might provide an argument against any such sign actually existing (given the repeated plene spelling of / kti-/ and / kto-/ in this context) as much as suggesting the possibility of such signs having been invented. In any case, a proposal to identify such a sign would need to justify its existence on the grounds of lexical and/or morphological utility as well as producing convincing readings.

\section{3: Conclusions}

Values of the undeciphered signs

This chapter's main purpose was to identify what types of sound-values are potentially most likely to exist amongst the undeciphered signs; the results can be summarised as follows:

\section{Core signs}

$j u$ is the most likely 'missing' core value to find, with zi and zu possibilities (but, contrary to usual assumptions, not ones that would necessarily have to exist). All of these would most probably be inherited from LA.

\footnotetext{
${ }^{169}$ Initial /bd-/ and /gd-/are found in classical Greek, but only very sporadically; /zb-/ is found only in terms of probable non-Greek origin (LSJ q.vv.)
} 


\section{Vowels}

$e_{2}$ and $o_{2}$, representing the diphthongs /ei/ and /oi/, would be the most likely values to find. The frequency of initial $e-u-$ and $o-u-$, compared to that of $a-u-$, makes signs for u-diphthongs unlikely; further aspirated vowels are similarly unlikely due to the observed spelling rules for hiatus, as well as their lower frequency than /ha/. Further signs of the $\mathrm{ra}_{3}$ type are also in practice unlikely.

\section{Plain consonants}

Further signs in the $p_{2}$-series, representing aspirated labials, are good probabilities, particularly if inherited from LA; other aspirated stops are probably not. There is no reason to expect the existence of any further signs representing voiced stops beyond the $d$-series.

\section{Labialised consonants}

Further signs in $n w$ - could well have been inherited from LA; the inheritance of further tw- or $d w$-signs cannot be entirely ruled out, but is judged to be improbable, as is their invention within LB. There is no good reason to assume a widespread inheritance of labialised signs from LA. Judging by the attestations of other $\mathrm{C}_{w}$-clusters, $r w$-and perhaps $k w$-signs might be the most likely candidates for invention in LB (with $e$-and/or $o$-vocalism), although $k w$ - might be represented by the $q$-series; $s w$ - signs are also possible, though less strongly motivated. 


\section{Palatalised consonants}

Further signs in the $r_{2}$ - and $t_{2}$-series could well have been inherited from LA, but the only likely candidate for creation within LB would be $t_{2}$. As for the labialised consonants, although further inherited examples cannot be ruled out, there are less strong grounds than is often assumed to propose a widespread inheritance of these signs from LA. The best candidates for creation within LB would be signs representing /wy/ or secondary /sy/, and perhaps secondary /py/.

\section{Others?}

If the explanation for the creation of pte given here is accepted, the possibility arises that other similar CC-signs might have been created; however, in practice the special status of the cluster /pt/ and the criterion of lexical and morphological utility means that potential values would probably be restricted to a corresponding pto, and possibly signs for the similar cluster /kt/.

Likewise, the argument that in the case of pte considerations relating to the structure of the script should be given less weight than those relating to a sign's potential usefulness in the context of the Mycenaean administrative records does not open the gates to identifications of signs representing any feature not currently known to be distinguished in LB. The criterion of usefulness would still tend to exclude signs representing, e.g., vowel length or /r/ vs. /l/: neither could contribute to greater efficiency of writing, nor is the lack of either likely to lead to serious ambiguity in a large number of particularly significant cases. The potential benefits of accuracy in representation offered by such signs would be outweighed by the drawbacks of the more complicated system that would be produced by creating them. 
This analysis has therefore provided a basis from which to approach the question of individual signs' potential values, allowing the theoretical plausibility of specific decipherment proposals to be evaluated, as well as helping to narrow down the range of probable values - for instance, if a sign's distribution suggests a vocalic value, these results show its likely values to be limited to two (/ei/ and /oi/) rather than the much larger number which might otherwise have been thought to be possible. However, this chapter has also gone beyond this primary function to make important contributions to the debate over the structure of LA and its use as evidence for Minoan phonology, as well as offering insights into the motivations behind the creation and use of extra signs within LB.

\section{$\underline{\text { LA and Minoan }}$}

This chapter has demonstrated that the origins of the LB palatalised and labialised signs are more varied than simply being inherited from, or modelled on, LA signs; thus, the hypothesis that palatalisation and labialisation were systematic phonemic distinctions in the Minoan language, while of course not disproved, is far less strongly supported than has often been assumed. It is also worth noting that a systematic distinction of labialisation and palatalisation at every possible point of articulation is typologically very unlikely: according to a survey of UPSID, almost two-thirds of languages which have phonemic labialisation have no more than three labialised phonemes, and the same is true of palatalisation, while only twelve known languages have both labialisation and palatalisation as phonemic features. ${ }^{170}$ The typological arguments which were used to argue for systematic labialisation and palatalisation in Minoan in fact, therefore, provide better support for the opposite conclusion, which is also more in accordance with the

\footnotetext{
${ }^{170}$ I am grateful to M. Valério for suggesting that I investigate this typological issue.
} 
palaeographic data - namely, that if Minoan had labialised and/or palatalised phonemes, these are likely to have been relatively few in number (with $/ \mathrm{k}^{\mathrm{w}} /, / \mathrm{n}^{\mathrm{w}} /, / \mathrm{r}^{\mathrm{y}} /$ and $/ \mathrm{t}^{\mathrm{y}} /$ being the most probable candidates).

This conclusion should lead to similar caution in approaching other hypotheses about Minoan based on the (assumed) structure of LA, which are often presented as being facts: for instance, that the lack of an LA correspondence for $a_{2}$ shows that Minoan had no /h/ phoneme; that the distribution of inherited signs amongst the five LB vowel series shows Minoan to have had a three-vowel system (p.8); and that the lack of a systematic distinction of voicing or aspiration in LB shows the same to have been the case in LA and thus proves Minoan to have had no phonemic distinction between these features. The important point demonstrated by this chapter is not that any of these hypotheses is any more or less likely than previously thought, but that we cannot simply assume that apparently 'odd' features of LB map straightforwardly on to features of LA, nor that any such LA features are themselves straightforward reflections of linguistic features of Minoan: processes of script creation and use are varied and complex in their relationship to the language represented even within a single script (see further below), let alone in the creation of a daughter script from its parent. It is only through analysis of the processes involved in the creation and subsequent use of LB that we can hope to be able to work backwards from this to even a partial understanding of the LA script and perhaps, through this, the Minoan language.

\section{$\underline{\text { LB inventions and use of signs }}$}

This chapter has explored the wide range of motivations which may lie behind the use of the LB extra signs, especially those which were created within LB. In particular, a 
recurring theme has been the creation of signs, not at random or in order merely to 'fill up' a series of signs, but in order to fulfil specific useful functions, whether this is the more accurate and/or efficient representation of frequently-repeated terms ( $d w e, d w o$, twe, two, pte) or that of particularly important grammatical categories $\left(a_{2}\right.$ for neuter plurals, $r a_{3}$ for $a$-stem plurals; cf. the emphasis on the representation of dative plurals over singulars in the spelling conventions for $i$-diphthongs) or morphological formations ( $d w e, d w o$, twe for the suffixes /-wos-/ and /-went-/; $r o_{2}$, like the inherited $r a_{2}$ and $t a_{2}$, for adjectives in /-ios, -ia/). of course, as shown by the labialised dentals in particular, various different motivations may have interacted to influence the original creation and/or the continuing use (or not) of a particular sign. This investigation has, therefore, not just been of use in helping to determine probable sound-values for the undeciphered signs: it demonstrates the complex interplay of different and potentially competing factors (such as phonemic accuracy, efficiency in writing, and economy of the overall system) which operate on the creation and development of any writing system - and, in addition, the importance of the context in which the writing system is used. The newlycreated extra signs of LB were not created in order to represent the most important phonological features, grammatical categories, or morphological formations of Mycenaean Greek per se, but those which were felt by the users of the script to be the most useful in the context of their written documents. 


\section{Chapter 2: The undeciphered signs - individual studies}

This chapter presents individual studies of each of the 14 undeciphered signs, in numerical order for ease of reference. Each study first considers the attestations and palaeography of the sign in question: the sign's palaeographic variation between and within sites is analysed and particularly problematic or controversial examples discussed (for full details of all possible attestations of each sign, the reader is referred to the relevant section of the accompanying 'Undeciphered signs' spreadsheet). ${ }^{171}$ The sign's prospects of decipherment are then reviewed: evidence from its distribution, possible spelling variations, and/or proposed identifications of Greek words are discussed in comparison with the results from Chapter 1 as to the relative probability of different types of sound-values. Although full references to all previous decipherment attempts will be given, due to considerations of space detailed discussion will be limited to those proposals which either stand the greatest chance of being correct (i.e. which may be supported by at least one, and preferably several, of the types of evidence listed above) and/or are currently widely accepted. It should be stated here that the aim of this chapter is not to propose a full decipherment of any or all of the undeciphered signs. Rather, it is to establish what currently can and cannot be relatively securely established about each sign - whether this is that the sign in question is likely to have a specific sound-value or a particular type of value, or only that there is currently insufficient evidence even to suggest a tentative sound-value - in order to provide the best possible basis for future decipherment attempts in the light of any further evidence which may someday be found.

\footnotetext{
${ }^{171}$ Note that in general only examples classed as 'certain' or 'possible' (underdotted in the spreadsheet) will be included in the discussion here, with the exception of cases where examples classed as 'doubtful' (underdotted and italicised in the spreadsheet) would, if correctly identified as the undeciphered sign in question, be of particular importance for its palaeography, distribution, and/or corpus of attestations (e.g. in the case of signs with very few certain examples).
} 
Before beginning these individual studies, however, it is first necessary to consider the status of this whole group of signs within the LB script. The relatively frequent claim that some of the undeciphered signs may represent specifically non-Greek/Minoan phonemes implies that these signs, at least, are thought to have a special status within the syllabary, different from that of the 'normal' signs representing Greek phonemes; such 'Minoan' signs tend to be identified based on their attestations only in non-Greek words and/or solely or primarily at Knossos. ${ }^{172}$ It is certainly possible that some of the undeciphered signs may have values whose use in representing Greek phonological features was marginal: the presence of nwa amongst the extra signs shows that such signs could exist (p.43), and as already discussed, this might be the main context of use for other signs in $n w^{-}$, or signs for zi and perhaps $z u$ (pp.12-13).

None of these possibilities, however, involves sound-values that are non-phonemic in Greek; rather, they involve sequences of phonemes which are, for various reasons, likely to have been rare or non-existent in Mycenaean Greek. Moreover, the fact that nwa is widely attested across multiple sites on Crete and the mainland shows that distribution is not a reliable criterion for establishing this kind of 'marginal' status in Greek phonological terms, ${ }^{173}$ especially when compared to the much more restricted distribution of some other signs whose sound-values are clearly useful in Greek (e.g. twe, $\left.r a_{3}\right)$. The criterion of attestation only within non-Greek words - or, strictly speaking, words not (currently) identifiable as Greek - is even more problematic: since a large proportion of LB terms have this status, rarely-attested signs may well appear only in

\footnotetext{
${ }^{172}$ E.g. Owens 1991-1993:265 (*49); Melena 1987b:223-4 ( $\left.{ }^{*} 22,{ }^{*} 56\right)$ and forthcoming:4, 21, $25\left({ }^{*} 18,{ }^{*} 47,{ }^{*} 49\right)$; Palaima-Sikkenga 1999:602-3 $\left({ }^{*} 22,{ }^{*} 56\right)$.

${ }^{173}$ The possibility that chance is responsible for a given sign's absence from some sites makes this even less reliable: it may not be coincidental that ${ }^{*} 49$ - the only undeciphered sign now certainly found only at Knossos - is also one of the rarest of these signs (see pp.106-7).
} 
such words, and moreover it is impossible to tell how far our inability to identify these terms as Greek is due precisely to the fact that they contain undeciphered signs.

Of course, it remains a possibility that some LA signs were retained, particularly in Cretan LB, in order to represent non-Greek phonemes in Minoan PNs, TNs, and/or loanwords; but as remarked above (p.7), the chances of deciphering any such signs are close to non-existent. This possibility will be discussed when relevant, but in general, unless strong evidence is found to the contrary, the undeciphered signs will be treated here as regular components of the LB syllabary, differing from the other core or extra signs only in the chances of attestation which have led to their current 'undeciphered' status.

\section{$\underline{2.1: * 18} \stackrel{\text { "II }}{1}$}

*18 is a rare sign, with 7-11 examples at Knossos and possibly Mycenae. Its basic form is a 'head' with a horizontal and a vertical stalk below, with 'horn'-like elements on top of the head: ${ }^{174}$ its variants can be divided into two main types. Type 1 forms have a round head with 2-4 separate 'horns'; this includes a more elaborate version, Type 1a (H117; all majuscule) and a simpler version, 1b, with two or three 'horns' (H103, H118, H132, and the probable minuscule example by H117 on Da 1098 [[lat. inf.]]). Type 2 has a more triangular head with a single curved stroke forming the top and 'horns' ${ }^{175}$

\footnotetext{
${ }^{174}$ The suggestion that ${ }^{*} 18$ is a variant of ${ }^{*} 17=z a$ (Georgiev 1956:80) is shown to be incorrect by H103, H117, and H118's consistent differentiation of the two via the presence or absence of these 'horns'.

${ }^{175}$ Although both the examples of this type, on KN X 7739.b and 7944, are read as uncertain by COMIK and $K T^{5}$, I follow Melena forthcoming:3 in regarding these as extremely probable, if not certain, examples of *18.
} 

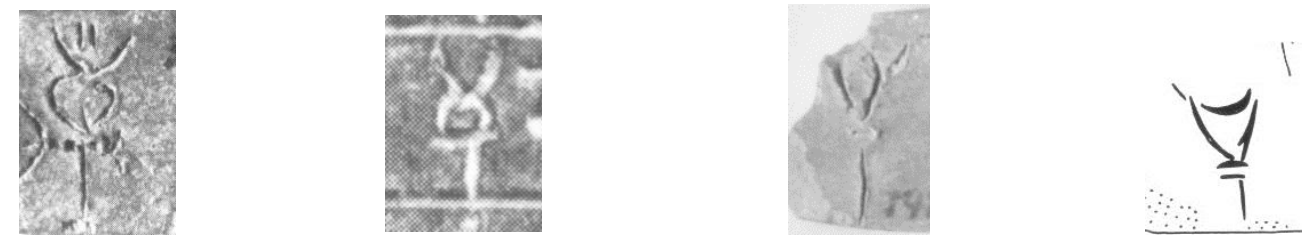

Type 1a: ${ }^{.16}$ Db 1211.B (H117) 1b: Ap 639.4 (H103), Dl(1) 950 (H118) Type 2: X $7739(-)^{177}$

H117 frequently alternates between more complex majuscule and simplified minuscule variants; ${ }^{178}$ however, Type $1 \mathrm{~b}$ is not merely a minuscule variant, since H118's example is majuscule. Type 2 seems likely to have developed through further simplification from a Type 1 form similar to that of H103 (with two 'horns' positioned on top of the head), since the same basic features are present, but require fewer strokes.

The only possible mainland example of ${ }^{*} 18$ is on MY Ge 603.1 (right), ${ }^{179}$ in the Image sequence $d a-r a-[]-.m i-t a-q e,{ }^{180}$ which is usually interpreted as two terms for removed for herbs or spices, da-ra-[.] and mi-ta-qe /mint ${ }^{\mathrm{h}} \overline{\mathrm{a}}-\mathrm{k}^{\mathrm{w}} \mathrm{e} /$ 'X and mint' (Docs ${ }^{2}: 225-30$; copyright DMic q.vv). From autopsy, TITHEMY's suggested reading da-ra-*18 (with a Type $2{ }^{*} 18$ simplified still further to give a flat head with no 'horns') is certainly possible, though so is -zạ (with an unusual triangular head: MT II), and a rather atypical -jọ (SCM;

\footnotetext{
${ }^{176}$ Images: COMIK, except 950 (MER).

${ }^{177}$ It is impossible to say whether this and 7944 are by the same hand. The form on $\mathbf{7 7 3 9}$ has two horizontals at the base of the head, while that on $\mathbf{7 9 4 4}$ has only one, but without further Type 2 examples it is uncertain whether this is diagnostic. Melena forthcoming:3 describes these two examples as 'in H118 style' and even suggests (n.5) that they were written by H118; in terms of the sign's formation, however, H118's example is much closer to Type 1.

${ }^{178} \mathrm{Cf}$. Bennett 1966:297. For the embellishment at the side, cf. (e.g.) H117's $z a$ and $p u_{2}$, or $i$ in H104 and on TH Z 866-8.

${ }^{179}$ Photo: author (NAM). Meriggi 1955:70 identified an example of *18 on MY Oe 122 but this is an example of $z a$ with an embellishment at the side, as frequently seen at Knossos (n.178, above): SCM; TITHEMY.

${ }^{180}$ I follow SCM in reading the -mi- as certain; TITHEMY has - mi i-.
} 
MT IV) cannot be entirely excluded. ${ }^{181}$ The condition of the tablet makes it impossible to give a more definite reading.

Although *18 has no generally accepted LA correspondence, both A333 and A347 are reasonably close in form to *18 (Types $1 \mathrm{~b}$ and $1 \mathrm{a}$, respectively), and are therefore candidates for *AB18 (it is also possible that the two could in fact be variants of the same LA sign). ${ }^{182}$

\section{Images removed for copyright}

reasons

\section{A333: HT Wc 3009 A347: KH 6.3}

It is thus quite plausible that ${ }^{*} 18$ was inherited from LA (particularly given that it is used almost entirely in PNs, many or all of which may be non-Greek in origin), but this remains uncertain until a single antecedent can be more definitely identified.

\section{Prospects of decipherment}

Since ${ }^{*} 18$ has no more than 11 examples, all its certain examples are (probably) in PNs, ${ }^{183}$ with no plausible examples of spelling alternations for any of the longer PNs, ${ }^{184}$ and its

\footnotetext{
${ }^{181}$ None of the other suggestions (-qa [MT II]; z 1 [MT IV]; or that this sign is in fact just a trace of the erasure [TITHEMY]) are palaeographically plausible.

${ }^{182}$ Faure 1979:131, n.1 [L174 = A347]; Melena 2014b:8 (cf. Melena 2009 [unpublished]:165). Images: GORILA.

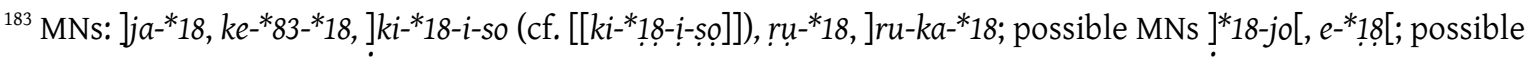
PN ]di-*18 (the last two suggested by Melena forthcoming:3). *18-to-no is probably a WN (nom.) rather than an MN (gen.): see Killen 1966:210-2 on the probability that the sequence *18-to-no , / tu , MUL 2 (KN Ap 639.4) is '*18-to-no and her daughter' rather than 'the daughters of *18-to-no'.

${ }^{184}$ The only possibility would be ]ru-ka-*18 po-ru-ka-to (MN, KN Vc(1) 74), which is both based on a restoration and without contextual support (the former is a 'shepherd' in the J1 sheep department while the latter is listed without context in the RCT).
} 
single possible example in a vocabulary word (da-ra-*18) remains both uncertain and obscure ${ }^{185}$ its decipherment prospects are poor. Its distribution (initial: 1x, medial: 1-2x, final: 4-6x, uncertain: $2 \mathrm{x})$ makes it likely to be a $C(C) V$ sign. The term $k i-{ }^{*} 18-i-$ so may well contain a plene spelling of an $-i$ diphthong, implying that ${ }^{*} 18$ 's vocalic value is more likely to be $a, e$, or o than $i$ or $u,{ }^{186}$ if this is the case, then ${ }^{*} 18$ would be an extra rather than a core sign ${ }^{187}$ Bearing in mind that ${ }^{*} 18$ may well have been inherited from LA (NB that its distribution is quite unlike those of the 'extra' signs invented in LB, as discussed in Chapter 1), the most plausible values would be $\mathrm{pe}_{2},{ }^{188} \mathrm{po}_{2},{ }^{189} \mathrm{re}_{2}{ }^{190} \mathrm{te}_{2}, \mathrm{to}_{2},{ }^{191} \mathrm{nwe}$, or $\mathrm{nwo}$, though other palatalised or labialised values might also be possible, especially with a vocalic value in $-a .{ }^{*} 18$ 's reasonably high proportion of word-final attestations would fit well with an $r_{2}$ - or $t_{2}$ - value. Its single initial attestation might make such a palatalised value less likely, though not impossible since this may be a non-Greek name (note the initial attestations of these signs' LA and CH correspondences: p.48, n.130 and p.51,

\footnotetext{
${ }^{185}$ Contextually, this should be a herb or spice; however, the suggestions of */dragwāa $~ \sim \delta \rho \alpha \dot{\beta} \eta$ 'Arabian

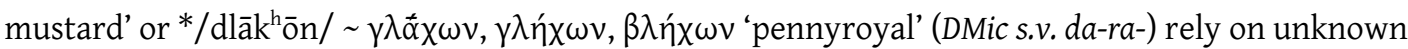
etymologies (CDE) and, in the latter case, a three-way alternation of /dl-/ /gl-/ /bl-/; moreover they do not produce plausible values for $* 18$, signs for voiced labiovelars or aspirated velars being improbable (p.33).

${ }^{186} \mathrm{~A}$ hiatus cannot be entirely excluded, but a sequence /ihi/ or /uhi/ is still relatively unlikely: there is only one known example of the sequence /-ihi-/ in the LB corpus (o-pi-i-ja-pi = /opi-hiāph ${ }^{\mathrm{h}} \mathrm{i} /$, KN Sd-series), and only two certain instances of - $u-i$ written without the expected glide, both obscure (]ku-i-so, KN Da 5214; pu-i-re-wi, MY Go 610.4): see Meißner 2008:508-10.

${ }^{187}$ Godart et al. 1990-1991:375-6 argue that *18 is more probably a complex sign (representing a disyllable) than a doublet, given its appearance in the (probably complete) MN $]^{*} 18-j o[$ : disyllabic words in -jo are very rare in the LB corpus (7 examples out of c.500 terms in -jo). However, complex signs arguably represent a monosyllabic form of the disyllabic sequence they correspond to (e.g. $r$-jo $=/ \mathrm{r}(\mathrm{i}) \mathrm{yo} /, \mathrm{ro}_{2}=/ \mathrm{ryo} /$ : Lejeune 1972b:100-1); in any case this term is not certainly complete.

${ }^{188} \mathrm{~A}$ plausible candidate for $\mathrm{pa}_{3}$ already exists in sign ${ }_{56}$ : see p. $115 \mathrm{ff}$.

${ }^{189}$ Considered but rejected by Melena forthcoming:4, on the grounds that a series originating in Minoan ought to have only three vowels; but cf. p.8.

${ }^{190}$ Suggested by Melena 1974a, but this was on the basis of a reading of Da 1098 lat.inf. (clearly referring to the same person as ]ki-*18-i-șo on Da 1363) as [[ki-rẹ-ị-so]]; Killen 1975 showed that the former more probably reads [[ki-*18-i-sọ]]. ${ }^{191}$ Proposed by Melena 1985:483, 2014b:82, and forthcoming:3-4.
} 
n.142); nwa is likewise never found initially, as expected in Greek (p.43), but it cannot be ruled out that LB might record a non-Greek name in /Nw-/. Although a $p_{2}$-value might therefore appear to best fit the sign's distribution, the evidence of only c.10 examples is far from conclusive on this front.

On the basis that there are several MNs ending in $-^{*} 18$, Melena 1985:483 and forthcoming:3-4 concludes that *18 is most likely to have an o-vocalism. This, however, assumes these to be Greek MNs in /-os/ or /-ōn/; given the high proportion of nonGreek names at Knossos (particularly amongst relatively low-status groups such as the 'shepherds' of the D-series, which most of these MNs are), ${ }^{192}$ it is probable that most if not all are of non-Greek origin, and there is no reason to suppose them all to have been adapted to Greek morphology. Attempts to identify this sign's value more precisely through assigning Greek etymologies to these names (as Melena does in arguing for the value $\mathrm{to}_{2}$, as well as in considering, but rejecting, $\mathrm{po}_{2}$ ) are therefore highly insecure, and the current state of the evidence does not permit anything more than a highly tentative assessment of this sign's possible values. Note also that if any of the undeciphered signs do specifically represent Minoan phonemes, ${ }^{* 18}$ (as a sign only certainly found at Knossos) may be one of the more likely candidates for this status; ${ }^{193}$ evidently, however, this would depend on establishing that it was in fact inherited from LA, and with no more than 11 examples (including the possible example from Mycenae) this sign's lack of certain examples elsewhere may well be due to chance.

\footnotetext{
${ }^{192}$ See, e.g., Baumbach 1986, 1987, and 1992; Ilievski 1992; Firth 1992-1993:95; Morpurgo Davies 1999:400-1.

${ }^{193}$ Cf. Melena forthcoming:4.
} 


\section{$\underline{2.2 * 19}$ 只}

This sign has no more than 8 examples from Knossos, Pylos, and Thebes, all but two of which are uncertain or controversial. The two unproblematic examples of * 19 have the same basic structure, with a round 'head' and horizontal cross-bar similar to za or *18, but with a triangular or rectangular 'body', closed at the bottom by a horizontal, instead of a vertical stalk. ${ }^{194}$

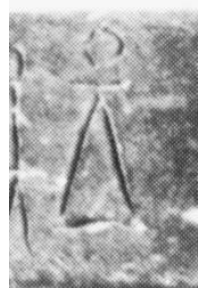

KN Ag 90 (H124-A)

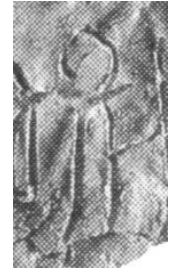

TH Wu 430. $(-)^{195}$

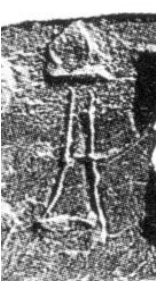

The uncertain * 19 on KN X 9014 (left) ${ }^{196}$ has also been suggested to be dwe (COMIK), presumably based on the presence of the line bisecting the 'body': made up of two strokes, this could be an even more simplified version of the 'arms' of dwe as seen on KN So 894. However, this form of 'head' is unparalleled in $d w e$, and the overall structure is very similar to the Theban *19; I therefore regard this as a very probable example of ${ }^{*} 19$.

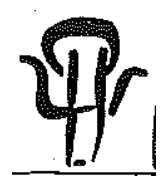

dwe: KN So(1) 4449 (H130)
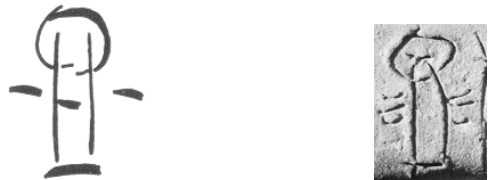

So $894.3(-) \quad$ PY Sa $791(\mathrm{H} 26)^{197}$

\footnotetext{
${ }^{194}$ This distinctive difference means that the suggestion that ${ }^{*} 19$ is a variant of $z a$ has no more support than the similar suggestion for *18 (Georgiev 1956:80; see p.71, n.174).

${ }^{195}$ Photos: MER; TFC 3 .

${ }^{196}$ Photo: MER.

${ }^{197}$ Drawings: COMIK; photo: MER.
} 
The remaining five possible examples, all at Pylos, are more controversial. They can be divided into three groups:

1) pi-*19-te (PY An 427.3), e-pi-*19-ta (Vn 10.2.5): all attributed to H3 (but see p.78).

\section{Images removed for copyright reasons}

\section{$\begin{array}{lll}\text { PY An } 427.3 & \text { Vn } 10.2 & \text { Vn } 10.5^{198}\end{array}$}

The reading of these three signs as ${ }^{*} 19$ is now fairly widely accepted, ${ }^{199}$ mainly due to the attestation of $e-p i-{ }^{*} 19-t a$ at Thebes. Previous arguments for reading them as $p u$ relied on the presence of the middle vertical and, on Vn 10.2.5, three small 'feet' as opposed to the single horizontal base of An 427.3 and all other examples of *19 (Palaima 1980). However, the structures of these signs, with a cross-bar and separate, almost circular 'head', are quite different from that of $\mathrm{pu}$, whose left-hand 'leg' typically extends up and around to form the top of the sign; even the example on An 424.1 (also by H3), whose top curves back on itself to an unusual extent, shows this distinct difference, and cf. this hand's more typical example on An 427.3.

\section{Images removed for copyright reasons}

\section{An $427.3 \quad$ An $424.1^{200}$}

\footnotetext{
${ }^{198}$ Photos: author (NAM).

${ }^{199}$ E.g. PoNIV reads $* 19$ as certain in all three cases. See also Aravantinos et al. 2006; Civitillo 2008.

${ }^{200}$ Photos: author (NAM).
} 
Palaima 1980:198 ascribed these differences to influence on the sign's form from the preceding $p i$; however, this cannot fully explain such an unusual structure for $p u$, nor is it easy to explain the same 'accidental' modification of a sign-form occurring consistently on three separate occasions, even with the presence of pi each time. The same objection applies to the suggestion that the addition of a middle leg to the usual form of ${ }^{*} 19$ could be due to this same process of influence from pi (Civitillo 2008:132); but there are too few extant examples of $* 19$ to entirely rule out the possibility of this being simply a variant form of the sign. Ultimately, even without the support of e-pi-*19-ta at Thebes, identifying these forms as *19 rather than pu requires the assumption of much less structural modification of the sign; ${ }^{201}$ I therefore follow PoN IV in reading e-pi-*19-ta and $p i^{*}{ }^{*} 19-t e$. Note that the attribution of $\mathrm{Vn} 10$ to $\mathrm{H} 3$ is mainly based on this sign (Palaima 1988b:70); it is difficult to say whether the slight difference between the forms of * 19 on these two tablets - mainly in the presence of 'feet' vs. a horizontal base - are significant. The same applies to the other slight differences observed during autopsy between forms on Vn 10 and those on H3's other tablets, An 427 and 424 (most notably in the form of $k o$ ). I therefore prefer to attribute $\mathrm{Vn} 10$ only to 'H3?'.

2) ti-ri-*19 (PY Jn 725.3) $)^{202}$ is uncertain due to a break in the tablet: all that is Image preserved is the top of a sign, consisting of a roundish shape with a separate removed for copyright horizontal below. *19 is by far the most probable reading; although due to the reasons damage za cannot be entirely excluded, the rounded shape of the head for

\footnotetext{
${ }^{201}$ Although pu might appear a better reading from an interpretative point of view, allowing the interpretation of e-pi-pu-ta as /epiph ${ }^{\text {hta/ }}$ 'saplings' (Docs':349-50; Palaima 1980:200-1), this still produces difficulties of interpretation in the context of Vn 10: see p.81, n.211. ${ }^{202}$ Photo: author (NAM).
} 
this sign is paralleled only at Knossos (Driessen 2000:pl.42) and not seen in other examples of za by $\mathrm{H} 2$.

3) $p u_{2}{ }^{*} 34-{ }^{*}+9(\mathrm{Wr} \text { 1374. } \gamma, \text { Ciii })^{203}$

Image This reading appears much less plausible than the alternative -za removed

for since, although this precise form is not paralleled in either sign, both

copyright the oval head and the second oval shape below the cross-bar are reasons better paralleled in za than *19 (for the former, cf. KN Xd 111.b, H124-R, above right $)^{204}$.

The corpus of *19 therefore contains seven certain or probable examples from Knossos, Thebes, and Pylos, as well as one more doubtful Pylian example. *19 has no known LA antecedent, but this may well be due to chances of attestation given the rarity of * 19 and the fact that $\mathrm{CH} 044$ is seems a fairly probable correspondence: note the similarity of some of its examples (below) to the Knossian and Theban forms of *19 in particular. ${ }^{205}$

\section{Imageremoved for copyright reasons}

However, ${ }^{*} 19$ 's wide, albeit infrequent, distribution on the mainland as well as Crete (including its attestation in at least one vocabulary word of Greek appearance: see

\footnotetext{
${ }^{203}$ Photo: author (NAM).

${ }^{204}$ Image: Driessen 2000:pl.11.

${ }^{205}$ Drawings: CHIC.
} 
below) suggest that it was used to write a syllable found in Greek, rather than only being retained to represent a Minoan phoneme.

\section{Prospects of decipherment}

The distribution of *19 (all examples in word-medial or final position) suggests a $C(C) V$ sign, but offers little other evidence as to its value (the fact that this sign follows $-i$ in almost all of the terms in which it is [probably] attested - e-ri-*19, e-pi-*19-ta, pi-*19-te, and ti-ri-*19- is probably only due to chance and the very small sample size). ${ }^{206}$

As the only vocabulary word in which ${ }^{*} 19$ is certainly attested, ${ }^{207}$ e-pi- ${ }^{*} 19$-ta therefore in principle offers the best evidence for a possible decipherment. ${ }^{208}$ At Pylos, it refers to wooden items used in making wheels/chariots, listed on Vn 10 as contributed by the du-ru-to-mo (/drutomoi/ 'wood-cutters' or 'carpenters') and the ro-u-si-jo a-ko-ro (/Lousios agros/, 'the Lousian territory', a place in the Hither Province) to the a-mo-te-jona(-de) (/(h)armoteiōna(-de)/: 'wheel-wright's/chariot-maker's workshop') alongside equal numbers of a-ko-so-ne (/aksones/, 'axles'). ${ }^{209} \mathrm{TH}$ Wu 430 presumably records a delivery of the same items to Thebes, although no further contextual information is

\footnotetext{
${ }^{206}$ Such a distribution might suggest either a consonantal value in $j$ - (frequently used to represent a glide after $/ i$ ) or a vocalic value in $-i$ (with the appearance of $-i$ in the preceding syllables being largely due to the writing of dummy vowels in consonant clusters). However, the only missing value to be expected in the $j$-series, $j u$, is very probably supplied by ${ }^{*} 65$ (p.11 and p.139ff), while in most instances -Ci- ${ }^{*} 19$ - is unlikely to represent /CCi/ (e-ri-*19 and ti-ri-*19 because a syllable-final /r/ followed by a consonant would not usually be written; e-pi-*19-ta because this is usually, and plausibly, interpreted as a compound in /epi-/).

${ }^{207} \mathrm{pu}_{2}{ }^{*} 34-{ }^{*} 19$ will be excluded from the discussion here since not only is it not a probable example, but any interpretation of this term also relies on interpreting *34; in any case there is no proof of the usual assumption that this is a neuter plural adjective describing TELA+PU (which appears on Wr 1374. $\boldsymbol{\alpha}$ ).

${ }^{208}$ Decipherment attempts dating from before this term was recognised (e.g. Melena 1985:486, n.26: nwo; Witczak 1992a: twi) rely on a single certain attestation, the MN e-ri-*19, and are therefore too insecure to be taken into account here.

${ }^{209}$ Docs $^{2}$ :349-50; Plath 1994:64-5; DMic q.vv.
} 
available. ${ }^{210}$ It is, however, difficult to establish precisely what part of a wheel or chariot this term could refer to, since the only clue is the $1: 1$ ratio of $e-p i^{*} 19-$ ta to axles (i.e. one per chariot); possible chariot components might include the pole or pole-stay, or some part of the chariot body; ${ }^{211}$ wheel components are presumably less likely, since two would be required per chariot. ${ }^{212}$ If the term is correctly interpreted as a compound in /epi-/, its root consists of only a single syllable (-*19-t-), making its linguistic identification equally difficult unless a spelling alternation or later Greek correspondence is found.

The only possible spelling alternation for this term in LB is e-pi-zo-ta, ${ }^{213}$ found on two sword tablets from Knossos ( $\operatorname{Ra}(2) 984.2$ and 1028.B, H127) as well as on $\mathrm{HV} \mathrm{Rb} 1 r$. and $v$. (this tablet lacks ideograms, but is classed as a weapons tablet based on this term). ${ }^{214}$ The various proposals to identify e-pi-zo-ta and e-pi-*19-ta as the same term, however, all involve assuming a term with a very vague meaning such as 'covering' with two different technical applications, that of a scabbard (or a hilt-wrapping) for swords and

\footnotetext{
${ }^{210}$ Nothing else is written on the sealing, which was found in the 'Treasure Chamber' along with two others recording animals/animal products (Wu 429: ${ }^{*} 180+D I$, possibly an animal hide: Vandenabeele-Olivier 1979:277; $\mathrm{Wu} 431: \mathrm{CAP}^{\mathrm{m}}$ ) and a single tablet (Ug 432) recording quantities of an unspecified liquid; there is therefore no evidence for the texts from this room forming a coherent grouping in the way that the Odos Oidipodos sealings do (Piteros et al. 1990; Killen 1992).

${ }^{211}$ The reading e-pi-pu-ta /epiph ${ }^{\mathrm{h}}$ ta/ has been suggested to refer to saplings used to form the 'arcades' between the chariot pole and the pole-stay (Palaima 1980:200-1). It is, however, unclear why these should be recorded as the raw material 'saplings' in contrast to the axles: are the du-ru-to-mo supplying one finished product (axles) and one raw material (saplings) or are two sets of raw material being differently recorded as 'saplings' and '[for making] axles'?

${ }^{212}$ On the construction of Mycenaean chariots, see Åkerström 1978; Crouwel 1981 (esp. Glossary, pp.23-4; Ch.V, 'Chariots, Structural Development'; and Fig. 7, p.116). The LB evidence for chariot terminology is surveyed, and compared to Homeric terminology, by Plath 1994 (who shows that for the most part the two do not overlap: pp.397-9), while Vandenabeele-Olivier 1979:76-139 gives a survey of chariot ideograms.

${ }^{213}$ Aravantinos et al. 2006:6; Civitillo 2008:136ff.

${ }^{214}$ Aravantinos-Vasilogamvrou 2012:46.
} 
that of a protective axle casing for chariots, ${ }^{215}$ not to mention that the only evidence for the existence of such axle casings appears to be these etymological interpretations. ${ }^{216}$ Moreover, most proposals to read the sign in this way fail to account for the existence of a doublet of zo; the only one to assign it a specific value is Duhoux 2008:349, who suggests that ${ }^{*} 19$, as $z_{2}$, represents specifically /tso/ (where zo could be /tso/ or /dzo/: on the zseries, see p.11, n.22). As Chapter 1 showed, however, there is no good reason to expect signs distinguishing voiced stops in any consonant series apart from the $d$-series (p.33), and the same would seem to apply to those distinguishing voiceless stops. Given their very different contexts of attestation and the fact that the apparent similarity between these terms is likely to be due only to their happening to share the prefix /epi-/ and a neuter plural ending in /-ta/, it is far more probable that e-pi-zo-ta and e-pi-*19-ta are simply unrelated words.

A second possible spelling alternation for ${ }^{*} 19$ concerns the TN pi-*19-te: Melena forthcoming:7-8 compares this to another Pylian TN, pi-ru-te, and therefore suggests the

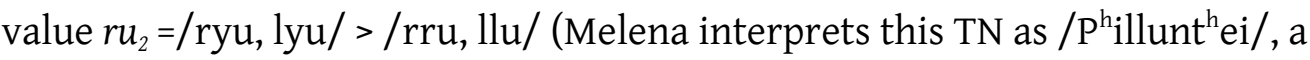
metathesised form from */Ph $u$ llint ${ }^{\mathrm{h}} \mathrm{os} / \sim \varphi v \lambda i^{\prime} \alpha$, but the precise interpretation is of little importance if the alternation is shown to be contextually secure). Further $r_{2}$-signs were argued in Chapter 1 to be fairly plausible as inherited signs (p.59), which would fit with

\footnotetext{
${ }^{215}$ Aravantinos et al. 2006:6 interpret these as /epidzōsta/, a verbal adjective related to $\dot{\pi} \iota \zeta \dot{\omega} v v v \mu$ (cf.

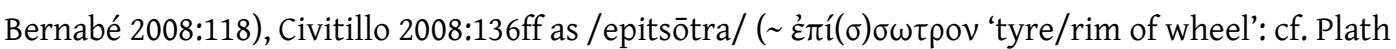
1994:332-3). The first is difficult since the root $\zeta \omega \sigma$ - in classical Greek generally refers to fastening (clothes or weapons) specifically on one's body, and it therefore does not seem particularly likely that a related verbal adjective could have meant simply 'fastened on' vel sim.; the second involves assuming that $\dot{\varepsilon} \pi \hat{i}(\sigma) \sigma \omega \tau \rho o v$, a technical term in Homer for the rim of a wheel, could have had a more general meaning of 'covering' applicable also to swords. Duhoux 2008:349, comparing e-pi-*19-ta only to $\dot{\pi} \pi \dot{i}(\sigma) \sigma \omega \tau \rho \circ \mathrm{v}$, suggests that the former refer to 'pieces of wood...long and large enough for two tyres'; but it hardly seems plausible that a delivery of 50 pieces of wood intended to yield 100 tyres would be recorded as 'tyres: 50 '. ${ }^{216}$ No mention of such casings is made in Crouwel 1981:78-81.
} 
the suggestion above that ${ }^{*} 19$ may at least have an attested $\mathrm{CH}$ correspondence; a palatalised value would also fit with the sign's distribution, since (like the known palatalised signs) it is never attested word-initially, although the sample size is so small that this is hardly conclusive. There is, however, no contextual reason to identify pi-*${ }^{*} 19-$ te with pi-ru-te: they are attested alongside entirely different sets of TNs and in completely different contexts, ${ }^{217}$ and the most that can be said is that there is some slight evidence to suggest that both may be in the Hither Province. ${ }^{218}$ There is no particularly good reason to link pi-ru-te and pi-*19-te, two apparently relatively unimportant sites within an area which must have contained a very large number of such sites, ${ }^{219}$ nor does this value give a particularly good explanation for e-pi-*19-ta. ${ }^{220}$ Although $r u_{2}$ can certainly not be ruled out as a possible value for $* 19$, neither at this point can almost any other $C(C) V$ value.

\footnotetext{
${ }^{217} \mathrm{pi}^{*}$-19-te (An 427) is the location of some officials called pu-ka-wo (/purkawoi/ 'fire-kindlers'?), whose reason for being recorded is unclear; at pi-ru-te a group of men called ku-re-we (obscure) are contributing oxen (Cn(1) 3) and soldiers (An 519).

${ }^{218}$ The o-ka tablets, including An 519, refer to troops stationed at the coast, so the majority of places listed are probably in the Hither Province (Bennet 1998:117); two of the other TNs on An 427, a-pu $u_{2}-w e$ and a-kere-wa, are certainly in this province (Bennet 1998:114-20; Cosmopoulos 2006:210-11).

${ }^{219} \mathrm{C} .250$ TNs have been identified in the Pylos tablets, of which almost $50 \%$ are attested only once (Bennet 2011:151, 154).

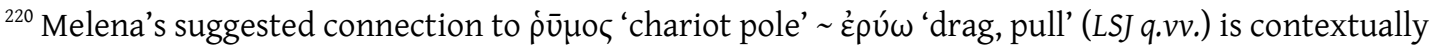
attractive (/epi-rrū-tās/ could be either the pole or the pole-stay) but, although the etymology of żpúw is unknown $(C D E)$, there appears to be little evidence for a geminate - $\rho \rho$ - in this verb in later Greek: such geminates are usually maintained word-medially (Lejeune 1972f:119-20) but compounds of this verb

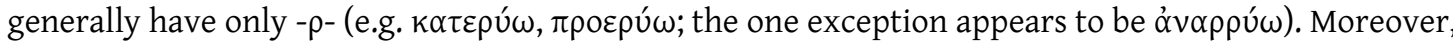
Melena's argument for deriving this from */sr(e)ux-/ to give Mycenaean /-rr-/ involves assuming

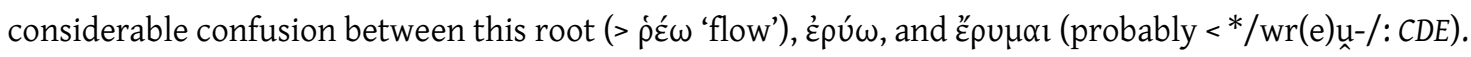
Although such confusion does occur in the case of the last two, both of which have derivatives in both $\dot{\varepsilon} \rho v-$ and $\dot{\rho} \bar{v}-(C D E)$, similar phonetic confusion with $\rho \dot{\varepsilon} \omega$ could not have arisen until after the loss of both */s-/ and $* / w-/$, the latter of which had not yet taken place in Mycenaean.
} 


\section{$\underline{2.3 * 22} \uparrow^{\prime}$}

$*_{22}$ is one of the more frequently attested undeciphered signs, with 70-4 certain or possible examples from Knossos and Thebes and on ISJs, ${ }^{221}$ plus $142-59$ certain or possible examples of the morphologically identical ideogram CAP from Knossos, Thebes, and Pylos. $^{222} * 22 /$ CAP has three main types of palaeographic variation:

1) *22 can have a single vertical stalk, corresponding to the ungendered form of CAP $\uparrow$, or a double stalk, corresponding to $\operatorname{CAP}^{\mathrm{f}} \hat{\Lambda}^{-223}$ the male form $\hat{\neq}$ is not found as a syllabogram. ${ }^{224}$ Both variants of $* 22$ are found at Knossos, where the single-stalk form is slightly more common (42 certain examples in 8-11 identified hands; ${ }^{225}$ the double-stalk has 17 examples in 5-7 identified hands) ${ }^{226}$. H117 is the only scribe attested as using both forms: ${ }^{227}$ this scribe's double-stalk examples are all either majuscule or the first entry on

\footnotetext{
${ }^{221}$ The only other possible example, ${ }^{*} 22$-ne-wa-ta on $\mathrm{KH}$ Ar 4.2, is mentioned only by Olivier 1993:33, who dismisses it; from autopsy this seems extremely improbable, and so is excluded here.

${ }^{222}$ The ideogram *150 [T (only found in the KN Mc-series, H132) has been argued to be formed from a combination of $\mathrm{ra}^{*} 22 / \mathrm{CAP}$ (Bennett 1972:57); however, as Melena 1972:39-40 points out, the differences between H132's forms of $r a$ and *22/CAP and of * 150 show that this scribe, at least, did not conceive of the latter as being a ligature/monogram. This sign will therefore not be included in the discussion here.

${ }^{223}$ Note that the syllabographic examples of this form usually have two strokes which slant or curve down to meet at or near the bottom $(\tilde{\eta})$, whereas CAP ${ }^{\mathrm{f}}$ usually has two stalks slanting away from each other $(\hat{\Lambda})$; the contrast can be seen on KN Mc 1508, which has examples of both. The single example of ${ }^{*} 22$ in a form identical to $\mathrm{CAP}^{\mathrm{f}}(\mathrm{KN} \mathrm{Xd} 131$, from the RCT), plus the LA evidence (p.88) suggests that the double-stalk form of ${ }^{2} 22$ originated in $\mathrm{CAP}^{\mathrm{f}}$ and that this difference may have developed in order to distinguish the two (cf. qi/ovis, n.224 below).

${ }^{224}$ of the other animal syllabograms/ideograms, qi/ovis $\bar{\uparrow}$ appears to be the only one which also has both single- and double-stalk forms used syllabographically (and shows a similar variation between converging and diverging double stalks to *22/CAP); none have examples of the male ideogram form used in this way.

${ }^{225} \mathrm{H103}$, H105, H117, H120, H121, H132, H223, H133?, H217?; RCT: H124-C, H124; 11 unattributed examples (C 5370.B; F(3) 741.2.3.4, 8242.1.2; G 820.3; Wb 5665; X 7553, 7741.a, 9691).

${ }^{226}$ H101, H108, H117, H122, H125, H116?; RCT: H124; 3 unattributed examples (F(2) 841.2; Mc 1508.B [n.227 below]; X 5784).

${ }^{227} \mathrm{H} 132$ uses the single-stalk form; the double-stalk form is found on Mc 1508, attributed (with Mc 5187) to H132? (Olivier 1967). However, Melena 1972:33-4 and Killen 2008:48-9 argue persuasively that this attribution is very unlikely to be correct. 1508 and 5187 were found in the East Wing, while H132's Mc-
} 
the tablet (Dn 1093.1), and there are no examples in this hand of a majuscule single-stalk form, implying that for H117 the double-stalk form is the 'full' or elaborate form to be used in headings, with the simpler single-stalk used in regular entries. Other hands, however, use the double-stalk in minuscule (H101, H122) or the single-stalk in majuscule words/headings (H103, H124-C, H132, H223), so that it appears that in general individual scribes used only one of these two variants; this fits into the frequently-observed pattern of H117's unique usage of both elaborate and simplified forms (p.72). Only the doublestalk form is found at Thebes (where all the examples of *22 are in H305 or H305? apart from Uq 434.1̣, -); all the ISJ examples have a single stalk.

2) Some Cretan examples of both *22 and cAP have a slight curve, C-shape, or oval underneath the head. At Knossos, H107 consistently shows this feature in CAP, and it is also present in a minority of RCT examples (CAP: Ce 163.2, H124-B, 7516.1, H124; *22: Xd 7808, H124) and one of H125's two examples of*22 (V(5) 1002.B; not on V(5) 756.B. As both of these are in majuscule headings, the use of the simpler form is not due to size). There are also three examples by unknown hands (CAP: C 7088, ${ }^{*} 22: \mathrm{F}(2)$ 841.2; X 7655); all certain ISJ examples (all of which originate from Crete) also have this feature. ${ }^{228}$
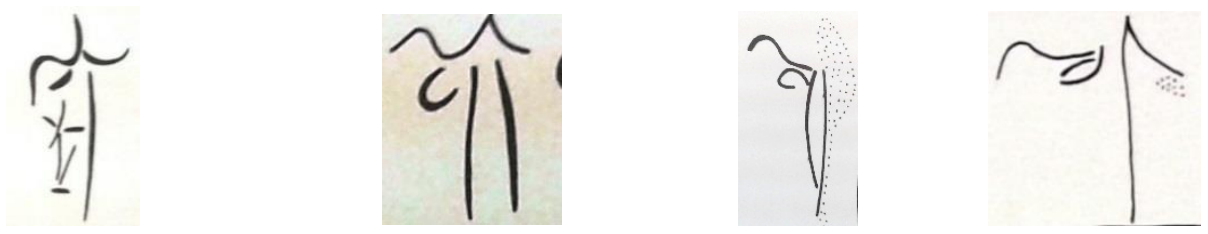

KN C 7088 (-; ki CAP), Co 904.2 (H107), V(5) 1002.B (H125), Xd 7808 (H124) 229

series were found in the Arsenal, and there are significant differences in the forms of other signs, particularly CORN, and in the layout of the tablets (1508 and 5187 are the only two Mc tablets which deviate from an otherwise strict order of listing the four commodities). I therefore suppress the attribution 'H132?'.

${ }^{228}$ The possible example of ${ }^{*} 22$ on a supposed ISJ from Sidon, Lebanon (Doumet-Serhal et al. 2008:32-3) is not yet fully published and so will not be discussed further.

${ }^{229}$ Drawings: COMIK. 
3) *22/cap’s head can be a simple curve, a wavy line, or a curve with a separate vertical stroke at the end:

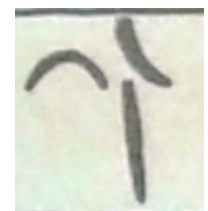

KN Da 1353.b (H117)

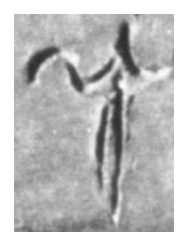

KN Uf(2) 839.b (H122)

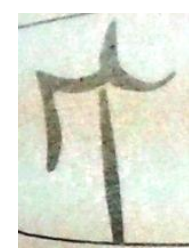

KN Xf $4486(\mathrm{H} 133 ?)^{230}$

At Knossos, these differences do not appear to be diagnostic of scribal hand: in hands with a large number of examples, the shape of the head varies between 'curved' and 'wavy' to a great extent. ${ }^{231}$ The type with the vertical stroke at the end of the head is uncommon at Knossos (*22: Xf 4486, H133?, X 7655, -; CAP: C(4) 912 v.1, H111); as H111 also has examples of the wavy-head type (C(4) 911), this feature likewise appears not to be diagnostic.

Amongst the ISJs, the EL Z 1 form has a wavy head, while those of the ta- ${ }^{*} 22$-de-so group vary between wavy, curved, and curved with an almost-vertical end; all of their heads, however, have an unusual position partway down the stalk.

At Pylos, in contrast to Knossos, the majority of examples of CAP have the vertical stroke at the end of the head, and here this feature does appear to be diagnostic: H1 consistently uses this form over at least 28 examples $^{232}$ (all the examples by unknown

\footnotetext{
${ }^{230}$ Drawings: COMIK; photo: MER.

${ }^{231}$ E.g. H117 writes * 22 with both the curved and the wavy forms of the head; there is no correlation between this and the use of the single- or double-stalk forms, nor with the size of the signs.

${ }^{232}$ The only possible exception is C.AP ${ }^{\mathrm{m}}$ [ on $\mathrm{Cn}$ 285.4, which (from autopsy) does not appear to have a vertical; however, the sign's identification is uncertain due to damage.
} 
hands or attributed only to $\mathrm{Ci}$, Cii, or Ciii also have this form ${ }^{233}$, while H21, H31, and H42 consistently do not (over at least 18, 10, and 2 examples respectively). H21 and H31 show some variation between the more usual wavy head and forms with a (nearly) rightangled bend; since this variation is seen within single hands, and even on a single tablet (Cn 1287, H31), it appears not to be significant.

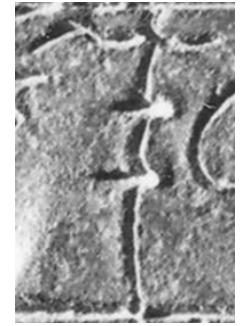

PY Cn 599.1

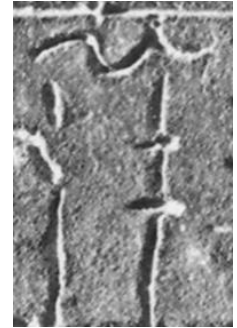

Cn 599.2

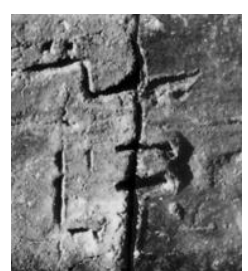

Cc $660 \quad(\text { all H21 })^{234}$

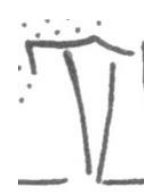

At Thebes, H305's examples of *22 fairly consistently show a (relatively flat) head with a vertical at the end (e.g. Fq 276.7, left), ${ }^{235}$ while both the verticalstroke and the wavy-head form are attested in CAP (the former on Uo 121.b, -,

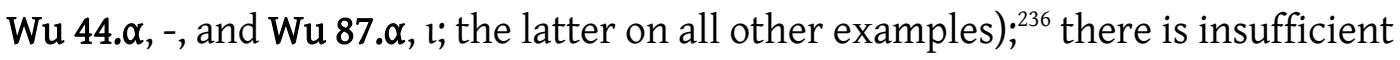
evidence to say whether this is diagnostic as at Pylos, but H305's consistency implies that it may be. Whether this is the case or not, the greater prominence of the vertical-stroke form of the head is clearly a general mainland development.

\footnotetext{
${ }^{233}$ The only possible example by H6 (çAP ${ }^{\times}[$on Un 853.4), if correctly identified, would also have this form.

${ }^{234}$ Photos: MER.

${ }^{235}$ Drawing: $T F C^{3}$. The form on Fq 214.12 is unique in having a 'hook' in place of the vertical. Autopsy showed that the equally unusual form shown in $T F C^{3}$ 's drawing of Fq $245[+] 255.12$ (with a vertical on each side of the head) is inaccurate: this is in fact similar to H305's other examples.

$236 *_{2}^{2}$ on Uq 434.10 (-) is drawn by Aravantinos et al. 2008 with a wavy head; however (although there is considerable damage to the top of this sign) autopsy showed the traces to be at least equally compatible with the $\mathrm{H} 305$ form of 22 (an alternative reading ko also cannot be entirely excluded). The form on Wu 431. $(-)$, which has no head at all apart from a short straight stroke to the right-hand side, is unique and problematic; I tentatively read CAP. (in preference to TFC's CAP ${ }^{\mathrm{m}}$, since it appears that either CAP ${ }^{\mathrm{f}}$ has been corrected to $\mathrm{CAP}^{\mathrm{m}}$ or vice versa) only because any other animal ideogram seems a more difficult reading.
} 
*22/CAP's LA correspondence, AB22, is certainly attested as an ideogram (in the ungendered, female, and male forms) and probably also as a syllabogram Imageremoved (in the female form only): the uncertain attestation of AB22 $2^{\mathrm{f}}$ on HT 101.1 reasons Image (right) ${ }^{237}$ in the sequence AB79-2. $2^{\mathrm{f}}-07$ appears a very probable one. ${ }^{238}$ Although removed for copyright the shape of the head is unusual in LA, it is very close to the LB wavy-head reasons forms; for the indication of the female form by a single short stroke to the right, cf. $\mathrm{AB} 22^{\mathrm{f}}$ on $\mathrm{PH}\left(\right.$ ?) $31 \mathrm{~b} .3$ (left), which clearly contrasts with a preceding $\mathrm{AB} 22^{\mathrm{m}}$. It therefore appears most probable that LB derives its dual usage of *22/CAP from LA. ${ }^{239}$

As said above, AB22 generally has only a simple curved head (drawing 1, below); the wavy-head form and the type with a vertical stroke on the end are both rare but attested (the latter is shown in drawing 2). The 'understroke' seen on a minority of LB examples is, however, a regular feature. There is also a possible simplified form which resembles a shepherd's crook or question mark (drawing 3), which is not paralleled in LB. ${ }^{240}$

\section{I}

\section{mages removed for copyright reasons}
1) $\mathrm{PH}(?) 31 b .2$
2) $\mathrm{PH}(?)$ 31a.4 (AB22 $\left.{ }^{\mathrm{m}}+\mathrm{AB} 81\right)$
3) HT $20.3^{241}$

\footnotetext{
${ }^{237}$ Drawings: GORILA.

${ }^{238}$ GORILA $^{1}$ reads this as -[.]-, but $-22^{\mathrm{f}}$ - is listed in the index of GORILA ${ }^{5}$. Melena 1987b:218-20 also reads AB22 ${ }^{\mathrm{f}}$ (used syllabographically) on HT 135a.3 and AB22 (used syllabographically) on PH 14a and ZA 10a.4, b.6; however, I follow the readings AB21 ${ }^{\mathrm{f}}, \mathrm{A} 304$, and AB81, respectively (GORILA; CTLA).

${ }^{239}$ All the other similar animal ideograms/syllabograms are attested in LA with both functions (AB21 = qi/ovIs, $\mathrm{AB} 23=m u /$ BOS, AB85 $=a u /$ sus $)$.

${ }^{240}$ GORILA marks all examples of this form as uncertain instances of *22, but Younger takes these readings as certain (Younger (LA HT); Younger (LA Other)); it seems a plausible enough type of simplification. ${ }^{241}$ Drawings: GORILA.
} 
It seems that at some stage in the creation or early use of LB, this use of the 'understroke' was largely dropped in favour of a simpler version, and retained by only a few scribes at Knossos (in at least one case, H125, as an optional feature; it is impossible to say whether the use of this feature in the RCT is diagnostic or not) and on ISJs (where the use of elaborate forms may have been encouraged by the difference of medium and in particular the large size of the signs: see Judson 2013:74-5). The absence of this feature on the mainland implies that it was lost altogether by the time of the Pylos and Thebes tablets, if it was ever present on the mainland at all.

\section{Prospects of decipherment}

Despite ${ }^{2} 2$ 's relatively large number of examples, it is not attested in a single vocabulary term, only in PNs and the TN $\mathrm{da}^{*}$ 22-to (and related ethnic adjectives). Some evidence as to its possible value is, however, provided by its distribution, as it very frequently appears adjacent to $-i$ or $-j:^{242}$

Following $-i$ :

]$a-d i-22-s a$

ta-di-*22-so

Preceding $-\mathrm{j}$ :

ko-du-*22-je

*22-ja-ro

]$^{*} 22-j e-m i[$

]-*22-jo

Other:

$d a{ }^{*} 22-$ to (and da-*22-ti-jo/-ja)

${ }^{242}$ See L.R. Palmer 1963b:22-3. This list excludes very dubious and/or isolated examples. 
o- ${ }^{*} 22-d i$

ta- ${ }^{*} 22-\mathrm{de}-\mathrm{so}$

*22-ri-ta-ro

*22-ka-ne

Of the 11 different terms in which ${ }^{*} 22$ is certainly or probably attested, therefore, in c.55\% it either follows $-i$ or precedes $-j$, which might suggest a vocalic value in $-i ;{ }^{243} \mathrm{cf}$. also the two instances in which ${ }^{*} 22-\mathrm{Ci}$ could represent /CCi/, ${ }^{*} 22$-ri-ta-ro and o- ${ }^{*} 22-\mathrm{di}$ $\left(\mathrm{da}^{*}\right.$ 22-ti-jo/-ja is clearly not significant in this respect, since ${ }^{*} 22$ appears preceding -to in the TN). In addition, $k o-d u-{ }^{*} 22-j e-$ likely, from its context as a recipient of HORD, to be a dative - is quite plausibly from an $i$-stem ${ }^{*} k o-d u-{ }^{*} 22 .{ }^{244}$ Of course, neither of these latter points is at all conclusive, and the sample size is small; nonetheless the possibility of a value in $-i$ is worth exploring.

Of the possible values for a sign in -i inherited from LA, $z i, p i_{2}$, and $n w i$ have been shown (pp.64-5) to be the most probable in theory. ${ }^{245} *_{22}$ 's distribution (with at least two wordinitial attestations) might seem to fit zi or $\mathrm{pi}_{2}$ better than $n w i$; of these two, its absence from any apparently Greek vocabulary words might fit zi best, since a sign for / $\mathrm{p}^{\mathrm{h}} \mathrm{i} / \mathrm{could}$ easily be used in representing Greek words. ${ }^{246}$ However, none of these features is remotely conclusive as evidence. No spelling alternations to support any of these values can be found; the best candidate for an alternation involving *22 is the pair of MNs

\footnotetext{
${ }^{243}$ As most attempts at deciphering this sign have assumed, with the exception of the very unlikely $90_{2}$ (Georgiev 1955:7), tra (Georgiev 1956:78), and wre (Gallavotti 1957b:168-9).

${ }^{244} \mathrm{Cf}$. ma-di-je, $i$-stem dative on TH Av 101.5, whose nominative ma-di is found on KN As 603.2 and Db 1168.B (Killen 1999:217; TFC 1 :359); ma-di-jo on KH Z 3 is probably the genitive of the same name (Killen 2015:163). ${ }^{245}$ The suggestions $j i$ and $i_{2}$ (Lee 1962 [unpublished]:1) have been shown to be improbable (pp.10, 24-5). ${ }^{246}$ If ${ }^{*} 22-$-ri-ta-ro and o- ${ }^{*} 22-d i$ are correctly interpreted as /CCi/ clusters, / $\mathrm{p}^{\mathrm{h}} \mathrm{ri}-/$ is the only one of the three possibilities for the first which is phonetically plausible, while none of the possibilities for the second seem particularly likely (T. Meißner, pers. comm.). However, there is evidently no proof that the vowel of $*_{22}$ is in fact a dummy vowel in either of these two terms.
} 
ta- ${ }^{*} 22-d e-s o$ and ta-mi-de-so. ${ }^{247}$ Based on this, the value $m i_{2}$ has often been suggested (e.g. Sittig 1954:68; Landau 1958:13; L.R. Palmer 1963b:22-3; Janda 1986), but this has no specific phonetic value to justify its existence. Melena $1987 \mathrm{~b}$ argues that this alternation actually supports the identification of $*^{*} 2$ as $p i_{2}$, based on his identification of the ' $p_{2}$ series' as originating in a Minoan pre-nasalised phoneme / ${ }^{\mathrm{m}} \mathrm{b} /$ (see also Melena 2014b:71 and forthcoming:8-10): ${ }^{248}$ ta- ${ }^{*} 22-d e-s o$ and ta-mi-de-so, along with a further similar MN tade-so, are cited as fluctuating spellings of non-Greek / ${ }^{\mathrm{m}} \mathrm{b} /$ with $p_{2^{-}}, m-$ and $\varnothing$, but an alternation with $p$ - to give the value $p_{2}$ - is noticeably lacking from this equation. As these MNs certainly cannot refer to the same person, ${ }^{249}$ they seem more likely to be different names, especially as it has already been shown (p.33, n.84) that the only certainlyidentified member of the $p_{2}$-series, $p u_{2}$, offers no evidence supporting this hypothesis (see further p.119ff).

Nonetheless, a vocalic value in -i remains a reasonable, though not certain, possibility for *22. In the absence of secure alternations or vocabulary words containing this sign,

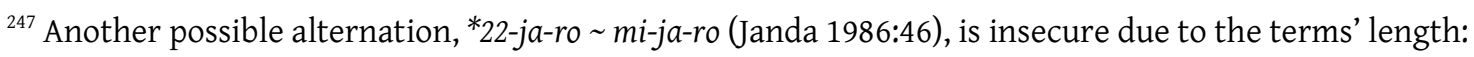
note the existence of at least five other MNs at Knossos in [.]-ja-ro (da-ja-ro, ku-ja-ro, ]ma-ja-ro, pa-ja-ro, te-ja-ro).

${ }^{248}$ The argument of Witczak 1993a that $*_{22}=/ \mathrm{bi} /$ acrophonically from */bisōn/, and that $p_{2}$ - therefore = $/ \mathrm{b}-/$, has already been shown to be completely unsupported (p.28, n.68). The suggestion that ${ }^{*} 22$ was retained specifically in order to represent Minoan / ${ }^{\mathrm{m}} \mathrm{b} /$ (Palaima-Sikkenga 1999:602-3) predates the discovery of the Theban examples of this sign, which (while not ruling out such a status entirely) make it considerably less likely.

${ }^{249}$ ta-mi-de-so is a 'shepherd' at $e-r a(\mathrm{KN} \mathrm{Dl}(1)$ 844.b); ta-de-so probably refers to several different people at Knossos (As(1) 604.2: at ra-su-to; De 1409.B: 'shepherd' at e-ko-so; Df 1285.B: 'shepherd' at ru-ki-to; V(3) 655.3: at ja-po?; $X$ 7558: no context), none of whom can be identified with ta-mi-de-so. ta-*22-de-so appears on the ISJs TH Z 871, 872, [870], 876, and KH Z [5], 39; ta-de-so on TH Z 869 probably refers to the same person (based on the palaeography, particularly the unique form of de [Judson 2013:76], which implies that these inscriptions were all produced by the same painter or workshop, and therefore also refer to the same 'producer'; cf. discussion in Judson 2013:96ff.) but this seems most likely to simply be an error, rather than proof that ta- ${ }^{*} 22-d e-s o$ and ta-de-so are in fact accepted spellings of the same MN.
} 
however, it is not possible either to prove this or to decide between $z i, p i_{2}$, or $n w i$ as potential values.

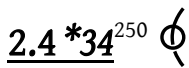

*34 is a relatively frequent sign, with 37-8 certain/possible examples at Knossos, Pylos, and Thebes. ${ }^{251}$ This sign's main palaeographic variation is between the forms previously designated as $*_{34} \phi_{\text {and }} *_{35} \phi_{\text {; }}$ as these two are now officially recognised as the same sign, ${ }^{252}$ they are here referred to as $*^{*} 4 \alpha$ and ${ }^{*} 34 \beta .{ }^{253}$ This variation is found at all three sites and is clearly diagnostic of scribal hand. ${ }^{254} * 34$ also varies in the elements present at the centre of the curve, with forms ranging from a simple semicircle to ones with a small central semicircle or complete circle. All of these forms are found at Knossos, as illustrated below

\footnotetext{
${ }^{250}$ As specified in the Preface, parts of this section are based on an essay submitted to the University of Cambridge in February 2012 as part of the M.Phil. degree; these are included here for the sake of the completeness of this discussion.

251 * 34 is often said to be identical to the ideogram LUNA, 'a month's rations' (Knossos) and therefore to have developed from a depiction of the moon (e.g. Docs²:50; Jasink 2013). LuNA appears in three forms: $($ (the most common), $\mathbb{C}$ and (. Although the last two resemble forms of $* 34$, a simplification of the first (representing a half-moon) into these forms seems more plausible than the opposite development; there are also no examples of LUNA with reversed orientation, nor of AB34 appearing as an ideogram. *34 and LUNA are therefore probably unrelated.

${ }^{252}$ Suggested at an early stage of the decipherment (e.g. Ventris 1953:10) but not universally accepted (e.g. Doria 1972:39-46) until the discovery at Thebes of the MNs o-*34-ta-o (gen., of 33.1) and o-*35-ta (nom., Ug 3): the former is listed as the head of a wool workshop alongside another individual called di-u-ja-wo (of 33.2), who recurs in the Ug-series (di-wi-ja-wo, Ug 11) in an identical capacity to o-*35-ta (both are involved in transactions of the obscure commodity 0 ), suggesting that o-*34-ta-o and o-*35-ta are in fact the same person (TT II:96-7; Duhoux 1983:114-5). The decision that both signs should be transcribed as *34 was taken at the $11^{\text {th }}$ Mycenological Colloquium (Austin, Texas, 2000) and reaffirmed at the $13^{\text {th }}$ Colloquium (Olivier 2012a:581). On the chronological implications of this identification, see pp.242-3.

${ }^{253}$ The sign with a circle in the centre of a straight vertical on PY La 626.a (H13) has been tentatively identified as *34 (PT II; PTT) but is far more likely to be rọ ${ }_{2}$ (alternative suggestion by PTT, adopted in PoNIV. This sign's usual form is $\$$, but a form similar to that on La 626.a is found in KN H206).

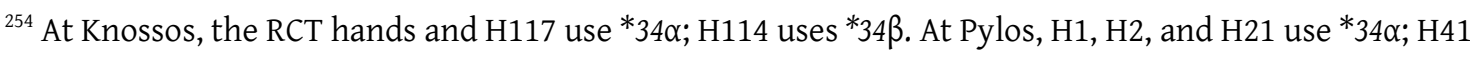

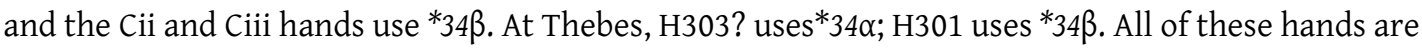

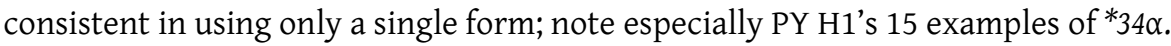


(note the characteristic variation between minuscule and majuscule in H117, and the presence of only the simplest two forms - plain curve or with central semicircle - in the $\mathrm{RCT}$ ); only the form with the central circle is found on the mainland. There is no correlation between these forms and the sign's orientation.

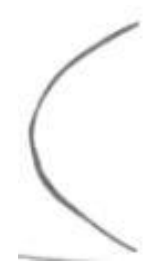

H124-G (KN Sc 235) H117 (Dv 1239.1)

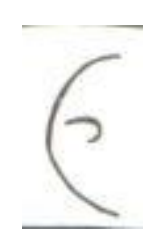

H117 (Da 1253.B)

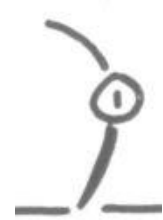

H114 (Ld 787) $)^{255}$

*34's LA antecedent, AB34, has only seven certain attestations, all of the *34 $\alpha$ orientation; forms include a simple curve or one with a central dot, horizontal stroke, or vertical, similar to the first three LB forms above (the central circle of the H114/mainland form may be a continuation of the LA form with a central dot or a development from the form with a semicircle).

\section{Images removed for copyright reasons}

\section{KN Zc 6.1 HT 116a.4 HT 140.1 ZA 6a.1-2 $2^{256}$}

There is only one possible example of $A B 34 \beta$, on the gold ring $\mathrm{KN} \mathrm{Zf} 13 \quad$ Image (right) ${ }^{257}$ this reading is suggested by CTLA (L69) as a possible alternative to AB8̣1 군 (L98), read by both CTLA and GORILA. The latter reading does seem removed for copyright reasons preferable, since the two short strokes on the horizontal are not paralleled in

\footnotetext{
${ }^{255}$ Drawings: COMIK.

${ }^{256}$ Drawings: GORILA.

${ }^{257}$ Drawing: GORILA.
} 
AB34, whereas there are examples of AB81 appearing as a simple curve with a horizontal, sometimes with bars on the ends (below); although characteristically AB81's horizontal crosses the semicircle, this difference is perhaps explicable by the sign's small size and the medium.

\section{Images removed for copyright \\ reasons}

\section{HT 127b.4 AP Za 2.1 $1^{258}$}

There is thus no positive evidence for the existence of AB34 $\beta$. Nonetheless, this type of variation is reasonably frequent in LA, ${ }^{259}$ very probably due to LA's flexibility in writing direction (sinistroverse, ${ }^{260}$ boustrophedon, ${ }^{261}$ and 'divergent ${ }^{262}$ LA inscriptions are all attested in non-administrative contexts); ${ }^{263}$ by contrast, there is no good evidence for LB being written in any direction other than dextroverse, ${ }^{264}$ and sign-reversals are much less common than in LA. ${ }^{265}$ An LA origin therefore still seems the most probable explanation for *34's mirror-image variants.

\footnotetext{
${ }^{258}$ Drawings: GORILA.

${ }^{259}$ E.g. AB60 $\Delta, A B 67$ 壬, and AB76 \(are amongst the LA signs which most frequently appear reversed.

${ }^{260}$ VRY Za 1; PL Zf 1; PO Zg 1 (Dimopoulou et al. 1993).

${ }^{261} \mathrm{KN} \mathrm{Za} \mathrm{19;} \mathrm{IO} \mathrm{Za} 11$.

${ }^{262}$ IO Za 9 (Karetsou et al. 1985).

${ }^{263}$ Janko 1987:316.

${ }^{264}$ The only possible example of sinistroverse LB is TH Z 868, reading ru-i but very probably intended to have the same inscription as TH Z 866 and 867 (i-ru); however, the combination of several unusual palaeographic features on these inscriptions suggest this is unlikely to be genuine sinistroverse writing (Judson 2013:78). The Kafkania pebble has been interpreted as boustrophedon (Arapogianni et al. 1999:40); however, following Palaima 2002-2003, I do not regard this as genuine LB.

${ }^{265}$ Reversals similar to ${ }^{*} 34 \alpha / \beta$ are seen in the metrograms $v$ and $Q ;$ tu and the ideogram CYP/PYc both have variants in which the stroke(s) at the top curve either to the right or the left, but it is debatable whether these are cases of sign-reversal or merely the different arrangement of a single element (cf. Driessen 2000:123 on tu). Compare also the formation of $d w o \vec{\Delta} \vec{\Delta}$ and $d w e$ ifs from mirror-image forms of wo $\vec{\Delta}$ and we 2 (pp.34-7), as well as the occasional reversals of other signs: e.g. pu, MY Go 610.4 and PY An 39.1 (Palaima
} 


\section{$\underline{\text { Prospects of decipherment }}^{266}$}

Although *34 does not have a particularly large number of examples, it is attested in a relatively high number of vocabulary words:

\section{Nouns:}

*34-ki-no-o, nom.pl., construction material ${ }^{267}$

*34-ka-te-re, agent noun in /-tēr/, probably nom.pl. objects (Killen 2000:145-8); ${ }^{268}$ probably not related to ${ }^{*} 34-k e$-te-si (pace DMic) ${ }^{269}$

*34-ke-te-si, dat.pl., title or group designation in /-tēr/; recipients of a do-so-mo, probably a group of cult personnel ${ }^{270}$

*34-to-pi, instr.pl., obscure, but associated with $a-k e-a_{2} /$ aggeha/ 'jars'271

\section{Adjectives:}

${ }^{*} 34-k a\left[\right.$ and $a-*^{*} 34-k a$, both describing textiles - the latter is plausibly the negative of the former

1981:80); KN H133's form of do (Olivier 1967: Table XXXIV); and perhaps mịi, MY Ge 603.1 (TITHEMY). On reversed signs on ISJs, see Judson 2013:77-8.

${ }^{266}$ Decipherment attempts which treat ${ }^{*} 34$ and ${ }^{*} 35$ separately can now be dismissed (e.g. Furumark 1954 , Part I:112: ${ }^{*} 55=$ ar?, ${ }^{*} 34$-; Lurja 1955: ${ }^{*} 34=p a_{4},{ }^{*} 35=e_{2}$; L.R. Palmer 1955b:44: $*_{34}=a i_{2},{ }^{*} 35=p u_{3}$ ), as can various suggestions which have little contextual, linguistic, or theoretical support: e.g. me (Sittig 1954:68; Georgiev 1956:63-7); $r$ /l (Gallavotti 1956b); pra (Gallavotti 1961; Perpillou 1984); $t u_{2}=/ \mathrm{t}^{\mathrm{h}} \mathrm{u} /$ (Doria 1972:39-40). The two most widely-quoted proposals, $r u_{2}=/ \mathrm{lu} /$ and $a_{5}=/$ hai/, will be discussed below.

${ }^{267}$ For buildings (Baumbach 1972) or ships (Hocker-Palaima 1990-1991).

${ }^{268}$ For other interpretations (nom./instr.; dual/pl.; referring to people or objects) see, e.g., Docs²:348; Carlier 1984:100-1.

$269 * 34-r a-k a-t e-r a$, also on Va 15 , is generally interpreted as being the acc.sg./pl. of the same noun (e.g. Killen 2000:145), but from autopsy I regard the *34- as very doubtful. Even if this were confirmed, for these two to be the same word would require either an omission from *34-ka-te-re or plene spelling of syllablefinal /-r/ or /-1/ in *34-ra-ka-te-ra.

${ }^{270}$ Docs $^{2}: 279$; L.R. Palmer 1963b:221-4.

${ }^{271}$ For various interpretations of the contents and purpose of this tablet see, e.g., L.R. Palmer 1963b:369-70; Galaty 2007:76-7; Killen 2007:117; Palaima 2014. 
*34-ke-u, m.nom.sg., describing a tripod

$\mathrm{pu}_{2}{ }^{*} 34-[$.$] ? - perhaps describing textiles (p.80, n.207).$

None of these, however, can be certainly identified from context, and so any decipherment proposal based purely on etymological interpretations of these is doubtful (the possibility of spelling alternations will be discussed below). The most widely-quoted such decipherment, $r u_{2}=/$ lu/ (Blegen-Lang 1960:162-3; L.R. Palmer 1963b:23-4; Ruijgh 1979; Duhoux 1983), is largely based on the assumption that since this adjective appears in the same context as $a_{3}-k e-u-$ Pylos H2's Ta-series, describing a tripod - and the latter is generally interpreted as /aigeus/ 'decorated with a goat', *34-ke-u ought to be a contrasting adjective referring to a different form of animal decoration. This is therefore interpreted as either /lukeus/, 'decorated with a wolf' or /lugkeus/ 'decorated with a lynx'. The insecurity of this is adequately demonstrated by the two different possible interpretations given for ${ }^{*} r u_{2}-k e-u$, parallel to the wide variety of interpretations given to other terms in the corpus (*34-to-pi, for instance, is interpreted by both Ruijgh 1979:567-8 and Duhoux 1983:117 as /lutorp ${ }^{\mathrm{h}} \mathrm{i}$, , but explained as 'people who have been released from an obligation' or as a term describing vases 'with sieves', respectively), not to mention that signs representing specifically /l/ (or $/ r /$ ) have been shown (p.65) to be improbable.

The distribution of *34 might provide some help, as it is word-initial in $60 \%$ of the terms in which it is found (68\% of occurrences), and of the six terms in which it appears medially, two are plausibly prefixed $\left(a-{ }^{*} 34-k a\right)$ or a compound (au-to-*34-ta-ra). This distribution makes a palatalised value or one in $n w$ - unlikely, though not impossible (cf. pp.74-5); although *34's 
word-initial percentage is not as strikingly high as that of $a_{3}$ or au (pp.17, 22), ${ }^{272}$ it might still suggest a vocalic value, in which case the most likely possibilities would be ei or oi (pp.25-6). Neither diphthong, however, produces any particularly satisfactory Greek readings; ${ }^{273}$ and both run into problems with $a^{-}{ }^{*} 34-k a$, which would have to be assumed not to be an example of the negative prefix - not impossible, but difficult since ${ }^{*} 34-k a[$ is attested in a very similar context. ${ }^{274}$

The second widely-accepted decipherment proposal starts from this basis and therefore suggests an aspirated value for ${ }^{*} 34, a_{5}=/$ hai $/{ }^{275}$ This would solve the difficulty of $a$-*34-ka, and is also based on some possible alternations with $a_{3}$ : $a_{3}-k e-u \sim *^{*} 34-k e-u$ (i.e. assuming these two terms found in identical contexts to be equivalent rather than contrastive, as in the /lu/ theory above; note though that they are both written by H2); ${ }^{276} a_{3}-k i-n o-o ~ \sim ~ * 34-k i-n o-o$ (part of a chariot, Knossos construction material, Pylos: contextually possible but not certain). ${ }^{277}$ Although these alternations appear quite attractive, various other factors make the value /hai/ an extremely improbable one. ${ }^{*} 34$ has a secure LA correspondence, and is

\footnotetext{
${ }^{272} \mathrm{Cf}$. also the LA distribution: only two of AB34's five certain attestations are word-initial, but this is a very small corpus.

${ }^{273}$ There are few possible alphabetic comparanda for any of these terms if $e i$ or oi is substituted for * 34 , and those that do exist are unconvincing in context: for instance, *34-ke-u could be compared to oikeúc and *34to-pi to öïocóc, but the description of a tripod as 'belonging to the household' is not particularly informative, and the association of *34-to-pi with jars makes 'arrows' an unlikely meaning.

${ }^{274}$ Both are on Ld(2)-series tablets by H114. $a-{ }^{*} 34-k a$ describes TELA+PA; although the ideogram following *34-ka[ is lost, it is preceded by *161, which also appears on both of the tablets containing $a{ }^{*}{ }^{*} 34-k a$ TELA+PA. ${ }^{275}$ See in particular Melena 2013; also, e.g., Docs'2:23, 349, 504; Meriggi 1955:73; Melena 1987a:615, n.9, 2014b:16, 89, and forthcoming:114-8 (also quoted in Duhoux 1983:12-45); Palaima 2014:88; Bibee-Wilson-Wright 2015. ${ }^{276}$ As discussed in more detail below (pp.117-18), there are a few examples of scribes at Pylos alternating between core and extra signs, even in the same term; in fact $\mathrm{H} 2$ shows such an alternation in the Ta-series (we- $a_{2}-r e-j o$, Ta $714.1 \sim w e-a-r e-j a, 642.1$ ), so this alternation is certainly not ruled out. However, the fact that the majority of the scribes who use $*_{34}$ also use $a_{3}$, even in terms which (according to Melena's interpretations) would originate from the same root (Melena forthcoming:18-9), may be more problematic.

${ }^{277}$ I exclude $a_{3}$-to $\sim *_{34}$-to (MN, Knossos; obscure term, Pylos) and $a_{3}$-te $\sim *_{34-t e}$ (name of official; appellative, both Pylos) due to their short length.
} 
found reasonably frequently at Knossos, both features which contrast with the only known aspirated sign, $a_{2}$; in fact, ${ }^{*} 34$ 's distribution also differs significantly from $a_{2}$ 's, since the latter is not particularly frequent word-initially (p.15). A third doublet of $a$ (or a doublet of the doublet $a_{3}$ ) is implausible on economic and structural grounds (/hai/ could already be represented by $a(-i), a_{2}(-i)$, or $a_{3}$, while a sign for this value would represent two different phonemic features, aspiration and an -i diphthong). Finally, the Greek interpretations which are supposed to justify this value involve reconstructing aspiration in roots which never contain it in later Greek (e.g. /aig-/ 'goat'); the argument that these words contain secondary, demarcative aspirations, perhaps deriving from a non-phonemic glottal stop, ${ }^{278}$ is an utterly circular one with no good linguistic basis, nor an explanation as to why a sign representing a non-phonemic feature should exist. The value /hai/ should therefore be rejected.

An alternative proposal which would still take into account *34's distribution would be the value zu. As previously argued (pp.11-12), such a sign's primary use in Greek would be in the ø-grade of /z(e)ug-/, and it might therefore have a high proportion of wordinitial occurrences; however, given the possibility of its occurrence medially in compounds of this root and non-Greek names, this proportion might well be lower than that of a vocalic sign - which corresponds exactly to *34's distribution (p.96). In this context it is also noticeable that, in a reasonably high proportion of the terms in which *34 appears, it is followed by -k-, as it would have to be if representing /zug-/: ${ }^{*} 34-k a[$,

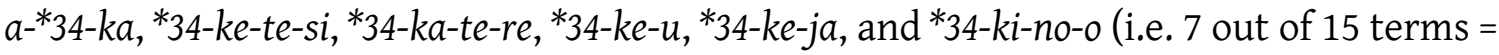
47\%). In practice, however, the potential Greek results this value produces are not much

\footnotetext{
${ }^{278}$ Melena 2013:222-3. The argument of Bibee-Wilson-Wright 2015 that this glottal stop is the PIE laryngeal $* h_{2}$ is based entirely on comparisons with Egyptian and Ugaritic and lacks any foundation in actual LB or IE evidence.
} 
better than those for ei or oi: for instance, $a^{*}{ }^{*} 34-k a$ could be /azuga/, *34-ka[ a corresponding positive adjective such as */zuga[ios]/, ${ }^{279}$ and ${ }^{*} 34-k e-u * /$ zugeus/, but these are largely without alphabetic parallels (’’̧uyos is attested, but only at a fairly late period [LSJ q.v.]; $\alpha^{\prime} \zeta u \xi$ is attested in classical Greek, ${ }^{280}$ but similarly has no corresponding positive form), rely in one case on a restoration, and are not easily interpretable in context (it is not at all clear what '(un)joined/paired' textiles or a 'joined' tripod would actually be). There is therefore no better evidence to assign the value $z u$ than there is for ei or oi, although none of these values can be excluded. It is also possible, however, that the sign's distribution may be due either to chance, or to a non-phonetic factor (in a sample of this relatively small size, the existence of several terms deriving from the same root could skew both the distribution and features such as the relatively high frequency of the sequence ${ }^{*} 34-k$ - - as was argued relating to $z u$ ). If this is the case, then of the various values shown to be the most probable inherited ones (pp.64-5), *34 might most plausibly be a member of the $p_{2}$-series (as argued on p.96, the sign's distribution makes palatalised values and $n w$ - less likely, while its appearance in apparently Greek terms makes zi equally unlikely); but again, none of the possible $p_{2}$ - values produce any plausible results. Ultimately ${ }^{*} 34$ 's possible values simply cannot be narrowed down any further.

\footnotetext{
${ }^{279}$ I owe the suggestion of this particular form to R.J.E. Thompson (pers. comm.).

${ }^{280}$ Suggested by T. Meißner (pers. comm.), along with an alternative suggestion that /azuga/ could instead be formed with the copulative $\alpha-(<* /$ smo-zug-s/, 'joined together'; cf. the Hesychian gloss ö

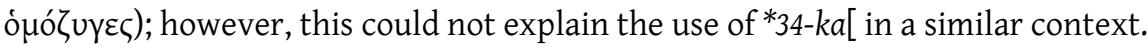




\section{$2.5 * 47$ 必}

*47 has 15-23 certain/possible attestations, all of which are at Knossos apart from one very probable example at Mycenae. ${ }^{281}$ Its basic form is a diagonal cross with a circular element in the centre; the simpler forms have 2-4 small curved strokes forming a ring around the centre, while the more complex form has a circle surrounded by small curved or straight strokes. ${ }^{*} 47$ also varies in the presence or absence of small strokes on the top ends of the cross. ${ }^{282}$ These variants are distributed as follows:

\section{Simpler form:}

KN H138: 4 small curves (e.g. Fp(1) 1.9, right); ${ }^{283}$ no end-strokes

KN H115: 3 small curves (top and sides); no end-strokes

MY H57: 3 small curves (top and sides); end-strokes (see pp.101-2)

KN H224: 2 small curves (sides); end-strokes

Elaborate form:

KN RCT: circle + 2-3 small curves (sides or top and sides); end-strokes

KN H141: circle + 3 small curves (top and sides: Fh 393, 5479?) or small strokes (Fh 351, $5430,462.2 ?){ }^{284}$ end-strokes

\footnotetext{
${ }^{281}$ There is also now at least one very probable example from Ayios Vasileios (Vasilogamvrou 2015); as this is not yet fully published it is excluded from the discussion here.

${ }^{282} \mathrm{Cf}$. Melena forthcoming:21-2.

${ }^{283}$ Photo: author (NAM).

${ }^{284}$ This scribe's single example of ${ }^{*} 47$ with no central circle, only the surrounding small strokes (Fh 357.1), appears to be a one-off due to lack of space.
} 


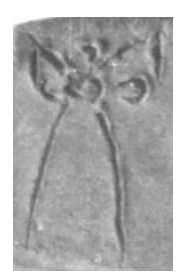

Fh 393

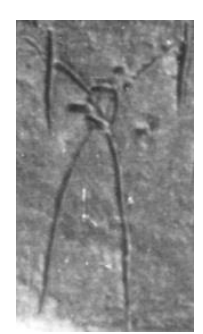

Fh $351^{285}$

This sign's structure and variation is very similar to that of $j e$ ' $X$, which consists of a plain diagonal cross with end-strokes, optionally with a central circle or two small curves at the sides.je's more complex form is therefore identical to some H138 or H224 forms of *47, and the two can only be distinguished in some instances by comparing their forms within individual scribal hands ${ }^{286}$ For instance, H115 and H138's consistent lack of endstrokes on 47 seems to be a means of distinguishing this from je (which always has these strokes), although both of these scribes also use the simpler form of je; H141, who writes the more complex form of $j e$ and always includes the end-strokes on *47, distinguishes the two through the addition of extra small curves or strokes at the centre of *47.

The third sign in the MN me-ta-[.]-wa (MY Go 610.1), identified as *47 by MT II, Imageremoved for MT IV, and SCM, was re-identified as je in TITHEMY. The copyright reasons drawing in SCM (right) shows a form compatible with either reading, with end-strokes and two small curves in the centre; however, autopsy of the tablet showed what appears to be a third small curve at

\footnotetext{
${ }^{285}$ Photos: MER.

${ }^{286}$ Nonetheless, the two are still distinct signs: individual hands do generally have a consistent distinction between them, and their distributions also differ significantly, with only one known term, je-zo, in which je appears word-initially, while 47 is almost invariably initial (Chadwick-Killen 1965:95-6). There is therefore no support for the suggestion that these are variants of a single sign (Grumach 1963:157; cf. Meriggi 1955:74; Driessen 2000:120).
} 
the top of the sign (left), ${ }^{287}$ making this virtually certainly an example of *47 (cf. KN H115).

It has been argued that the ideogram *141/AUR 'gold' ' $\$$ is either identical to *47 or a monogram of this plus another sign (Lee 1962 [unpublished]:4; Witczak 1992b). However, although the examples of this ideogram without the top element (PY Tn 316, 996) resemble the simpler form of $* 47$ without end-strokes, it is more probable that these are a simplification of $\mathbb{W}$ than that the latter is an elaborated form of $* 47 ;^{288}$ a monogram is equally unlikely as this top element does not resemble any LB syllabogram.

All the LA examples of AB47 $\not$ are of the simpler type, with only a circle in the centre, ${ }^{289}$ while AB46 Xalways takes the form of a cross with 'feet', with no central element. ${ }^{290}$ Thus, either of these LA forms could formally correspond to LB je; AB47 is identified as such because it also corresponds to the simpler type of LB *47, while there is no LA sign corresponding to the more complex type of this sign. Rather than the elaborate form of *47 representing an original form which underwent simplification, it therefore seems as though the more elaborate forms may have developed within LB: hands which adopted a more elaborate form of ${ }^{*} 47$ would then have also been able to add a central circle to je without risking confusion between the two signs. ${ }^{291}$ As the elaborate * 47 is already

\footnotetext{
${ }^{287}$ Photo: author (NAM).

${ }^{288}$ Godart 2009:112-4 suggests that this simpler form is a separate ideogram, representing silver, but although a decision was taken at the 2010 Colloquium to designate this as *141bis (Olivier 2012a:582), this decision was reversed by the CIPEM signary committee at the 2015 Colloquium.

${ }^{289} 8$ examples in GORILA, plus one on MIL Zb 1 (Niemeier 1996).

${ }^{290}$ 8-9 examples in GORILA, plus ARKH Zc 8 (Sakellarakis-Sapouna-Sakellaraki 1997:332, fig.295); PE Zg 6.2 (E. Hallager 2012: 267-9, fig.3); THE Zb 6 (Boulotis 2008:69-70, fig.8 $\alpha-\beta$ ).

${ }^{291}$ The opposite process - influence from * 47 causing a circle to be added to je, thus necessitating greater elaboration in the former to distinguish the two - is also possible (Driessen 2000:120) but would require a period of even greater overlap between the two forms.
} 
attested in the RCT, this development must have taken place very early in LB, or even perhaps very late within LA.

\section{Prospects of decipherment}

As *47 is attested only in PNs and TNs, many if not all of which are likely to be non-Greek in origin, its chances of decipherment are low; ${ }^{292}$ no particularly secure spelling alternations are attested. ${ }^{293}$ Its largely word-initial distribution - of 23 certain or possible examples, $16(70 \%)$ are certainly or probably initial, and no more than three (13\%: $a^{*} 47$, $a^{-}{ }^{*}+7-w i\left[{ }^{294}\right.$ and $\left.m e-t a-{ }^{*}+7-w a\right)$ are certainly medial - might suggest a vocalic value, ${ }^{295}$ in which case either ei or oi would be the most probable value; ${ }^{296}$ the lack of examples of *47 followed by - $j$ - is not problematic given the similar situation of $a_{3}$ and $r a_{3}$, which show an apparent preference for the use of $-a$ - (with or without $-i-$ ) before $-j-{ }^{297}$ As in the case of

\footnotetext{
${ }^{292}$ Most decipherment proposals are based purely on insecure comparisons of one or more terms in * 47 's corpus with other PNs or TNs and/or the assumption that $* 4=$ AUR, and produce values of improbable types or with no specific phonetic value: e.g. bre (Gallavotti 1958c:54); $w a_{2}=$ /wa/ (Georgiev 1956:79; cf. Sittig 1954:69); $k r u$ (Landau 1958:13); $k u_{2}$ (Lee 1962 [unpublished]:4); dwi (Owens 1991-1993:265); $a_{4}$ (Witczak 1992b).

${ }^{293} \mathrm{me}^{-t a-}$ - $47-w a$ (MN, Mycenae) could be compared to me-ta-ka-wa (MN, Pylos: Doria 1972:37-8) or ]me-ta-ra-wo[ (MN, Knossos: Doria 1972:38-9) but evidently neither of these is remotely secure, nor are $\mathrm{ka}_{2}$ or $\mathrm{ra}_{4}$ plausible values. Janda 1988 compares *47-so-de to ]da-so-de, both TNs in the KN Fh-series, but this relies on a comparison of two terms of two syllables (-de, as the allative particle, being excluded), one of which is an uncertain reading; in any case it is unclear what sound-value a sign $d a_{2}$ could have.

${ }^{294}$ These two terms may in fact be the same word: both are possible MNs in the KN Sc-series (RCT).

${ }^{295}$ Although theoretically this distribution could also point to a value $z u$ (p.12), this sign has neither a significant number of instances followed by $-k-$, nor any attestations in probable Greek vocabulary words, so there is no strong reason to assign it this value (though it is not excluded).

${ }^{296}$ The suggestion that ${ }^{*} 77$ is $j i>h i / z i$ (Melena forthcoming:22; cf. Doria 1972:36-7, $i_{2}$ ) is based on Melena's proposal of the same value for *63, only found on the mainland, and the assumption that * 47 could be *63's Cretan equivalent. This is undermined by the example of the former at Mycenae (as well as at Ayios Vasileios), and is moreover implausible for many of the same reasons as Melena's suggestion of /hai/ for *34:ji has been shown to be unlikely to exist (p.10), while the sign's distribution, LA antecedent, and frequency of occurrence at Knossos argue against an aspirated value. In any case Chapter 1 also showed that there are good reasons for assuming the potentially-useful value $h i$ - and, similarly, other aspirated vowel doublets - not to exist (pp.24-25). See further pp.127-8.

${ }^{297}$ See pp.17-18; p.20, n.47.
} 
*34, however, this proportion of word-initial occurrences is not quite high enough to make a vocalic value certain; AB47 also shows a rather different distribution, with only two word-initial occurrences out of seven attestations in sign-sequences, though this is an even smaller corpus than that of ${ }^{4}$. Like *34, if this sign is in fact a $C(C) V$ sign, it is less likely to be a palatalised sign or one in $n w^{-}$, so a value in either $z$ - or $p_{2}$ - might be the most probable. Ultimately, however, the lack of any attestations in vocabulary words means that no progress can yet be made on deciphering this sign - though it is to be hoped that this may change with the publication of the tablets from Ayios Vasileios. ${ }^{298}$

\section{$2.6 * 49 \pi$}

*49 is a very rare sign found only at Knossos, ${ }^{299}$ with 4-6 examples. ${ }^{300}$ Its basic form consists of three straight or slightly curved verticals surmounted by a wavy horizontal line; H117 and H119 have two small strokes at the bottom of each vertical, while H103 has a simpler version without these strokes.

\footnotetext{
${ }^{298}$ The discovery of this sign at Ayios Vasileios, together with the demonstration that there is very probably an example at Mycenae, make it increasingly likely that its lack of certain non-Knossian examples up to this point has been due merely to chances of attestation.

${ }^{299}$ The only suggested example at another site, *49-ne-wa-ta (KH Ar 4.2) is mentioned only by Olivier 1993:33, who dismisses it; from autopsy I judge this to be highly improbable and therefore exclude it here. $300 * 49$ has occasionally been suggested to be a variant of pu (Georgiev 1956:79-80) or *63 (Meriggi 1955:75). However, there is a clear palaeographic distinction between ${ }^{*} 49$ and $p u$ in the two hands in which both are attested, H103 and H117: the top of $p u$ is formed by a stroke which curves down to form the sign's lefthand leg, while the top of ${ }^{*} 49$ is clearly separated from the rest of the sign (cf. Melena forthcoming:23). Similarly, ${ }^{*} 49$ and ${ }^{*} 63$ are superficially similar (a wavy top with three verticals and some cross-strokes) but differ considerably in their details and formation, so it is better to view *63 as a mainland development unrelated to the (apparently) Cretan *49 (see p.126).
} 

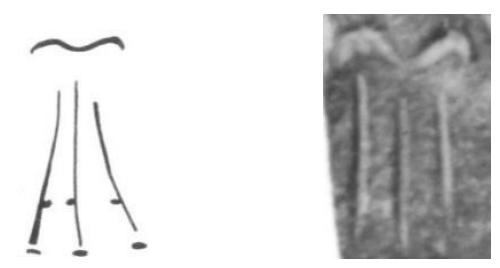

\section{KN Da 1588.B (H117) V(4) 653.2 (H103)}

As both of H103's examples of *49 are minuscule, but all of H117's and H119's are majuscule, it cannot be determined whether this variation is diagnostic of scribal hand or determined by the size of the sign; it seems plausible that H117 might also have used the simpler form in minuscule, since this type of variation is characteristic of this hand (cf. ${ }^{*} 18,{ }^{*} 22$, and $*^{*} 4$ ). Of these three scribes attested as writing $* 49, \mathrm{H} 103$ and H117 are the two most prolific hands at Knossos, while H119 is closely related to H117 in subjectmatter (the former's single example of $\left.{ }^{*} 49,\right]^{*} 49$-so on $\mathrm{Dk}(2)$ 1068.B, is probably the same MN as H117's ]*49-so on Dv 1328). ${ }^{302}$ Despite its rarity, therefore, it appears to have been a sign in mainstream use (unlike, e.g., twe, found only in So-series tablets: p.38) but clearly one that was rare enough for the chances of finding it in a few months' worth of tablets by any less prolific scribe to be very low.

*49's LA antecedent, AB49, is equally rare, with only 4 attestations; since all of these have at least one stroke on the end of each vertical, the form used by H103 probably originated as a simplification of an original form similar to that seen on HT 120.4 (the ZA 10b.2 and PE 2.4 form, with shorter verticals on either side, is not paralleled in LB).

\footnotetext{
${ }^{301}$ Drawing: COMIK; photo: MER.

${ }^{302}$ In both cases the name refers to a 'shepherd' located at ku-ta-to with 114 sheep; Killen 1964:9-11 demonstrates that H119's Dk(2)-series are shearing records relating to the same flocks as H117's Da-Dg tablets (cf. Melena forthcoming:24).
} 


\section{Images removed for copyright reasons}

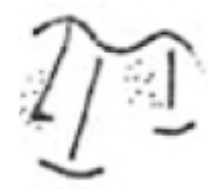

HT $120.4 \quad$ HT 126a.4 ZA 10b.2 PE 2.4

\section{Prospects of decipherment}

As *49 is attested no more than 6 times, probably in 5 different PNs, none of which are long enough to make any possible spelling alternation secure, its chances of decipherment are extremely small. Its distribution (2 word-initial examples, 4 certainly or probably medial) might suggest a $C(C) V$ value rather than a vocalic one; as previously discussed in several cases, the existence of the initial examples argues against a palatalised one and also makes $n w$ - perhaps less likely, leaving a value in $z$ - or $p_{2}$ - as the most probable ones for a sign inherited from LA, ${ }^{304}$ but without spelling alternations or examples in Greek vocabulary words, this cannot be confirmed, nor can anything be said about the sign's possible vocalism. ${ }^{305}$ If any of the undeciphered signs were retained to represent a Minoan phoneme, ${ }^{*} 49$ would appear to be the best candidate for this status, since it is certainly inherited from LA and has no certain or possible examples outside of

\footnotetext{
${ }^{303}$ Drawings: GORILA, except PE 2 (Tsipopoulou-Hallager 1996:32, fig. 12a).

${ }^{304} \mathrm{Both}_{\mathrm{pe}}$ and $\mathrm{po}_{2}$ have been tentatively suggested, the former by Melena forthcoming:25-6 (based on a completely insecure comparison of $t u-{ }^{*} 49-m i$ to the group of PNs beginning tu-ma-, tu-da-, tu- ${ }^{*} 56-:$ see p.118ff), the latter by Owens 1991-1993:265 (on the grounds that the $p_{2}$-series would represent a 'Minoan' phoneme, with which * 49 's distribution is compatible; since $p u_{2}$ is clearly used in a wide variety of Greek words, this is hardly a basis for assigning a $p_{2}$-value).

${ }^{305}$ Melena forthcoming:26 argues that ${ }^{*} 49$ is most likely to have a vocalism in $-a$, as this is the vocalism of the majority of its possible comparanda at Knossos (e.g. *49-sa-ro could be compared to a-sa-ro, ja-sa-ro, and $k a-s a-r o$, and ta-*49-ro to ta-ta-ro and ta-za-ro). Comparisons with other vowels are, however, equally possible (e.g. no-sa-ro, ta-pe-ro) and in any case a decipherment cannot be based on a small number of PNs containing hypothetical Minoan onomastic elements; nor is either of the values suggested, but rejected, by Melena under this hypothesis (pta and wya) particularly likely to have been inherited from LA (pp.64-5).
} 
Knossos; ${ }^{306}$ however, since there are no more than 6 known examples of * 49 , this distribution is at least as likely to be due to this very rare sign's chances of attestation.

\section{$\underline{2.7 * *_{56}}|=|$}

*56 is the most frequently attested of the undeciphered signs, with 75-100

certain/possible attestations; it is also the most widely attested, found on Crete at Knossos and Khania (and on Cretan ISJs), and on the mainland at Pylos and Thebes. ${ }^{307}$ The form of this sign is a 'ladder' with two long verticals joined by three short horizontals positioned in the middle $(|\equiv| \equiv) ; *_{56}$ is therefore distinguished from the similar sign ja horizontals, with two each at the top and bottom). ${ }^{308}$ The only form of palaeographic variation shown by ${ }^{*} 6$ is occasional differences in the number of horizontal strokes: there are examples with two horizontals (KN H117, $\left.{ }^{309} \mathrm{TH} \mathrm{H} 309\right)^{310}$ and a single example with four (KN H111) $)^{311}$. This is H309's only example of *56, but both H117 and H111 also use the regular form of this sign (H117 at least 25x, H111 once), ${ }^{312}$ so this variation does not appear to be palaeographically significant.

\footnotetext{
${ }^{306}$ Cf. Owens 1991-1993:265; Melena forthcoming:25.

${ }^{307}$ There is one possible example from Mycenae, ]-ki-ni-*56 (MY Oe 111.4, H51: TITHEMY), but following autopsy I regard the reading -ja (SCM) as much more likely (although it has only three horizontals, their positioning is more like that of the horizontals in ja; cf. Melena 1987b:204 for other instances of ja by H51 with fewer than four horizontals).

${ }^{308}$ There are occasional examples of $j a$ with three horizontals, but their positioning towards the ends of the sign normally makes it clear that these are not *56: cf. n.307 above, as well as the term ku-ja-ni (also, but less plausibly, read ku-* $\left.{ }^{*} 6-n i\right)$ on the ISJs TH Z 844 and 848.

${ }^{309} \mathrm{KN}$ Dd 1218.B; Df 1222.B, 1230.B; Dg 1226.B.

${ }^{310}$ TH Gp 157.1.

${ }^{311} \mathrm{KN}$ C(4) 911.12.

${ }^{312}$ H117: D-series, passim. H111: C(4) 912.10.
} 
*56's LA antecedent, AB56, is attested up to 46 times and is essentially identical in form; ${ }^{313}$ there is one known example with four horizontal strokes (AP Za 2.1) but none with only two. ${ }^{314}$ H111's form might therefore be inherited, but seems just as likely to be the result of a sporadic independent elaboration; the form with two horizontals is easily explained

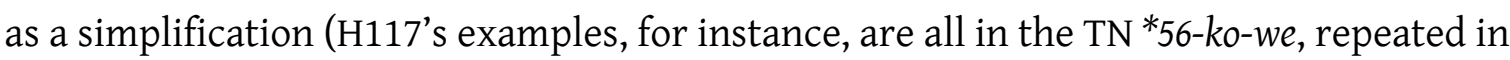
minuscule form at least twenty times in this hand's D-series).

\section{Prospects of decipherment}

Despite its relatively frequent attestations, ${ }^{*} 56$ is found in just two vocabulary words, each with only a single example: $k u-r u-s u-{ }^{*} 56$ (nom.sg. noun: a type of vessel $=* 207^{\mathrm{VAS}}, \mathrm{KN}$

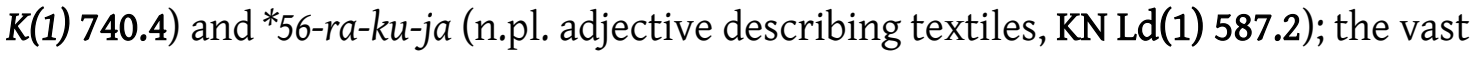
majority of its attestations are in PNs (including possible theonyms) and the TN *56-ko-we (and related ethnic adjectives). The sign's distribution (word-initial in 39\% of the identifiable terms in which it is attested, medial in 43\%, final in 11\%; $7 \%$ uncertain) suggests a $C(C) V$ value rather than a vocalic one; as in several previous cases, this distribution combined with its inherited status suggests a probable value in $z$ - or $p_{2^{-}}$, palatalised values and $n w$ - being rendered less likely by the significant proportion of initial attestations.

\footnotetext{
${ }^{313}$ Up to 43 examples in GORILA, plus SY Za 4 and 5 (Muhly-Olivier 2008:207-9) and MIL Zb 1 (Niemeier 1996).

${ }^{314}$ The form of AB55 (= LB nu) is two verticals with two straight horizontals, as opposed to the semicircles of LB nu: presumably therefore AB56 would always be written with at least three horizontals to distinguish it from AB55.
} 


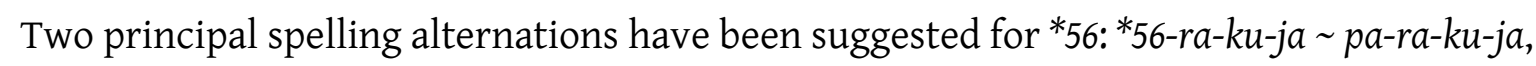
leading to a proposed identification as $\mathrm{pa}_{3} ;{ }^{315} *_{56-r u-w e} \sim$ ko-ru-we, leading to a proposed identification as $\mathrm{ko}_{2} \cdot{ }^{316}$ Each of these proposals will be discussed in turn, starting with the latter as this is the more recent.

\section{${ }_{56}=\mathrm{ko}_{2} ?$}

This proposal is based on the identification of ${ }^{*} 56-r u-w e$ (PN or theonym, dat.) and ko-ru(-we) (PN or theonym, nom./dat.) ${ }^{317}$ as referring to the same individual at Thebes. ${ }^{*} 56-r u-w e$ is found mainly as a recipient of VIN in the Gp-series (H306, H308, H309), ${ }^{318}$ with only one example not from this series (Fq 205.4, H307); ${ }^{319}$ ko-ru(-we) appears mainly in H305's Fq-series (receiving HORD), ${ }^{320}$ with a single other example on Av 101.5 (H304). There are therefore no examples of the same scribe writing both ko-ru(-we) and *56-ru-we, and H305 and H306 consistently use the same form at least six and five times respectively. $T F C^{1}: 207-8,359$ therefore argues that these are different spellings of the same name in complementary distribution by scribal hand, and moreover that as both

\footnotetext{
${ }^{315}$ L.R. Palmer 1955b; Docs ${ }^{2}$; Lejeune 1972b; Consani 1981; Melena 1987b; Kyriakidis 2007. This value would be parallel to $p u_{2}$, but the transliteration $p a_{3}$ is used as $p a_{2}$ was the original transliteration of ${ }^{*} 16=q a$. ${ }^{316}$ Aravantinos et al. 1995:829-33; Lejeune-Godart 1997; TFC 1 :359-60. For arguments against this proposal see, for instance, Melena forthcoming:27-31; Palaima 2006; Kyriakidis 2007.

${ }^{317}$ For varying interpretations of the status of the recipients in the Fq- and Gp-series, see TFC'; Palaima 2006; Killen 2006.

${ }^{318}$ I exclude Gp 114 (H306) from this discussion as it reads only ]ru-we.

${ }^{319}$ I class this tablet as Fq as I do not regard this series attribution as secure. Neither this nor H307's only other attributed tablet, $\mathrm{Fq}$ 207, has any ideograms preserved apart from the metrograms $\mathrm{V}$ and $\mathrm{z}$, which could refer to either dry or liquid measures. These series attribution is therefore presumably made on the basis of the recipients on both tablets: the only series they all have in common is the Fq-series, but some are also found in the $\mathrm{Ft}$ - and $\mathrm{Gp}$-series (and possibly the Gf-series).
}

${ }^{320} \mathrm{Fq}$ 117.2, 126.3, 214.3, 254.4, 284.2, 331.2. I exclude Fq 169.[3]], 241.[3]], and 309.[3]] (also H305) from discussion as these read only ]ru-we. 
are found alongside some of the same other recipients (ke-re-na-i, mo-ne-we, to-pa-po-ro-i, and $a-k o-d a-m o / a-k o-r o-d a-m o)^{321}$ they refer to the same individual.

A closer examination of the contexts in which these recipients are attested, however, offers little support for this identification. ${ }^{322}$ ke-re-na-i, mo-ne-we, and to-pa-po-ro-i each appear once on the same tablet as ko-ru(-we) and ${ }^{*} 56-r u-w e,{ }^{323}$ but although in some instances these are relatively closely associated by the ordering of the tablets (ke-re-na-i appears immediately after both ko-ru [Fq 126.3] and *56-ru-we [Gp 176.a]; mo-ne-we immediately precedes *56-ru[-we on Gp 110.2), in others they are much less so (mo-ne-we and ko-ru are ten lines apart on Fq 254.4 and .14; to-pa-po-ro-i and *5̣-ru-we may well have been separated by one other recipient, not preserved, on Gp 184.1.2; on Av 101.5 and .6b, to-pa-po-ro-i and ko-ru-we are separated by the totalling formula and the relationship between their entries is unclear). If e-pi-do-ro-mo is included, this is separated from ko-ru by seven lines on Fq 254.4 and .11, and perhaps from *56-ru-we by three lines on Fq 205.1 (e-pi-dọ[-ro-mo) and .4. Finally, the link via $a-k o-d a-m o / a-k o-r o-d a-m o$ is based entirely on the assumption that these similarly represent two different forms of the same PN, referring to the same individual: ${ }^{324} a-k o-d a-m o$ is attested only alongside $k o-r u(-w e)$ (Av 101, Fq 117, Fq 254; in each case between one and five recipients are listed between the two), ${ }^{325}$ and a-ko-ro-da-mo only alongside *56-ru-we (immediately preceding it on Gp 164.2). The identification of these two as the same individual is, like that of ko-ru(-we) and *56-ru-we, based on the fact that a-ko-da-mo is found in tablets by H304, H305, and H311

\footnotetext{
${ }^{321}$ Another possibility, e-pi-do-ro-mo, could also be added to this list, though this relies on a restoration see below.

${ }^{322}$ Cf. Kyriakidis 2007:218-22.

${ }^{323} \mathrm{TFC}^{1}: 208$.

${ }^{324}$ Aravantinos et al. 1995:838; TFC $^{1}: 169-70$.

${ }^{325} \mathrm{Fq} 241$ is excluded as this involves a restoration of ko-]ru-we; cf. p.109, n.320.
} 
$\left(\mathrm{Av}^{-}, \mathrm{Fq}-\right.$, and Ft-series $){ }^{326}$ while $a-k o-r o-d a-m o$ is found twice in the $\mathrm{Gp}$-series (Gp 164.2, H306; Gp 215.[2], 327 -): thus these two are said also to be in complementary distribution by scribal hand. TFC'1:170-1 interprets $a-k o-(r o-)-d a-m o$ as /Agoro-dāmos/, 'assembler of the people', but this interpretation is difficult to explain within the usual rules of Greek compound formation, ${ }^{328}$ and in any case no good explanation can be found for /Agoro-/ being spelt a-ko- in the majority of its occurrences. As García Ramón 2006:50 points out, it would still be possible for these two terms to represent the same name if one were a metathesised form of the other, e.g. a-ko-ro-da-mo /Akrodāmos/ a-ko-da-mo /Akordāmos/; such liquid metathesis is not unknown in Mycenaean, but (like most processes of metathesis) is highly irregular and sporadic. ${ }^{329}$ Since contextual evidence to link these two PNs is entirely lacking, ${ }^{330}$ it is much more likely that $a-k o-d a-m o$ and $a-k o-r o-d a-m o$ are in fact different names. ${ }^{331}$

There are therefore no more than four recipients forming a possible association between *56-ru-we and ko-ru(-we); considering the entire list of recipients - of which there are 51 -

\footnotetext{
${ }^{326}$ H304: Av 101.4. H305: Fq 117.[1], 240.1, 241.1, 253.1, 254.2, 257.1, 258.[1], 275.[1], 307.[1], 309.1, 343.[1].

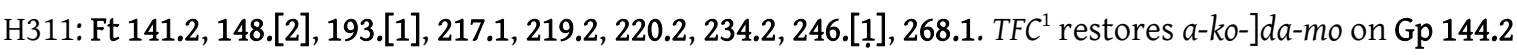
(H305?), but a restoration $a-k o-r o-] d a-m o$ seems more logical given that this name is already attested in the Gp-series (Palaima 2006:140).

${ }^{327}$ The restoration $a$-]ko-ro-da-mo seems plausible.

${ }^{328}$ The proposed interpretation requires the initial element to be verbal rather than nominal, but an ograde verbal element would normally form the second element of the compound, e.g. */Dāmāgoros/; if appearing initially it would normally be in the e-grade, e.g. */Ageredāmos/ (García Ramón 2006:45-50; cf. Palaima 2006:140).

${ }^{329}$ Thompson 2002-2003:355-62.

${ }^{330}$ Only one other recipient, $a-m e-r o$, is associated with both $a-k o-d a-m o$ (Gp 215.1 and .2) and $a$-ko-ro-da-mo (on Fq 254.2 and .5), and the latter in particular is not at all a close association (the two are three lines apart on a tablet listing at least 27 recipients).

${ }^{331}$ E.g. /Ark ${ }^{\mathrm{h}}$ odāmos/ and /Akrodāmos/, as suggested by García Ramón 2006:48-50.
} 
attested on the same tablet(s) as either of these two names shows the overall evidence for their identification as the same person to be extremely weak: ${ }^{332}$

\begin{tabular}{|c|c|}
\hline Recipients found with ko-ru(-we) & Recipients found with ${ }^{*} 56-r u-w e$ \\
\hline \multicolumn{2}{|l|}{$a-k e-n e-u-s i$ x2 +1 } \\
\hline & $a-k o-r o-d a-m o$ \\
\hline \multicolumn{2}{|l|}{$a-k o-d a-m o \times 2+[1]$} \\
\hline \multicolumn{2}{|l|}{$a-m e-r o \times 2$} \\
\hline \multicolumn{2}{|l|}{$a-n u-t o$} \\
\hline \multicolumn{2}{|l|}{$a-p u-w a$} \\
\hline \multicolumn{2}{|l|}{$a-r a-o \times 1+1$} \\
\hline \multicolumn{2}{|l|}{ ]ḍa-ro } \\
\hline \multicolumn{2}{|l|}{ de-u-ke-nu-we } \\
\hline \multicolumn{2}{|l|}{ de-u-ke-we ${ }^{333}$} \\
\hline \multicolumn{2}{|l|}{ do-ra- $a_{2}-j a$} \\
\hline \multicolumn{2}{|l|}{ do-ro-jo } \\
\hline \multicolumn{2}{|l|}{$e-p e-t o-i x 2$} \\
\hline e-pi-do-ro-mo & e-pi-dọ[-ro-mo \\
\hline \multicolumn{2}{|l|}{$e-p i-q \underline{0}[-i$} \\
\hline \multicolumn{2}{|l|}{$i-q o-p o-q o-i \times 2$} \\
\hline \multicolumn{2}{|l|}{]$j a-s o-r o[$} \\
\hline \multicolumn{2}{|l|}{ ka-ne-jo } \\
\hline & $k a-s a[$ \\
\hline & ka-șị [ \\
\hline$k a-w i-j o$ & \\
\hline
\end{tabular}

${ }^{332}$ Table includes all recipients (with at least two signs preserved) found on the same tablet as a certain or probable attestation of either ko-ru(-we) or *56-ru-we (excluding those which read only ]-ru-we: Fq 169, 241, 309; Gp 114).

${ }^{333} \mathrm{TFC}^{1}: 390$ suggests that this and de-u-ke-nu-we are alternative spellings of the same MN, presumably representing different syllabifications /-ke|nwei/ vs. /-ken|wei/ (vel sim.); see also Duhoux 2008:389 (who also has an alternative suggested reading de-u-ke-șe-we). However, the only plausible noun type in this case would be an $\bar{e}$-stem (suggested to me by T. Meißner), whose existence in Mycenaean is uncertain (though a-re /Arei/ is often identified as such: e.g. Heubeck 1971a: 14-17; Risch 1987:291; Bartoněk 2003:263). It seems at least as probable that these are two different names (since de-u-ke-we is the more common, it is unlikely to be an error for de-u-ke-<nu->we). 


\begin{tabular}{|c|c|}
\hline ke-re-na-i & ke-re-na-i[ \\
\hline \multicolumn{2}{|c|}{ ko-du-*22-je x1 + } \\
\hline \multicolumn{2}{|l|}{$k o-w a \times 1+1$} \\
\hline & ku-no \\
\hline \multicolumn{2}{|l|}{ ku-ro 2} \\
\hline \multicolumn{2}{|l|}{$m a-d i-j e \mathrm{x} 1+2$} \\
\hline & $m a-k a \times 3$ \\
\hline \multicolumn{2}{|l|}{ me-to-re-i } \\
\hline \multicolumn{2}{|c|}{$m i-r a-t i-j o \mathrm{x} 1+1$} \\
\hline \multirow[t]{2}{*}{ mo-ne-we } & mo-ne-we \\
\hline & na-ne-mo \\
\hline \multicolumn{2}{|c|}{$o-k o-w e-i \mathrm{x} 1+[1]$} \\
\hline \multicolumn{2}{|l|}{$o-p o-r e-i \times 3+[1]$} \\
\hline \multicolumn{2}{|l|}{ o-to-ro-no } \\
\hline \multicolumn{2}{|c|}{$o-u-w a-j a-w o-n i \mathrm{x} 1+1$} \\
\hline \multicolumn{2}{|l|}{ pi-ra-ko-ro x2 } \\
\hline & ]pọ-me-ne \\
\hline \multicolumn{2}{|l|}{$p u_{2}-k e-q i-r i-n e$} \\
\hline & qe-re-ma-o x1 + [1] \\
\hline \multicolumn{2}{|c|}{ ra-ke-(da-)mi-ni-jo x1 +[1] } \\
\hline & $s a-[]-.j o$ \\
\hline \multicolumn{2}{|l|}{ te-ka-ta-si } \\
\hline & te-ri- $a_{2}[$ \\
\hline \multirow[t]{2}{*}{ to-pa-po-ro-ị[ } & to-pa-po-ro-i \\
\hline & to-jo $\times 1+2$ \\
\hline \multicolumn{2}{|l|}{ to-tu-no } \\
\hline & wa-do-ta \\
\hline \multicolumn{2}{|l|}{ wo-ko } \\
\hline & $z o-w a$ \\
\hline *63-te-ra-de x3 & \\
\hline
\end{tabular}


Sharing no more than 4 out of 51 associated recipients, each of which appears alongside ko-ru(-we) and *56-ru-we only once, is hardly enough to prove identity; in the context of two tablet series which are characterised by the frequent recurrence of recipients and by a very high rate of variability in the composition and ordering of the groups found on

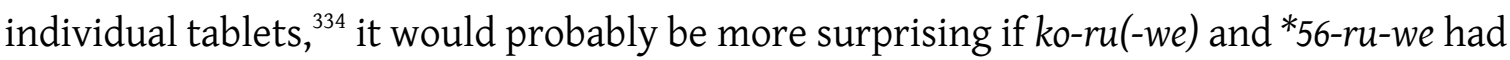
no associated recipients in common at all. Note that the two scribes (H305 and H306) who write these terms repeatedly have each certainly contributed to only one series (Fq and Gp, respectively): ${ }^{335}$ the attested distribution of the terms *56-ru-we and ko-ru(-we) is thus far more likely to result from the existence of two individuals, one of whom regularly receives barley, the other of whom regularly receives wine, ${ }^{336}$ with two different scribes dealing with these two different sets of allocations, than it is from the existence of a single individual with two alternate spellings of their name. ${ }^{337}$ No other good spelling alternations between *56 and ko can be found; of the possibilities listed by Aravantinos et al. 1995:829-33, , $^{338}$ the only ones involving complete or possibly complete terms of more than two syllabograms are ${ }^{*} 56-$-ri-to $\sim$ ko-ri-to and $]{ }^{*} 56-$ so-jo $\sim$ ko-so-jo. The former are incompatible, since ${ }^{*}$ 66-ri-to is an MN at Knossos (Db 1423.B; Xe 6020?) while ko-ri-to is a PN at Pylos (Ad 921; ko-ri[ on Nn 831.1 may well be a PN, but is incomplete); the latter are both MNs listed as owning female slaves at Knossos, but ] ${ }^{5}$ 66-so-jo has an

\footnotetext{
${ }^{334} \mathrm{Cf}$. Kyriakidis 2007:222.

${ }^{335} \mathrm{Gf}$ 134.2, Gp 129, and Gp 144 are attributed to H305?, and Fq 200 to H306?. However, I follow James 20022003:399, n.9 in regarding 200 as probably not part of the Fq-series, since neither its formatting nor its entries match other tablets in this series. With only four signs (and one erased sign) on Gf 134.2, its attribution to H305? (while the rest of the tablet is, like the other Gf-series tablets, unattributed) must be extremely tentative; the same goes for the attributions of Gp 129 (8 signs) and 144 ( 6 signs). Note also that although both of these are palm-leaf tablets of similar appearance to the rest of Gp-series, neither has the vin ideogram attested.

${ }^{336}$ Although if the series attribution of $F q 205$ is correct, ${ }^{*} 56$-ru-we would be receiving barley in this case (see p.109, n.319).

${ }^{337}$ /-us/ is a common PN ending in Mycenaean, and there are several other disyllabic names in -ru in the corpus, so it is not implausible that there should be two different disyllabic names in -ru at Thebes (Kyriakidis 2007:222-3).

${ }^{338}$ See also Lejeune-Godart 1997; $T F C^{1}: 359$.
} 
unknown number of slaves located at Amnisos (Ai 1036, H204) while ko-so-jo has a woman and her two daughters, possibly at ka-ma (Ap 637, H103), so there is nothing to show these to be the same person. In any case, since both MNs are in the genitive, these are in fact two-syllable terms, and therefore too short for a secure alternation (as LejeuneGodart 1997:267-8 admit) - a problem that applies equally to *56-ru-we and ko-ru(-we). Finally, no precise phonetic value has actually been suggested for $*_{56}=k o_{2}$, and neither of the obvious possibilities $\left(/ \mathrm{k}^{\mathrm{h}} \mathrm{o} /\right.$ or $\left./ \mathrm{koi} /\right)$ are at all plausible as sound-values, so this decipherment proposal cannot be supported on any grounds.

\section{${ }^{*} 56=p a_{3} ?$}

The value $p a_{3}=/ p^{\mathrm{h}} \mathrm{a} /$ is, however, a very plausible one in principle. The alternation pa-ra-ku-ja $\sim{ }^{*} 56-r a-k u-j a$ is also contextually good, since both are neuter plural adjectives describing textiles (TELA) in the KN Ld(1)-series:

KN Ld(1) 575.a ẹpẹe-si-ja

.b pa-we-a , / pa-ra-ku-ja TELA2 $30 * 1581$

KN Ld(1) 587.1 to-sa, po-ki-ro-nu-ka TELA 24 re-u-ko-nu-ka TELA ${ }^{2} 372$

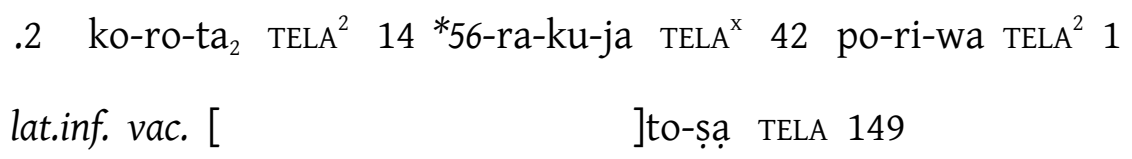

Moreover, both probably refer specifically to the colour or decoration of the textiles. $p a-r a-k u-j a$ appears to be related to the noun *pa-ra-ku (attested in the instrumental singular pa-ra-ku-we/pa-ra-ke-we), a material used for decorating furniture in the PY Taseries, parallel to ku-wa-no /kuwanos/ = kúavos - 'lapis lazuli' and/or 'blue glass’339 and

\footnotetext{
${ }^{339}$ See Bennet 2008.
} 


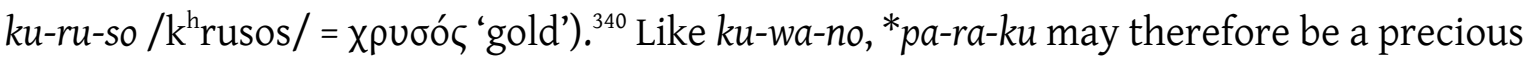
stone or coloured glass; ${ }^{341}$ in either case, the most plausible textile-related meaning of pa-ra-ku-ja would be 'of pa-ra-ku colour' or perhaps 'decorated with pa-ra-ku [beads/platelets]'. ${ }^{342}$ pa-ra-ku-ja also appears in the same position as other colour/ decoration terms (e.g. compare pa-we-a e-qe-si-ja re-u-ko-nu-ka, $\operatorname{Ld}(1) 571$ and 572), while at least three of the other adjectives on $\operatorname{Ld}(1) 587$ similarly refer to colour or decoration. ${ }^{343}$ Even stronger contextual evidence is given by the relationship between Ld(1) 575 and 587: the similarity in the number of cloths recorded, the different descriptions of cloth given, and the ratio of these different types of cloth suggest that 587 is the 'totalling' tablet for a set of delivery records (represented by Ld(1) 598) which record the same cloth as the $\operatorname{Ld}(1)$ 'store' records, of which 575 is one. ${ }^{344}$ As Kyriakidis 2007:225-6 points out, the only types of cloth found on $\operatorname{Ld}(1) 587$ but not in the 'store' records are po-ri-wa (clearly a rare type since there is only one listed on 587) and *56-ra$k u-j a$, whose 42 cloths are roughly comparable to the 30 pa-ra-ku-ja cloths on 575: this gives further support for seeing the ${ }^{*} 56-r a-k u-j a$ and the $p a-r a-k u-j a$ cloths as the same items, and the two terms as therefore being variant spellings of the same word.

\footnotetext{
${ }^{340} p a-r a-k u$ appears as a probable MN on PY Cn 201.1, and without context on KN Xf 5102; it is not clear whether these are related to $\mathrm{pa}-\mathrm{ra}$-ku-we etc.

${ }^{341}$ For the latter suggestion, see Hughes-Brock 2011:102; Piquero 2015:119-20. See further p.119.

${ }^{342}$ Melena 1987b:212; Barber 1991:313, n.2; Hughes-Brock 2011:102 (though the suggestion that *pa-ra-ku could be related to $\pi \lambda \alpha k$ - and mean 'platelet' cannot apply unless ${ }^{*} 56-r a-k u-j a$ is not the same word, since this would give no reason for the use of $p a_{3}$ ). The suggestion that $p a-r a-k u(-j a)$ refers to cloth decorated with very fine wool (Kyriakidis 2007:227) is based on a very uncertain reading of pa-ra-ku on KN Od 667.B, which in any case could also be interpreted as a colour term (Firth-Nosch 2002-2003:137). pa-ra-[.]-we-jo, possibly pa-ra-ku-we-jo (KN Sp 4451), may also be related to *pa-ra-ku but is probably an adjective of material.

${ }^{343}$ Melena 1987b:211-12.po-ki-ro-nu-ka = /poikil-ŏnuk ha/ (either 'multi-coloured' or 'decorated with multicoloured o-nu-ka'); re-u-ko-nu-ka =/leuk-ōnuk ${ }^{\mathrm{h}} \mathrm{a}$ ( (either 'white/undyed' or 'decorated with white o-nu-ka'); po-ri-wa = /poliwos/, 'grey'. On ko-ro-ta 2 , see pp.52-3.

${ }^{344}$ Killen-Olivier 1968:119; Killen 1979:151-2.
} 
The only potential difficulty with this alternation is that $\operatorname{Ld(1)} 575$ and 587 are attributed to the same scribe, H116, and it might seem prima facie unlikely that a single scribe should spell the same word in two different ways. ${ }^{345}$ There is no other instance in which H116 has alternated between a core sign and an extra one in this way, ${ }^{346}$ nor are there many examples of other scribes alternating between using $p u$ and $p u_{2}$ to represent $/ p^{h} u$ / even in different terms; ${ }^{347}$ there are, however, occasional examples of scribes alternating between other extra signs and their core equivalents even in the same word, ${ }^{348}$ and other, more striking forms of variation in single words is not unknown. ${ }^{349}$ That H116

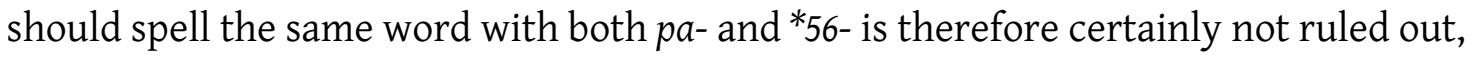
but nor does this type of variation appear to be particularly common. A full survey of orthographic variation, clarifying the extent to which individual scribes use varying spellings, would enable a more informed judgement as to the probability of this alternation, but this is beyond the scope of this thesis. Similarly, without such a survey it is difficult to know how far it should be taken as significant that of the 19 scribes who have certainly written $* 56,5(26 \%)$ have also certainly used $p a$ for $/ \mathrm{p}^{\mathrm{h}} \mathrm{a} /$ in terms such as pa-i-to / $\mathrm{P}^{\mathrm{h}}$ aistos/, pa-we-a /p ${ }^{\mathrm{h}}$ arweha/, pa-ki-ja-na /Sp $\mathrm{p}^{\mathrm{h}}$ agiana/, etc. (KN H103, H116, H117,

\footnotetext{
${ }^{345}$ Olivier 1967:58-60 in fact regards the tablets ascribed to 'H116' as possibly by two or even three different scribes due to some differences in formatting and sign-forms; however, the single attribution of $\mathrm{H} 116$ is retained as there is no certain way of distinguishing these possible hands. In any case, $\operatorname{Ld}(1) 575$ and 587 both fall into the same group even under Olivier's tentative division of the H116 tablets. As Olivier 1967:59 points out, there is some difference in the way that the $r a$ and the $k u$ are written in *56-ra-ku-ja and $p a-r a-k u-j a$, but this is not sufficiently conclusive to attribute these tablets to different hands.

${ }^{346}$ Although variation in the spelling of ke-se-ne-wi-ja/ke-se-nu-wi-ja is attested in this hand, and is one of the reasons for the suggestion that 'H116' in fact includes tablets by more than one hand - see n.345 above. ${ }^{347}$ Two scribes at Knossos who are attested as using $p u_{2}$ have (probable) examples of $p u=/ p^{h} u /$ (H101: pu-te, probably / $\mathrm{P}^{\mathrm{h}} \mathrm{utēe} /$, although possibly /Put ${ }^{\mathrm{h}} \mathrm{e} s / ;$ H136, pu-ta-ri-ja / $/ \mathrm{p}^{\mathrm{h}}$ utaria/); two scribes at Pylos attested as using $p u_{2}$ have less secure possible examples (H2: pu-te-u, / $\mathrm{P}^{\mathrm{h}}$ uteus/ or /Puteus/?; H15, pu-ti-ja perhaps $=p u_{2}-t i-j a / \mathrm{P}^{\mathrm{h}} \mathrm{ut}^{\mathrm{h}} \mathrm{iās} /$ ?). Cf. Duhoux 1986:148-9.

${ }^{348}$ E.g. PY H1, o-ka-ra $a_{3} \sim o-k a-r a$ (An 657.4 and .13 - i.e. not just the same term but also on the same tablet); H2, we- $a_{2}-r e-j o \sim ~ w e-a-r e-j a / w e h a l e y o s,-a /(T a ~ 714.1,642.1) ; \mathrm{H} 26$, we-je-ke- $a_{2} \sim$ we-]je-ke-a (Sa 787.A, 791, 843; Wa 1148 . The restoration is very plausible since Wa 1148 , like the Sa-series, probably relates to wheels). ${ }^{349}$ E.g. the alternation between ko-ri-ja-da-na and ko-ri- $a_{2}$-da-na by H57 on MY Ge 605.3B, .4B and .5, which actually marks a difference of pronunciation.
} 
H136; PY H41); this is quite a lot higher than the proportion of scribes who have certainly written $a_{2}$ who have also used $a$ for $/$ ha/ $(3 / 29=10 \%$ : PY H1, H2, H26), or that of those writing $\mathrm{pu}_{2}$ who have also certainly used $p u$ for $/ p^{h} u /(2 / 20=10 \%$ : see p.117, n.347) but it is entirely possible that factors such as preferred or standard spellings for common terms could cause different extra signs to have very different usage rates. Of the various other possible alternations of $*_{56} \sim p a$ which have been proposed in support of this identification, ${ }^{350}$ the only reasonably good possibility is ka-ra-*56-so $\sim k a-r a-p a-s o$, given the length of the terms and the fact that both are MNs at Pylos. Although there is no direct proof that the two refer to the same individual (the former is a landholder on Eo 269 1.s., H41, and En 659.19, H1 ${ }^{351}$ the latter is a bronze-smith on Jn 389.2, H2) it is possible that they do given the apparently significant overlap between individuals appearing in the Jn- and E-series (Nakassis 2013:100-2); without such an identification they could still be examples of the same name, though even this is far from being certain. pa-pu-so $\sim *^{*}$ 6-pu-so $\sim *^{*} 56-p o-$ so is rather less good, since these, respectively, are an MN on PY Jn 415.1 and perhaps KN Xf 4495 (pa-pu[); an MN on TH Wu 45. $\beta$; and a WN on KN Ln 1568.1b.

In addition to these possible $*_{56} \sim$ pa alternations, Melena 1987b:209-18 suggests several possible alternations between ${ }^{*} 66$ and $m a$ (whose interpretation will be discussed below). The best of these is tu-ma-da-ro tu- ${ }^{*} 56-d a-r o$ : these are both 'shepherds' at Knossos, located respectively at da-wo (KN Db 1368.B, H117) and e-rạ (Dv 1370.b, H117); Melena also adds a possible shortened form tu-da-ra (another 'shepherd', location unknown, on

\footnotetext{
${ }^{350}$ See L.R. Palmer 1954:67; Melena 1987:212-18. The other proposed alternations all involve uncertain attestations of $*_{56}$ and/or pa, or incomplete or very short terms.

${ }^{351} \mathrm{NB}$ that based on autopsy of En 659.19 I would read only ka-ra-[ (vest. in broken area compatible with -*56-sọ), but the contextual similarities still guarantee that this refers to the same person as Eo 269 1.s. (and note that the traces are not compatible with -pa-sọ).
} 
Do 924.B, H106). The first two of these are at least relatively long, but are clearly not the same individual, and note that both are written by H117. ${ }^{352} a-*_{56-n o} \sim a-m a-n o$ are both MNs on KN As(2) 1520.13 and v. (H105), the former also being found on KN Dv 5232 (H117); although occasional examples of the same term being spelt in different ways on a single tablet are attested (p.117, n.348), it seems more likely that these are different names, particularly as they are only three syllabograms long. None of the possible ${ }^{*} 56 \sim$ ma alternations are nearly as contextually secure as *56-ra-ku-ja pa-ra-ku-ja.

Any proposal to decipher ${ }^{*} 6$ as $p a_{3}$ should, however, ideally offer an etymology of pa-ra-ku-we and *56/pa-ra-ku-ja. The most commonly accepted interpretation is that these terms are related to alphabetic $(\sigma) \mu \alpha \alpha^{\prime} \alpha \gamma \delta \circ \varsigma$, 'emerald, blue turquoise' (probably a

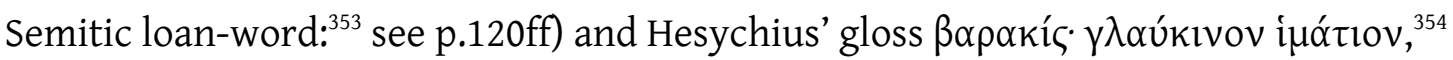
with a Mycenaean form /baraku-/ (Docs²:340; Melena 1987b); ${ }^{355}$ pa-ra-ku would therefore denote the material emerald/turquoise (or perhaps blue-green coloured glass) ${ }^{356}$ and *56/pa-ra-ku-ja a blue-green colour. Melena 1987:224-30 explains the alphabetic alternation between $\mu$ and $\beta$ as parallel to the Mycenaean alternation between ${ }^{*} 56, p a$, and $m a$ (and $\varnothing$, if tu-da-ra is taken as a shortened form of tu-*56-da-ro/tu-ma-da-ro) and as resulting from attempts to render an original Minoan phoneme / $\mathrm{m} b /$, which in Greek might naturally fluctuate between being interpreted as closest to $/ \mathrm{m} /$ or $/ \mathrm{b} /$ (with the

\footnotetext{
${ }^{352}$ The other possible alternations all involve incomplete terms or the comparison of only parts of terms as possible non-Greek onomastic elements, and are therefore excluded from discussion.

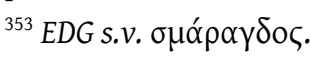

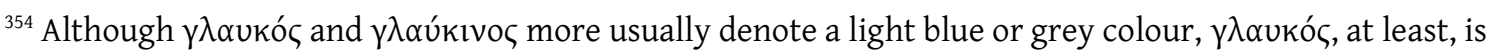
also attested referring to a more blue-green colour, including one instance in which it is applied to

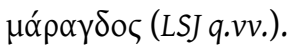

${ }^{355}$ There is little merit in the alternative suggestion that pa-ra-ku-we is connected to alphabetic $\beta p \alpha \chi u ́ c$ (Docs':340; L.R. Palmer 1955b), since it is hard to see what the meaning 'short' could have to do with a decorative precious material and a colour/decoration of textiles. ${ }^{356}$ Cf. p.116.
} 
$\varnothing$-alternation occurring in cases of syllable-final /-m/ preceding a consonant and therefore not being represented). This hypothesis has already been mentioned briefly relating to $p u_{2}(p .33, n .84)$ and ${ }^{*} 22$ (interpreted by Melena as $\left.p i_{2}: p .91\right)$, which were shown to offer it little or no support: ${ }^{*} 56$ is in fact the main basis for Melena's identification of this Minoan / ${ }^{\mathrm{b}} \mathrm{b} /$ phoneme. However, quite apart from the insecurity of an argument based on alternations which are by no means secure and a Hesychian gloss of unknown origin, this encounters two serious difficulties.

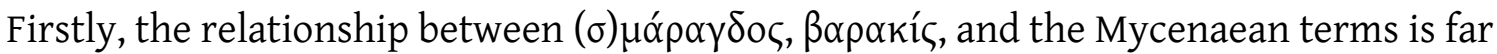

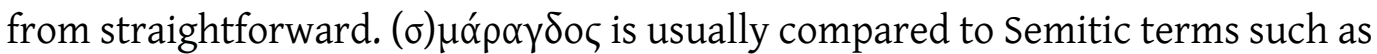
Akkadian barrāqtu, Hebrew bāreqet (*brq, 'shine'), although Piquero 2015:118-20, arguing that that the interpretation of these terms as specifically 'emerald' rather than more generally 'gem' is based chiefly on their identification with $(\sigma) \mu \alpha$ ó $\rho \gamma \delta \circ \varsigma$, so that using them as an argument for the latter's etymology is entirely circular, suggests an alternative Semitic derivation from *wrq, 'be green/yellow-coloured'. A Semitic origin

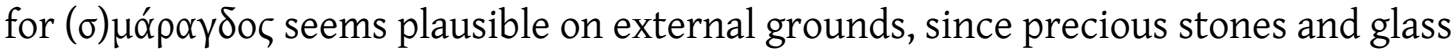
both often reached Greece from the Eastern Mediterranean; ${ }^{357}$ and it is certainly possible that such a word could have been borrowed into Mycenaean Greek, directly and/or via Minoan. However, neither etymology explains the origin of the initial $\sigma-$, which, far from being 'adventitious' (Melena 1987b:225), appears to be the earlier attested form; ${ }^{358}$ nor

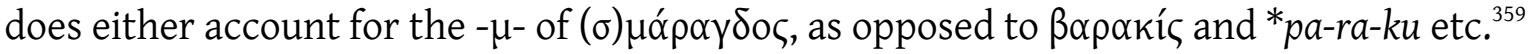

\footnotetext{
${ }^{357}$ See, e.g., Hughes-Brock 2011:99-102; Jackson-Wager 2011:116-17.

${ }^{358}$ LSJ q.v.; EDG suggests the possibility of influence from $\sigma \mu \alpha \rho \alpha \gamma \varepsilon \omega^{\omega}$ 'to crash', but this is hardly plausible on etymological grounds (cf. CDE q.v.).

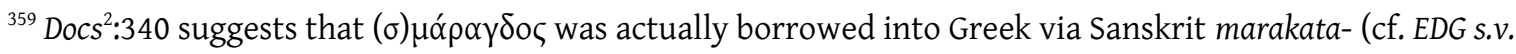
$\sigma \mu \alpha \alpha^{\rho} \alpha \gamma \delta$ $(\varsigma)$. A borrowing at different times from different languages could explain the $/ \mathrm{m} / \sim / \mathrm{b} /$ alternation, but on geographical grounds this seems rather implausible, and in any case the Sanskrit $m$ - is equally unexplained.
} 
In any case neither $/ \mathrm{b} /$ nor $/ \mathrm{w} /$ is likely to have been borrowed as $/{ }^{\mathrm{m}} \mathrm{b} /$ even if Minoan did have such a phoneme. With no convincing explanation of the history of $(\sigma) \mu \alpha \alpha^{\rho} \alpha \gamma \delta$ o and its possible relationship to * $p a-r a-k u$ - and/or $\beta \alpha \rho \alpha \kappa i ́ s$, this can offer no support to the identification of *pa-ra-ku- as /baraku-/.

In principle, the Mycenaean terms could still be compared to $\beta \alpha \rho \alpha$ ís, barrāqtu etc.; however, this would still rely on the second problematic assumption underlying this hypothesis - namely that a sign $\mathrm{pa}_{3}$ could stand for $/ \mathrm{ba} /$ as well as $/ \mathrm{p}^{\mathrm{h}} \mathrm{a} /$; the conclusion that a value in /b-/ as well as / $\mathrm{p}^{\mathrm{h}}-/$ is implausible for $p u_{2}$ (pp.29-31) applies equally to any other members of the same series. The initial consonant of $p a-r a-k u$ and ${ }^{*} 56 / p a-r a-k u-j a$ ought therefore to be $/ \mathrm{p}^{\mathrm{h}}-/$ - for which no good alphabetic parallels are available - or a non-Greek phoneme capable of being interpreted as (close to) $/ \mathrm{p}^{\mathrm{h}}-/{ }^{360}$ Possibilities for the latter would include, for instance, an aspirated voiced labial $/ \mathrm{b}^{\mathrm{h}}-/$ or a bilabial fricative / $\beta$-/ (cf. the similar explanations suggested by Thompson 2005 and Davis 2014:214-20 for the use of $p u_{2}$ in $d a-p u_{2}$-ri-to-jo: p.31). Neither of these is particularly

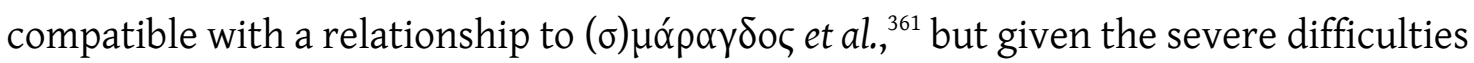

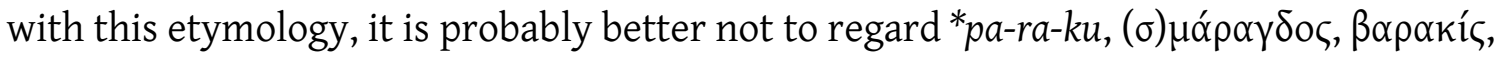
and the various Semitic terms as all being related to each other.

\footnotetext{
${ }^{360}$ The only proposed interpretation I am aware of which uses the value $/ \mathrm{p}^{\mathrm{h}} \mathrm{a} /$ for $* 56$ is the suggestion that

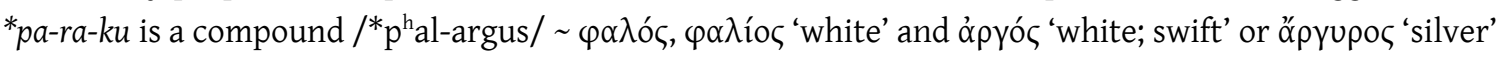
(Gallavotti 1957c:16-17). Such a compound, however, consisting of two adjectival members with very similar meanings, does not fit into normal Greek patterns of composition (on which see Meißner-Tribulato 2002:292-301): when á $\rho$ yó appears as the second member of a compound it has a nominal first member,

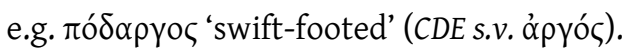

${ }^{361}$ Davis 2014:216 suggests that as Akkadian unemphatic stops had fricativised allophones, barrāqtu could have been pronounced with an initial [ $\beta]$, giving rise to its representation with a $p_{2}$-series sign in LA and hence in LB; however, a specific connection with Akkadian is far from certain.
} 
Ultimately, it is not currently possible either to prove or disprove whether $p u_{2}$ and other similar signs would originally have represented $/{ }^{\mathrm{m}} \mathrm{b} /, / \beta /$, or any other phoneme in Minoan, but what is more important for the purposes of this discussion is the way(s) in which sign ${ }^{5} 6$ is used within LB. Many of the terms in which it is attested are likely to be, or are plausibly, of Minoan/non-Greek origin, since most are PNs and TNs. It is therefore entirely possible that (a) non-Greek phoneme(s) could underlie some of these forms, and even that some attested examples of this sign could be attempts by LB scribes to render such a non-Greek phoneme in their writing of non-Greek names. In this context it may be worth noting that the only two vocabulary words in which *56 appears are found at Knossos, and could, therefore, plausibly be borrowings from Minoan, ${ }^{362}$ and that the sign is found in only four different PNs on the mainland. If $*_{56}$ does represent $/ \mathrm{p}^{\mathrm{h}} \mathrm{a} /$, this distribution might seem surprising, since such a sequence might be expected to appear within Greek vocabulary words, as $p u_{2}$ clearly does; ${ }^{363}$ why, for instance, is the word for 'cloths', / $\mathrm{p}^{\mathrm{h}}$ arwe(h)a/, never spelt with *56, despite this sign being used in other contexts by scribes who write textile records (e.g. H103, H116)? On the other hand, it is notable that $*_{56}$ is now found at both Pylos and Thebes: even supposing the names it is used to write to be non-Greek, the more important point is that the sign is in use at these sites at all. Regardless of its origin, ${ }^{*} 56$ is sufficiently integrated into the LB syllabary to be used at two different mainland sites as well as on Crete, and to appear overall about twice as frequently as $\mathrm{pu}_{2}$ : at this point it must surely have (in principle) represented a regular Mycenaean Greek sound value.

\footnotetext{
${ }^{362}$ Although the first part of ku-ru-su-*56 looks very like / $\mathrm{k}^{\mathrm{h}}$ rus-/ 'gold', the second part remains obscure, and this resemblance may be coincidental (cf. Melena 1987b:208-9).

${ }^{363}$ On the other hand, the root $/ \mathrm{p}^{\mathrm{h}} \mathrm{u}-/$, relating as it does to growing/plants, is perhaps one that is particularly likely to be represented within the LB tablets, given the concern (particularly at Pylos) with landholding; and it is of course possible that some of the PNs containing ${ }^{*} 6$ could in fact be Greek.
} 
Overall, given the plausibility of the value $p a_{3}=/ \mathrm{p}^{\mathrm{h}} \mathrm{a} /$ (both in general terms and specifically for an inherited sign with a distribution like that of $*_{56}$ ) and the existence of a reasonably secure alternation $p a-r a-k u-j a \sim{ }^{*} 56-r a-k u-j a$, it is very tempting to say that *56 should be regarded as 'deciphered'; the fact that this probably makes it necessary to reject the traditional interpretation of *pa-ra-ku etc. as /baraku-/ should not be a strong argument against accepting this identification, given the severe difficulties with this etymological identification discussed above. However, there still remain some slight caveats, particularly relating to the use of both *56-ra-ku-ja and pa-ra-ku-ja by the same scribal hand; until a better understanding of scribal variation renders H116's alternation between $p a$ and the proposed $p a_{3}$ unproblematic, and/or new discoveries of secure spelling alternations or Greek vocabulary words provide further corroboration, it seems better to err on the side of caution by maintaining *56's current undeciphered status.

\section{$2.8 * 63$ M}

*63 is found only on the mainland, with 17-20 certain/possible examples from Pylos and Thebes. ${ }^{364}$ The sign's basic structure consists of three verticals, joined by horizontals at the base and middle, with an uneven top. There are two main palaeographic variants: the most frequent form, found only at Thebes, has two triangular shapes at the top (the slight variation in the number and orientation of the strokes forming these shapes is not

\footnotetext{
${ }^{364}$ The suggestions that ${ }^{*} 63$ could be a variant of either *49 (Meriggi 1955:75) or pu (Georgiev 1956:76) are both unsupported by the palaeography of the signs in question; the latter has nothing in common with ${ }^{*} 63$, while the former has a similar basic structure but differs considerably in its details and formation. Plath 2004:11 compares *63 to the ideogram VIN 雨 ( $=$ AB131) on the grounds of their similar structures, based around three parallel vertical lines; however, AB131/vin differs significantly from *63 in several features, including its two horizontal strokes at the top of the sign, the small diagonal inner strokes, the lack of a base horizontal, and the occasional lack of the right-hand vertical, none of which has any parallels in examples of $* 63$. The differences are most clearly visible in the LB hands attested as writing both signs (PY H1; TH H306 and H308).
} 
palaeographically significant, since most of this variation occurs within a single hand, TH H306).

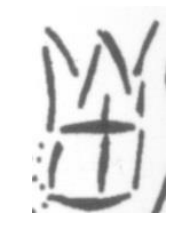

TH Fq 281.2 (H305)

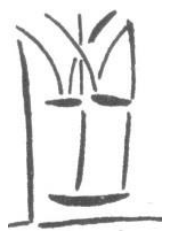

Gp $196.1(\mathrm{H} 306)$

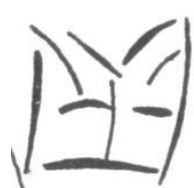

Gp $127.2(\mathrm{H} 306)$

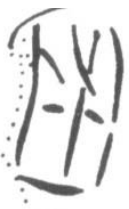

Gp $107.1(\mathrm{H} 306)^{365}$

Compare the more elaborate wavy top of TH H308 and PY H1:

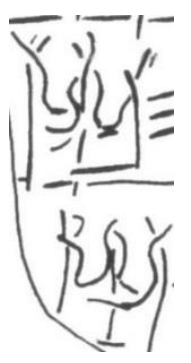

TH Gp 119.2.3 $(\mathrm{H} 308)^{366}$

\section{Imageremoved for \\ copyright reasons}

PY En 74.14 $(\mathrm{H} 1)^{367}$

The other example from Pylos, written by H41, appears midway between these two types, with a top that varies between a curved form similar to H1's (on the left) and a more triangular form similar to TH H305 (on the right).

\footnotetext{
${ }^{365}$ Drawings: $T F C^{3}$.

${ }^{366}$ Drawing: TFC ${ }^{3}$. This type is probably also found on TH Gf 163.3 (*63 [; unattributed). Note that $\mathrm{H} 308$ writes *63 with both a single central horizontal and a horizontal composed of two separate strokes; this therefore does not appear to be significant despite the difference between H305 and H306 in this respect. ${ }^{367}$ Photo: author (NAM).
} 


\title{
Imageremoved for \\ copyright reasons
}

\section{PY Eo $247.5(\mathrm{H} 41)^{368}$}

This implies that the first group of simpler forms could have arisen as a simplification of forms similar to the elaborate ones in $\mathrm{H} 1$ and $\mathrm{H} 308$, perhaps via an intermediate form similar to H41's.

The final possible example of ${ }^{*} 63$ at Pylos, on Vn 34.3, would if correctly read be a form similar to H1's; the reading *63-o-wa, by Godart and Olivier, is reported in Godart 2012:102.

\section{Imageremoved for copyright reasons}

\author{
PY Vn 34.3 (Ciii) $^{369}$
}

Although the left-hand part is certainly compatible with *63, there is no trace of the middle or right-hand vertical, even in the undamaged areas where these would be expected to appear; and although a large sign, ${ }^{*} 63$ is rarely if ever equivalent to twice the size of the neighbouring signs. The clearly visible stroke at the bottom right is also difficult to reconcile with *63 (unless read as a word-divider, which would produce a

\footnotetext{
${ }^{368}$ Photo: author (NAM).

${ }^{369}$ Photo: author (NAM).
} 
monosyllabic word). These traces are much more compatible with the reading -wi-(PTT; PoNIV), while the strokes to the left of the damaged area are almost identical in form to the -o- immediately following it, whose reading is not in dispute. I therefore regard the reading o-wi-o-wa as extremely probable, and do not include this as a plausible example of*63.

Likewise, the possible example on TH X 189.5 (H315) ([.]-u-ro-jo, possibly *6̣3-: TFC) seems better read as ka- (the other suggestion given by $T F C^{1}$ and $T F C^{3}$ as a less good possibility) since the remaining traces are rather more curved than would be expected for the righthand vertical of ${ }^{*} 63$, nor is it characteristic of this sign for the central horizontal to cross the vertical, as it clearly does on X $\mathbf{1 8 9 . 5}$.

Since there is no known LA equivalent to $* 63{ }^{370}$ and its attestation only on the mainland seems hard to ascribe to chance given the much larger number of tablets from Knossos than from any other site, ${ }^{*} 63$ was probably a mainland invention. Presumably this invention took place at a fairly early period - perhaps the point of the script's initial adoption on the mainland, or soon after - in order for this new sign to spread to more than one mainland site (in contrast to $r a_{3}$ and two, apparently invented at Pylos and found only at that site).

${ }^{370}$ On the improbability of a connection to AB131/vIN, see p.123, n.364. 


\section{Prospects of decipherment}

*63 is only certainly attested in three different terms - ku-*63-so (MN, Pylos), *63-u-ro (recipient at Thebes, probably an MN), ${ }^{371}$ and *63-te-ra-de (TN at Thebes in the allative, probably the name or location of a sanctuary to which offerings of wine are sent) ${ }^{372}-$ none of which has any good potential spelling alternations. ${ }^{373}$ Although the majority of this sign's attestations are word-initial, this is largely due to the frequent repetition of *63-te-ra-de throughout the TH Gp-series, as well as three probable attestations of *63-u-ro (and two more possible word-initial examples at Thebes which could belong to either of these terms); a word-initial appearance in two out of three different terms is not a high enough proportion to make a particularly strong case for a vocalic value, but equally not low enough to rule this out. The following - $u$ - in *63-u-ro suggests that a value in $-u$ is probably the least likely, and one in $-a,-e$, or -o (forming a diphthong) perhaps more likely than $-i$, but this is far from conclusive.

The suggestion that *63 =ji/zi (Melena 2014a:79-83 and forthcoming:31-4), ${ }^{374}$ based primarily on the hypothesis that $* 65=j u / z u$ (Melena 2014a:75-9, 81-3 and forthcoming:36-8), is implausible both in principle (since it involves the use of a single sign for three different $C V$ sequences, with a sign for */yi/ also coming to represent /dzi/ and /hi/ when */y/ > /dz/ or /h/ in some environments: cf. further discussion on

\footnotetext{
${ }^{371} \mathrm{TFC}^{1}: 218$. The possible example of this $\mathrm{MN}$ in the genitive is unlikely: see above.

${ }^{372} T^{1} C^{1}: 277$; Killen 2006:87-8.

${ }^{373}$ Proposals such as $r u_{2}$ (Georgiev 1956:76), nwi (Owens 1991-1993:265), or kra (Plath 2004) are generally based on completely insecure comparisons with other LB terms, quite apart from being types of values which would either not be expected at all, or not for a sign invented in LB (pp.64-5). The reasoning behind the suggestion $d w i$ (Ruipérez-Melena 1996:85) was never published, but in any case Melena 2014a:80 retracts this since *63-te-ra-de occurs on TH Gp 109.1 alongside di-wi-ja-me-ro, probably /dwi-āmeron/ '(for) two days' (Killen 2006:98; Hiller 2011:192).

${ }^{374}$ On the similar suggestion concerning ${ }^{*} 47$, see p.103, n.296.
} 
*65, p.144ff) and for this particular sign: a value zi is highly improbable and ji almost impossible for a sign invented in LB rather than inherited (pp.10-13), nor are the possible spelling alternations cited in support of this hypothesis remotely compelling. ${ }^{375}$ *63's probable LB invention makes values in $n w$ - or $p_{2}$ - similarly unlikely, while its distribution suggests it is probably not palatalised; but there is no real way of narrowing down the various remaining possibilities ( $i$-diphthongs, labialised consonants, $C t$ - clusters...). It is notable that ${ }^{*} 63$ does not display the kind of distribution seen for most of the extra signs invented in LB, as discussed in Chapter 1, being found only in proper nouns which may or may not be of Greek derivation; this might argue against a value such as a labialised consonant or a Ct-cluster, whose use seems to be primarily in the context of Greek vocabulary words and morphological formations, but this could equally be due to a variety of other factors (chances of attestation? a process of creation similar to that of two [p.42], but which for some reason led to a more widespread adoption?). In the end, it is not impossible that *AB63 is simply not yet attested - which would, of course, open up a range of other possible values for this sign. Given the current state of the evidence, this sign's prospects of decipherment are minimal.

\section{$\underline{2.9 * 64}[\mathbf{X}$}

*64 is a comparatively rare sign, with 10 examples at Knossos and Pylos; ${ }^{376}$ its only significant form of variation is the presence or absence of a small horizontal stroke at each end of the verticals. Both are these forms are seen at Pylos (the form with these

\footnotetext{
$375 * 63-u-r o$ is compared to ki-u-ro (an MN at Knossos) and both are suggested to begin /Ski-/ based on the occasional alternation between ze and ke for /ske-/ (on which see Killen 1979:164-8); ku-*63-so is compared to the incomplete ]ku-i-so, also an MN at Knossos.

${ }^{376}$ There is no palaeographic basis for the suggestion that ${ }^{*} 64$ is a variant of ${ }_{56}$ (Georgiev 1956:80), since the former's central diagonal cross clearly distinguishes it from all the other LB signs based on two verticals with varying numbers and types of strokes between them.
} 
strokes in H1, H2, H21, and H31/27; the form without in H23 and Ciii), while only the first is found at Knossos; as there are only two Knossian examples compared to eight from Pylos, it is not possible to say whether this is due to chance or to an actual palaeographic difference between the two sites.

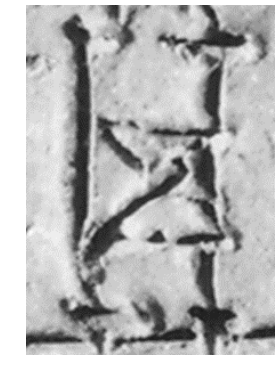

PY Jn 832.11 (H2)

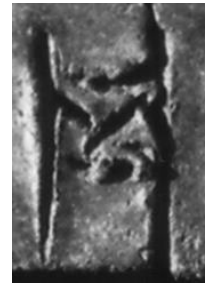

PY Vn $34.2($ Ciii) 377

Variation in the proportions of the central square relative to the overall size of the sign do not appear to be significant, since this can vary even within a single hand, as seen in the two H23 examples below.

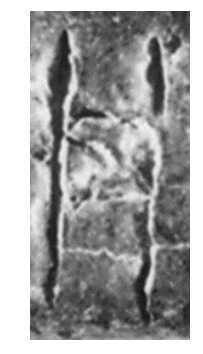

PY Ad 326

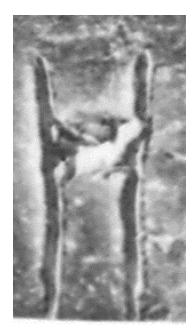

PY Ad $315^{378}$

*64 has no generally accepted LA correspondence, although it has been compared to AB164 (L43), ${ }^{379}$ whose form $\bigotimes$ resembles the central element of *64. However, this form appears only once (HT 17.1), 6 being the more frequent form; neither ever appears with

\footnotetext{
377 Photos: MER.

${ }^{378}$ Photos: MER.

${ }^{379}$ See, e.g., Packard 1974:104; Raison-Pope 1977:60; Meijer 1982:40.
} 
the extended verticals characteristic of ${ }^{*} 64$. AB164 therefore resembles much more closely the LB ideogram *164, 捣 or (probably a kind of cloth: Docs²:51), and it is better to see $* 4$ as probably a new creation in LB (although of course a chance absence of *AB64 is always possible).

Melena forthcoming:35 describes *64 as 'a Mainland tradition abandoned early at Knossos' on the grounds that, of the two Knossian examples, Sc 261 is certainly from the RCT and X 5516 is probably also from this location. ${ }^{380}$ The latter is, however, an unattributed tablet belonging to a batch of fragments which are associated by scribal hand with a range of locations in the western, southern, and northern parts of the palace $;^{381}$ no other tablet from this batch is attributed to the RCT. Although an origin in either the RCT or another early deposit cannot be ruled out, ${ }^{382}$ there is no strong reason to assign X 5516 an early date in this way; nor does the probable attestation of the same MN on this tablet and Sc 261 form an argument for dating them to the same period, since there may well be two or three contemporaries with the name $a-{ }^{*} 64-j o$ at Pylos. ${ }^{383}$ It therefore seems most probable that ${ }^{*} 64$ remained in use, though as a very rare sign, at Knossos as at Pylos.

\footnotetext{
${ }^{380}$ The only possible exception is the reading $a-z e-t i{ }^{*} 64-j a$ on KN Ln 1568 lat.inf. .a (suggestion by G. Huxley, reported by Chadwick 1957 [unpublished]); this has never been accepted (the term is generally read $a$-ze-tiri-ja, cf. other examples of this term at Knossos) and appears highly improbable based on autopsy of the tablet; this is therefore excluded here.

${ }^{381}$ Firth-Melena 2000-2001:322-3, 345.

${ }^{382} \mathrm{H} 5$, to which two of the fragments from this batch are assigned, has been argued to be of earlier date than the other West Wing tablets (e.g. Firth 2000-2001b:200-1) but this has been challenged on archaeological grounds (e.g. Hood 1965:36).

${ }^{383}$ It is possible that $a^{-}{ }^{*} 64-j o$ on Jn 832.11 and Cn 1287.1 refer to the same person, but Nakassis 2013:219 regards these and the third example on Fn $\mathbf{3 2 4 . 3}$ as probably different individuals.
} 


\section{Prospects of decipherment}

All of the examples of 64 from both sites appear in forms of the same word, apparently an ethnic adjective also used as a PN: $a^{-}{ }^{*} 64-j o\left(M N\right.$, nom. or dat.), $a^{-*} 64-j a$ (WN, f.nom.sg.; appellative, f.nom.pl.), and $a^{*}{ }^{*} 64-j a-o$ (appellative, f.gen.pl.). ${ }^{384}$ This means that there is little information to go on in order to attempt a decipherment of this sign. ${ }^{385}$ The fact that *64 is preceded by $a$ - suggests that a $C(C) V$ structure may be more likely than a vocalic sign; the fact that it is followed by $-j$ - is not necessarily significant in suggesting a value in -i-, since this could equally represent the adjectival suffix /-ios, -ia/ following a different vowel. In principle, therefore, almost any of the values argued in Chapter 1 to be the most plausible for a sign invented in LB are possible.

In practice, however, ${ }^{*} 64$, like ${ }^{*} 63$, does not display the kind of distribution seen in many of the newly-created extra signs; its use in only a single ethnic adjective/PN could be due to chance, as could its lack of an LA correspondence, but if this is not the case, it would imply a more sporadically useful sound-value, perhaps created specifically for use in this term and used by a relatively wide range of scribes only due to the frequent recurrence of this ethnic/PN. If this is the case, then this distribution could be compatible with, e.g., a palatalised sign representing the clusters /wy/ or secondary /sy/ or /py/, which (if they occurred at all) would be primarily expected in this kind of adjectival formation (pp.60-1); the relative frequency of wi-j- and si-j- for /wy/ and secondary /sy/ might argue, not against the existence of a complex sign for either of these clusters, but against one with such a restricted use, leaving secondary /py/ as perhaps the best of these

\footnotetext{
${ }^{384}$ The restoration $\left.a-\right]^{*} 64$-jo on $\mathrm{KN}$ X 5516 seems secure.

${ }^{385}$ Most of the various suggested values, including $n w i$ (Gallavotti 1956a:24), su $\left(\right.$ Packard 1974:104), $r e_{2} / \mathrm{ri}_{2}$ (Chadwick 1957 [unpublished]; Tritsch 1958:430-1, n.48, and 443-5, n.72) and zi (Witczak 1999), are both unlikely (either in general or for a sign invented in LB) and based on insecure comparisons with other LB or LA terms and/or on misreadings of LB texts.
} 
options. Alternatively, secondary /sw/ has similarly been shown to occur only sporadically, and primarily in ethnics and PNs, in contrast to other / $\mathrm{Cw} /$ clusters which occur in more morphologically useful contexts (pp.60-1); since this possibility fits in with the most common proposal for *64's sound-value, swi, ${ }^{386}$ this is worth examining in greater detail.

The proposal that ${ }^{*} 64=$ swi is based on the identification of $a^{-}{ }^{*} 64-j 0 /-j a$ with the similar PN/adjective $a$-si-wi-jo/-ja. ${ }^{387}$ The interpretation of the latter as an ethnic /Aswios, $-\bar{a} /$, related to classical 'Ă oí $\alpha$, is plausible enough phonetically, since alternations in the length of the first vowel (cf. Hom. 'Āoíw), along with the Hittite Aššuwa (referring to an area in western Asia Minor, as 'A бí $\alpha$ seems originally to have done) ${ }^{388}$ suggest an original form */Aswia/ (Chadwick 1968:63); however, it is unclear whether po-ti-ni-ja a-si-wi-ja on Fr 1206 refers to an offering of oil being sent to Asia Minor, ${ }^{389}$ to a goddess called 'Asian' but worshipped in Messenia, ${ }^{390}$ or (conceivably) to a place in Messenia called /Aswios/ (vel sim.). The interpretation of $a^{-*} 64-j o /-j a$ as specifically an ethnic adjective is also plausible, given the appearance of other ethnics describing groups of women in the PY Aa- and Ab-series, and the fact that some of these other ethnics appear to refer to coastal areas of Asia Minor (mi-ra-ti-ja = /Milatiai/ 'women from Miletos'; ra-mi-ni-ja = /Lamniai/ 'women from Lemnos'; ki-ni-di-ja = /Knidiai/ 'women from Knidos': Chadwick 1988:78-84) makes the interpretation 'Asian' (probably referring to Lydia) entirely possible - though, again, a place within Messenia cannot be ruled out, since other locations are also found

\footnotetext{
${ }^{386}$ Gallavotti 1958c:2; Chadwick 1968a:63-4.

${ }^{387}$ a-si-wi-jo (MN): KN Df 1469 (H117); PY Cn 285.12, Eq 146.11 (H1); MY Au 653.5, 657.11 (H62). a-si-wi-ja

(f.nom.sg. adjective): PY Fr 1206 (H2).

${ }^{388}$ Morris 2001:425-6.

${ }^{389}$ Bendall 2014:147-8.

${ }^{390}$ E.g. Morris 2001.
} 
(e.g. ku-te-ra $a_{3}=/$ Kut ${ }^{\mathrm{h}} \mathrm{e} r \mathrm{rai} /$ ' $w o m e n$ from Kythera'; $t i-n w a-s i-j a=$ 'women from *ti-nwa-to', a place apparently within Pylos' territory, perhaps in the Further Province). ${ }^{391}$ Whichever interpretation of these two ethnics is adopted, however, there is no particular evidence to show whether the location referred to is the same, nor is there any evidence linking any of the instances of $a-s i-w i-j o$ to those of $a-{ }^{*} 64-j o$ as referring to the same individual. The former appears as a landholder on Eq 146.11 and in a list of people at ro-u-so who are responsible for livestock on Cn 285.12, probably referring to two different people; neither of these has any apparent prosopographical connection to the smith on Jn 832.11, the recipient of HORD on Fn 324.3, or the 'messenger' contributing a goat on Cn 1287.1 (Nakassis 2013:213-4, 219).

More problematically, as Melena forthcoming:36 points out, almost every hand attested as using *64 is also attested as writing the sequence si-wi in scriptio plena: $\mathrm{H} 1$ and $\mathrm{H} 2$ actually do so in the term $a$-si-wi-jo/-ja (p.132, n.387), while H21 and H23 (as well as H1) have examples of another ethnic, ki-si-wi-ja(-o), ${ }^{392}$ perhaps /Kswiai, -āhōn/ 'from Khios, ${ }^{393}$ It is particularly difficult to explain why $\mathrm{H} 1$ and $\mathrm{H} 2$ should use both $a-s i-w i-j o /-j a$ and $a-{ }^{*} 64-j o /-j a$ if these are in fact variant spellings of the same term; although alternations between a complex sign and scriptio plena in the same word are not unheard of (e.g. PY H26 writes both te-mi-dwe-ta and te-mi-de-we-te, ${ }^{394} \mathrm{H} 41$ writes both $d w o$ 'two' and $d u-w o-u-p i),{ }^{395}$ neither do they seem to be particularly common (Panayotou 1992 claims that scribes very frequently alternate between complex signs and scriptio plena, but gives no figures on how often this occurs in the same word; a full study of

\footnotetext{
${ }^{391}$ Chadwick 1988:78-84.

${ }^{392}$ PY Aa 770 (H1); Ab 194.B (H21); Ad 675 (H23).

${ }^{393}$ Chadwick 1988:80.

${ }^{394}$ Sa 791, 793; Sa 1266.a.

${ }^{395}$ Eb 338.B, Eo 278; Eb 149.2, 495.1.
} 
scribal usage of all the complex signs in this regard is beyond the scope of this thesis). The suggestion that *64 therefore represents twi, with $a^{*} 64-j o /-j a$ being a conservative spelling preserved in the ethnic adjective (Melena forthcoming:35-6), is linguistically implausible (although, as previously shown, there is no evidence as to whether the sound-change affecting */tw/ had begun in Mycenaean Greek, ${ }^{396} / \mathrm{sw} /$ is unlikely to have been the outcome of this change at any stage $)^{397}$ and would in any case not solve the problem of scribal variation, since we would still be left with individual hands using both the 'conservative' and the 'progressive' spelling. Nor is twi a particularly plausible value for any LB sign, whether inherited or invented (p.44ff).

Although the value swi cannot be ruled out for *64, the equation of $a^{*} 64-j o /-j a$ to $a$-si-wi-jo/-ja is therefore somewhat less secure than is often assumed. In the absence of attestations in terms other than $a^{-*} 64-j o /-j a$, or an actual alternation of these two terms in the same context, it cannot be regarded as certain, and the question of this sign's value remains open.

\section{$\underline{2.10 * 65}$ ऋ}

*65 is a relatively frequent sign: along with the morphologically identical ideogram FAR 'flour ${ }^{\text {’38 }}$ it has 59-71 certain/possible attestations at Knossos, Pylos, Mycenae, and

\footnotetext{
${ }^{396}$ See p.39.

${ }^{397} \mathrm{As} * / \mathrm{tw} /$ has the same outcomes in classical Greek as */ty/ and */ky/ ( $\sigma$ - initially, $-\sigma \sigma-/-\tau \tau-$ medially) it is likely to have gone through a process similar to the palatalisations of these clusters, with an affricate */t $\mathrm{t}^{\mathrm{s}} /$ vel sim. as the intermediate stage (Lejeune 1972f:82-3, 105-11).

${ }^{398}$ This interpretation is based on FAR's occurrence on PY Un 718.10 after the term me-re-u-ro /meleuron/

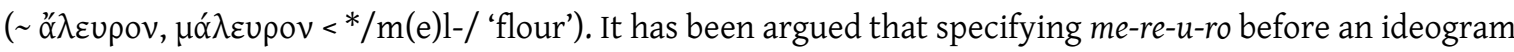
meaning 'flour' would be redundant and that therefore FAR instead represents a type of grain (R. Palmer 1992:488-90; Halstead 1995:232; Duhoux 2008:346), distinct from both wheat and barley (which are
} 
Thebes. Only 23-28 of these examples, however, are certainly syllabographic, with 18-20 certainly being ideographic; it is controversial whether the examples at Thebes represent this sign's syllabographic or ideographic use or a combination of the two. ${ }^{399}$ Since this issue has the potential to double the number of syllabographic instances of *65/FAR, and therefore to make a significant contribution towards a potential decipherment (not to mention its wider implications for issues such as the possible religious interpretation of the Thebes tablets $)^{400}$ it will be discussed at some length below.

The essential characteristics of *65/FAR are a vertical line with a diagonal or horizontal attached to its right-hand side, with two or three shorter verticals placed under this diagonal. This sign's variant forms can be divided into two main groups - Type 1 (more elaborate forms) and Type 2 (simplified forms) - although within each of these groups there is still a considerable amount of variation. ${ }^{401}$

Type 1 is in general characterised by a vertical or T-shape which crosses the main diagonal/horizontal; within this group there is variation as to the form of this part of the

represented by GRA and HORD, or perhaps vice versa: for a range of opinions on this debate see R. Palmer 1992 and 2008; Halstead 1995; Killen 2004). However, as on Un $718 \mathrm{H} 24$ also writes tu-ro $\mathrm{r}_{2}$ before $T U+\mathrm{RO}_{2}$ and a-re-<pa $>$ before $A+R E+P A$, this 'redundancy' is unproblematic, and FAR is plausibly equivalent to me-re-u-ro. It is, however, not certain whether, like the related classical terms, me-re-u-ro and/or FAR meant specifically 'wheat flour', or whether this could refer to any milled grain, though the attestation of FAR alongside both HORD and GRA makes the latter perhaps more plausible (Killen 2004:167-8; R. Palmer 2008:637).

${ }^{399}$ In general, even scribes who have written both *65 and FAR (KN H139; PY H1, and perhaps H2) make no palaeographic distinction between the two. The only exception to this is also the only instance of both appearing on a single tablet, KN Fs 3 (-), on which they clearly have different forms (see pp.136-7). ${ }^{400}$ Since FAR appears to be attested only in religious contexts at other sites: Killen 2006:85.

${ }^{401}$ On the palaeography of *65/FAR, see also Raison 1964:60 (Fig. 1); my analysis of these forms, however, differs from his four-way division. 
sign (diagonal, curve with branching end, two wavy lines) as well as in the position and proportion of the various elements, the presence or absence of horizontals at the top and/or bottom of the short vertical strokes, etc., as shown by the images below. ${ }^{402}$

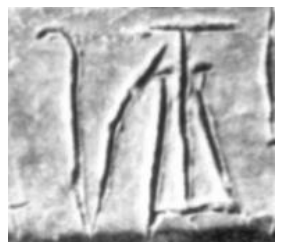

Type 1: KN U(1) 49 (H124-E)

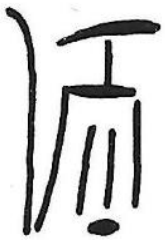

$\mathrm{KN} \operatorname{Od}(2)$ 714.a (H103)
Image

removed for

copyright

reasons

PY Un $853.5(\mathrm{H} 6)$

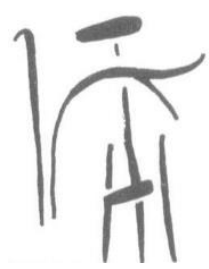

Imageremoved

for copyright

reasons

PY Jn $725.8(\mathrm{H} 2)^{403}$

This is the most common form at Knossos (seen in H103, H117, H124-E, H139, and on X 9519), and is also seen in around half the examples at Pylos ( $\mathrm{H} 1, \mathrm{H} 2, \mathrm{H} 6$, perhaps $\mathrm{H} 42,{ }^{404}$ and the Class ii hand of Vn 46). Note the palaeographic differences between the two sites - the H6 example is the only instance at Pylos of a form similar to the usual Knossian one, while the two wavy lines of the more usual Pylian form have no exact parallel at Knossos. Note also that both examples of *65/FAR on KN Fs 3 fit into this group, despite their significant differences from each other: the ideographic form (green) is similar to

\footnotetext{
${ }^{402}$ Some of these elements may vary even within a single hand: for instance, H139 sometimes includes a small horizontal, but often does not, and the diagonal may be curved or straight.

${ }^{403} \mathrm{KN}$ photo: MER; KN drawings: COMIK; PY photos: author (NAM).

${ }^{404} \mathrm{H} 42$ has only an uncertain example of [[FAR]] (Un 47.4), but if this reading is correct the traces are more compatible with this form than the simplified one.
} 
that seen in H139 (above), while the syllabographic form (red) is unique in its use of short diagonal strokes to the side of the main vertical.

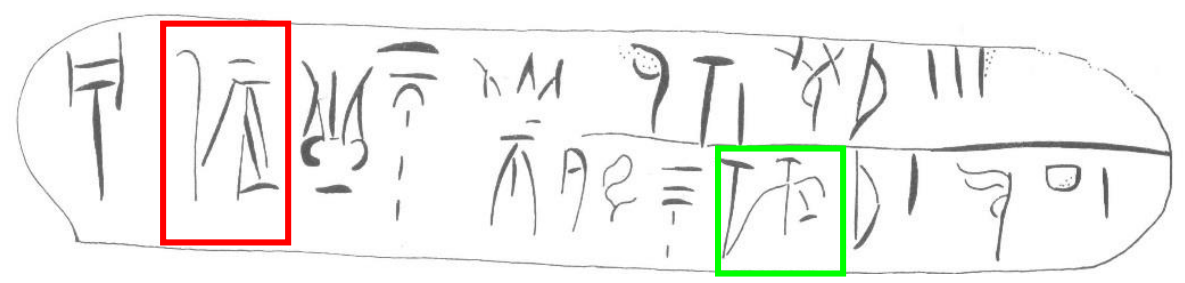

$\mathrm{KN} F s 3^{405}$

Type 2 is characterised by a diagonal with one or two vertical strokes under (but not usually crossing) it, sometimes with a base horizontal.
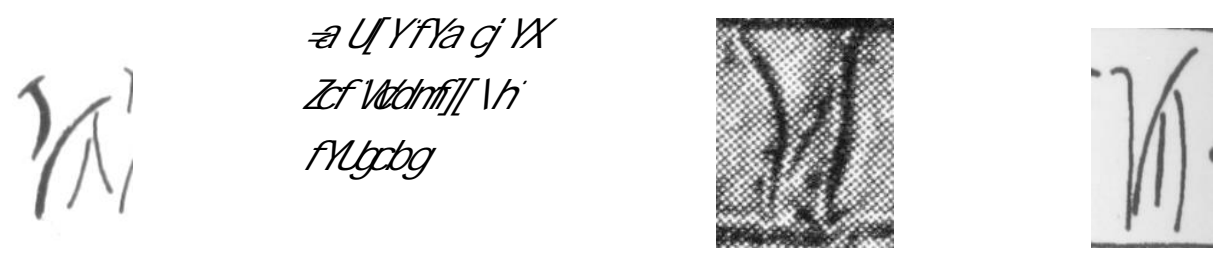

Type 2: KN Xd 105 (H124-4); PY Eo 276.8 (H41); MY Fu 711.7.8 (-); TH Fq 258.5 (H305)
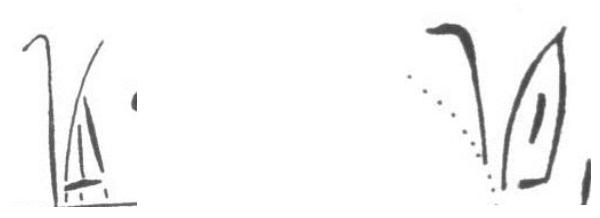

TH Gp 215.2 (-); TH Gp $110.2\left(\right.$ H306) ${ }^{406}$

Again, as can be seen above, there is some variation in the positioning and proportions of the component strokes, particularly in the relative lengths of the verticals, and

\footnotetext{
${ }^{405}$ Drawing: COMIK. The use of these different forms suggests that this scribe, at least, thought of $* 6$ and FAR as distinct signs (Killen 2006:103, n.72), though whether they habitually used both of these forms or whether the distinction between them was occasioned by their unusual appearance on the same tablet is unknown.

${ }^{406}$ KN drawing: COMIK. PY photo: author (NAM). MY photo: SCM. TH drawings: TFC ${ }^{3}$.
} 
whether the right-hand one meets the middle or the end of the diagonal. ${ }^{407}$ This form occurs only once at Knossos (in the RCT), but accounts for around half the examples of *65/FAR at Pylos (H21, H24, H41, and the Class ii hand of Vn 879) and two of the three examples at Mycenae, and is the only form found at Thebes. ${ }^{408}$

There is, in addition, a (rare) intermediate form which combines elements of both types: this is in most respects similar to the simplified form, but like the first group its vertical crosses the diagonal.

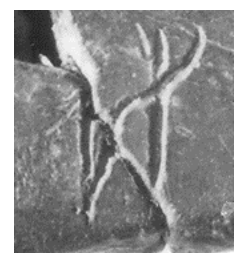

PY Ae $344(\mathrm{H} 22)^{409}$

*65's LA antecedent, AB65, is relatively well attested, with 24-37 examples in GORILA; ;10 $^{40}$ the majority of these are syllabographic, but with up to four possible ideographic examples of $A B 65,{ }^{411}$ it seems plausible that ${ }^{*} 65 /$ FAR's dual function may have originated in LA. Most forms of AB65 are very simple: a vertical with a diagonal line or curve up to the right, with a vertical stroke crossing this diagonal or positioned at its end. Some

\footnotetext{
${ }^{407}$ Again, these features can vary even within a single scribal hand: e.g. H306's other examples have a longer central stroke; $\mathrm{H} 305$ has some examples with a lower horizontal (e.g. Fq 342.2).

${ }^{408}$ As the question of whether these Theban examples are ${ }^{*} 65$ or FAR is not purely a palaeographic one, discussion of this will be postponed until the 'prospects of decipherment' section.

${ }^{409}$ Photo: MER; cf. the similar example on MY Au 653.6 (H62).

${ }^{410}$ There is no known $\mathrm{CH}$ equivalent to $\mathrm{AB} 65$; the suggestion that $\mathrm{CH} 061$ and $\mathrm{AB} 65$ may have had similar sound-values (Younger 1996-1997:387, 397) is not made on a morphological basis.

${ }^{411}$ The examples on HT 97a.4-5 (isolated signs) and HT 20.2.4 (both at the ends of sign-sequences, followed by ideograms) could be either ideographic (Younger (LA HT)) or syllabographic (GORILA); the possible examples on ZA 11a.4 (followed by a fraction sign) and HT $\mathbf{8 9 . 2 ~ ( i n ~ a ~ l i g a t u r e ) ~ w o u l d ~ b e ~ i d e o g r a p h i c ~ i f ~}$ correctly read.
} 
variants have elements added underneath the diagonal, usually a small stroke and/or a circle/dot. ${ }^{412}$

\section{Imageremoved for copyright reasons}

There are no close LA parallels for LB Type 1; cf. the fact that the only example of the simpler form from Knossos comes from the RCT, which is probably significantly earlier than the rest of the Knossos tablets. ${ }^{413}$ It appears, therefore, that this simpler form may have been the original form, with the more elaborate forms being a subsequent development, either in late LA or in early LB - though it is also possible that the elaborate LB forms could have originated from a similar LA prototype which is not attested in the extant records.

\section{Prospects of decipherment}

The majority of attestations of *65/FAR are in PNs or TNs or are ideographic, meaning that they are of limited use in assigning a sound-value. Likewise, the debate over whether the Theban examples of this sign are syllabographic or ideographic means that they also cannot be securely used in an attempt at decipherment; these will be analysed after the discussion of *65's possible value based on its secure attestations.

\footnotetext{
${ }^{412}$ Drawings: GORILA. The PA 1 form, with its three strokes above the diagonal, appears closer to LB *161 $\mathrm{p} \nVdash$ (which has no certain LA antecedent) than to *65/FAR.

${ }^{413}$ See p.233.
} 
The syllabogram is attested in only three certain vocabulary words, $i-{ }^{*} 65$ (an appellative - see below), pe-*65-ka, and e-pi-*65-ko (both nouns, probably construction materials). Despite this, ${ }^{*} 65$ is the undeciphered sign most widely regarded as having a known sound-value, to the extent that it frequently appears transcribed with this (tentative) value, $j u^{?}$ (as first proposed by L.R. Palmer 1955b:43 and Meriggi 1955:76). ${ }^{414}$ Its 'undeciphered' status has, however, been maintained since the 1965 decision of CIPEM not to officially adopt this transliteration. ${ }^{415}$

ju was previously identified (p.11) as being the most likely of the 'missing' core values to be found amongst the undeciphered signs; ${ }^{*} 65$ also fulfils the criteria of being inherited from LA and appearing with a relatively high frequency after front vowels, particularly /i/, ${ }^{416}$ both as expected for a sign in $j$-. The strongest evidence for this value, however, comes from one of the three vocabulary words in which it is attested, $i-{ }^{*} 65$, which occurs up to five times at Pylos. This word occurs in the same contexts as PNs, is listed as a single (male) person in three cases (Ae 344; Jn 431.6, 725.8), and always appears in connection with another appellative, whether this is an MN in the genitive (Aq 64.7, 218.16), an adjective in /-ios/ in the nominative (Ae 344), or an $\mathrm{MN}$ in the nominative (in which case it appears as $\left.i^{-*} 65-q e: \mathrm{Jn} 431.6,725.8\right)$. It is clear that $i-^{*} 65$ a) refers to a single person and b) expresses a kind of relationship between individuals; since the obvious candidate for a relationship which can be indicated by a genitive, by an adjective in

\footnotetext{
${ }^{414}$ Alternative suggestions such as ní (Georgiev 1956:67-8, based on Lurja 1955) and ja $a_{2}$ (Landau 1958:13) are based on highly insecure comparisons with other LB and/or classical Greek terms and have not met with general support.

${ }^{415}$ L.R. Palmer-Chadwick 1966:7-8; this decision was reaffirmed in 2000 and 2010 (Olivier 2012a:581).

${ }^{416}$ L.R. Palmer 1955b:43. There are 13 different terms in which *65 is certainly attested and has a securelyread preceding sign; 8 of these (62\%) follow a front vowel, which in 6 cases ( $46 \%$ of the total) is /i/. These figures do not include any examples from Thebes, due to their uncertain status; however, it is worth noting that the majority of possible Theban examples follow a back vowel. This may be due to the particular circumstances of this sign's use at Thebes, which will be addressed below.
} 
/-ios/, or by the phrase ' $\mathrm{X}$ and $\mathrm{Y}$ ' is a parent/child one, the meaning 'son' is generally accepted for $i_{-}^{*} 65$, which is therefore interpreted as $i-j u /(h) \bar{y} y u s / \sim$ vió $\zeta<* /$ sūyus $/{ }^{417}$

The spelling in $i$ - is confirmed by the entry di-ri-mi-jo, di-wo , $i$-je-we on PY Tn $316 v .10$, in which $i-j e-w e$ is most plausibly a dative /(h)iyewei/, the whole entry meaning 'to D., son of Zeus' ${ }^{418}$ However, the sequence -u-jo in the entry ra-]ke-da-mo-ni-jo-u-jo on TH Gp 227.2 is equally plausibly a form of /(h)ūios/ vió ${ }^{419} .{ }^{419}$ The existence of these two different forms is not necessarily problematic, ${ }^{420}$ particularly as they are attested at different sites. A wide variety of forms are attested in alphabetic Greek alongside viós, including huóৎ, huiv́s, huv́s, húc, hữí, and fhió ${ }^{421}$ the differences between them all being explicable as the results of different dissimilatory changes operating on */sūyus/ > */hūyus/ (and in some cases then $>* /$ hüus/): either a change of one of the two /u/ vowels to /i/, or creation of an o-stem form in /-os/, with a similar dissimilatory effect. ${ }^{422}$ This situation seems to be exactly paralleled in Mycenaean, with an o-stem form u-jo /(h)ūino-/ at Thebes and a dissimilated form $i^{*} 65$ /(h)ìyu-/ at Pylos. ${ }^{423}$

\footnotetext{
${ }^{417}$ See, e.g., Ruijgh 1967:361-2; CDE s.v. vióc; Risch-Hajnal 2006:193, 228. On the phonemic interpretation of this term - particularly the status of the $/ y /$, and whether this is in fact still phonemic at this point - see pp.156-7; in the meantime, I continue to refer to this term as /(h)iyus/ since this must at least have been its original form.

${ }^{418}$ Ruijgh 1967:362. Docs ${ }^{1}: 286$ suggests that $i$-je-we is an error for $i-j e-<r e->w e ; D^{2} c^{2}: 172$, however, prefers the interpretation 'son' to 'priest', and (pace interpretations of Tn 316 as 'carelessly written', e.g. Docs':172) there seems no particularly good reason to assume a scribal error here; the idiosyncrasies of this text have been variously explained as due to the scribe experimenting with the layout of this complicated document (Palaima 1999:444-50) or as being due to the involvement of two different scribes (Godart 2009). ${ }^{419} \mathrm{TFC}^{1}: 396$. On this entry, see further pp.153-4.

${ }^{420}$ The suggestion that $i$ - is a purely graphic convention to render /(h)ūi-/ (Lejeune 1971b) does not seem a particularly plausible one, since this sequence can be represented perfectly effectively by $u-j-$.

${ }^{421}$ Duhoux 2008:355.

${ }^{422}$ Duhoux 2008:355.

${ }^{423}$ Two other terms have also been less plausibly suggested to be forms of this word. $i$-we (KN Nc 8176) could be another dative form /(h)iwei/, but the tablet is too fragmentary to be certain (Ruijgh 1967:362).
} 
Possible corroboration for the value ju might come from the alternation of $r{ }^{-}{ }^{*} 65-n o$ (KN $\mathrm{U}(1) 49)^{424} \sim$ ri-u-no $(\mathrm{KN} \mathrm{Xd} \mathrm{149)} \sim$ the TN ri-jo-no (at least 24 examples in the KN Ap-, C-, and D-series), if these are correctly identified as the same TN. As the single occurrence of ri-u-no follows the TNs e-ra and tu-ni-ja, and o/u alternations are not uncommon (in either LB or alphabetic Greek) in words derived from non-Greek languages, ${ }^{425}$ it seems most likely that this term is both a TN and the same location as ri-jo-no; however,

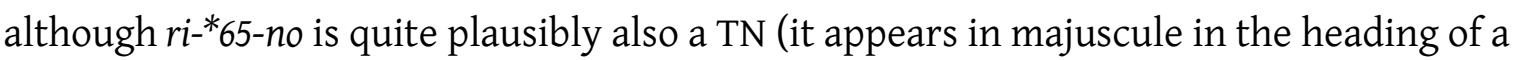
palm-leaf tablet), and a further alternative spelling ri-ju-no would be unproblematic (for LB alternation between /y/ and /h/ in non-Greek words, cf. ko-ri-ja-da-na ko-ri- $\left.a_{2}-d a-n a\right),{ }^{426}$ there is no contextual proof that this identification as a TN is correct. Attempts to provide further support from a possible acrophonic origin of FAR are also generally unconvincing. ${ }^{427}$

Likewise, neither of the other two vocabulary words in which *65 is found has a particularly good Greek interpretation. $e-p i-{ }^{*} 65-k o$ and $p e^{*} 65-k a$ are both found in lists of construction materials (PY Vn 46 and 879, respectively), either for building work or for

\footnotetext{
$i$-jo has been interpreted as an o-stem nominative /(h)īyos/ (e.g. Duhoux 2008:356; Jiménez Delgado 2012:58-60) but although on MY Au 102.1 $i$-jo is clearly an appellative, it could equally be the name /Iōn/, while on $\mathrm{KN} \mathrm{V(7)} \mathrm{1523.4a.5a} \mathrm{the} \mathrm{difficulties} \mathrm{in} \mathrm{establishing} \mathrm{the} \mathrm{relationships} \mathrm{between} \mathrm{the} \mathrm{various} \mathrm{people}$ listed mean that its alternative interpretation as the participle /iōn/ may be preferable (Ruijgh 1967:363; Godart 1974:273-5).

${ }^{424} \mathrm{Cf} . \mathrm{KN}$ X 5509 ri- $^{*} 65[\text {, X } 9519]^{*} 65-n o$, Xd 8132 ri- $^{*} 65[\text { [, and Uf(1) } 7488]^{*} 65-n o$.

${ }^{425}$ See EDG:xxxi; Steele-Meißner forthcoming.

${ }^{426}$ Note also that ${ }^{*} 65$ in this term is written over erasure, possibly [[jọ] $\left(K T^{5}\right)$. Meißner 2008:513 suggests that the alternation between ri- $^{*} 65-n o$ and ri-u-no in the RCT tablets could be because the loss of intervocalic*/h/ was still in progress at the time of this deposit.

${ }^{427}$ Duhoux 2008:346-7 suggests that FAR could have originated as an abbreviation from the root */y(e)w-/

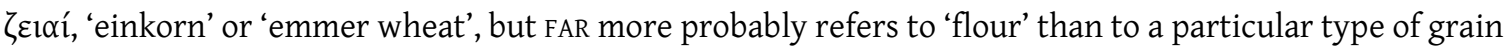
(cf. p.134, n.398). If AB65 was ideographic in LA, then it could well be acrophonic from a non-Greek word;

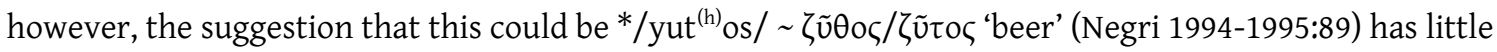
to recommend it. There seems no good reason why LB should adopt an LA ideogram for 'beer' to stand for a cereal product, even if $\zeta \tilde{u} \theta \circ \varsigma / \zeta \tilde{v} \tau o \varsigma$ were not of completely obscure origin (CDE; EDG q.v.).
} 
ship construction. ${ }^{428}$ L.R. Palmer's original suggestions of /epizug(i)on/ 'beam' and /perzuga/ 'joined together' both depend largely on comparisons with rarely-attested

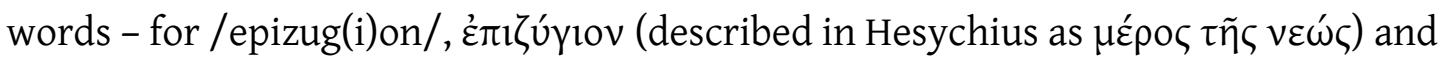

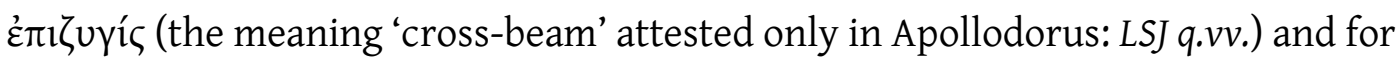
/perzuga/, $\pi \varepsilon \rho i ́ \zeta ৩ \xi$ (likewise attested only in Hesychius with the meaning 'joined together'; more usually 'spare' or 'left over': LSJ q.v.) (29 $^{429}$ and /perzuga/ would also be an unparalleled example of apocope in Mycenaean. Moreover, both of these interpretations require an explanation of why */y-/ should be represented by $j$ - here, but by $z$-in all other occurrences of the root*/y(e)ug-/ in LB. ${ }^{430}$

The suggestion of Duhoux 2008:347 that the change of $* / y-/>/ d z-/$ (whatever stage of this process the $z$-series may have represented $)^{431}$ was slower to operate on forms in */yu-/ than */yeu-/, and had therefore not yet taken place in these forms at the time of the LB tablets, ${ }^{432}$ is without supporting evidence (there are no other forms in */yu-/ attested in Mycenaean) and is made implausible by the facts that examples of */y-/ which are spelt $\zeta$ - in alphabetic Greek are consistently spelt $z$ - in $\mathrm{LB},{ }^{433}$ and that in many cases Mycenaean has progressed further than any later Greek dialect with sound changes

\footnotetext{
${ }^{428}$ Baumbach 1972; Hocker-Palaima 1990-1991.

${ }^{429}$ L.R. Palmer 1955b:43 and 1963b:19-20, 296-7, 367. Originally di-*65-pa-ta, which appears on a textile

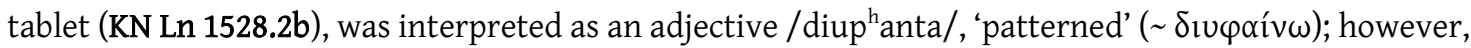
this is now interpreted as a probable WN, for which Palmer's second suggestion of /Diup ${ }^{\mathrm{h}}$ antās/ (an 'occupational PN': L.R. Palmer 1963b:19-20) is evidently not secure.

${ }^{430} \mathrm{Cf}$. ze-u-ke-si (PY Ub 1318.4), ze-u-ke-u-si (PY Fn 50.9) and the abbreviation ZE (KN, PY, and KH, passim).

${ }^{431}$ On the value of the $z$-series, see p.11, n.22; since its actual phonetic value is not of particular importance for this discussion, I conventionally transcribe it as /dz-/ here.

${ }^{432} \mathrm{Cf}$. Chadwick 1983:82's suggestion that this should be seen as evidence for a standard and substandard dialect, with */y-/ retained in the latter.

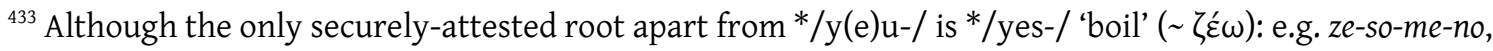
a-re-pa-zo-o/a-re-po-zo-o); for this and other possible examples, see Melena 2014b:50.
} 
involving palatalisation and affrication. ${ }^{434}$ A delayed operation of this particular change in certain, completely ad hoc circumstances therefore does not seem likely.

An alternative suggestion (made by Melena 2014a:78-9) is that the sound change of */yu-/ > /dzu-/ took place at some point between the creation of LB and the time of the attested tablets. The sign originally representing ju therefore came to represent $z u$ in words which had undergone this sound change (and, similarly, when preceded by a vowel $*_{j u}$ could represent /hu/, due to the sound change of $* / y />/ h /$ intervocalically). However, even if we assume that the latter sound change was indeed complete (which is far from certain) ${ }^{435}$ saying that $j$ - could be a historical spelling for $/ \mathrm{h} /<* / \mathrm{y} /$ is not the same thing as saying that $j$-could represent $/ \mathrm{h} /$. Since LB already has a means of marking intervocalic aspiration, namely spellings without $j-,{ }^{436}$ it would be perverse to use a j-series sign in this way; and indeed the $j$-series is never used to write aspirates from sources other than */y/ (e.g. */s/). ${ }^{437}$ Likewise, historical spellings in $j$ - for */y-/ > /dz-/ could in theory have existed if this sound change were fairly recently completed; but again we face the problem that there is no evidence of this in any other form in $* / y-/$, including other derivatives of*/y(e)ug-/. Presumably, therefore, this theory also relies on the delayed operation of $* / \mathrm{yu}^{-} / \mathrm{>} / \mathrm{dzu}-/$, leading to the existence of historical spellings in the extant tablets in this case but no others; quite apart from the severe structural difficulties inherent in assuming that a single sign could represent consonants

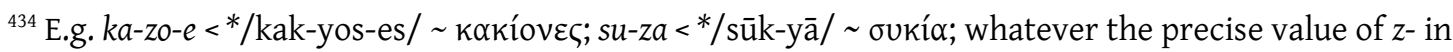
these forms, they have clearly undergone some form of palatalisation which did not occur in later Greek dialects. Palatalisation has progressed even further in the feminine adjectival suffix -we-sa /-we(s)sa/ <

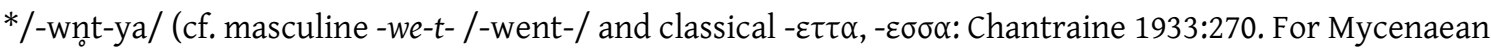
examples, see Bartoněk 2003:236-8).

${ }^{435}$ Cf. p.11, n.20.

${ }^{436}$ Meißner 2008:515.

${ }^{437}$ Thompson forthcoming.
} 
from two (or three) different series, ${ }^{438}$ this is therefore just as ad hoc, and just as unsupported, as the first explanation.

No alternative interpretations have, to my knowledge, been proposed for e-pi-*65-ko;

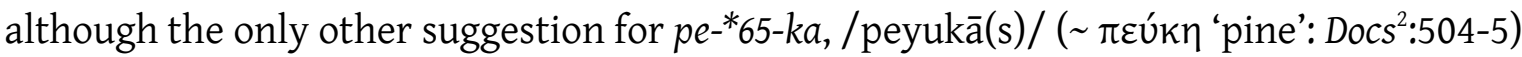
would similarly fit the construction context, it would still require an explanation of the spelling in j-, which, as Docs ${ }^{2}: 571$ points out, would not be expected for the root */peuk-/ (cf. $C D E ; E D G$ q.v.). It may be better to admit that both of these, like the majority of terms on Vn 46 and 879, are simply obscure terms which may have no later Greek equivalent. ${ }^{439}$ Nonetheless, despite the lack of other certain support, the identification of $i{ }^{*} 65$ as /(h)ìyus/, together with the evidence from the sign's distribution and the probability of an inherited sign ju existing, makes *65 probably the best candidate of all the undeciphered signs for a potential decipherment.

\section{*65/FAR at Thebes}

The debate over the Theban examples of this sign concerns whether they should all be identified as $* 65,{ }^{440}$ as FAR, ${ }^{441}$ or as a mixture of the two. ${ }^{442}$ The last option seems in principle the most plausible, since both the syllabographic and ideographic forms are

\footnotetext{
${ }^{438}$ Pace Melena 2014:83's argument that this would be possible due to the small number of occurrences of both $j u$ and $z u$. The parallel interpretation of ${ }^{*} 63$ as $j i / z i$ appears to be based entirely on the prior assumption of the values $j u / z u$ for ${ }^{*} 65$, and therefore cannot offer any support (cf. pp.127-8).

${ }^{439} \mathrm{Cf}$., for instance, the other terms attested alongside these: $a$-ti[ and ko-ni-ti-ja-ja with pe-*65-ka; ta-to-mo, $a-r o-w o$ with $e-p i-{ }^{*} 65-k o$. The only one of these which is at all identifiable is ta-to-mo $=/$ stat $^{\mathrm{h}} \mathrm{mos} /$, 'pillar/post'; it is unclear even what part of speech the others are, so this offers little help with interpreting $p e^{-*} 65-k a$ and $e-p i-{ }^{*} 65-k o$.

${ }^{440}$ Palaima 2006.

${ }^{441}$ TFC, passim; see also Aravantinos et al. 2004:23-6.

${ }^{442}$ Killen 2006:103-6.
} 
found at all other sites where this sign is attested, but it is necessary to examine each individual attestation in detail in order to establish its probable reading, before considering possible interpretations of those examples which appear to be *65 based on its probable value of $j u$. Throughout this section I shall refer to examples of this sign as W until or unless they can be identified as (probably) either *65 or FAR.

W is attested up to 25 times in two series at Thebes, the Fq-series (normally recording distributions of grain) $)^{443}$ and the Gp-series (normally recording distributions of wine).

\section{Probable examples of FAR}

\section{Gp 153.2, 303.1}

The only way of completely certainly identifying an example of FAR would be if $W$ were followed by an unambiguous dry-measure metrogram ( $\mathrm{T}$ ) in the Gp-series, in which case wine could not be the commodity being distributed and another ideogram would be required. ${ }^{444}$ The only certain example of $\mathrm{T}$ attributed to this series, on $G p 153.2$, follows an uncertain (but reasonably probable) attestation of $W^{445},{ }^{45}$ wile $G p 303.1$ has what I regard as a certain example of $W^{4},{ }^{446}$ but followed by a very uncertain $T,{ }^{447}$ and $G p 149.1$ has an

\footnotetext{
${ }^{443}$ Denoted by HORD, on which see p.134, n.398. As the type of grain represented by this ideogram is controversial but not particularly important for this discussion, I shall refer it to it throughout simply as HORD or 'grain'.

${ }^{444}$ Of course, this cannot distinguish between $* 5$ and FAR in the Fq-series, since both HORD and FAR would be measured in dry-measure units. There are no examples in the $\mathrm{Gp}$-series of $W$ directly followed by an unambiguous liquid-measure metrogram (s), so this cannot provide evidence for examples of *65. ${ }^{445}$ TFC read this as uncertain, while Killen 2006:104 accepts it as secure; following autopsy I regard it as probable but would maintain TFC's dotted reading.

${ }^{446} T F C^{1}$ and $T F C^{3}$ read this as uncertain, but $T F C^{4}$ and Killen 2006:104 regard it as certain, as do I following autopsy of this tablet.

${ }^{447}$ The possible example of $\Varangle$ on $\mathbf{3 0 3 . 2}$ is too uncertain to be included in this discussion, as is the one on Gp 313.1.
} 
uncertain T but with no ideograms preserved. It is thus probable that $G p 153$ and 303 record distributions of FAR (and possible that Gp 149 does likewise) ${ }^{448}$ However, the attribution of these tablets to the Gp-series seems questionable, since none of them has an example of viN (and 149 has no ideograms at all), recipients which recur in this series, or a secure attribution to a scribal hand: ${ }^{449}$ hence my classification of all three as $G p .{ }^{450}$ Thus, the probability that these tablets do show examples of FAR cannot necessarily provide support for identifying other Gp-series examples of $\$ as FAR.

\section{Probable examples of $* 65$}

\section{Gp 110.2, 124.1.2}

On Gp 110.2, a word-divider appears between $\$$ and v. With a reading FAR, this would be the only instance in this series of a divider between an ideogram and a metrogram, whereas a divider between a word ending -*65 and a following ideogram or metrogram would be unproblematic. ${ }^{451}$ Although a single parallel for the use of a divider between an ideogram and a metrogram can be found at Knossos, ${ }^{452}$ the reading $]^{*} 65$, v 2 seems preferable. ${ }^{453}$ A similar argument applies to $\mathrm{Gp}$ 124.1, which has a word-divider between

\footnotetext{
${ }^{448}$ Palaima 2006:147 argues that $\$$ on 303.1 should be identified as *65 due to the signs' spacing, as i-je-re-wi-jo and $W$ 'run together'; but if anything, $W$ seems to be closer to the following ț than the preceding sign-sequence.

${ }^{449} 149$ is attributed by TFC to H306?, but this is presumably based on the assumption that it forms part of the Gp-series, which are mostly written by $\mathrm{H} 306$; a tablet whose only certainly-identified sign apart from numerals is $\mathrm{V}$ can hardly be securely ascribed to a hand.

${ }^{450} \mathrm{Cf}$. the statement of Palaima 2006:147 that 153 seems to belong to a different 'set' of tablets.

${ }^{451}$ Palaima 2006:147. The numerous parallels for this at Thebes include an example on 110.1 (]qe, vIN S 1); unattributed examples involving a metrogram are seen on Up 432.4(]$\left.a_{2}-\mathrm{jo}, \mathrm{v} 1\right)$ and .5 (]wa , v 5) and a probable example in $\mathrm{H} 306$ on Gp 122.2 (a-ki-to, ṿ).

${ }^{452}$ KN Fs 21.1 (H139): ka-u-da, HORD , T 1 [ (Aravantinos et al. 2004:23, n.23). There are also examples of dividers separating ideograms from numerals on KN Dv 1191, Dv 1205, and perhaps K 740.3 (Duhoux 1999:228).

${ }^{453}$ This also makes it less difficult to explain why the following entry, mo-ne-we s 1 , records a distribution of wine (as shown by the liquid-measure symbol s) without specifically indicating this with the vin ideogram.
} 
W and a following ideogram: ]ko , VIN V 2[. The argument that $\Varangle$ should be read FAR, and that FAR, VIN could indicate a mixture of the two products, ${ }^{454}$ is extremely difficult; although other examples of sequences of two ideograms exist, ${ }^{455}$ including some separated by a divider, ${ }^{456}$ none provides a good parallel for this specific usage indicating two separate products being mixed together. The reading *65 is therefore strongly to be preferred in this case. ${ }^{457}$

\section{Fq-series}

The frequent appearance of $\Varangle$ on the same tablet as HORD in the Fq-series is not, in itself, necessarily problematic for its interpretation as FAR (pace Palaima 2006:147), any more than its appearance alongside VIN is necessarily problematic in the Gp-series, since at other sites FAR always appears alongside other commodities. ${ }^{458} \mathrm{Fq} 214,229$, and $254[+] 255$,

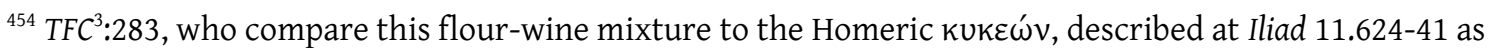
being made of wine and barley-flour.

${ }^{455}$ In the KN Dp-series, ovIS ${ }^{\mathrm{m}} / \mathrm{ovIS}^{\mathrm{f}}$ are juxtaposed with LANA to indicate that the wool in question is from male or female sheep, while on KN D 7252 OVIS VIIR, if correctly read, might indicate the status of the men as shepherds (Killen 2006:103, n.73). AES and AUR may precede an object to denote 'made of bronze/gold', e.g.

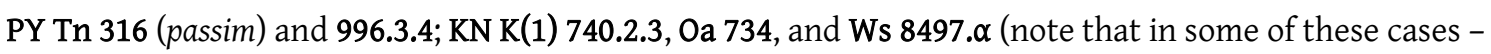
PY Tn 316 v.5.6; KN Oa 734, Ws 8497. $\alpha$ - the two ideograms are actually formed into a monogram). In such entries, the first ideogram is functionally parallel to an adjunct: compare ovIS ${ }^{\mathrm{m}}$ LANA 'wool, specifically from male sheep' and AES * $205^{\mathrm{VAS}}$ 'vase, specifically a bronze one' to ki ovIS 'sheep, specifically young ones'. ${ }^{456}$ In the sequences DA, LANA and DA , ku LANA (TH Of 34,40 ), the divider separates DA (denoting the 'supervisor' status of the person preceding this) from the 'proper' ideographic component of the entry; note the lack of a divider between $k u$ and LANA, where the former functions as a qualification of the latter (TT II:92-3; Killen 1983b:122-3). On KN Ap 639.4 (cited as a parallel by Aravantinos et al. 2004:23, n.23), the exact meaning of *18-to-no , / ț , MUL 2 is unclear (either '*18-to-no and her daughter' or 'the daughters of *18-to-no'), but the tu appears to have been added after muL had been written: the dividers (the only ones on this tablet) were therefore probably added to mark out the tu as an adjunct/abbreviation (note the small size and crowding of the signs here), rather than part of the preceding syllabic sequence (see Killen 1966:210-2).

${ }^{457}$ Cf. Palaima 2006:147.

${ }^{458}$ E.g. the KN Fs-series lists HORD, ME+RI, NI, OLE, and VIN as well as FAR; the PY Un-series records an even larger range of foodstuffs and livestock as well as A+RE+PA, LANA and TELA; and MY Fu 711 lists FAR alongside $\mathrm{CYP}+\mathrm{O}$, HORD, and NI. On the other hand, these examples are all characterised by the large number of 
are, however, the only ones to contain both examples of $\underset{W}{ }$ and a totalling entry at the end, and in none of these cases is there a total given for FAR (nor are there any other examples of FAR appearing in a totalling formula in less complete tablets). All three certainly or probably read HORD after $k u$-su-to-ro- $q a$ ' total'; ${ }^{459}$ none is missing any further lines below, on which a separate FAR entry could have appeared; and although 214 and 229 are not complete at the right, the expected width of the tablet would not leave room for such an entry to follow the HORD total.

If $W$ is FAR on these tablets (and others in the Fq-series), then the quantity of flour distributed to the recipients listed as receiving it must be included in the overall quantity of HORD: certain recipients would receive this grain in milled rather than unmilled form, with FAR acting as a kind of adjunct to the (unwritten) HORD ideogram to specify its milled status (see p.148, n.456 on this qualifying use of ideograms). This is, however, problematic for several reasons. The use of an adjunct to qualify another ideogram which is not in fact written seems difficult (though it is usual in the Fq-series for HORD to appear only in the first entry, and to be understood throughout the subsequent ones), while in general there seems to be a contrast between FAR and HORD/GRA, whether because different types of grain are referred to or, perhaps more plausibly, because milled and unmilled grain are simply categorised separately. As R. Palmer 2008:633 points out, the volume occupied by milled and unmilled grains is significantly different, so that a given volume of HORD-flour would not be remotely comparable to the same volume of HORD. It is therefore extremely hard to justify an

different commodities which recur, in slightly different permutations, on all members of the series, a pattern quite different from that shown by the TH Fq- and Gp-series.

${ }^{459}$ Although Họp with FAR. 
administrative practice (unparalleled in LB) of recording these two substances as a single commodity, unless FAR is in fact an indication that the quantity of HORD specified was to be milled before distribution (but had not yet been at the time of calculating the quantity) - again, a difficult interpretation of an adjunct.

At this point the other Fq-series tablets with the same recipients as 214, 229, and 254 should be brought into consideration, because if these recipients are, exceptionally, receiving milled HORD, this is presumably because they share a similar status, which differs in some respect from the status of the other Fq-series recipients. James 20022003:407-13 distinguishes a hierarchy of recipients on the Fq-tablets: broadly speaking, entries are arranged in a similar order on each tablet, with those receiving larger amounts (v 1 or more) usually appearing in the first half of the tablet and those receiving less (z 1-3) in the second half. This appears to correlate to some extent with other possible indicators of status: for instance, recipients who are also found in other series (who might prima facie be expected to be of higher status to account for their involvement in different areas of activity) are almost all those who appear in the first half of the Fq-series tablets and receive larger quantities of HORD. Examining the recipients who are followed by $\nVdash$ in these tablets within this framework, little can be seen by way of a common pattern: 


\begin{tabular}{|c|c|c|c|}
\hline Recipient & $\begin{array}{l}\text { Frequency } \\
\text { (probable) }\end{array}$ & $\begin{array}{l}\text { Quantities received } \\
(\mathrm{V}=\mathrm{V} 1+; \mathrm{z}=\mathrm{z} 1-3)\end{array}$ & Part of tablet ${ }^{460}$ \\
\hline ra-ke-da-mi-ni-jo & 5 & $\mathrm{~V}$ & Upper; once lower \\
\hline$k a-w i-j o$ & 5,+1 without $\nwarrow$ & $\mathrm{V}$ & Upper \\
\hline o-to-ro-no & 1 & $\mathrm{~V}$ & Upper \\
\hline$a-r a-o$ & 2 & $\mathrm{v} ?$ & Lower; middle? \\
\hline$k u-n o$ & 1 & $?$ & Lower? \\
\hline [.]-tọ-qo & 1 & $\mathrm{v} ?$ & $?$ \\
\hline
\end{tabular}

Although all of these belong to the group receiving larger quantities (where these are known), they have little else in common, and there is nothing apart from the presence of W to distinguish them from others who receive similar quantities. Even on tablets where more than one of these recipients is found, they never all appear directly alongside each other: e.g. Fq 214.7.13, 254[+]255.6.7.13, 258.3.5. There is thus no indication that these individuals form any kind of separate sub-set of the Fq recipients, and so no particular reason to think that they may have been distinguished by receiving milled instead of unmilled HORD. With no satisfactory explanation for the use of FAR in this series, it is therefore far easier in principle to interpret $\$$ as $* 65$ on the majority of the Fq-series tablets (Fq 123.1, 130.3, 214.7.13, 229.4, 254[+]255.6̣.7.13, 258.3.5, 275.3, 284.3, 342.2).

\section{$\underline{\text { Uncertain examples }}$}

\section{Fq 132.1, 236.5}

These two tablets are the exception to this general statement. Not only are there no parallels elsewhere for their recipients ([.]-to-qo, ku-no) being followed by $W_{i} ;^{461}$ but they are not in my opinion even certainly part of the Fq-series, since they lack any examples

\footnotetext{
${ }^{460}$ Based on relatively complete tablets.

${ }^{461} \mathrm{ku}$-no is also attested on Fq 205.3, Gf 163.1, and Gp 150.2, but is not followed by $\Varangle$ in any of these instances.
} 
of HORD and also appear to differ significantly from the usual formatting of this series (particularly in the spacing between the recipients and the following metrograms). While 132 is attributed to $\mathrm{H} 305$ (who was responsible for most of the Fq-series), 236 is attributed to H304, who has not written any certain Fq-series tablets. Palaima 2006:147 argues that $\nVdash$ should be read as $* 65$ on both due to its position close to the preceding syllabograms (while the z signs are positioned further to the right); however, due to the damaged condition of both tablets we cannot say how other ideograms were positioned, nor how much space there was between $\Varangle$ and $\mathrm{z}$ on the lines in question (the vertical trace on the broken edge of $\mathbf{1 3 2 . 1}$ is more likely to be part of $\Varangle$ than $\mathrm{v}$, as read by TFC).

\section{Gp 144.1, 215.1.2.}

The interpretation of $\$ on these two remaining tablets is similarly uncertain due to their incomplete state (it is not certain whether a VIN ideogram may have appeared in a preceding entry), the lack of any other examples of these recipients (]-we, qe-da-do-ro, $a-] k o-r o-d a-m o$ ) followed by $\Varangle$ for comparison, and the positioning of $\nVdash$ roughly equidistant from to the preceding sign-sequences and the following metrograms.

As was stated above to be the most plausible situation in principle, we therefore have probable examples at Thebes of $\$ in both syllabographic and ideographic form. The former are evidently of greater importance for the purposes of this thesis, and will therefore now be discussed in the light of the probability that *65's value is ju.

\footnotetext{
${ }^{462}$ Note that I also regard these tablets' series attribution as uncertain due to the lack of vin ideograms; 215 has no scribal attribution and $\mathbf{1 4 4}$ is tentatively attributed to H305?, who has no certain Gp-series tablets.
} 


\section{Interpretation of $£=* 65$ at Thebes}

The terms in which probable or possible examples of *65 appear are as follows:

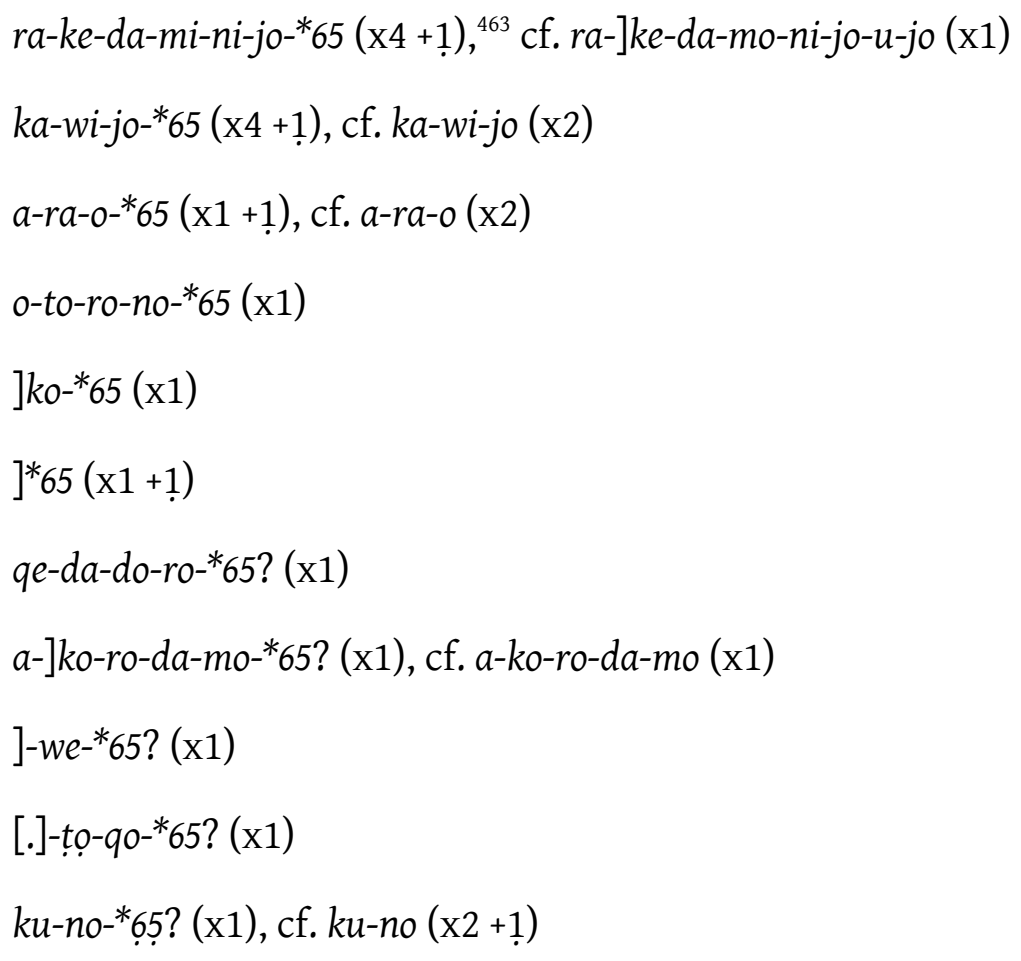

In each case, ${ }^{*} 65$ appears at the end of a (probable) PN or appellative; comparison of ra-ke-da-mi-ni-jo-*65 with the sequence ra-]ke-da-mo-ni-jo-u-jo (Gp 227.2, H306?), in which -u-jo is generally agreed to be the dative /(h)ūiōi/ 'son' (p.141), ${ }^{464}$ suggests that -*65 may have a similar function, and that the individuals listed above are all recorded with

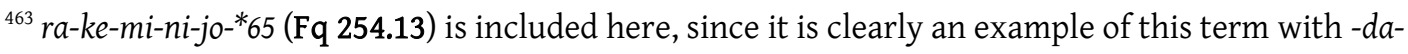
accidentally omitted.

${ }^{464}$ The suggestion of a dual form /Lakedaimoniō (h)üiōo (Witczak 2011) is based entirely on the assumption that this must refer to (a) deity/deities (i.e. the Dioscuri), which is not necessary even if the Fq- and Gpseries are considered to have a generally religious character $\left(T F C^{1}: 214-5\right.$, for instance, consider this to be an individual playing a role in Theban cult practices). Likewise, Witczak's interpretation of -jo-* 65 as a genitive/dative dual ending /-oyun/ depends on similar assumptions concerning the Dioscuri (the presence of multiple other pairs of individuals is not adequately explained) as well as the existence in Mycenaean of an oblique dual ending corresponding to Arcadian -olvv (cf. the more usual -o $(1)$ lv: Buck 1955:89) - not impossible, but unproven (there are no certain LB examples of the $a$ - or o-stem gen./dat. dual; Bartoněk 2003:165-6, 188 gives -o-i /o(y(y))in/? for both, but this seems to be based only on the interpretation of wa-na-so-i as a possible dual, whereas it is generally interpreted as a dat./loc. plural).
} 
reference to their fathers (Palaima 2006:146). ${ }^{465}$ Since there is no plausible way to interpret the majority of the terms preceding $-u-j o$ and $-{ }^{*} 65$ as patronymic adjectives, ${ }^{466}$ nor as fathers' names in the genitive, ${ }^{467}$ the best explanation seems to be that these are the sons' names (in the dative, or nominative of rubric) ${ }^{468}$ in apposition to - $u-j o /-{ }^{*} 65$, so that, e.g., ra-ke-da-mo-ni-jo/ra-ke-da-mi-ni-jo plus -u-jo/-*65 means 'Lakedaim(o)nios, the son' or 'L. Jr.'. Examples of the same names attested without -*65 could therefore be either these individuals' fathers, or the sons with the specification of 'Jr.' omitted. ${ }^{469}$

\footnotetext{
${ }^{465}$ The assumption that ra-ke-da-mi-ni-jo /Lakedaimnios/ and ra-ke-da-mo-ni-jo /Lakedaimonios/ are both forms of the same name and refer to the same person seems a reasonable one; although these may be different morphological formations, such variation in PNs is not unknown in alphabetic Greek (Duhoux 2008:359-60). Alternatively, since ra-ke-da-mo-ni-jo is attested only once, this could be an error or a variant spelling by analogy with /Lakedaimōn/ (note also that this is used by H306?, while H305 writes ra-ke-da-mini-jo).

${ }^{466}$ Only ra-ke-da-mi-ni-jo/ra-ke-da-mo-ni-jo and ka-wi-jo could formally be patronymics; the former is (at least in origin) an ethnic adjective while the latter is also attested alone, implying that it is a PN (Killen 2006:105, n.77). There are no other examples of a person being referred to by a patronymic alone in LB (Bartoněk 2003:422-3).

${ }^{467} \mathrm{ra}$-]ke-da-mo-ni-jo-u-jo could easily be haplography for $\mathrm{ra}$-]ke-da-mo-ni-jo-<jo->u-jo /Lakedaimonioyo (h) ūioīi/ (TFC'1:298), but clearly a systematic haplography of this type cannot be assumed for all these terms. Some of these could in principle be genitives (e.g. o-to-ro-no, a-ra-o, and ku-no, which TFC $C^{1}$ :196-7 interprets as /kunos/ or /kunōn/), but as the last two are also attested without - 65 this seems unlikely; these and all the other terms in question are most probably either o-stem datives in /-ōi/ or nominatives of rubric in /-os/. The suggestion that they are o-stem genitives in /-ō/ (Duhoux 2008:358-9) is implausible; Thompson forthcoming [2017] has convincingly shown that supposed examples of this /-ō/ genitive are most likely to be due to scribal errors (on this issue, see also Chadwick 1958; Pisani 1959; Morpurgo Davies 1960; Lejeune 1972c:17-18; Hajnal 1995:247ff.; Bernabé-Luján 2006:144-6; Jiménez Delgado 2013). Admittedly it remains difficult to explain the entry ]a-me-ro, qe-da-do-ro- 65 (Gp 215.1), with a sequence of two PNs of which the second is followed by 'son', but the break at the beginning of this entry means that further information about the relationship between these two PNs may have been lost; qe-da-do-ro is therefore not necessarily the son of $a$-me-ro even if $W$ here $={ }^{*} 65$ and not FAR. Compare also the entry re-wa-ko a-me[-ro on Fq 130.3, in which two recipients who each appear alone elsewhere appear to be in asyndeton with each other in a single entry $\left(T F C^{1}: 297\right)$.

${ }^{468}$ On the case of these terms, see further p.156.

${ }^{469}$ Palaima 2006:146. Cf. pa-se-ri-jo ko-wo (MY Oe 121.2: Duhoux 2007:101) and u-wa-si-jo ko-wo (KN Ai 115: Thompson forthcoming [2017]), which could be 'P. Jr.' and 'U. Jr.'; however, these could also be interpreted as patronymics (Duhoux 2008:285). The other possible instance of this usage, on KN V 1523, is highly problematic (see p.141, n.423). There is thus no certain parallel for this, but it remains a perfectly plausible one (the alternative suggestion by Jiménez Delgado 2012:59 that parenthood could be denoted by the father's name in the dative preceding 'son' is much less plausible, since as Jiménez Delgado points out, the dative of possession is generally restricted in classical Greek to use with pronouns).
} 
How is $-^{*} 65=$ 'son' to be phonetically interpreted? If ${ }^{6} 65$ is $j u$, then this cannot represent the form of the word 'son' already attested at Thebes, /(h)ūio-/, either fully or (as suggested by J.L. Melena and accepted by Palaima 2006:148; see also Melena 2014a:78) in abbreviated form: the latter relies on the assumption that ju could represent / hu/ even when this originated from */s/ rather than */y/, which has already been shown to be unfounded (pp.144-5). Nor can it represent the other known Mycenaean word for 'son' in the same form in which it is represented at Pylos, $i^{-*} 65$ /(h)iyus/, unless an explanation can be provided as to why the $i$ - is not written; but while the suggestion that this is due to haplology could apply to sequences such as ra-ke-da-mi-ni-jo-*65, if it is assumed that the aspiration was lost and the words run together in speech as /Lakedaimniōi-īyu-/ > /Lakedaimniōìuu-/ (Palaima 2006:148), it is more difficult to assume that such haplology would be consistent even in speech, let alone consistently represented in writing.

It seems possible in principle that -*65 could represent another form of the word 'son' derived from either */sūyus/ or, more likely, from $/\left(\right.$ h)ìyus $/ ;^{470}$ in practice, the only possible form whose representation by ${ }^{*} 65=j u$ seems orthographically plausible is /(h)yus/. ${ }^{471}$ Although this interpretation involves assuming that two different forms of the word 'son' could have existed at the same site at the same time, this seems acceptable given the existence of multiple forms of this word throughout the history of Greek, as well as the fact that at least two different outcomes of*/sūyus/ are already attested in Mycenaean, albeit at different sites. It also requires identification of the case

\footnotetext{
${ }^{470}$ The suggestion that the first syllable of */sūyus/ > */(h)ūyus/ could have been lost by dissimilation (Duhoux 2007:100 and Duhoux 2008:356) does not seem particularly plausible. Duhoux's only suggested Mycenaean parallel is a-pi-po-re-we /amphiphorēwes/ a-po-re-we /amphorēwe/, cf. alphabetic $\dot{\alpha} \mu \varphi і \varphi о \rho \varepsilon u ́ \varsigma \sim ~ \alpha ̉ \mu \varphi о р \varepsilon u ́ c$, but the loss of the first syllable of a disyllabic word seems much less likely than that of a medial syllable from a polysyllabic word.

${ }^{471}$ See Thompson forthcoming [2017], who interprets $-{ }^{*} 65$ as /hyus/.
} 
of $-{ }^{*} 65=/(\mathrm{h}) \mathrm{yu}-/$ : the expected dative case is ruled out by the fact that a form in - we would be expected, either-e-we /-ewei/ (cf. i-je-we, p.141) or -u-we /-uwei/ (the regular Mycenaean $u$-stem dative, e.g. e-ri-nu-we, KN V 52; ko-ru-we, TH passim. ${ }^{472}$ Although e-ri-nu, found in a list of recipients on $\mathrm{KN} \mathrm{Fp}(1) 1.8$, is frequently quoted as a possible example of a $u$-stem dative in /-ui/, ${ }^{473}$ it is better seen as a nominative of rubric). ${ }^{474}{ }_{-}^{*} 65$ would therefore have to represent the nominative of rubric /(h)yus/; but again, this seems acceptable given the frequency of these forms in the LB records. Whether all the preceding terms should equally be seen as nominatives of rubric, or whether this formulaic use of 'son/Jr.' encouraged its appearance in the nominative regardless of the preceding case, is an open question.

However, this interpretation is not without problems, since the process by which a form /(h)yus/ would have arisen from /(h)iyus/ is unclear. R. Thompson (pers. comm.) suggests that this could have been produced by a process of yodization similar to that seen in the cases of $\mathrm{ra}_{2}$ and $t a_{2}(/ \mathrm{iV} />/ \mathrm{yV} /:$ pp.49, 53); but this involves assuming not only that the original */y/ of */(h)iyus/ had already become /h/, but also that this resulting / $\mathrm{h} /$ had itself been lost, producing a sub-phonemic glide between the $/ \overline{1} /$ and $/ \mathrm{u} /$, so that $i^{*} 65$ would be $/(\mathrm{h}) \bar{i}^{\mathrm{y}} \mathrm{us} /$ rather than $/(\mathrm{h}) \overline{\mathrm{i}} \mathrm{yus} /$. The spelling alternations between $-j$ - and $-\varnothing$ - which form the basis of the usual assumption that the $* / y />/ h /$ change is either in progress or recently complete (on which see p.11, n.20 and p.144) do not appear to support the idea that this change had progressed far enough to result in

\footnotetext{
${ }^{472}$ Further examples in Bartoněk 2003:275-9.

${ }^{473}$ E.g. Bartoněk 2003:278; Hajnal 1995:32; cf. Palaima 2006's assumption that -*65 would represent /-yu(i)/ if not an abbreviation.

${ }^{474}$ Killen 2006:105. In any case, /Erinūs/ is a ū-stem and /(h)īyus/ a ŭ-stem: compare classical Attic $\sigma \tilde{u} \varsigma$, dat. бuí, and $\pi \tilde{\eta} \chi \breve{v} \varsigma$, dat. $\pi \dot{\eta} \chi \varepsilon \varepsilon$. Although various forms of the dative singular of 'son' are attested in classical

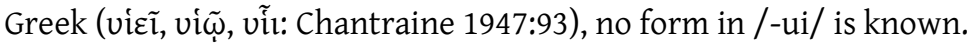


the loss of the /h/, though of course the lack of specific representation of $/ \mathrm{h} /$ in most cases makes it difficult to be certain. The alternative possibility, that /(h)iyus/ > /(h)yus/ by means of some sort of assimilation of the /i/ to the /y/ (Duhoux 2007:100), seems equally problematic due to the length of the vowel. Ultimately, it remains highly probable that some examples of $\mathcal{W}$ at Thebes should be read as $-* 65$ rather than FAR, and that these do represent a form of the word 'son'; but unfortunately, in the current state of the evidence, none of the suggested interpretations of this form is completely satisfactory.

\section{$\underline{2.11 * 79}$,}

${ }^{*} 79$ is one of the rarer signs, with 6-7 examples from Knossos, Pylos, and Mycenae. ${ }^{475}$ Its basic form is a roughly oval shape (KN H103, MY H55, Cii hand of PY Va 404?); this may also have a stroke in the middle and/or a series of small strokes around the outside (KN H117 has both; PY H1, H41, and H42 only have the outer strokes).

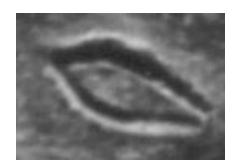

KN Ap $618.2(\mathrm{H} 103)$
Imageremoved for copyright reasons

KN Dv 1501 (H117)

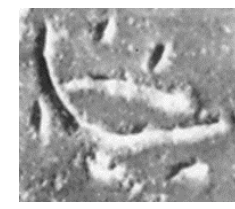

PY Va $482(\mathrm{H} 42)^{476}$

Thus, the simpler and more elaborate forms are both seen at Knossos and on the mainland, and probably at all sites with more than one example of this sign. ${ }^{477}$

\footnotetext{
${ }^{475}$ A previously-identified example from the RCT, wo-*79 (KN V(1) 7049, H124-G: Killen-Olivier 1968:136), is excluded here, since I follow KT $T^{5}$ and COMIK in reading wo-dọ (see also Driessen 1988:140, n.65).

${ }^{476}$ Photos: MER, except 1501: author (HAM).

${ }^{477}$ Although all certain examples of *79 at Pylos are of the more elaborate form, PY Va 404 is a plausible example of the simpler form.
} 
*79's LA antecedent, AB79 泎 (of which there are up to 34 known examples) ${ }^{478}$ almost always has the outer strokes and often a stroke or dot in the centre as well; there are no certain examples of $\mathrm{AB} 79$ corresponding to the simpler LB form. ${ }^{479}$ Compare also $\mathrm{CH} 005$ , whose simplified form is essentially identical to the more elaborate form of *79; in fact, the LB forms are in some ways closer to $\mathrm{CH} 005$ than to AB79, since in LA the oval is always upright while in CH and LB it is always (close to) horizontal.

\section{Prospects of decipherment}

${ }^{*} 79$ is attested only in two WNs $\left(a^{*}{ }^{*} 79, \mathrm{ma}^{*} 79\right)$, one possible MN (di-*79-nu[) and one probable noun (e-wi-su-*79-ko). Although the sample size is very small, its distribution (always word-medial or word-final) makes it unlikely to be a vocalic sign; although AB79 is more common word-initially (c.one-third of attestations), this is still far less than would be expected for a vocalic sign. Any of the other values argued in Chapter 1 to be the most likely for a sign inherited from LA are, however, possible, including zi or $z u$ and values in $p_{2^{-}}, n w^{-}, r y^{-}$, or ty-.

It is tempting to use the fact that *79 appears word-finally in two WNs as evidence for a value in $-a$, not because of its appearance in this position per $s e^{480}$ but because $a^{-}{ }^{*} 79$ is likely to represent both the nominative and the dative forms: ${ }^{481}$ on MY Oe 123 , she is the

\footnotetext{
${ }^{478}$ Up to 30 examples in GORILA; also on KN Zb 52 (Macdonald 2000:64, fig.39); SKO Zc 1 (Perna et al. 2005); SY Zb 7 (Muhly-Olivier 2008); and possibly THE 9 (Boulotis 2008, fig. 3), on which see n.479 below.

${ }^{479}$ The possible exception is THE 9: Boulotis 2008: fig. 3 shows a sign with a circular form, resembling the simple form of *79, but which could also be identified as A309; no transcription is given in the publication. ${ }^{480}$ Although $-a$ is the most common ending for WNs in the LB corpus, examples ending in all four other vowels are also known (Docs: 201$)$; the fact of these word-final appearances in WNs has previously been used to argue for values in $-i$ (L.R. Palmer 1955b:43) and - $u$ (Chadwick 1956:238).

${ }^{481} \mathrm{ma}^{*}{ }^{*} 79$ is nominative in both its occurrences at Pylos, referring to a te-o-jo do-e-ra holding land.
} 
recipient of an allocation of wool, while on KN Ap 618 she appears to be one of a group of women workers. ${ }^{482}$ Only $a$ - and $o$-stems have graphically identical nominatives and datives in LB, and the former is far more likely for a WN. ${ }^{483}$ However, in addition to the possibility of a nominative of rubric on MY Oe 123, it is questionable how far a name of non-Greek origin (which $a^{*} 79$ quite probably is) ${ }^{484}$ would have been adapted to Greek morphology. Note also that a value in -a would not match any of those deemed most probable for an inherited sign, since $n w a, r a_{2}$ and $t a_{2}$ are already known and $p a_{3}$ plausibly identified in *56; though of course, further values not currently represented in LB cannot be ruled out.

In principle, the term $e$-wi-su-*79-ko offers the best prospects of deciphering ${ }^{*} 79$, but the meaning of this term is unclear.

a qe-qi-no-me-no

PY Va 482 ]e-re-pa, a-no-po, a-ko-so-ta , ZE, e-wi-su-*79-ko 4 ro-i-ko 2

This tablet appears to be recording a delivery of ivory (e-re-pa) to a worker ( $a$-no-po) from the administrator $a-k o-s o-t a$ of various pairs of ivory tusks $(Z E)$ for use in producing four e-wi-su-*79-ko and two ro-i-ko, either or both of which are to be qe-qi-no-me-no (decorated

\footnotetext{
${ }^{482}$ On this tablet, see Jiménez Delgado 2012:60-1.

${ }^{483}$ It is not impossible that Mycenaean, unlike classical Greek, could have had WNs in /-os/, but there is no positive evidence for this (Docs':405).

${ }^{484}$ There is no evidence either way for the origin of $a^{-*} 79$, but note that $m a^{-*} 79$ may also appear in LA as AB80-AB79. (HT 102.3-4), plausibly a PN from context (Younger (LA Lexicon)).
} 
in some way), ${ }^{485} e$-wi-su-*79-ko is therefore presumably a nominative plural noun (as also implied by the probable example on PY Va 404, which reads only e-wi-su-*79-ko 45). ${ }^{486}$

Although the contexts of Va 404 and $\mathbf{4 8 2}$ do not allow an identification of either $e$-wi-su-*79-ko or ro-i-ko, a possible alternate spelling of the former is found on KN Se 965 and 1007: $e$-wi-su-zo-ko, which refers to an attribute of a chariot. The fragmentary nature of both these tablets means that the precise meaning of this term is likewise impossible to identify from context, but a decorative element made of ivory is entirely plausible. ${ }^{487}$ If $e$-wi-su-*79-ko and $e$-wi-su-zo-ko are in fact variant spellings of the same term, then ${ }^{*} 79$ would have to be either $\mathrm{zO}_{2}{ }^{488}$ which is unlikely since there is no satisfactory explanation of what such a doublet of zo would represent, or $z u$, with an o/ $u$ alternation. ${ }^{489}$ The usual interpretation according to this hypothesis, however, is that this is a compound in /-zugos/ ${ }^{490}$ which leaves this o/u alternation (characteristic of non-Greek words) ${ }^{491}$ completely inexplicable. With no secure evidence as to the actual meaning of either of

\footnotetext{
${ }^{485}$ Killen 2003 (for a range of other interpretations see, e.g., Docs²:348; Lejeune 1958e:213; L.R. Palmer 1963b:368-9; Sacconi 1973). Although Killen's argument is partly based on a comparison with e-wi-su-zo-ko (see below) in my opinion it can stand even without this assumption. The alternative interpretation of $e$-wi-su-*79-ko as a decorative motif, governed by qe-qi-no-me-no, does not seem particularly compatible with either the layout of this tablet or PY Va 404 (cf. Killen 2003:71).

${ }^{486}$ Although a dative would in principle be expected in a record of material to be used 'for making $e$-wi-su- ${ }^{*} 79-k o^{\prime}$, there are parallels for the use of a nominative in this kind of context, e.g. PY Ub 1318.2 o-pide-so-mo ka-tu-ro $o_{2}$, di-pte-ra 4 'bindings for pack-saddles(?): skins: four' (Killen 2003:73).

${ }^{487}$ Chariots in this series are listed, for instance, as e-re-pa-te-jo-pi o-mo-pi 'with ivory [ornamental elements?]' (Se 891.A; cf. e-re-pa-te-o o-mọ, Se 1007.2).

${ }^{488}$ Suggested in Ventris-Chadwick 1953:86, but amended to zu? (see n.489 below).

${ }^{489}$ First proposed in Chadwick-Ventris 1953:202, and widely quoted subsequently. The suggestion of $z i$ (L.R. Palmer 1955b:43) is based on a possible alternation of di-ki-nu-wo (KN Dx 1502) and di-*79-nu[ (assuming a palatal value for the $z$-series: see p.11, n.22), which is a far less likely alternation than e-wi-su-*79-ko e-wi-su-zo-ko.

${ }^{490}$ Docs $^{2}: 348$; Lejeune 1958e:214.

${ }^{491}$ Cf. p.142.
} 
these terms, ${ }^{492}$ nor certain proof that they are in fact the same word, ${ }^{493}$ it is better to view $e$-wi-su-*79-ko and e-wi-su-zo-ko as compounds probably sharing the same first element (whatever the interpretation of $e$-wi-su-) but potentially with different second elements. $z u$ remains a possible value for $* 79$ but (since this would rely on seeing -zu-ko/-zo-ko as a non-Greek element) a currently unprovable one. ${ }^{494}$ Likewise, the lack of contextual evidence means that any attempt to identify the etymology of $e$-wi-su-*79-ko is equally insecure ${ }^{495}$ and the question of this sign's value remains for the moment an open one.

\footnotetext{
${ }^{492}$ Even the interpretation of the first part $e$-wi-su-is problematic. This is usually interpreted as /ewisu-/ < */ewiswo-/ Ėiooc 'equal, evenly-balanced'; however, as pointed out to me by T. Meißner, this relies on a comparison to an artificial form only found in epic (LSJ q.v.) rather than the regular classical toos, as well as the assumption that an athematic suffix /-u-/ could alternate with thematic/-wo-/ (Lejeune 1972f:175, n.1; the only alphabetic parallel given for this is a Messenian TN). A form */wiswo-/, corresponding to loos, may also already be attested in the adjective wi-so-wo-pa-na, though this is an uncertain reading and the second element is obscure.

${ }^{493}$ Interpretations of $e$-wi-su-zo-ko tend to assume it to be an adjective describing a chariot/part of a chariot, e.g. /ewisuzugos/ 'evenly-matched' (Docs':348), /ewisuzorkos/ 'with a matched pair of deer [as

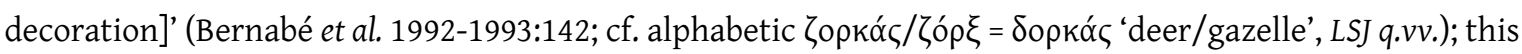
is not compatible with $e$-wi-su- ${ }^{*} 79-k o$ 's probable status as a noun, though as stated above the tablets on which $e$-wi-su-zo-ko is attested are very fragmentary so its adjectival status is far from certain. ${ }^{494}$ The LA distribution of AB79 is very unlike that of the other LA signs corresponding to the LB $z$-series (T. Meißner, pers. comm.): there are no more than two examples of initial AB17 $z a$ in GORILA (ZA 9.1; ARKH 4b.5), and while E. Hallager et al. 1991:37 read ] AB1̣? [ on KH 94.3, there is in fact more space after this sign than before it so it is probably not word-initial. $\mathrm{AB} 20 \sim z 0$ and $\mathrm{AB} 74 \sim z e$ are attested only as isolated signs or in ligatures (AB17 also appears in this format); these could of course be abbreviations or acrophonic ideograms, but need not be. This may make a $z$-value for ${ }^{*} 79$ even less likely.

${ }^{495}$ The suggestion that ${ }^{*} 79=w_{0}$, giving $e-w i-s u-{ }^{*} 79-k o=/$ ewisuworgos $/$ and di-*79-nu[ = /Diwonu[sios $] /$ (Lejeune 1958e:217), gives no precise phonetic value for this proposed doublet. Its interpretation as wyo (Melena 1978 and forthcoming:42-3; Ruipérez 1983) is not easily compatible with either of these interpretations, and the hypothesis that $e$-wi-su-* $79-k o$ and $e$-wi-su-zo-ko represent different outcomes of */ewisu-yoko-/ involves the completely unparalleled and highly implausible scenario of */y-/ producing both /dz-/ and /h-/ in the same root (for a summary of the ongoing debate concerning the reasons for the Greek dual reflex of*/y/, see Meier-Brügger 2003:85-6). Moreover, although wyo has been argued to be a possible value (pp.60-1), this was for a sign invented in LB rather than inherited from LA.
} 


\section{$\underline{2.12 * 82}\}_{2}^{2}$}

*82 has 27-9 certain/possible examples from Knossos, Pylos, and Mycenae, and on an ISJ. It has two main forms, distinguished by the shape of the central part of the sign: Type 1 has a wavy line or ' 3 ' shape, and Type 2 a simple curved line. Type 1 is seen at all sites where *82 is found (Knossos H103; Pylos H1, H2, H6, H25, and the Ci hand of Jo 438; Mycenae H53 and probably H61), ${ }^{496}$ while Type 2 appears only at Pylos (H21, H44, the Cii hand of Vn 19, and S1272 Ciii) and on the ISJ TH Z 853 (probably of West Cretan origin). ${ }^{497}$

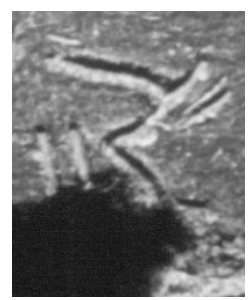

Type 1: KN As(1) 602.4 (H103) PY Un 6.3 (H6)
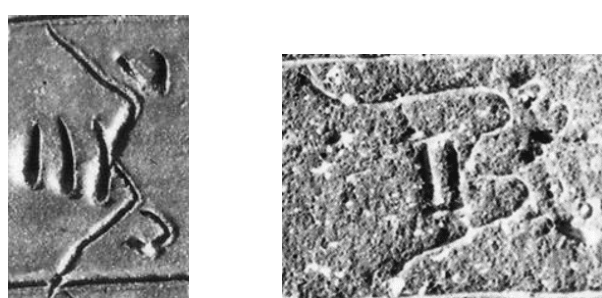

PY Vn $20.3(\mathrm{H} 25)$
Image removed for copyright reasons

MY Fo $101.2(\mathrm{H} 53)^{498}$

As can be seen from the images, this first type shows considerable variation in the precise form of the wavy line or '3' (the more open form seen in e.g. KN H103 or PY H6 is the usual one), as well as in the extra elements present on either side; at the left there may be two verticals (KN H103) or three (usual at Pylos), a rectangular shape (PY H25), or nothing at all (MY H53); the one or two strokes at the top and bottom on the right may be either straight and roughly perpendicular to the main part of the sign (KN H103) or curved and roughly parallel to it (Pylos, Mycenae).

\footnotetext{
${ }^{496}$ The H53 example, on Fo 101.2, was originally identified as *88, but shown to be ${ }^{*} 82$ over erasure by Olivier 1969a:47 (*88 has been formally deleted from the syllabary: Ruipérez 1972b:xvii). The traces on MY V 659.11 (H61) are compatible with a similar form of *82 to that on Fo 101.2. ${ }^{497}$ TSJ:94.

${ }^{498} \mathrm{KN}$ and PY photos: MER; MY photo: author (NAM). The vertical ridges in this last image are part of an erased sign rather than 82 (cf. n.496 above).
} 


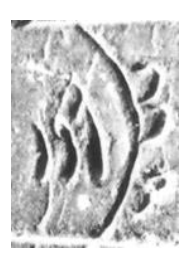

Type 2: PY Vn 19.1 (Cii)

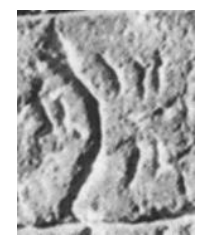

PY Tn 316.5 (H44)

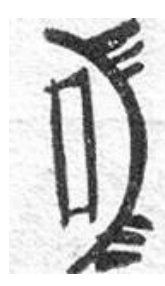

TH Z $853^{499}$

This type shows a similar variation to the first, with two or three verticals or a rectangle to the left, and two or three straight or curved strokes to the right (note also the difference between the more usual small curves next to each other, as seen on Vn 19.1, and H44's three curves in a row). The form of these additional elements is therefore clearly independent of the sign's basic structure. The difference between the straight or curved strokes to the right appears to be a geographical one, with the former found on Crete and the latter on the mainland; on the other hand, the variant with a rectangle to the left, although uncommon, is found both at Pylos and on a Cretan ISJ.

The variation between the two main types of ${ }^{*} 82$ is exactly parallel to that seen in $\mathrm{ku}$, which likewise has forms with a central ' 3 ' shape and others with a simple curve c)?, both of which co-exist at Knossos and Pylos. There is, however, no correlation between the use of particular forms of $k u$ and of ${ }^{*} 82$ (e.g. at Pylos almost all the scribes who use the ' 3 ' shaped * 82 have examples of $k u$ in the simplified form). ${ }^{500}$ In the case of * 82 , although the distinction between these two main types is generally a valid one, they in fact represent two ends of a spectrum: intermediate forms are seen in KN H117 and once in PY H2 (who otherwise uses the more elaborate form), suggesting that the type with a

\footnotetext{
${ }^{499}$ PY photos: MER; ISJ drawing: CIV.

${ }^{500}$ Bennett 1966:303 regards the simplified form of $k u$ as a Pylian innovation to distinguish this sign from *82; however, the simplified form is also seen not only in Knossos but also in LA.
} 
curved central element represents a simplification of an original form similar to the more elaborate first type.

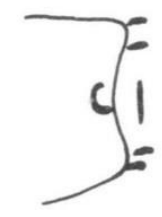

KN Dc 1154.B (H117) PY Ma 225.1 (H2)

This suggestion is supported by the fact that the only certain example of AB82 has a form fairly similar to KN H103's $82:^{502}$

\section{Imageremoved \\ for copyright \\ reasons}

\section{ZA $4 a .7^{503}$}

Since AB81 $(3 \ni)=$ LB $k$ u already shows the variation between a ' 3 ' shape and a curve, it seems probable that AB82 would do likewise were more examples to be attested. It is not clear whether the straight strokes to the right, shared by AB82 and *82 at Knossos, are the original form (with the curved strokes being a mainland innovation) or whether both may have existed within LA.

\footnotetext{
${ }^{501} \mathrm{KN}$ drawing: COMIK; PY photo: MER.

${ }^{502}$ The other two possible examples of AB82, on KH Wa 1003 and 1004, are very uncertain and are therefore excluded here.

${ }^{503}$ Image: GORILA.
} 


\section{Prospects of decipherment}

*82 is attested in all positions in PNs, the TN pi-*82, and the theonym pe-re-*82 (as well as the related term $p e-r e^{-*} 82-j o$ indicating this deity's sanctuary), ${ }^{504}$ it does not appear in any identifiable vocabulary words. Its low frequency of word-initial attestations (only in the $\left.M N{ }^{*} 82-d e\right)$ suggests a $C(C) V$ value rather than a vocalic one; as for *79, any of the most likely values for a sign inherited from LA $\left(z i, z u, p_{2^{-}}, n w^{-}, r y^{-}, t y-\right)$ may be possible. The existence of an initial example may make a palatalised value or one in $n w$ - less likely possibilities, but does not exclude them..$^{505}$

It is frequently argued that $-a$ is the most likely vocalism for this sign: the TN pi-* 82 (presumably dative-locative and/or nominative of rubric) ${ }^{506}$ also appears as the allative $p i-{ }^{*} 82-\mathrm{de}$. The fact that the same spelling can therefore represent the accusative form as well as the dative-locative and/or nominative would suggest either an $a$-stem or an ostem, the former being the more likely given *82's word-final appearance in the female theonym pe-re-*82 and the WN $a-n a-{ }^{*} 82$ (note also that *82 appears in several MNs, but never word-finally). ${ }^{507}$ The attestation of the allative $p i^{*} 82$-de makes the equivalence in spelling of at least two different cases certain, and therefore makes this argument somewhat stronger than it was in the case of ${ }^{*} 79$ (pp.158-9), which only has a probable

\footnotetext{
${ }^{504}$ This deity is shown to be a goddess by her association with women on PY Tn 316. Most attempts at deciphering * 82 rely on etymological interpretations of this theonym and/or comparisons of it to classical goddesses; both are clearly insecure forms of evidence and are excluded from this discussion.

${ }^{505}$ L.R. Palmer 1955a:2, 1956:132, and 1963b:20-1 argues that ${ }^{*} 82$ is mainly found after front vowels and is therefore likely to represent $j$ - (see also p.166, n.508). However, ${ }^{*} 82$ in fact appears after a front vowel in only $56 \%$ of the terms in which it is attested, and after $-i$ in only $33 \%$; although this does not exclude a glide, a higher proportion might be expected for a sign in $j$-. In any case, since ${ }^{*} 65$ is very probably $j u$, there is no plausible value in $j$ - available.

${ }^{506}$ Lejeune 1958e:207. Lejeune argues that this term is also genitive (on PY An 830.12), but a dative-locative seems more likely.

${ }^{507}$ E.g. L.R. Palmer 1955a:2; Lejeune 1958e:207.
} 
dative/nominative equivalence. However, the same objections still apply: although $-a$ is the most frequent WN ending in Mycenaean, all the other vowels are also found; nonGreek PNs or TNs (as pe-re-* $82, a-n a-{ }^{*} 82$, and $p i-{ }^{*} 82$ could all be) may not necessarily be made to conform to Greek morphology; and if the assignment of an a-vocalism is correct, this rules out all of the values judged most probable in principle for an inherited sign. Nonetheless, the probability that 82 has a vocalic value of - $a$ seems a reasonable, though not a certain, one.

Among the many different values which have been proposed for $* 82{ }^{508}$ the only ones which seem at all plausible in principle are $w y a,{ }^{509}$ sya $^{510}$ and $s w a .^{511}$ All of these were argued in Chapter 1 to be possibilities, since these (secondary) palatalised values might be used particularly in adjectival formations in /-ia/ (pp.60-1), while swa might be more sporadically useful, primarily in representing PNs and TNs containing this sequence (p.47); although these were regarded as values which might more plausibly have been invented within LB, the inheritance of further palatalised or labialised values from LA remains a possibility. As remarked above, however, the word-initial appearance of ${ }^{*} 82$ in *82-de makes the palatalised values less likely (and neither has any particularly good

\footnotetext{
${ }^{508}$ E.g.ja $a_{2}$ (L.R. Palmer 1955a:2, 1956:132, and 1963b:20-1; Lejeune 1958e:206-10; Probonas 1983-1984), jo (Doria 1958), $\mathrm{ka}_{2}$ (Lurja 1955:9, 17), $\mathrm{ku}_{2}$ (Furumark 1954 Part I:112, Part 2:53; Landau 1958:14), kuta (Sittig 1954:68), kwa (Gallavotti 1958b:76 [370], n.1; de Venuto 1968), qu (Georgiev 1955:7 and 1956:59-60). ${ }^{509}$ Gallavotti 1956a:25, 1956c:226, and 1957a; Witczak 1993b:120-1; cf. Doria 1972:46-51 (wa $)$. ${ }^{510}$ Pugliese Carratelli 1957:4-6; Survinu 1968; cf. sa ${ }_{2}$ (Lejeune 1958e:210; Ruipérez 1958; Olivier 1969a:47). ${ }^{511}$ Gallavotti 1959:164-5; Chadwick 1968a:64-5; Sergent 1977; Melena 1983:263-6 and forthcoming:45; Quattordio-Moreschini 1987.
} 
evidence to support it), ${ }^{512}$ so that swa might be the best of these options. ${ }^{513}$ This value, however, is similarly lacking in any firm support: it is based primarily on the

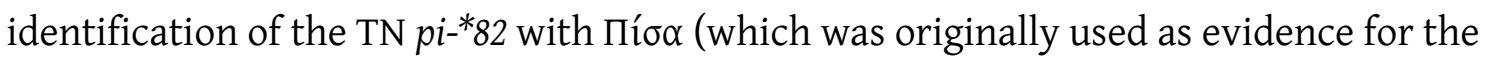
value $s a_{2} /$ sya: p.166, n.510), the reconstruction of */Piswā/ being based entirely on a comparison with pi-sa-wa-ta (an MN on KN B 1055.2), interpreted as /Piswātās/. ${ }^{14}$ Since the latter is found only at Knossos, a link to a mainland site - whether this is identified as the classical Pisa in Elis (Sergent 1977) or a homonymous location in Messenia (Chadwick 1968a:64-5) - does not seem particularly likely. ${ }^{515}$ Ultimately, there is simply no reliable evidence for this sign's consonantal value, and the most that can currently be said is that a vocalism in -a still seems probable.

\section{$\underline{2.13 * 83}$ 楚}

*83 has 25 certain examples at Knossos and Pylos. ${ }^{516}$ The most obvious type of variation displayed by this sign is that between the form with 'legs' on each side (seen at Pylos) and the form with a pair of legs on the right-hand side only (seen in the majority of

\footnotetext{
${ }^{512}$ The best possible evidence available to support the value wya is pe-re- ${ }^{*} 82-t a$, KN As $602 \sim p e-r e-w a-t a, \mathrm{KN}$ Vc(1) 183, PY An 192 (Doria 1972:48): both terms are MNs, but since pe-re-wa-ta at Knossos is recorded in the RCT and pe-re-*82-ta in the West Wing textile department, the two cannot be the same individual, so there is no firm evidence that they are the same name. There are no good alternations available for sya. ${ }^{513}$ As already demonstrated (p.134), there is no good reason to assume (with Melena 1983:263-6 and forthcoming:45) that a sign in $s w$ - would have originated as ${ }^{*} t w$ -

${ }^{514}$ Gallavotti 1959:164-5. Chadwick 1968a:64 cites a single example of Пíō̄ in Pindar as support for an

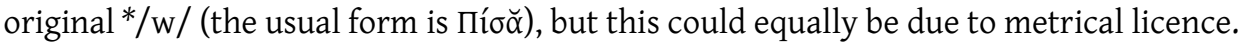

${ }^{515}$ The parallel example of ne-da-wa-ta =/Nedwātās/, related to the river Neda, at least involves both an MN found at Pylos (PY An 657.6, Jo 438.7) and a TN known in classical Messenia.

${ }^{516}$ The single possible example from Khania (e-[.]-*33-ro, where -[.]- is either -u- or -da-: Godart-Tzedakis 1991:130 and 1995:29; Olivier 1993:33) seems much more likely to be a slightly atypical example of -ko- (E. Hallager et al. 1992:72; E. Hallager 2011:420), since although the remaining strokes are compatible with the 'legs' of *83, there is simply not enough space to fit in the upper half of this sign. The possible example on the ISJ TH Z 847 ( $e^{*} 83-r a$ : Sacconi 2012:127) is even more doubtful; I prefer to read $e$-[.]-ra with CIV:129 (Judson 2013:80-1). There is no palaeographic basis for the suggestion that ${ }^{*} 83$ is a variant of $d w o \vec{\Delta} \vec{\Delta} \bar{\Delta}$ (L.R. Palmer 1963b:25, fig.3; Ruijgh 1972:101-2), since the two are clearly distinguished in several scribal hands (PY H1, H41; KN H103, H115, H141).
} 
Knossian examples, e.g. H118 and H117 below), but intermediate forms are also found (e.g. those with two centrally-positioned legs in KN H141 and H222, or the single RCT example).

\title{
Images removed for copyright reasons
}

\author{
PY En 74.1 (H1) $\quad$ Eo $276.2(\mathrm{H} 41)^{517}$
}

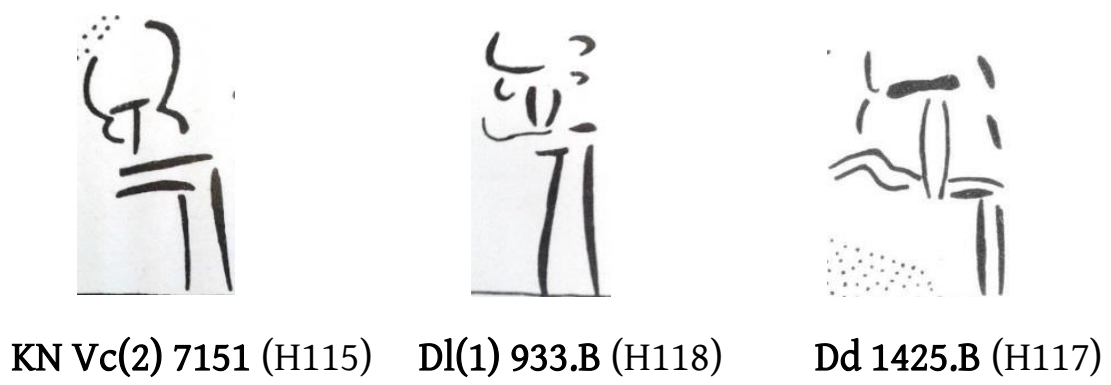

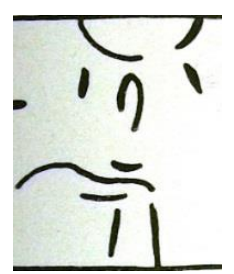

$\mathrm{Fp}(2) 363.2(\mathrm{H} 222)$

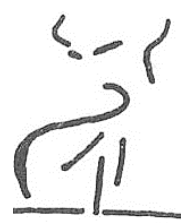

Sc $257.2(\mathrm{H} 124)^{518}$

Rather than being an example of the Pylos-type form, the RCT example seems to be similar to the H141/H222 form, but with the 'tail' at the left curving downwards. It seems probable that the two most common variants were created either through a process like

\footnotetext{
${ }^{517}$ Photos: author (NAM).

${ }^{518}$ All KN drawings: COMIK.
} 
this, with an original 'tail' being extended to form another 'leg', or via the opposite process, with the left-hand leg being simplified and reduced to a 'tail'; cf. PY H41's apparent simplification of the $\mathrm{H} 1$ form by drawing the right-hand leg much shorter than the left (and sometimes, as in the image above, with only one stroke instead of two).

Almost every other aspect of this sign can also vary, though some of these types of variation appear to be more significant than others: the shape of the strokes to the sides of the 'head' varies between a ' 3 '- or ' $\mathrm{S}$ '-shape, to simpler curved or straight strokes; ${ }^{519}$ the body of the sign can be made up of one or two strokes, ${ }^{520}$ as may the 'tail' if it is present at all. ${ }^{521}$ There is also a significant difference between the two sites at which *83 is found: the wide range of variation at Knossos, with no particular correlation between the various different forms of head, body, tail, and legs, contrasts with the use of a single basic form (with some minor variation between and within scribal hands) at Pylos. However, it is difficult to say to what extent this is due to the much larger number of scribes attested as using this sign at Knossos.

*83 has no known LA antecedent, so may have been created within LB, though a chance lack of attestations of *AB83 remains possible. The sign's distribution (in PNs and TNs in

\footnotetext{
${ }^{519}$ The ' 3 '-shape (which may also be drawn as two separate curves) is the most usual at both sites; the 'S'-shape is seen in KN H103 and H115, while a simplified form with two small straight strokes is seen on some examples in PY H1 and H41 and KN H117 (who all also use the '3'-shaped/small curves form) and in KN H222 and $\mathrm{H} 223$.

${ }^{520}$ One stroke: KN H115, H124, Uf 5763; two strokes: H103, H117, H118, H141, H222, H225, X 7592, and all PY examples. Whether these strokes are roughly parallel or slightly curved may vary considerably even within a single hand.

${ }^{521}$ No tail: KN H115 (Vc(2) 7517). One stroke: H115 (V(3) 479 v.2), H117, H118, H124, H225, Uf 5763, X 7592. Two strokes: H103, H117, H141, H222. Note the variation within both H115 and H117 in this respect.
} 
a range of scribal hands at two different sites) is perhaps more characteristic of an inherited sign than a newly-created one, although this is hardly conclusive.

\section{Prospects of decipherment}

*83 is attested only in various PNs, the two TNs $d a-{ }^{*} 83-j a(-d e)$ and $]^{*} 83-r e-j o-d e$, and the ethnic adjective (also used as a PN) based on the first of these TNs, $d a^{-}{ }^{*} 83-j o /-j a$, so its prospects of decipherment are in principle poor. ${ }^{522}$ Based on its distribution (mainly word-medial; initial in one or two terms $)^{523}$ it probably has a $C(C) V$ structure rather than a vocalic value. At Pylos, $r \mathbf{-}^{*} 83-0$ and $r$ - $^{*} 83-e$ are clearly the genitive and dative forms of the same MN, ${ }^{*} r u-{ }^{*} 83{ }^{524}$ The spelling of these case-endings (with $V$ signs rather than $C V$ ) implies that this name is an s-stem (although s-stem MNs generally appear to have datives in -i rather than - $e$ - see the list in Bartoněk 2003:263 - this could presumably be due to a chance lack of the latter form). The vowel of * 83 seems therefore most likely to be either -e (MN in /-ēs/; gen. /-e(h)os/, dat. /-e(h)ei/) or -o (MN in /-ōs/; gen. /-o(h)os/, dat. /-o(h)ei/). ${ }^{525}$

\footnotetext{
${ }^{522}$ Various highly implausible proposals are omitted from discussion here: e.g. $\mathrm{ko}_{2} / \mathrm{ka}_{2}$ (Georgiev 1955:9); $q \mathrm{o}_{2}$ (Georgiev 1956:81); kew (Docs²:244); da $a_{2}=$ dal (Melena 1974b:311-3); qe $e_{2}$ (Tovar 1958).

${ }^{523}$ Depending on whether ${ }^{*} 83\left[\right.$ ( KN Dv 8636) is the same name as * ${ }^{83-r e-t e ~(K N ~ U f ~ 5763 . b) . ~}$

${ }^{524}$ E.g. ru- ${ }^{*} 83-0$, ko-to-na (En 74.1) = 'R.'s plot of land'; pa-ro, ru-*83-e = 'from R.' (Eo 276.2-8). Both of these documents clearly refer to the same individual, since their lists of landholders are identical.

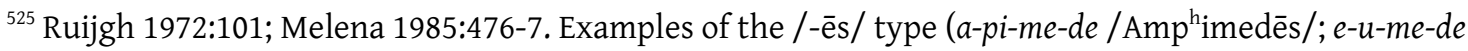
/Eumedēs/) are more numerous in Mycenaean than /-ōs/ (to-ro(-o) /Trōs, Trō(h)os/? appears to be the only suggested example: Bartoněk 2003:263-4). Note that even without taking the stem type into consideration, the spelling of $r u-{ }^{*} 83-0 /-e$ would appear to rule out a vocalic value in $-i$ or $-u$ given the lack of a glide (T. Meißner, pers. comm.).
} 
All of the values in -e or -o which were argued to be most plausible in theory for a sign invented in LB (e.g. further labialised or palatalised signs, ${ }^{526}$ or pto) were based on a criterion of usefulness in the context of Greek vocabulary and/or morphology, by analogy with signs such as $d w e$ and $d w o, r_{2}$, or pte (pp.46-7, 60-61); as remarked above, the distribution of*83 does not appear particularly compatible with an invention of this kind. Either *83 was invented under some other motivating principle - perhaps, in a similar way to two, in order to represent a particular recurring PN or TN (cf. the recurrence of $d a-{ }^{*} 83-j o /-j a$ and $\left.{ }^{*} r u-{ }^{*} 83\right)$, but in circumstances which led to its spreading to general use in a way that two did not - or it was in fact inherited, and the absence of *AB83 is due to chance. If the latter is the case, then the most plausible values in principle might be $\mathrm{pe}_{2}$ and $\mathrm{po}_{2},{ }^{527}$ palatalised values (rye, tye ${ }^{528}$ tyo) or $n w e / n w o^{529}$ are also possibilities, though perhaps less good ones due to the (infrequent) word-initial attestation of this sign. Without any good spelling alternations or identifiable Greek terms, however, these options cannot be narrowed down any further.

\footnotetext{
${ }^{526}$ Gallavotti 1958c:64 suggests that da-*83-ja da-te-we-ja (an ethnic adjective at Knossos) and that ${ }_{83}$ therefore = twe; however, as previously shown (pp.38-9) although sign *87's value of twe is not as secure as is often assumed, this (rather than kwe) is still the most likely value. In addition, the majority of examples of da-te-we-ja are in H103 (KN Ak(1) 612.C; Lc (1) 540.B; L(1) 594.b; Xe 5891) - who also has one example of the spelling with ${ }^{83}$ (]da- ${ }^{*} 83-j a-i, \mathrm{E}(2)$ 670.1). The assumption that */tw/ would have undergone affrication and twe therefore > sse or swe (Gallavotti 1958c:64; cf. Merlingen 1959:15 and 1963:164) has already been shown to be unfounded (p.134).

${ }^{527}$ The tentative suggestion by Witczak 2002-2003:125-6 that * 83 is $p e_{2}$ (to which Witczak assigns the value /be/: but cf. pp.29-31) is, however, based on little more than the desire to complete the $p_{2}$-series.

${ }^{528}$ Tentatively suggested as a possibility by Melena forthcoming:47-8.

${ }^{529}$ Melena 1985:476-8 argues for the value $n w e$ by comparison of $d a-{ }^{*} 83-j a$ to $d a-n w a$ (possible theonym, KN Gg 701); however, although offerings are sent to $d a-^{*} 83-j a$ (e.g. $\mathrm{Fp}(2) 363.2$, Fh 9067), this is more plausibly the name of a place where a sanctuary is located than of the sanctuary itself; cf. the appearances of the related ethnic adjective/PN, and also the fact that this is the location of a shepherd on Dv 1086.B. The suggestion $n w i$, based on similar grounds (Risch-Hajnal 2006:43), is excluded by the spellings of $r u-{ }^{*} 83-0 /-e$ (p.170, n.525).
} 


\section{$\underline{2.14 * 86}$}

*86 is the rarest of the undeciphered signs, with only 5 examples from Knossos and Pylos. Its simplest form (seen at Pylos) has a long vertical with two curves extending from the lower part of this stroke and coming to a point at the right.

Images removed for copyright reasons

PY Na 466

PY Na 1086 (both H1) $)^{530}$

The Knossian examples have a similar basic form, but with additional elements at the top of the vertical and/or around the pointed end; the H117 form also has an extra curved stroke extending from the top of the upright to meet the pointed end.

\section{Imageremoved for copyright reasons}

KN Ce $61.2(\mathrm{H} 124-\mathrm{B})$

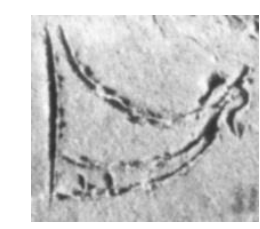

KN Dc 1117.B (H117) $)^{531}$

*86 has equivalents in both LA and $\mathrm{CH}$. CH 040 齿 can be a fairly elaborate depiction of a boat, but its simpler forms (those seen on clay documents rather than seals, shown below $)^{532}$ are closer to the greatly simplified LB *86 and AB86 $\nleftarrow$, which appear to represent only one half of the boat.

\footnotetext{
${ }^{530}$ Photos: author (NAM).

${ }^{531}$ Photos: author (HAM); MER.

${ }^{532}$ Drawings: CHIC.
} 


\section{Imageremoved for copyright reasons}

This may explain why some LA examples face the opposite direction to *86 (with the pointed end facing left): in the process of simplification which preserved only half of a form similar to $\mathrm{CH} 040$, it may have been possible to represent either half. ${ }^{533}$

\section{Imageremoved for copyright reasons}

\section{Prospects of decipherment}

The distribution of ${ }^{*} 86$, which is found in medial and final position, suggests a $C(C) V$ sign rather than a vocalic sign; the most probable values for a sign inherited from LA include those in $z^{-}, p_{2^{-}}, n w^{-}, r y^{-}$, and ty-, but no further information about this sign's probable value is available from its distribution. ${ }^{534}$ Since * 86 is found only in two MNs ( $m e^{*}{ }^{*} 86-$ ta and $\left.w a^{*} 86-r e\right)$ and one TN, $u-r a-{ }^{*} 86,{ }^{535}$ there is no possibility of any secure etymological

\footnotetext{
${ }^{533}$ Drawings: GORILA.

${ }^{534}$ The most that can be said is that its lack of initial attestations would be compatible with a palatalised sign or one in $n w^{-}$, but the sample size is evidently insufficient to draw any conclusions from this. There is no evidence as to its vocalic value; although the suggestion that me-*86-ta is an MN in /-tās/ is plausible, there is no reason why this must be /-ātās/ (as argued by Melena 1983:259).

${ }^{535}$ Melena forthcoming:48 regards $u-r a-{ }^{*} 86$ (PY Na 466) and $]^{*} 86(\mathrm{Na} 1039,1086)$ as probably not the same TN, presumably since TNs are not usually repeated in this series (Lejeune 1956:149-50). However, it would seem a rather large coincidence for two or three different TNs at Pylos to end in this very rare sign, so the restoration $u-r a-]^{*} 86$ on 1039 and 1086 seems probable.
} 
interpretations,${ }^{536}$ nor are any of these attested with alternative spellings. ${ }^{537}$ This is one of the signs least likely to be successfully deciphered.

\section{$\underline{2.15 \text { Conclusions }}$}

As stated in the introduction, the purpose of this chapter has not been to offer a decipherment proposal for any of the undeciphered signs, but rather to establish as firmly as possible what we can and cannot know about each individual sign given the current state of the evidence. In a minority of cases this extends as far as identifying a specific probable sound-value (*56: probably $\mathrm{pa}_{3} / \mathrm{p}^{\mathrm{h}} \mathrm{a} /{ }^{*} 65$ : very probably ju); for most of the signs this level of specificity remains impossible, but the range of probable values can be narrowed down to a greater or lesser extent through considerations of the signs' distributions and their inherited or invented status in comparison to the results of Chapter 1 . In two cases (*34 and *47), signs' distributions offer reasonably strong evidence for a vocalic value, corresponding exactly to the number of vocalic values identified as being plausible (ei and oi), although the value $z u$ also remains a possibility for *34. Of the probable $C(C) V$ signs, half can be further narrowed down by vocalic and/or consonantal value: ${ }^{*} 22$, probably $-i\left(z i, p i_{2}, n w i ?\right) ;{ }^{*} 49$ : probably $z$ - or $p_{2}-{ }^{*} 83$, probably $-e$ or $-0 ;{ }^{*} 18$, probably $-a,-e$, or $-o\left(p e_{2}\right.$ or $p o_{2}$ ??); *63, probably $-a,-e$, or $-o$. Although a much greater range of possibilities remains for the other signs $\left({ }^{*} 19,{ }^{*} 64,{ }^{*} 79,{ }^{*} 82,{ }^{*} 86\right)$, even in these cases the probable values are restricted to those shown in Chapter 1 to be most likely for signs inherited from LA or invented in LB, according to each sign's status.

\footnotetext{
${ }^{536}$ Proposals such as $n a_{2}$ (Georgiev 1955) or $\mathrm{sa}_{2}$ (Lejeune 1956:149, n.33) are both based on an etymological comparison of $u-r a-{ }^{*} 86$ to $u ̛ \eta \eta$, with different suggested suffixes.

${ }^{537}$ Melena 1983's comparisons of wa- ${ }^{*} 86-r e$ to various MNs at Knossos beginning wa-du- and me-* $86-$ ta to me-du[ ( KN X 7848.1) are not a secure basis for suggesting the value $d w a$.
} 
It should be repeated again that these results do not prove that any of these probable values must be attested, nor that any of these signs cannot have a different value; one of the other main conclusions of Chapter 1 was, after all, that LB signs have a wide variety of origins, not all of which fit into patterns which might be expected on theoretical principles. Nonetheless, alongside this chapter's more negative results in demonstrating that many previously proposed values (including some which have been relatively widely accepted) are improbable or lack any secure basis in the available evidence, considerable progress has been made in establishing the lines of investigation which are most likely to prove productive for future decipherment attempts - particularly in the case of new evidence coming to light, as it may well do, for instance, with further discoveries and publication from Ayios Vasileios. 
Chapter 3: The undeciphered signs as a palaeographic case-study

Chapter 2 demonstrated that although some progress can be made towards narrowing down the likely sound-values of individual undeciphered signs, in most cases an actual decipherment is not yet possible: these signs therefore cannot yet be read in the same way as the rest of the LB script. In this chapter, I use this group of signs to explore ways in which studying individual signs without necessarily being able to read them - i.e. through palaeographic analysis of their forms and the variation these display - can contribute towards our understanding of wider issues relating to the LB script and its context of use. In particular, I focus on two main areas: reconstructing the work of the Mycenaean scribes (including issues such as the operation of the palace administrative systems and methods of scribal training), and the use of palaeography as a chronological criterion for dating texts.

\section{1: The Mycenaean scribes: administrative work and scribal training}

Palaeographic analysis is, of course, the primary basis (alongside other features such as tablet formatting) for distinguishing different scribal hands, ${ }^{.538}$ and yet there has been comparatively little detailed discussion of either the methodology involved in such scribal identifications, ${ }^{539}$ or the possibility of employing this kind of analysis to further our understanding of the Mycenaean scribes' work and training. Although it has frequently been suggested that scribes' palaeographic similarities correlate to some

\footnotetext{
${ }^{538}$ Olivier 1967:26-33; Palaima 1988b:21-6.

${ }^{539}$ The most detailed discussion I am aware of is Palaima 1992-1993, and this concerns only a single, particularly controversial scribal identification.
} 
extent with relationships between them based on their administrative work or training, ${ }^{540}$ this has never been systematically investigated. ${ }^{541}$

A full investigation of the extent to which scribes' palaeographic similarities correlate to their administrative relationships is, evidently, far beyond the scope of this section; but in addressing this question with reference to the palaeography of the undeciphered signs, my aim is both to develop a methodological approach to this issue (to be further employed in a subsequent, more complete study of LB palaeography), and to explore the possible implications of my preliminary results for our reconstructions of scribal work and training. If palaeographic similarity does correlate with administrative relationships, that would imply that scribes learned how to write these signs, at least, ${ }^{542}$ by being taught by another scribe carrying out similar work in a kind of 'apprenticeship' ${ }^{.53}$ conversely, if no correlation can be seen between administrative relationships and palaeography, this would imply a very different situation in terms of scribal training (e.g. the existence of scribal 'schools'?) and/or administrative practices

\footnotetext{
${ }^{540}$ For instance, the RCT and NEP at Knossos are often said to have distinctive 'graphic styles', suggested by Olivier 1967:135 to be due to the presence of a 'master scribe' whose style was followed by their colleagues; Olivier also suggests that H117 and H119 in the East Wing, and H103 and H115 in the West Wing, are 'master' and 'pupil' due to their close similarity in both administrative work and palaeography. Palaima 1988b:188-9 similarly suggests that the three scribal classes at Pylos, to which scribes are assigned based on their palaeographic similarity to one of the three 'major hands', may be the result of these three individuals each being responsible for training a group of scribes (see also Palaima 2011:116).

${ }^{541}$ The most detailed explorations of scribal interrelationships have often been based on nonpalaeographic criteria: e.g. relationships between scribes at Pylos are discussed by Duhoux 1986 based on their orthographic variation, and by Kyriakidis 1996-1997 based on the content and location of their tablets.

${ }^{542}$ However, as discussed in Chapter 2 (see in particular pp.70-1), there is no strong reason to think that these signs differ significantly from the rest of the syllabary in any respect other than their frequency of attestation.

${ }^{543}$ In using terms such as 'apprenticeship' or 'school' (below) we must of course be aware of the possibility of anachronism; a Mycenaean system of either of these kinds may well have been very different in practice from similar systems in other cultures (cf. Duhoux 2011:95).
} 
(e.g. a greater amount of scribal mobility between different areas of the administration?).

Of course, since different sites vary considerably in their administrative organisation, ${ }^{544}$ it is possible that similar differences could exist in scribes' administrative interactions and/or training methods; each of the four sites at which scribal hands have been identified (Knossos, Pylos, Thebes, and Mycenae) will therefore first be discussed separately. For each site, a survey of the palaeography of the undeciphered signs will be conducted, focusing on the distribution of variant forms of each sign by scribal hand and location within the site; the reader is referred throughout to both the 'Undeciphered signs' spreadsheet and the fuller discussion of each sign's palaeography in Chapter 2 . The results of this study will then be analysed and their implications for scribal work and training at the site in question considered; finally, the results of all four sites will be compared and prospects for further research in this area considered.

\subsubsection{Knossos}

The administrative relationships between scribes at Knossos have already been reconstructed in some detail, in particular through the identification of the locations, administrative subjects, and scribal membership of different 'bureaux' and ‘departments' (Olivier 1967; Shelmerdine 1988). ${ }^{545}$ These may be non-specialised, dealing with a range of different subjects - for instance, the Northern Entrance Passage area (NEP: I2-3) $)^{546}$ and the Room of the Chariot Tablets (RCT) - or specialised in a particular

\footnotetext{
${ }^{544}$ E.g. on differences between Knossos and Pylos in particular, see Olivier 1984; Shelmerdine 1988 and 1999.

${ }^{545}$ See also studies of individual administrative areas: e.g. Foster 1977; Driessen 1999 and 2000.

${ }^{546}$ The NEP has been argued to be a 'pre-archive' (a medium-term record storage area: Driessen 1999); regardless of whether this is accepted, this deposit's lack of specialisation and relatively large number of scribes mean that merely being found in the NEP does not necessarily imply a close administrative
} 
subject or set of related subjects. Examples of the latter include the areas of the West Wing (WW) dealing with textiles (F6-15, F16-19, F21), aromatics (commodities such as spices and honey: E5, F3, F6-8, F15, F18, G1-3), ${ }^{547}$ and sheep (F1-2, F15); the E1 deposit (oil); and the East Wing (EW) sheep department (J1)..$^{54}$

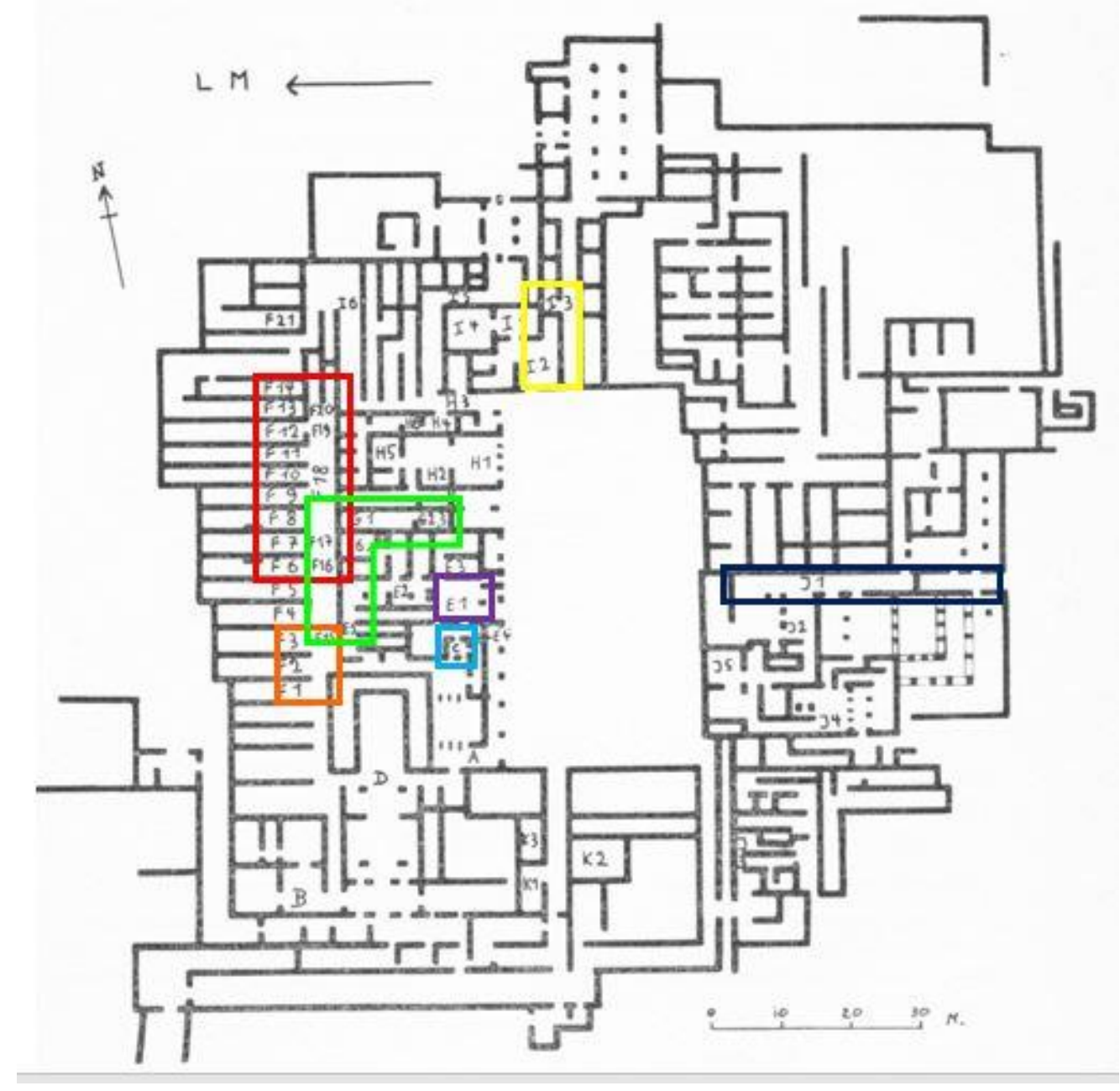

WW textiles - WW aromatics - WW sheep - E1 - RCT - NEP - J1 sheep ${ }^{549}$

connection between a pair of scribes, unless this is supported by further evidence (e.g. from subjectmatter).

${ }^{547}$ Note that the WW textile and aromatics departments overlap not only in geographical area, but also in the work of H103, who has produced tablets dealing with both subjects; these two departments therefore appear to be closely related.

${ }^{548}$ The various findspots in the EW are not as closely linked together as many of those in the WW. However, J1 and J2 share a pair of identical sealings and are not in fact clearly differentiated in terms of location (Firth 2000-2001b:243), while J2 and J3 are probably linked by H101 (Firth 2000-2001b:98); these are designated as a 'personnel bureau' by Olivier 1967:127.

${ }^{549}$ Map adapted from Olivier 1967:21. Outlines of administrative areas are approximate indications of the extent of tablet findspots only, and do not necessarily indicate the actual extent of the area in which these 
In general, the reconstruction of administrative relationships between Knossian scribes is based on the assignment of their tablets to one or more of these administrative areas. ${ }^{550}$ Within these areas, scribes may be more or less closely related by the specific subject-matter attested on their tablets, ${ }^{551}$ while some scribes may have worked in more than one area and therefore act as a link between those locations. For the purposes of this section I am most concerned with palaeographic variation within single departments or closely-related administrative areas, which is therefore demonstrably (approximately) contemporary. I shall, therefore, generally not address the issue of possible links between widely separated administrative areas; ${ }^{.52}$ discussion of the related, and highly controversial, question of these areas' chronological relationship will as far as possible be postponed until Section 2. This applies equally to the RCT, which stands the greatest chance of being chronologically separated from the rest of the Knossos tablets, but which for the purposes of this section will be treated simply as an administratively isolated deposit..$^{53}$

tablets (which mostly fell from an upper storey) were originally stored. Individual tablet findspots are generally referred to by the letter codes assigned by Olivier 1967:21-4 (as shown on the map), unless they form part of a larger administrative area (e.g. the NEP, the WW textile department), in which case they will be referred to as part of this area. Some smaller areas, such as the RCT (C) or the Arsenal (L), are also referred to by their names as these are more commonly used.

${ }^{550}$ Other kinds of administrative relationships may of course have existed; I focus on location and subjectmatter as the (potentially) most securely reconstructable of these possible relationships.

${ }^{551}$ Of course, this must to some extent be dependent on chances of attestation; that two scribes do not appear to share any similar administrative topics in the extant tablets does not prove the absence of a working relationship (they might have interacted outside of the time-period represented by the surviving tablets; in tablets from this period which do not survive; or in surviving tablets which we are unable to identify as related due to their condition and/or our own limited knowledge of the relevant subject). In attempting to reconstruct the palatial administrative systems, it is necessary to use the positive evidence available while refraining from placing too much weight on an absence of evidence.

${ }^{552}$ For instance, between the WW, NEP, and EW: on this issue, see Firth 1996-1997 and 2000-2001b.

${ }^{553}$ It has been suggested (most recently by Duhoux 2011; see also Chadwick 1967 and 1968b) that the RCT is a scribal training area. However, the best evidence for this is two tablets (Ce 50 and V(1) 114) which each have the same text on their recto and verso and might, therefore, be practice texts (Duhoux 2011:96-101); $\mathrm{V}(1) 114 r$. and $v$. may also be written by two different hands, though the identification of these as 'master' and 'pupil' (Duhoux 2011:99) is highly subjective, and since a high level of intra-scribal variation is a prominent characteristic of the RCT (Driessen 2000:97), it remains possible that two sides were written by 


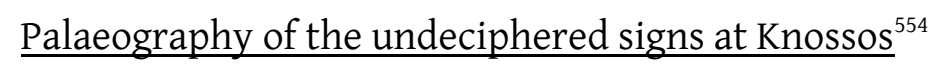

*18 $\stackrel{\text { "in- }}{ }$ (pp.71-2)

This sign appears up to 10 times at Knossos; the scribes who use it are distributed as follows:

WW textile department: H103 x1 (F14)

NEP: $\mathrm{H} 118 \times 1$ (I3)

J1: $\mathrm{H} 117$ x4+1

Arsenal: $\mathrm{H} 132 \mathrm{x} 1$

Unknown: X 7739.ḅ, 7?9ִ4ִ̣ (-) $)^{555}$

All the examples from known findspots - which include most of the major administrative areas of the place - are of Type 1 (H117: Type $1 \mathrm{a}$ and $1 \mathrm{~b}$; all others: Type 1b); the two of unknown hand and findspot are the only known Type 2 examples.

the same hand (see Olivier 1967:97; Driessen 2000:203, n.16). This hardly seems sufficient evidence to support the hypothesis that all of the c.600 RCT documents - most of which appear perfectly able to function as administrative records - are in fact training exercises. I therefore follow Driessen 2000 (cf. also Camera 1968) in assuming this deposit to be (primarily) an administrative one.

$554 *_{56}$ is excluded from this and all following sections since it displays no significant variation (p.107). In the lists of (possible) attestations of each sign, an underdot indicates an uncertain reading, and a question mark an uncertain findspot; uncertain scribal attributions are indicated separately (e.g. tablets attributed to 'H101?' are listed after those attributed to H101). Tablets whose original findspot is not recorded, but which have been attributed to a particular findspot with a reasonable degree of probability by the work of R.J. Firth and J.L. Melena (i.e. those underlined in the spreadsheet), are included under that findspot without comment; tablets whose attribution is more doubtful (underlined plus question marks in the spreadsheet) are generally included under 'unknown findspot' and discussed where necessary. See Firth 1996-1997 and 2000-2001b; Firth-Melena 1998-1999, 2000-2001, and 2008.

${ }^{555}$ I regard these as very probable examples: p.71, n.175. 

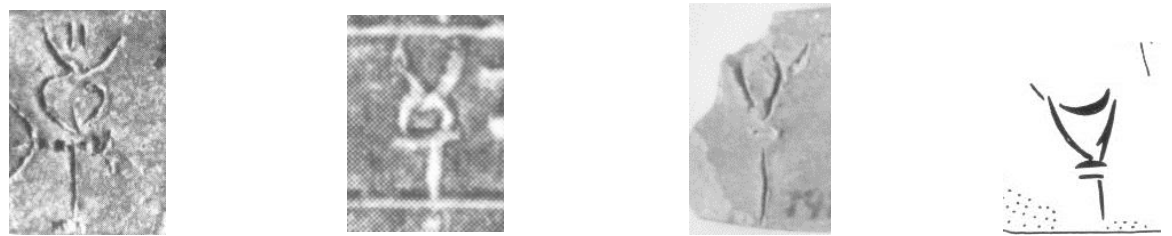

Type 1a: Db 1211.B (H117) 1b: Ap 639.4 (H103), Dl(1) 950 (H118) Type 2: X 7739 (-) $)^{556}$

*19 $\stackrel{1}{\AA}(\mathrm{p} .76)$

This sign has only 2 (probable) examples at Knossos:

RCT: H124-A x1

Unknown: X $9014(-)^{557}$
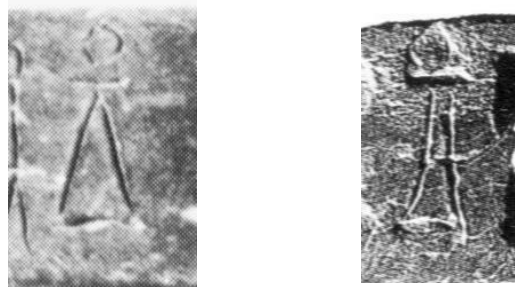

KN Ag 90 (H124-A) KN X $9014(-)^{558}$

These signs are essentially fairly similar in form, apart from X 9014's cross-stroke and slightly curved sides.

$\underline{*_{22 / \mathrm{CAP}}}{ }^{\prime}(\mathrm{pp} .84-6)$

This sign has 131 certain/possible examples at Knossos, distributed as follows:

\footnotetext{
${ }^{556}$ Images: COMIK, except 950 (MER).

${ }^{557}$ There is no strong evidence to suggest that X 9014 originates from the RCT (it belongs to a batch which includes fragments probably from the RCT, but also from E1, the WW, and the NEP: Firth-Melena 2008:297), and therefore none to suggest that ${ }^{*} 19$ was exclusively used in this location at Knossos. ${ }^{558}$ Photos: MER.
} 
WW textile department: H103 x3 (F10, F10-11?, F14); H108 x1 (F7); H116? x1 (F14?)

WW aromatics department: H223 x1 (F3) $)^{559}$

WW sheep bureau: H121 x2, H217? x1 (F1)

E1bis: C 394 v.2(-)

H3: F(3) 741.2-4, 8242.1.2? ${ }^{560}(-)$

RCT: H124-B x1, H124-C x1, H124 x9+1

B2: H101 x4

NEP: H107 x10 (I3); H111 x6 (I3); H112 x6 (I3); H112? x5 (I3); H120 x1 (I3); H122 x1 (I3);

H125 X2 (I3; I2-3?); F(2) 841.2; G 820.3; C 954.1.2.2.3.3 and v.1, 6021.1 (-; I3)

J1: H117 x23; Mc 1508.A.B, 5187.B; Wb 5665 (-)

J3 (or J2): H105 x1

Arsenal: H132 x13+2; H133? x1; Xf 8835 (-)

Little Palace: $C<1902.1 .2>(-)$

Unknown: 10+2 examples: C 5699.1.2, ${ }^{561}$ 5730.B.Ḅ, 7054, 7059.B, 7088 [1+1]]; X 5784, 7553, 7655, 7741.a, $9691^{562}(-)$

Both single- and double-stalk forms of $*_{22}$ are found, the former being slightly more common (42 examples) than the latter (17 examples): ${ }^{563}$

\footnotetext{
${ }^{559}$ NB: hands numbered in the 200's (the 'secondary hands') are not, strictly speaking, identified scribes, but designations for a group of tablets which belong together palaeographically but which cannot certainly be either distinguished as the product of a separate hand or attributed to an identified one (Olivier 1967:40). Forms written by secondary hands are, however, included in this section for the sake of observing variation within administrative areas.

${ }^{560}$ This is probably part of the same tablet as $\mathrm{F}(3) 741$ (KT $\mathrm{T}^{5}$; COMIK).

${ }^{561}$ Suggested by Firth-Melena 2000-2001:348 to originate from the RCT, but this is very uncertain, particularly since the tablet has no scribal attribution.

${ }^{562}$ Firth-Melena 2008:300 state that this is part of a group of fragments mostly originating from the NEP, but it is not certain whether this applies to this fragment.

${ }^{563}$ Plus three examples of uncertain form due to the lower part of the sign being missing: Db 5715.b (H117);

Xf 8835, X 7675 (-).
} 
Single-stalk forms:

WW textiles department: H103 x3

WW aromatics department: $\mathrm{H} 223 \mathrm{x} 1$

WW sheep bureau: H121 x2, H217? x1

H3: F(3) 741.2-4, 8242.1.2? (-)

RCT: H124-C x1, H124 x1 (Xd 7808)

NEP: H120 x1; G 820.3

J1: H117 x18; Wb $5665(-)$

J3 (or J2): H105 x1

Arsenal: H132 x1; H133? x1

Unknown: C 5370.B; X 7553, 7655, 7741.a, 9691

Double-stalk forms:

WW textiles department: H108 x1; H116? x1

RCT: H124 x1 (Xd 131) $)^{564}$

B2: $\mathrm{H} 101 \mathrm{x} 4$

NEP: H122 x1; H125 x2; F(2) 841.2 (-)

J1: H117 x4 (Dv 1216.B; Da 2027; De 5032.B; Dn 1093.1); Mc 1508.B (-)

Unknown: X $5784(-)$

The only other significant form of variation in this sign at Knossos (where variation in the form of the head is not diagnostic) is the presence or absence of a small stroke underneath the head (also seen in AB22 $\uparrow$ ): examples with this stroke are only certainly found in the RCT and NEP.

\footnotetext{
${ }^{564} \mathrm{NB}$ : this example is the only double-stalk form to be identical to $\mathrm{CAP}^{\mathrm{f}}$, with diagonals that diverge rather than meeting at the bottom.
} 
RCT: H124-B x1, H124 x2 (Ce 7516.1; Xd 7808)

NEP: H107 X10, H125 x1, ${ }^{565}$ F(2) $841.2(-)$

unknown: C 7088; X 7655 (-)

*34

This sign has 9 examples at Knossos:

RCT: H124-G X1; H124-I x1; H124? x1

NEP: H114 x2+1? (I2; I3+I3bis; I2/3?) ${ }^{566}$

J1: H117 x2, H117? x1

All examples from the RCT and by H117 are *34 $\alpha$, while H114's examples are all *34 $\beta$.

Knossos also has the full range of variation in the elements at the centre of the

semicircle: the RCT forms are the simplest (with no central element, or a small curve) and the closest to AB34 $<$ < H117 shares the latter form, but also has a more elaborate majuscule; H114 has a complete circle, like the mainland forms.

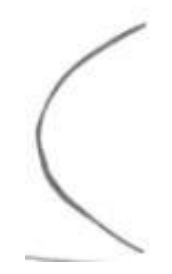

KN Sc 235 (H124-G)

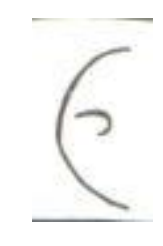

Dv $1239.1(\mathrm{H} 117)$
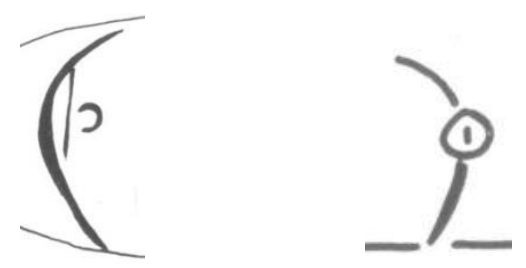

Da 1253.B (H117) Ld $787(\mathrm{H} 114)^{567}$

\footnotetext{
${ }^{565} \mathrm{~V}(5)$ 1002.B; note that this hand's other example of *22 (V(5) 756.B) lacks this feature.

${ }^{566} \mathrm{Ld}(2)$ 8192, whose original findspot is not recorded, was assigned to the NEP by Firth 2000-2001b:226, as all of H114's Ld(2)-series with known findspots are from this location; however, Firth-Melena 2008:306 assign it to F14. In the absence of any explanation being given for this change, I continue to assign this tablet tentatively to the NEP.

${ }^{567}$ Drawings: COMIK.
} 
苔简( pp.100-1)

This has 15-22 certain/possible attestations at Knossos, distributed as follows:

WW textile department: H115 x1 (F7)

A: $\mathrm{H} 138 \mathrm{x} 2$

E1: H141 x4+2; H141? x3

RCT: H124-G x1; H124-S x1; H124-7 x2; H124 x2

I1: H224 x1

Unknown: X 8775 v., $92599,9849^{569}$

The simpler form of * 47 (with 2-4 small curved strokes around the centre; this is closest to AB47 $\not)$ is found in the WW (H115, H138: below left) and I1 (H224), and perhaps on the three possible examples of unknown findspot; the more elaborate form (with a circle surrounded by small strokes) is found in the RCT (all examples) and E1 (H141: below right). $\mathrm{H} 115$ and $\mathrm{H} 138$ are the only scribes to write this sign without the end-strokes on the diagonals (which are also not seen in LA).

\section{Imageremoved for copyright reasons}

$\mathrm{Fp}(1) 1.9(\mathrm{H} 138)$

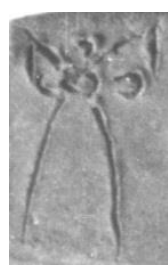

Fh $393(\mathrm{H} 141)^{570}$

\footnotetext{
${ }^{568}$ This includes Fh 462, originally recorded as found in F3; since these two locations were excavated within a day of each other (Driessen 1997:120) and since 462 is now joined to 5470, recorded as found in E1 (Firth-Melena 1998-1999:123, n.30), this is most probably a recording error; I follow Driessen, Firth and Melena in assigning this tablet to E1. See pp.195-6.

${ }^{569}$ Firth-Melena 2008:301 state that this is part of a group of fragments mostly originating from the NEP, but it is not certain whether this applies to this fragment.

${ }^{570}$ Photos: author (NAM); MER.
} 
*49 $\mathbb{\mathbb { A }}(\mathrm{pp} .104-5)$

This sign is attested up to 6 times at Knossos:

WW textile department: H103 x2 (F14)

J1: H117 x1+2; H119 x1

The only form of palaeographic variation seen in this sign is the presence or absence of two small strokes near the bottom of each vertical: these are present in all J1 examples (H117 and H119) as well as in AB49 $\mathbf{j} \mp$, but absent in H103. However, as the former are all majuscule and the latter minuscule, it is not certain whether this is a diagnostic feature.

$\underline{* 64}[\mathrm{Q} .128-9)$

This sign has 2 examples at Knossos:

RCT: H124-F x1

Unknown: X $5516(-)$

There is no particularly good reason to assign X 5516 to the RCT rather than any other part of the palace, and therefore *64 cannot be regarded as exclusively used in the RCT (pp.130). There is no significant palaeographic difference between these two examples, which both have the small strokes on the ends of the verticals.

*65/FAR ऋ (pp.135-8)

This sign has 24-8 examples at Knossos: 
WW textile department: H103 x3 (F14; G1)

A: H139 x13; Fs 3.B.B (-)

RCT: H124-E x1; H124-4 x1; H124 x2; H124? x1

J1: H117 x2

Unknown: X 5509, 7̣5̣5̦3̣, 9519 (-)

Type 1 is the most common form of*65/FAR at Knossos, found in almost all the examples listed above; although there is some variation within this group, it cannot be neatly divided into sub-groups: for instance, H124-E and H117 have a much more open diagonal than the others, but H117's base is closer to that of H103 in lacking a stroke at the bottom; a form with a branching end to the diagonal is found in H139 and on Fs 3.B (A; -), but the latter also has a second, unique form of this sign.

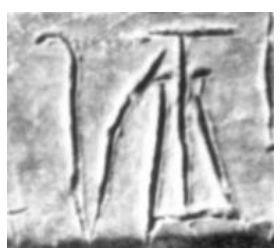

Type 1: U(1) 49 (H124-E)

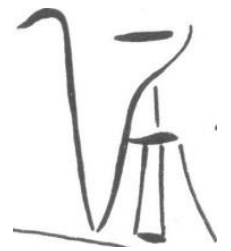

Dc 1403.B (H117)

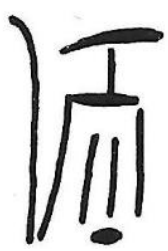

$\operatorname{Od}(2)$ 714.a (H103)

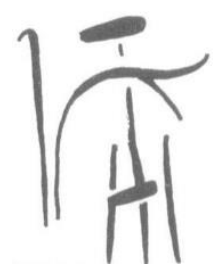

Fs 20 (H139)

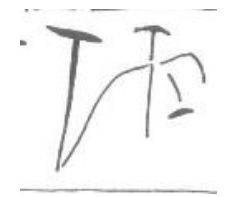

Fs 3.B (FAR)

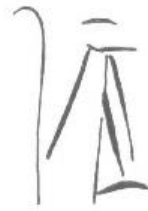

Fs 3.B $\left({ }^{*} 65\right)^{571}$

${ }^{571}$ Photo: MER; drawings: COMIK. 
The only Type 2 example is one from the RCT (H124-4: $\mathrm{Xd} 105):{ }^{572}$ this very simplified form is more comparable to some mainland forms, and to AB65 h, than to the other examples at Knossos.

*79

This sign has only two examples at Knossos:

WW textile department: $\mathrm{H} 103 \mathrm{x1}$

J1: H117 x1

H103 has the simplest form of this sign, a plain oval; H117 has the most complex, with a stroke in the middle and small strokes around the outside (this is the form closest to $\mathrm{AB79}$ 泎 and $\mathrm{CH} 005$.

$\left.{ }_{82}\right\}_{2}^{2}(\mathrm{pp} .162-4)$

This sign has only 2-3 examples at Knossos:

WW textile department: $\mathrm{H} 103 \mathrm{x} 1$

J1: H117 x1

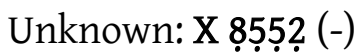

H103 has a Type 1 form (with a central ' 3 ' shape), similar to AB82 1 隹; H117's form is intermediate between this and the simpler Type 2 seen on the mainland..$^{73}$ These

\footnotetext{
${ }^{572}$ Drawing: COMIK.

${ }^{573}$ The traces on $\mathbf{X} 8552$ are compatible with either an H117-type *82, or ku.
} 
intermediate and Type 2 forms probably originated from a simplification of the Type 1 form.

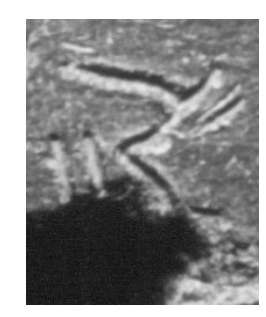

$\operatorname{As}(1) 602.4(\mathrm{H} 103)$

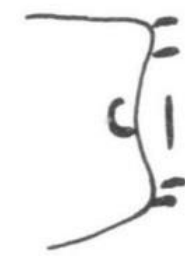

Dc 1154.B (H117) $)^{574}$

*83 录 $($ pp.167-9)

This sign has 16 certain examples at Knossos:

WW textile department: H103 x1 (F14); H115 x2 (F6/7; F13/14?) ${ }^{575}$

WW aromatics department: H223 x1 (F3)

E1: H141 x1; H141? x1; H222 x1

RCT: H124 X1

NEP: H118 x1; H225 x1 (I3)

J1: H117 x4

Unknown: Uf 5763.b; X 7592 (-)

It is thus used in all the major administrative areas of the palace. In addition, there are several examples of scribes working in the same department whose sign-forms can be compared.

\footnotetext{
${ }^{574}$ Photo: MER; drawing: COMIK.

${ }^{575} \mathrm{Vc}(2) 7517$ is attributed to F13/14 by Firth 2000-2001b:232, but only to the WW by Firth-Melena 2008:303.
} 
As shown in Chapter 2, *83 cannot be straightforwardly classified into variant forms, since individual aspects of the sign can vary independently of each other. The attestation of different forms of each part will therefore be listed separately.

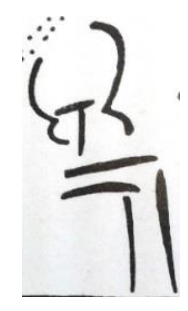

KN Vc(2) 7151 (H115)

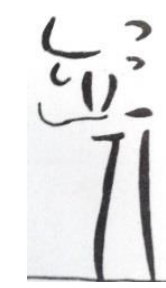

Dl(1) 933.B (H118)

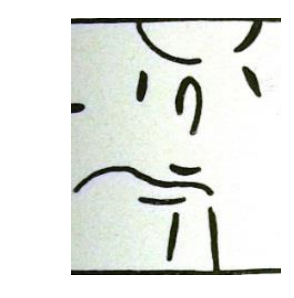

$\mathrm{Fp}(2) 363.2(\mathrm{H} 222)$

Fp(2) $363.2(\mathrm{H} 222)$

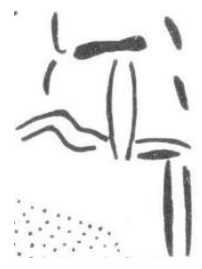

Dd 1425.B (H117)

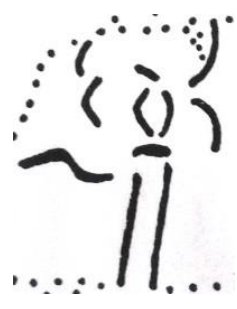

Fh 9067 (H141)

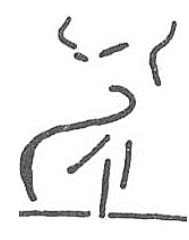

Sc $257.2(\mathrm{H} 124)^{576}$

Right-hand 'legs': WW textile department: H103, H115; E1: H141?; J1: H117; NEP: H118, H225

Central 'legs': E1: H141, H222; RCT: H124

Uncertain: WW aromatics department: H223

'Tail' to the left:

None: WW textile department: H115 (Vc(2) 7517)

One stroke: WW textile department: H115 (V(3) 479 v.2); RCT: H124; J1: H117; NEP: H118, H225 (also Uf 5763; X 7592)

Two strokes: WW textile department: H103; E1: H141, H222; J1: H117

Uncertain: WW aromatics department: H223

${ }^{576}$ All KN drawings: COMIK. 
Shapes at each side of the 'head':

'3'-shapes/two small curves: E1: H141; RCT: H124(?); ${ }^{577}$ J1: H117 (Dc 1419.B; Dv

1086.B, 8636.B); NEP: H118 (also Uf 5863.b; X 7592)

'S'-shapes: WW textile department: H103, H115

small straight strokes: WW aromatics department: H223; ${ }^{578}$ E1: H222;

J1: H117 (Dd 1425.B)

Uncertain: NEP: H225

Body:

One stroke: WW textile department: H115; RCT: H124 (also Uf 5763)

Two strokes: WW textile department: H103; E1: H141, H222; J1: H117; NEP: H118, H225 (also X 7592)

Uncertain: WW aromatics department: H223

*86

This sign has 2 examples at Knossos:

RCT: H124-B x1

J1: H117 x1

The forms of both examples are fairly similar (especially compared to the simpler Pylian forms and AB86 $\nleftarrow$ ), though the RCT form has more elaboration around the top, and the H117 form has an extra curve from the top to the right-hand end (and is therefore more similar to CH 040 : pp.172-3).

\footnotetext{
${ }^{577}$ This form is difficult to classify as the two sides differ considerably.

${ }^{578}$ Three small strokes and one larger curved stroke extending to the middle of the head; but overall this is more similar to the H222 form than the ' 3 '-shaped/small curves forms.
} 


\section{Imageremoved for copyright reasons}

KN Ce 61.2 (H124-B)

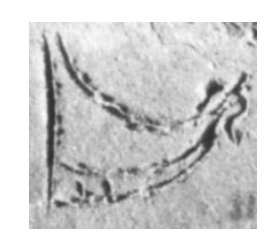

KN Dc 1117.B (H117) $)^{579}$

\section{Discussion}

The best opportunity to explore a possible correlation between palaeography and administrative work is provided by those signs that are attested in both administratively-related scribal hands and apparently unrelated ones. The most secure examples of this are provided by ${ }^{*} 22,{ }^{*} 49$, and ${ }^{*} 83$, which will therefore be discussed first; most of the other signs' evidence is less certainly significant due to their circumstances of attestation, though still worth examining in this respect. ${ }^{580}$

The distribution of $* 49$ appears to correlate very well to administrative relationships: H117 and H119, who worked closely together in the J1 sheep department (H119's Dk(2)series tablets refer to the same flocks listed by H117's Da-Dg records), ${ }^{581}$ write this sign with the strokes on the ends of the verticals, while H103 (WW textile department) does not. In fact, as mentioned above (p.177, n.540), Olivier 1967:135 identifies H117 and H119 as 'master' and 'pupil', based on their close administrative relationships as well as the similarity of their palaeography. However, this is a very small sample size and, as remarked above, it is not entirely clear how diagnostic this variation is. ${ }^{582}$

\footnotetext{
${ }^{579}$ Photos: author (HAM); MER.

$580 * 19$ and *64 will not be discussed as they display little palaeographic variation and have only two examples, of which only one in each case has a known findspot.

${ }^{581}$ See Killen 1964:9-11. It is, however, unknown whether H119 used H117's tablets directly in compiling the $\mathrm{Dk}(2)$ records (Melena forthcoming:23), or whether they merely shared a source of information (Olivier 1967:135, n.54).

${ }^{582}$ It is also possible, but not confirmed, that H119 is in fact connected to the WW textile department. As well as the $\mathrm{Dk}(2)$-series, H119 is responsible for the Pp-series tablets, found in the WW in F7a: this indicates
} 
The use of the small extra stroke under the head of *22/CAP similarly appears to correlate to administrative work to some extent, since this feature is restricted to two administrative areas, the RCT and NEP. However, in neither case is this feature characteristic of the whole deposit: there are four scribes in the NEP (H111, H112, H120, H122) who do not use this feature, and 9 of the 12 RCT examples of *22/CAP also lack it. Nor is there evidence to link H107 and H125 particularly closely; H125's tablets are all Vseries, i.e. personnel, while H107's are livestock tablets in the B- and C-series (and the single tablet by an unknown hand with this form of *22 is in the $F(2)$-series, i.e. dealing with rations). In a 'non-specialised' department featuring almost 30 different scribes, ${ }^{583}$ this is not sufficient evidence to demonstrate a close working relationship. Compare the distribution of the single- and double-stalk forms of *22/CAP: the scribes who only write the former are found in all three of the main WW administrative areas (dealing with textiles, aromatics, and sheep), the EW (J3 or J2), the NEP, and the Arsenal, while the scribes who only write the latter are found in B2/3, the WW textile department, and the NEP; the RCT has both, as does H117 in J1 (where unattributed examples of both forms are also found). It is immediately apparent that the largest departments, including the two non-specialised departments (the RCT and NEP) as well as the WW textile department and the J1 sheep department, all have both forms present. Moreover, closely linked individual hands can differ in this respect: for instance, H103 and H108, who both worked in the WW textile department and even wrote tablets in the same series (Ak-). It

\footnotetext{
a lower level than other tablets from F7, so that (unlike the others) the Pp tablets probably originate from the ground floor. Driessen 1997:125-7 argues that since the Pp tablets must therefore be of earlier date than the blockage of the Long Corridor, they are also earlier than the upper-floor WW tablets; but in the absence of firm evidence for the date of this blockage, it seems more plausible that this would have taken place at whatever point the whole WW fell into disuse (cf. Raison 1993:120-1, 161; Boardman 1963:33; Driessen 1990:121-2). However, this remains disputed, and in any case the subject of the Pp-series, ${ }^{*} 168+S E$, is obscure (Vandenabeele-Olivier 1979:281-2).

${ }^{583}$ Driessen 1999:213-14.
} 
is clear that the use of the single- and double-stalk forms is not correlated to administrative relationships.

*83 is found in at least two scribal hands in 3-4 administrative areas - the WW textile department (H103 and H115) and aromatics department (H223; H103 also worked in this department), E1 (H141 and H222), and the NEP (H118 and H225) - and therefore offers a particularly good opportunity to test this correlation. H141 and H222 are both found only within the E1 deposit, and so appear to have an extremely close administrative relationship; they also share a unusual form of *83 (with centrally-positioned 'legs'), paralleled only in the single RCT example (though there is also an E1 example of the more usual right-hand 'legs' form, ascribed to H141?: Fh 365.a), as well as corresponding in the form of the 'tail' (one stroke); the form of the 'head', however, differs significantly, as does the overall construction of the sign (the various features are quite differentlyproportioned). Similarly, H103 and H115 (which have also been suggested to be 'master' and 'pupil': p.177, n.540) share elongated 'S'-shaped forms to either side of the 'head', which are not seen in any other hand; however, in H115 the 'body' consists of one stroke, as does the 'tail' (if present), while in H103 both have two strokes. In some respects, therefore, H103 is more similar to H117, to whom they are not directly related; moreover, H223 (found alongside H103 in the aromatics department) does not share H103 and H115's characteristic form of the 'head'.

It also seems probable that rather than being an isolated deposit, ${ }^{584} \mathrm{E} 1$ (which deals with distributions of oil) is in fact administratively connected to (if not part of) the aromatics

\footnotetext{
${ }^{584}$ Driessen 1990:69-71 and 1997:120 argues that the E1 tablets are significantly earlier in date than the majority of the WW tablets. The archaeological evidence for this is, however, debated; according to Driessen, excavation records show that the floor of this room was significantly lower than those of the
} 
department. Although there are no scribal links between E1 and any other part of the palace, ${ }^{585}$ there is a probable/possible palmprint match (L DELTA) between some of H141's tablets and H110's Ch 7065, probably from I3 (Sjöquist-Åström 1991: Table II; Firth 2011:85-7); more importantly, an individual called ku-pi-ri-jo - probably a 'collector' in the perfumed oil industry (Killen 1995) - plays a very similar role in distributions of both oil in E1 (Fh-series) and spices in the aromatics department (Ga- and Gg-series). ${ }^{586}$ Although it is not impossible that ku-pi-ri-jo in E1 and in the aromatics department could be different people (perhaps related, if they are indeed separated by a generation, as Driessen 1997:120 argues), it seems much more probable that they are the same individual, and that the E1 tablets are therefore (approximately) contemporary with, and administratively linked to, the aromatics department. ${ }^{587}$ Regarding *83, we thus have five scribes within the broadly linked administrative areas of the WW showing almost the complete range of forms of this sign seen at Knossos.

The NEP forms of *83 likewise differ considerably in some respects: e.g. H118 has the ' 3 'shape/small curves to each side of the head, while H223 has small straight strokes (though it is difficult to analyse these further since H223's example is only partly preserved, and H225's idiosyncratic form appears to be due to space limitations; there is also no reason in terms of subject-matter why these scribes should be particularly closely related). ${ }^{588}$ Overall, there is once again no correlation between administrative and palaeographic relationships.

\footnotetext{
surrounding rooms, but Firth 2000-2001b:204 disputes this on the grounds that it assumes a greater precision in the measurements recorded during excavation than may be justified.

${ }^{585}$ See p.186, n.568.

${ }^{586}$ See Godart 1968 and 1969; Foster 1977. Although Godart and Foster differ in their interpretations of the role of ku-pi-ri-jo, both treat E1 as part of the wider department.

${ }^{587}$ As is explicitly concluded by Godart 1968:70 and 1969:65, and assumed by Foster 1977 and Killen 1995.

${ }^{588} \mathrm{H} 118$ has written a large number of D-series tablets; H223 has only certainly written two G-series tablets, and $\mathrm{H} 225$ one $\mathrm{V}$-series and one $\mathrm{X}$-series.
} 
*47 and *65 both have examples by hands which may be administratively linked depending on the interpretation of the relationship between the aromatics department and A (the Clay Chest). The A tablets deal with offerings of various commodities, including oil and honey (H138: Fp-, Gg-; H139: Fs); Olivier 1967:125-6 suggests that it may be related to the aromatics department, as was argued above for E1. Although there are no secure links between A and other deposits, ${ }^{589}$ its thematic similarity to the aromatics department (on which see Foster 1977; Gulizio 2008) make an administrative relationship between the two plausible (though evidently not certain), and it is worth examining the palaeographic implications such a relationship would have.

Within the A tablets, two main forms of *65/FAR are found (both belonging to Type 1): the more common, with a branching end to the diagonal, is found in H139 and on the unattributed tablet Fs 3, raising the possibility that this form is characteristic of this one deposit. However, Fs 3 also has a completely unique form, perhaps due to the unusual attestation of both *65 and FAR on the same tablet (and note that the branched form is also paralleled at Pylos). In addition, if A does form part of the broader aromatics area, then both the branched and unbranched forms are found within this area (the latter in H103). These forms could also be compared in terms of the shape formed by the upright and diagonal, which may be a very open 'V' shape (seen in, e.g., H124-E and H117) or a

\footnotetext{
${ }^{589} \mathrm{X} 453$, attributed to H138?, was found in F3; this findspot, and the occurrence of the 'collector' name we-we-si-jo-jo, link this tablet to the textile department, suggesting that H138 could, like H103, have worked in both the textile and aromatics administrations; however, this attribution is not certain. Olivier 1967:845 suggests that $\mathrm{H} 139$ could actually be identified with $\mathrm{H} 140$ (the author of three Gg-series tablets from G1), but again this is apparently not secure enough to classify these as a single hand. Firth 2000-2001b:205 sees a possible link to E1 via the month names, noting the attestations in E1 of ka-ra-e-i-jo (Fp(2) 354) ( ka-ra-eri-jo, Fp 6.1 et al.) and de-u-ki-jo (Fh 5502) ( de-u-ki-jo-jo, Fp 1.1). However, it is not generally agreed whether ka-ra-e-i-jo is in fact a variant of ka-ra-e-ri-jo or the name of a recipient (DMic q.v.), while de-u-ki-jo has an uncertain reading and would be in an unusual position for a month name, directly following an MN. Even if the same month names were attested, this could not conclusively prove the deposits dated from the same year, nor provide a certain administrative link between the two.
} 
much narrower one: the latter is shared by H103 and H139, but both forms on Fs 3 have the more open shape. Of the two certain examples of *65/FAR in the RCT, that by H124-E is of the regular Knossian Type 1 (as the uncertain examples would probably also be), but the one attributed to H124-4 is the only Type 2 example found at Knossos.

Maintaining the assumption that A is part of the aromatics department, ${ }^{*} 47$ would be found in two relatively closely related hands, H138 (A) and H141 (E1), as well as in the more indirectly related H115 (found only in the textile department, but this is closely connected to the aromatics department via H103). H115 and H138 are palaeographically close in the lack of the end-strokes seen in all other hands, as well as in using the simpler form of the central element; however, H141 differs in both respects, with the more complex central element and the end-strokes. The two WW scribes who are most likely to be closely related thus use completely different forms of *47, and the greatest similarity in the form of this sign is seen between a pair of scribes who have much less chance of being related. In the RCT, by contrast, all of the examples of * 47 are of the same form (with the more elaborate central element, and end-strokes): note that these are probably by at least two different hands (H124-G and H124-S have probable examples; certain examples are attributed to H124-7 and H124).

The same is the case for the RCT examples of $*_{34}$, which are all *34 $\alpha$, and of the simplest forms (with no central element, or only a small curve in the middle); these are by at least two different hands (attributed to H124-G, H124-I, and H124?). There are, however, no examples of more than one scribe from the same administrative area outside of the RCT writing *34: the fact that H114 (NEP) and H117 (J1) use different forms of *34 may or may not be linked to their lack of any administrative connections, but without further comparanda this is not certainly significant. The same goes for *79, *82, and *86, which 
also have different forms in hands with no known administrative connection $\left({ }^{*} 79,{ }^{*} 82\right.$ : H103 and H117; ${ }^{590}$ *6: H124-B and H117). There are, likewise, no examples of scribes from the same administrative area writing ${ }^{*} 18$, but the greatest similarity in forms is between H103, H118, and H132, all of whom use Type 1b; these scribes are found respectively in the WW, NEP, and Arsenal, and therefore do not appear be closely related. Since this similarity consists only of using a simplified form, and these differ in both the number of 'horns' (H103 and H118: two; H132: three) and the shape of the 'head' (H103 and H132: round; H118: oval), this may be due merely to similar but independent simplification processes (cf. H117's use of Type $1 \mathrm{~b}$ in minuscule), and is therefore not necessarily significant.

Finally, it is worth considering this issue from the point of view of the scribes as well as of administrative areas: are individual scribes who share a form of one sign more likely also to share forms of others, and is there any correlation of the palaeography of these signs with wider palaeographic groupings? Although Knossian scribes are not divided into palaeographic classes in the same way as Pylian scribes (see p.204), Olivier 1967:27 suggests a possible division based on the use of 'simple' and/or 'complex' forms of $n i$ and sa (e.g. $\Upsilon^{x y}$ vs. $\left.{ }^{x}\right) .{ }^{591}$ The table below shows all the hands who have both examples of $n i$ and/or s $a$ and at least one undeciphered sign (apart from $*_{19}, *_{56}$, and ${ }^{*} 64$ ), classified according to their forms of $n i$ and sa (simple, complex, or both).

\footnotetext{
${ }^{590}$ See p.193, n.582 on the possible link between the WW textile department and J1 via H119; but even if this were certain, it would be a fairly indirect link between these two hands.

${ }^{591}$ The 'intermediate' form, which resembles the simple form but is drawn with three strokes instead of two, appears to be a variation of the simple form rather than a diagnostic feature in its own right, and is therefore included with the simple form here.
} 


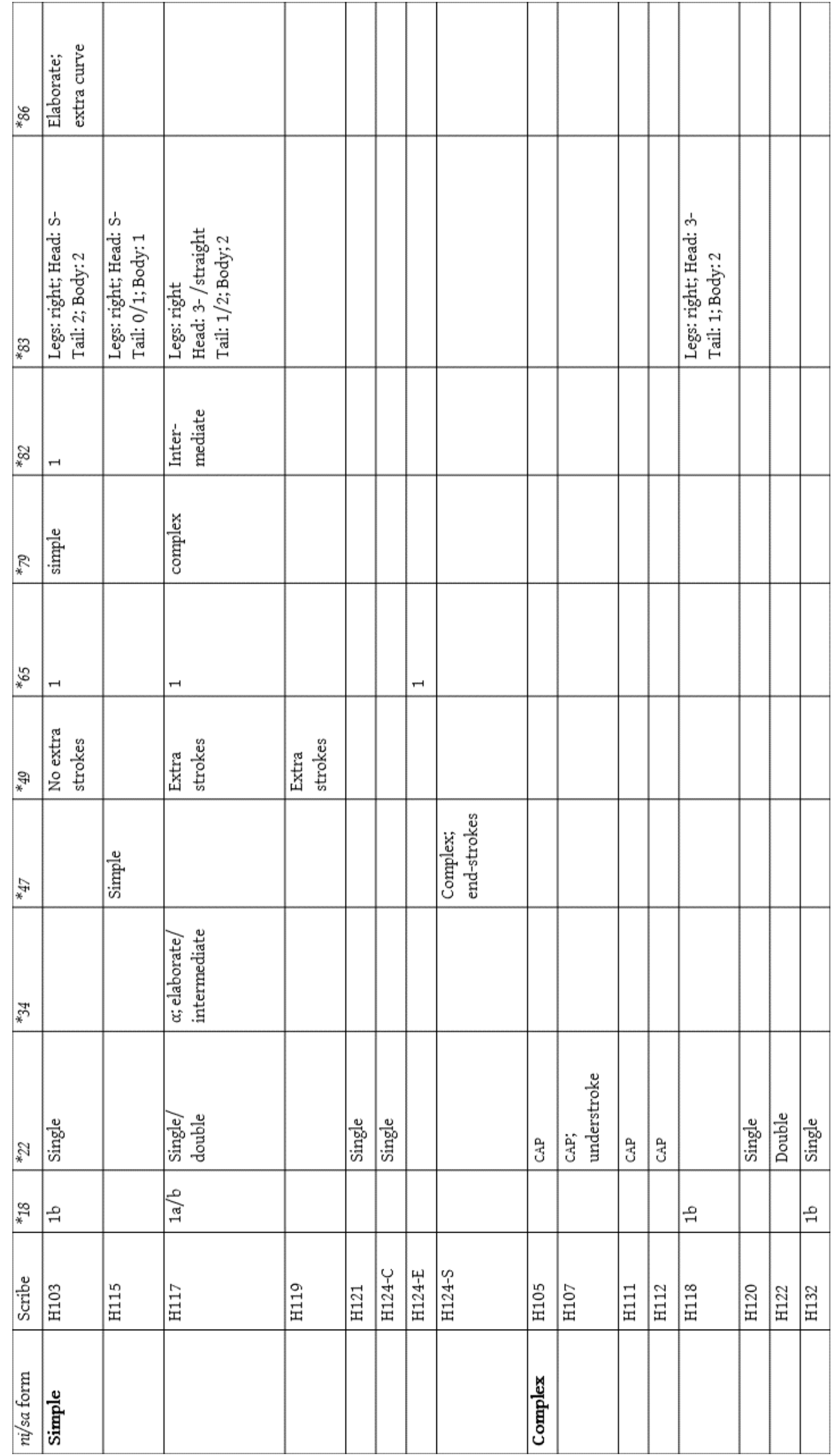




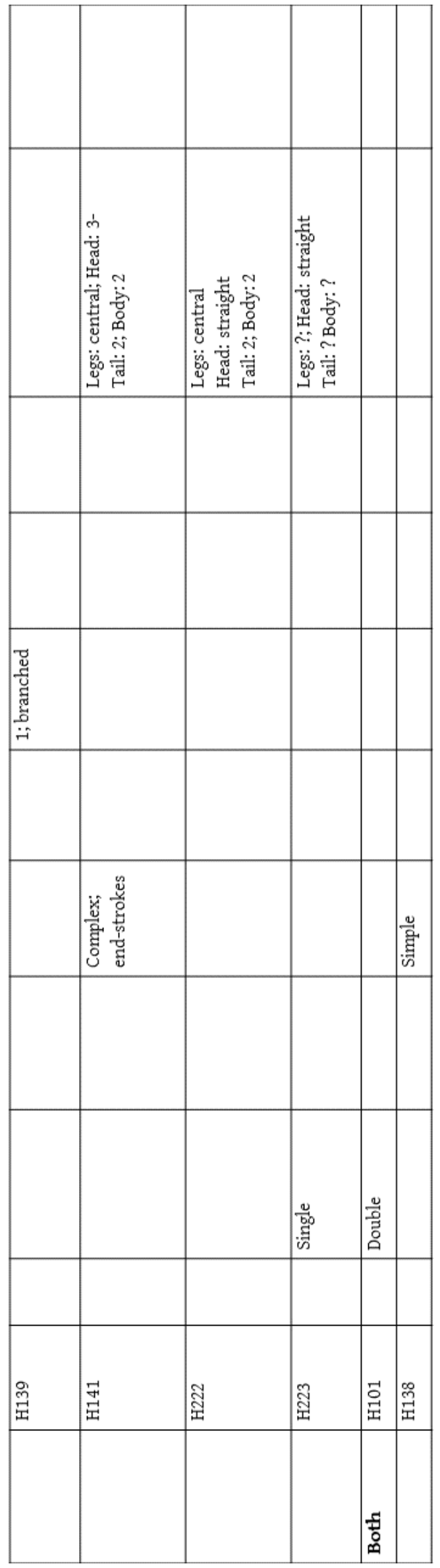


The table shows that there are multiple examples of different forms of an undeciphered sign being attested within a 'class' based on the forms of $n i / s a$ : e.g. the 'simple' ni/sa class contains the simple and complex forms, with and without endstrokes, of *47; both forms of $*^{*} 49,79$, and $* 22$; and various forms of ${ }^{*} 83,{ }^{592}$ while the 'complex' ni/sa class contains all the different forms of $* 22 /$ AAP and a similar variety of forms of *83. Likewise, the same form frequently appears in more than one class (e.g. *18 Type $1 \mathrm{~b}$; single- and double-stalk *22/CAP; both forms of *47; Type $1 * 65$; various features of *83). The few instances where a particular form is only certainly attested in one class (e.g. *22/cAP with an understroke; the 'branched' *65) are as likely to be due to the low number of attestations of this form as to be palaeographically significant. Similarly, a pair of scribes who share a form of one undeciphered sign do not necessarily share forms of others: this is best illustrated by H103 and H117, who share forms of ${ }^{*} 18$ and ${ }^{*} 22$, but differ in $*_{49},{ }^{*} 79, * 82$, and some features of ${ }^{*} 83$.

At Knossos, therefore, there is essentially no correlation between scribes' location(s) and subject(s) of work and their forms of the undeciphered signs: scribes seem to be at least as likely to share a form with an apparently unrelated scribe, and/or to differ in form from a more closely related one, as vice versa. It is particularly noteworthy that in discussing the four signs $\left({ }^{*} 22, *^{*} 4,{ }^{*} 47\right.$, and $\left.{ }^{*} 65\right)$ with multiple attestations from the RCT a deposit which is generally considered to have a strikingly low level of inter-scribal variation, ${ }^{593}$ and probably the most widely-accepted example of palaeography correlating with scribal relationship - in two of the four cases the level of variation in this deposit is equivalent to that seen in the rest of the Knossos tablets $\left({ }^{*} 22\right)$, or even greater $\left({ }^{*} 65\right)$. Overall, the palaeographic situation at Knossos has been shown to be a

\footnotetext{
${ }^{592}$ Plus two examples where the variation within this class is due to variation within H117: ${ }^{*} 18$ and ${ }^{*} 22 /$ CAP. ${ }^{593}$ See, e.g., Driessen 2000:62.
} 
highly complex one, with a relatively wide range of variation in several of these signs which correlates neither with reconstructable administrative relationships nor with other possible means of palaeographic grouping.

\subsubsection{Pylos}

The archaeological situation at Pylos differs significantly in some ways from that of Knossos. The majority of the Pylos tablets have well-documented findspots, and even when tablets have been displaced post-destruction, their contents and/or hand often allow their original location to be identified; the vast majority are also securely assigned to the same chronological layer, that of the palace's final destruction..$^{594}$ Issues relating to uncertainties over findspots or deposits' relative chronology therefore for the most part do not arise at Pylos.

As might be expected in a palace around one-sixth of the size of Knossos ${ }^{595}$ Pylos also has a much smaller number of scribes (26 hands and 17-18 styli, of which Palaima 1988b suggests at least 7 are probably individual hands - cf. Knossos' 41 major and 25 secondary hands, as identified by Olivier 1967); it also displays significant differences in the organisation of its administrative system, ${ }^{596}$ most obviously in the existence of the 'Archives Complex' (Rooms 7-8: henceforth the AC). Although the Knossian RCT and NEP deposits are similar in some ways to the Pylian AC in their status as "non-specialised ensembles ${ }^{597}$ containing the work of a relatively large group of scribes, and the NEP in

\footnotetext{
${ }^{594}$ A small number of tablets have been suggested to be of earlier date (Palaima 1983 and 1988b:111-3, 133, 172; these are attributed to 'H91' and 'Class iv'). Since these tablets contain no examples of undeciphered signs they are excluded from this study. See further p.241.

${ }^{595}$ Olivier 1984:11.

${ }^{596}$ Olivier 1984; Shelmerdine 1988 and 1999.

${ }^{597}$ Shelmerdine 1988:345.
} 
particular shows evidence of systematic filing of documents, ${ }^{598}$ neither is completely comparable to the AC, in which c.80\% of the Pylos tablets were found, and which shows extensive evidence for scribal collaboration and systematic filing and storage of tablets, at least some of which were produced elsewhere in the palace. ${ }^{599}$ Pylos correspondingly shows less evidence of the large-scale 'bureaux' and departments seen at Knossos, although there are still some areas of the palace which may have a similar status to this (see below, pp.206-7). All of these differences mean that the methodology of identifying scribal connections will likewise differ in some respects from that used for Knossos.

Pylos also has a significant palaeographic difference from Knossos in the grouping of its scribal hands and styli into three main 'classes' (Class i, ii, and iii) based on their palaeographic similarity to the 'major' scribe of each class (H1, H21, and H41). ${ }^{600}$ The table below shows the hands and styli with examples of undeciphered signs, arranged by class $(\boldsymbol{V}=$ certain; ? = uncertain; very uncertain examples are excluded).

\footnotetext{
${ }^{598}$ Firth 1996-1997:51-9; cf. p.178, n.546.

${ }^{599}$ Palaima 1988b:179.

${ }^{600}$ PTT II:7-9; Palaima 1988b:30-1. On Class iv, see p.203, n.594. Pylian 'styli’ - groups of tablets which are linked by handwriting and subject-matter, but which cannot confidently be either distinguished as a separate hand or attributed to a known hand - are approximately equivalent to the Knossian 'secondary hands' (p.183, n.559). NB that Olivier 2012b:109-10 challenges the usefulness of the categories of 'class' and 'stylus', which he and L. Godart plan to eliminate in their corpus of the Pylos tablets; since their proposed classifications cannot be properly evaluated until the appearance of this corpus, for the moment I continue to use the scribal classifications given in the published corpora. However, styli will be discussed only in relation to possible links with scribes from different classes, since they cannot certainly be distinguished from scribes within their own class.
} 


\begin{tabular}{|c|c|c|c|c|c|c|c|c|c|c|}
\hline Class & Scribe & $*_{19}$ & $* 22 /$ CAP $^{601}$ & $*_{34}$ & $* 63$ & $*_{64}$ & *65/FAR & $* 79$ & $* 82$ & ${ }^{*} 83^{602}$ \\
\hline \multirow[t]{7}{*}{$\mathrm{i}$} & $\mathrm{H} 1$ & & $\checkmark$ & $\checkmark$ & $\checkmark$ & $\checkmark$ & $\checkmark$ & $\checkmark$ & $\checkmark$ & $\checkmark$ \\
\hline & $\mathrm{H} 2$ & $?$ & & $\sqrt{ }$ & & $\checkmark$ & $\checkmark$ & & $\sqrt{ }$ & \\
\hline & $\mathrm{H} 3$ & $\checkmark$ & & & & & & & & \\
\hline & H6 & & $?$ & & & & $\checkmark$ & & $\sqrt{ }$ & \\
\hline & S155 & & $?$ & & & & & & & \\
\hline & S1331 & & $\checkmark$ & & & & & & & \\
\hline & $--^{603}$ & & $?$ & & & & & & $\checkmark$ & \\
\hline \multirow[t]{7}{*}{ ii } & $\mathrm{H} 21$ & & $\checkmark$ & $\checkmark$ & & $\checkmark$ & $\checkmark$ & & $\checkmark$ & \\
\hline & $\mathrm{H} 22$ & & & & & & $\checkmark$ & & & \\
\hline & $\mathrm{H} 23$ & & & & & $\checkmark$ & & & & \\
\hline & $\mathrm{H} 24$ & & & & & & $\checkmark$ & & & \\
\hline & $\mathrm{H} 25$ & & & & & & & & $\checkmark$ & \\
\hline & $\mathrm{H} 31 / 27^{604}$ & & $\checkmark$ & & & $\checkmark$ & & & & \\
\hline & - & & $\checkmark$ & $\checkmark$ & & & $\checkmark$ & $?$ & $\checkmark$ & $?$ \\
\hline \multirow[t]{6}{*}{ iii } & $\mathrm{H} 41$ & & & $\checkmark$ & $\checkmark$ & & $\checkmark$ & $\checkmark$ & & $\checkmark$ \\
\hline & $\mathrm{H} 42$ & & $\checkmark$ & & & & $?$ & $\checkmark$ & & \\
\hline & $\mathrm{H} 44$ & & & & & & & & $\checkmark$ & \\
\hline & S324 & & & & & $\checkmark$ & & & & \\
\hline & S1272 & & & & & & & & $\checkmark$ & \\
\hline & - & & $\checkmark$ & $\checkmark$ & & $\checkmark$ & & & & \\
\hline
\end{tabular}

In identifying administrative connections between these hands and styli, tablet findspots outside of the AC may be used in a similar way to Knossos; the most important of these areas for the purposes of this section are the North-Eastern Building (NEB: Rooms 98-99)

${ }^{601}$ Attested only as CAP at this site.

${ }^{602}$ Signs omitted: *18, *47, *49 (not attested at Pylos), *56 (no significant palaeographic variation), ${ }^{*} 86$ (attested only in texts attributed to $\mathrm{H} 1$ ).

${ }^{603}$ I.e. examples attributed to a class but not to a specific hand or stylus. Some of these attributions may be more secure than others, depending on factors such as the number of different signs found on the tablet in question; this will be discussed where relevant below.

${ }^{604}$ This hand is designated as H31 in Palaima 1988b, but H27 in PoN IV; since Cii hands in the 20's are those which are palaeographically closer to H21, and those in the 30's are more similar in some respects to Ciii (Palaima 2011:55, n.36), the editors of PoN IV presumably consider this hand to be palaeographically closer to $\mathrm{H} 21$ than previously thought. This hand will here be referred to as H31/27 in order to enable references to works using either designation. 
and the various rooms dealing with oil storage and distribution (Rooms 23, 24, 32, and $38) .^{605}$

\section{Imageremoved for copyright reasons}

\section{Archives Complex - Oil storage areas - North Eastern Building ${ }^{606}$}

The NEB has been convincingly argued by Bendall 2003 to be a 'clearinghouse' with administrative functions similar to those of a Knossian department, including at least the short-term storage of documents relating to a wide range of subjects. ${ }^{607}$ of the hands above, H21, H31/27, S1331-Ci, and S1272-Ciii are found in this location (plus the Cii hand of $\mathrm{Cn} 1286$, with one example of $\left.{ }^{*} 22 / \mathrm{CAP}\right)$. Of these, most are attested as working

\footnotetext{
${ }^{605}$ Other administrative areas identified in the palace, such as the probable textile workshop/storeroom above rooms 5-6 (Jasink 1990-1991:228-30), the Wine Magazine (Room 105: Palaima 1988b:159-60), and the South-West Building (Shelmerdine 1998-1999), will not be discussed here since they do not form links between any of the scribes listed above.

${ }^{606}$ Map adapted from Palaima-Shelmerdine 1984, fig.2.

${ }^{607}$ Note that the NEB and the AC are closely linked by the presence of some scribes, and even one tablet series (Sa-, H26), in both locations.
} 
specifically on livestock tablets (H21: Cc-series, CAP and ovIs; cf. this hand's Cn-series from the AC: CAP, OVIS, and SUS. H31/27: Cn 1287, CAP; Ub 1315, animal hides. S1331-Ci: sealings listing $\mathrm{CAP}^{\mathrm{m}}$ ), but S1272-Ciii has only Ac-series personnel tablets. The administrative system relating to perfumed oil production has been reconstructed by Shelmerdine $1985,{ }^{608}$ who shows that $\mathrm{H} 2$ provides a link between all of the various oilrelated deposits and the AC: ${ }^{.09} \mathrm{H} 41$ (found in Room 38) therefore has an administrative connection to $\mathrm{H} 2 .{ }^{610}$

For the tablets found in the $\mathrm{AC},{ }^{611}$ however, location is not a particularly useful indicator of possible administrative relationships, given the large number of both tablets and scribal hands (at least 19) found in this location, as well as the fact that many of the AC tablets may well have originally been produced elsewhere in the palace. ${ }^{612}$ In some cases comparison of exact findspots within the AC may help to suggest connections between groups of tablets (Pluta 1996-1997): for instance, H2's Ta-series and H24's Un 718 were

\footnotetext{
${ }^{608}$ Kyriakidis 2011:215-7 argues (against Shelmerdine's reconstruction) that the majority of the scribes found in these areas are actually specialised in other aspects of the administration, which occasionally leads to their production of oil-related tablets. However, this appears to be based more on a desire to prove a high degree of subject specialisation for almost all Pylian scribes than on the actual evidence of the tablets. For the purposes of reconstructing administrative connections, all of these scribes can be deemed to be concerned with this industry and thus broadly connected to each other in some respect. ${ }^{609}$ On this hand, see also Kyriakidis 2011.

${ }^{610} \mathrm{Fr} 1223$ (Room 23) is attributed to H44 by PTT and Palaima 1988b, which would give this hand a similar connection to H2; however, PoN IV and Olivier 2012b:111 both suppress this attribution. Due to the uncertainty over this attribution, as well as over whether H44's only other tablet, Tn 316, is in fact the product of two hands (Godart 2009), this possible link will be excluded from discussion here. Note that even if Godart's argument that ' $\mathrm{H} 44$ ' is actually two hands is correct, both of the examples of * 82 on Tn 316 would be by the same hand (Godart's H44b); I therefore refrain from taking a position on this question, and continue to refer to this hand as $\mathrm{H} 44$ for the sake of convenience.

${ }^{611} \mathrm{NB}$ that in some cases, tablets found in other areas have been included as coming from the AC since their hand and series attributions and/or joins to AC fragments suggest that this was their original location: see Palaima 1988b:162-6; Shelmerdine 1998-1999.

${ }^{612}$ Evidence for the transfer of documents to the AC is provided by H26's Sa-series, which is split between the AC and NEB: this seems more likely to be due to their production in the NEB and subsequent transfer to the AC (Palaima 1988b:179; Bendall 2003:220) than to the task of recording wheels being divided between the two locations (Jasink 1990-1991:226).
} 
found together in the centre of Room 7, perhaps fallen from a worktable, which might imply that they had not yet been filed and so could refer to the same feasting event (Kyriakidis 2011:128). In most cases, however, it is necessary to look at other aspects of the tablets in order to establish possible links between scribes.

Working on the same broad area of the administration does not necessarily indicate a particularly close connection between AC scribes unless this is combined with other kinds of evidence (e.g. location); however, a more narrowly-defined shared subjectmatter may be of greater use in this respect. For instance, H1's Aa-series tablets, H21's Ab-series, and H23's Ad-series all deal with the same groups of people (though none is obviously based directly on any of the others) $;^{613}$ cf. the possibility that H2's Ta-series and H24's Un 718 refer to the same event. Both of these imply a potential administrative connection between the scribes concerned; however, it cannot be shown that these scribes were directly collaborating, rather than working independently on different aspects of the same subject. A much clearer direct link between scribes is shown in cases where one scribe has used another's tablets in order to compile their own: H41's Eb- and Eo-series tablets are preliminary records of the same landholdings as H1's longer Ep- and En-series records; ${ }^{614} \mathrm{H} 2$ 's Jn-series tablets at least partly make use of those written by $\mathrm{H} 21 ;{ }^{615} \mathrm{H} 1$ and $\mathrm{H} 21$ have each written a tablet recording an allocation of GRA 5 to ke-sa-da-ra (Fg 368 and 868), and H1's Fg 253 may be the totalling tablet based on both scribes' records (Palaima 1988b:84). A similarly direct link is shown by interventions by one scribe on another's tablet to either correct or add to existing information: H1 adds extra information to H21's Cn 595, 599, and 655, and has apparently erased and rewritten

\footnotetext{
${ }^{613}$ Palaima 1988b:51-2, 88-9.

${ }^{614}$ Palaima 1988b:102.

${ }^{615}$ Smith 1992-1993:203.
} 
Ed 411.2 (of which .1 and probably [[.2]] are by H41). ${ }^{616}$ Strictly speaking, examples such as these demonstrate only a unidirectional interaction by the correcting/editing/ compiling scribe with the other scribe's documents; however, the fact that the scribes performing these tasks are generally $\mathrm{H} 1$ and $\mathrm{H} 2$ - who appear to be not only the two most prolific scribes, but also the two with the greatest range of responsibilities and therefore, presumably, the highest status ${ }^{617}$ - implies that this may be a hierarchical relationship, with the superior scribe not only using and checking a subordinate's work but potentially also playing a supervisory role.

The palmprints found on tablets - left during the process of making rather than writing on them - may provide another form of link between scribes. ${ }^{618}$ For instance, prints of the same tablet-maker ('Energetikos') are found on tablets by H21, H41, and H43; however, it is not possible to infer from the distribution of these and other prints whether they were left by the scribes themselves, other people acting as tablet-makers, or a combination of the two. Although this demonstrates some form of connection between $\mathrm{H} 21$ and H41 (and H43, but this hand is not discussed here), the nature of this remains unclear: it could be a close working relationship based on, e.g., some (now unreconstructable) subject-based link between their tablets (Palaima 1985:105-6), or it could simply be that they happened to be working on these tablets in the same location (the AC?) at the same time. ${ }^{619}$

\footnotetext{
${ }^{616}$ Palaima 1988b:102.

${ }^{617}$ Kyriakidis 2011:136-7.

${ }^{618}$ See Sjöquist-Åström 1985, including the Appendix by T.G. Palaima.

${ }^{619}$ Further possible methods of identifying connections between scribes (e.g. the 'borrowing' of tablets made for/by another scribe; the writing of filing labels for another scribe's tablet series; and instances of different scribes writing on sealings impressed by the same seal) will not be discussed here as there are no examples concerning the scribes listed above.
} 
Finally, the relationship between $\mathrm{H} 1$ and $\mathrm{H} 2$ should be briefly discussed. The two have no direct or indirect administrative links of the types reconstructed here, but have been shown by Kyriakidis 2011 to hold very similar positions relating to the overall management of administration dealing with, respectively, extra- and intra-palatial matters (cf. p.209 on these scribes' large number of tablets, wide-ranging responsibilities, and oversight of other scribes' work); they therefore present a complementary relationship, rather than one necessarily involving any direct administrative interactions.

\section{$\underline{\text { Summary of connections between scribes }}$}

< > direct hierarchical relationship (superior > subordinate)

- non-hierarchical close/direct connection (e.g. same subject in same location)

$\sim$ less direct or less certain connection (e.g. tablets dealing with same narrow subjectarea but not directly related; same location but not same subject; palmprints)

At least one direct relationship:

$\mathrm{H} 1>\mathrm{H} 21, \mathrm{H} 41 ; \sim \mathrm{H} 23$

$\mathrm{H} 2>\mathrm{H} 21 ;-\mathrm{H} 41 ; \sim \mathrm{H} 24$

H21 < H1, H2; - H31/27, S1331-Ci; H23, H41, S1272-Ciii

H31/27 - H21, S1331-Ci; S1272-Ciii

$\mathrm{H} 41<\mathrm{H} 1 ;-\mathrm{H} 2 ; \sim \mathrm{H} 21$

S1331-Ci - H21, H31/27; S1272-Ciii

Only indirect/less certain relationships:

H23 H1, H21

$\mathrm{H} 24 \sim \mathrm{H} 2$ 
S1272-Ciii H21, H31/27, S1331-Ci

No reconstructable links: H3, ${ }^{620} \mathrm{H} 6,{ }^{621} \mathrm{H} 22,{ }^{622} \mathrm{H} 25,{ }^{623} \mathrm{H} 42,{ }^{624} \mathrm{H} 44,{ }^{625} \mathrm{~S} 155-\mathrm{Ci},{ }^{626} \mathrm{~S} 324-\mathrm{Ciii}^{627}$

Palaeography of the undeciphered signs at Pylos

$\underline{*_{19}}$ 主 (pp.77-9)

All four probable examples of this sign are in Ci hands; ${ }^{628}$ they have a similar basic form, with a round head, a separate horizontal beneath, and a body with slanting sides, but the condition of Jn 725.3 makes it impossible to say whether $\mathrm{H} 2$ also had the middle vertical seen in $\mathrm{H} 3 / \mathrm{H} 3$ ? $^{629}$

\section{Images removed for copyright reasons}
An $427.3(\mathrm{H} 3)$
Vn 10.2.5 (H3?)
Jn 725.3 (H2)

\footnotetext{
${ }^{620}$ Kyriakidis 1996-1997:225 suggests a connection between H3 and H6, describing them as hands 'which overlap but whose subject is not clear at all'. As $\mathrm{H} 3$ has written tablets dealing with personnel, rations, and wood for chariots, while H6's tablets record offerings to Poseidon and transactions involving alum, there is no basis to posit such a connection.

${ }^{621}$ See $n .620$ above.

${ }^{622} \mathrm{Ae}$ - and An-series (AC); no obvious connections to other personnel tablets.

${ }^{623} \mathrm{Vn} 20$ (AC; wine distributions); no obvious parallels.

${ }^{624}$ Personnel tablets (Ae-, An-), various mixed livestock/food commodity tablets (Fa-, Ua-, Un-), and ivory (Va 482: all AC), but with no obvious direct connection to any other scribe working on broadly similar subjects.

${ }^{625}$ See p.207, n.610.

${ }^{626}$ The only reasonably probable link involving this stylus is with $\mathrm{H} 1$, since both not only have AC Cn-series tablets, but also share a fairly similar tablet format (Palaima 1988b:116). As the two are from the same class, this could equally form an argument why S155 should perhaps be identified with $\mathrm{H} 1$, so this possible link will be disregarded (cf. p.204, n.600).

${ }^{627} \mathrm{Fn} 50$ and 324 (AC); these do not appear to correspond to H2 and H45's Fn-series tablets (Palaima 1988b:111).

${ }^{628}$ If $\mathrm{Vn} 10$ is not by $\mathrm{H} 3$, this would provide an example of two hands sharing a remarkably similar form, and yet differing in the details of that form, but little more could be said other than that this would indicate the potential range of variation even amongst palaeographically similar hands.

${ }^{629}$ The more doubtful example on Wr 1374.Y (Ciii) is excluded here.

${ }^{630}$ Photos: author (NAM).
} 


\section{$\underline{* 22 / \text { CAP }} \bigwedge^{\prime}(p p .86-7)$}

At Pylos, the presence or absence of a vertical stroke at the end of the head of CAP is the only diagnostic form of variation seen in CAP. H1 consistently has this feature (which is also seen in S1331-Ci, perhaps H6 and S155-Ci, and all the examples attributed to unknown Ci, Cii, and Ciii hands), ${ }^{631}$ while the identified Cii and Ciii hands (H21, H31/27, and H42) consistently do not. In hands which do not have the vertical stroke, H21 and H31/27 (both Cii) - vary in a similar way between 'wavy' and 'right-angled' heads (the latter not being found at other sites); it is not clear whether H42 (Ciii) would do the same if more examples were preserved. A significant number of examples of CAP are in hands associated with the NEB (H21 and H31/27; Ci hands of Wr 1325 and 1334; Cii hand of Cn 1286).

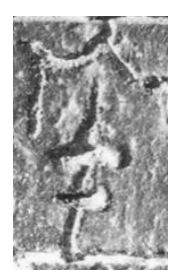

Cn $643.5(\mathrm{H} 1)$

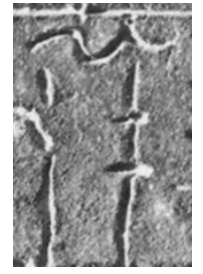

Cn $599.2(\mathrm{H} 21)$

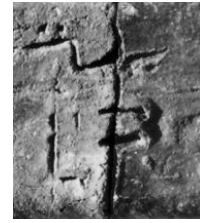

Cc $660(\mathrm{H} 21)^{632}$

*34 $\underset{\phi}{\phi}$ (pp.92-3)

This sign's orientation is the only significant variant feature at Pylos: $\mathrm{Ci}(\mathrm{H} 1, \mathrm{H} 2)$ and $\mathrm{H} 21$ use ${ }^{*} 34 \alpha$, while H41 and the unidentified Cii and Ciii hands use ${ }^{*} 34 \beta .{ }^{633}$ The only example from outside the AC is Wr 1374. $\gamma$ (Ciii), perhaps originating from Room 6 (Shelmerdine 1998-1999:329).

\footnotetext{
${ }^{631}$ Uncertain example in Ci hand of $\mathrm{Cn}$ 440.5; certain examples in Cii hand of $\mathrm{Cn} 1286$ and Ciii hand of $\mathrm{Cn}$ 418. Neither of the latter can be regarded as completely secure due to the low number of different signs certainly attested on the tablets.

${ }^{632}$ Photos: MER.

${ }^{633}$ The attribution of the Cii hands is relatively secure: Va 15 has 20 different signs and Vn 4632 different signs. The Ciii example, however, is much less secure as it appears on a sealing (Wr 1374) with only four different signs.
} 
*63 㭌 (pp.124-6)

This sign is found only in H1, who uses the elaborate form, and H41, who uses the intermediate form (midway between the simple and elaborate ones).

\section{Images removed for copyright reasons}

En 74.14 (H1) Eo $247.5(\mathrm{H} 41)^{634}$

\section{${ }_{64}$ 园 $($ pp.128-9)}

This sign varies only in the presence or absence of small strokes at the ends of each vertical: $\mathrm{Ci}(\mathrm{H} 1, \mathrm{H} 2)$ and some Cii hands (H21, H31/27) have these strokes, while H23, S324-Ciii, and the Ciii hand of Vn 34 do not. ${ }^{635}$

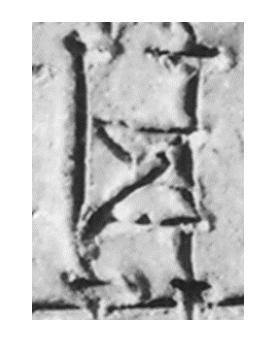

Jn $832.11(\mathrm{H} 2)$

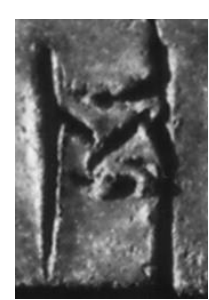

Vn 34.2 (Ciii) ${ }^{636}$

\section{*65/FAR W}

The more elaborate 'Group 1' forms of this sign are used by Ci (H1, H2, H6; the last of these has a unique mainland form, closer to the usual Knossian form), the Cii hand of Vn

\footnotetext{
${ }^{634}$ Photos: author (NAM).

${ }^{635}$ These attributions are both relatively secure: S324-Ciii consists of $\mathrm{Fn} 50$ (42 different signs) and $\mathrm{Fn}$ 324+1454 (49 different signs), while Vn 34 has 31 different signs.

${ }^{636}$ Photos: MER.
} 
46, ${ }^{637}$ and perhaps $\mathrm{H} 42{ }^{638}$ the simpler 'Group 2' forms are used by other Cii and Ciii hands (H21, H24, and the Cii hand of Vn 879; H41), ${ }^{639}$ and H22 has an intermediate form.

\section{Images removed for copyright reasons}

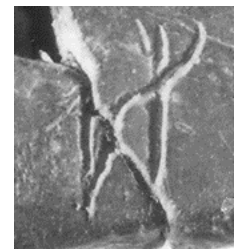

Jn $725.8(\mathrm{H} 2)$

Un $853.5(\mathrm{H} 6)$

Eo 276.8 (H41)

Ae $344(\mathrm{H} 22)^{640}$

*79 :

Of the four probable examples of this sign, three have the more elaborate form with small strokes around the outside (H1, H41, H42); the simple form probably appears on Va 404 (Cii). ${ }^{641}$

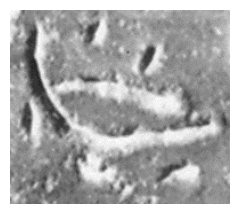

Va $482(\mathrm{H} 42)$

\section{Imageremoved for copyright reasons}

$\mathrm{Va} 404^{642}$

$\left.{ }^{*} 82\right\}_{2}^{2}($ pp.162-4)

Type 1 forms (with a '3'-shaped central part) are used by $\mathrm{Ci}\left(\mathrm{H} 1, \mathrm{H} 2,{ }^{643} \mathrm{H} 6\right.$, and the $\mathrm{Ci}$ hand of Jo 438) ${ }^{644}$ and by H25 (who has a uniquely deeply-curved form); Type 2 (with a

\footnotetext{
${ }^{637}$ As this tablet has 32 different signs its class attribution is relatively secure.

${ }^{638}$ The traces of erasure on Un 47.4, read [[FAR]], are more compatible with a Group 1 form of this sign than Group 2.

${ }^{639}$ As this tablet has 19 different signs its class attribution is reasonably but not entirely secure.

${ }^{640}$ Photos: author (NAM) except 344 (MER).

${ }^{641}$ As this tablet has only 3 certainly-attested syllabograms, its attribution to Cii is highly insecure.

${ }^{642}$ Photos: MER; author (NAM).

${ }^{643}$ This hand also has one simplified form closer to Type 2 on Ma 225.1.

${ }^{644}$ As this tablet has 48 different signs its attribution seems secure. NB: if H3's uncertain example on An 424.1 is * 82 , this would also have to be a Type 1 form; however due to the uncertainty of this reading this is excluded from the discussion here.
} 
curved central part) is used by other Cii hands (H21 and the Cii hand of Vn 19) ${ }^{645}$ and Ciii (H44 and S1272-Ciii).

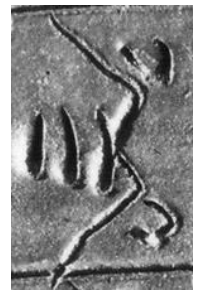

Un $6.3(\mathrm{H} 6)$

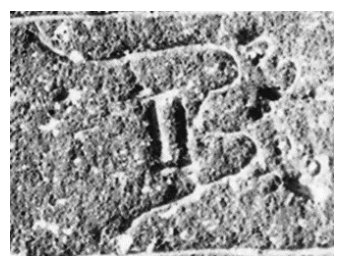

Vn $20.3(\mathrm{H} 25)$

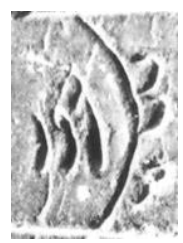

Vn 19.1 (Cii)

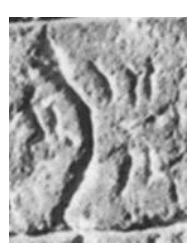

$\operatorname{Tn} 316.5(\mathrm{H} 44)^{646}$

The only Pylian examples of * 82 which do not have three vertical strokes to the left are H44's (Tn 316.4 may have two or three; .5 certainly has two) and H25's, which has a rectangle. The elements to the right of the sign may vary even within a single hand (e.g. H1 and H2 may have either one or two curves at top and bottom; H6's two examples on Un 6.3-4 differ from each other in this respect); the only scribe with more than one example of $* 2$ who is consistent in this respect is $\mathrm{H} 44$, whose two examples both have an unparalleled three curves (one after the other) at top and bottom. That H21, H25, the Cii hand of Vn 19.1, and S1272-Ciii have two curves to the right of *82 and the Ci hand of Jo 438.26 only one is therefore not necessarily significant. Two of the hands using this sign (H21 and S1272-Ciii) are associated with the NEB.

*83 玣

This sign is only certainly found in $\mathrm{H} 1$ and $\mathrm{H} 41$ at Pylos (the two other possible examples are both extremely uncertain and attributed only to Cii [Va 15.1] and to S622, tentatively assigned to H13?; hence these are excluded here). H1 uses a symmetrical form of the sign, with 'legs' of equal length on both sides; H41's form appears to be a slightly simplified version of this, shorter on the right-hand side, and often with only one 'leg'

\footnotetext{
${ }^{645}$ As this tablet has only 13 different signs, its class attribution is not particularly secure. ${ }^{646}$ Photos: MER.
} 
instead of two. The form of *83's head also varies in both hands from '3'-shaped/two small curves to a single stroke.

\title{
Images removed for copyright reasons
}

\author{
En 74.1 $(\mathrm{H} 1) \quad$ Eo $276.2(\mathrm{H} 41)^{647}$
}

\section{Discussion}

\section{Correspondence with palaeographic classes}

Since the Pylos palaeographic classes are established on the basis of similarities of handwriting to the 'major scribe' of each class (Ci: H1; Cii: H21; Ciii: H41), and it has generally been assumed (following Palaima 1988b:187) that these classes represent groups of scribes who underwent similar training, perhaps even taught by the "major scribes', it would be logical to expect that in cases where different variant forms of a sign exist, hands of the same class would share the same variant, while hands of different classes might differ.

Support for this assumption from this case-study appears, however, to be limited. *63 and ${ }^{*} 83$ show different forms in hands of different classes, with the Ciii form being slightly simplified compared to the Ci form in each case; however, these cases both involve only two scribes, $\mathrm{H} 1$ and $\mathrm{H} 41 .{ }^{*} 19$ shows up to 3 hands of the same class (Ci: H2, H3, H3?) probably using similar forms, but there are no examples in other classes for comparison, and even within these hands some variation can be seen.

\footnotetext{
${ }^{647}$ Photos: author (NAM).
} 
In several cases, a pattern emerges whereby $\mathrm{Ci}$ and $\mathrm{Ciii}$ hands each have a different variant form, but Cii hands may select either (though each individual hand will generally use only one). ${ }^{*} 34 \alpha$ is used by $\mathrm{Ci}$ and $\mathrm{H} 21,{ }^{*} 34 \beta$ by Ciii and two unidentified (but reasonably securely attributed) Cii hands; similarly, Ci hands write *64 with the extra strokes and Ciii hands without these, but in Cii both forms are found (H21 and H31/27: with these strokes; H23: without). ${ }^{*} 65$ and ${ }^{*} 82$ show a similar, but slightly more complex, pattern: Ci hands mostly use the Group 1 form of *65 and Ciii Group 2, while Cii also mostly uses Group 2 but has a single Group 1 example in the (relatively securely attributed) Cii hand of Vn 46. The picture here is complicated by the existence of significant variation within Group 1 (H6's unique mainland form), the intermediate form also found in Cii (H22), and the possible Group 1 example in H42, which, if correctly read, would mean that Ciii also used both forms. Similarly, in the case of *82, Ci generally uses Type 1 (H2's single exception appears to be a one-off simplification), and Ciii uses Type 2, while Cii has both (Type 1: H25; 2: H21, hand of Vn 19); however, unique forms of the elements to the left and right of the sign are found in one Cii hand (H25) and one Ciii hand (H44).

As stated above (p.205, n.604), Cii is divided into two groups, with hands numbered in the 20's being closer to H21 (and presumably also to Ci), and those in the 30's resembling Ciii in some respects. However, the distribution of variant forms amongst Cii hands does not always correspond to this division: for instance, $\mathrm{H} 21$ and $\mathrm{H} 31 / 27$ share the $\mathrm{Ci}$ form of ${ }^{6} 64$, but H23 shares the Ciii form; H21 and H24 use Group 2 forms of *65, as generally used by Ciii, not the Group 1 forms used by Ci and one other (unidentified) Cii hand; H25 shares a Type 1 form of * 82 with $\mathrm{Ci}$, but $\mathrm{H} 21$ shares Type 2 with Ciii. There is thus no overall consistency within a single palaeographic class, within the subdivisions of Cii, or even 
within a single hand, in terms of their palaeographic affiliation in the forms of these signs - H21, in particular, contains features resembling both $\mathrm{Ci}$ and Ciii.

In addition, even this pattern may not hold for all cases where a form is shared across classes. Sign *79 appears in the same form in Ci and Ciii, but (if the rather insecure attribution of Va 404 is accepted) a simplified form in Cii; of the variant forms of $*_{22}$, the one with a vertical stroke may be found in all classes (though again, the attributions to Cii and Ciii are fairly insecure), and both Cii and Ciii certainly have the form without it. The two hands which share the most similar range of variation in the latter form, H21 and $\mathrm{H} 31 / 27$, are both Cii, but there are not enough examples to say that this is certainly not also the case in Ciii.

The palaeographic situation at Pylos is thus more complex than the simple division into classes suggests: relatively clear divisions of variant forms between classes can be seen only in cases with very few examples, suggesting that the more complex situations seen in all other cases - in which variant forms may be shared between classes and/or coexist with other variants within a single class, in various different patterns - may be more representative. Of course, these classes must be founded on the basis of overall tendencies towards similarity between hands, rather than complete identity of individual sign-forms; nonetheless, the results of this case-study suggest that rather than viewing these classes as rigidly-defined entities, each created exclusively through training by a single individual, they should be seen as relatively fluid categorisations, potentially created by a much more complex system of training and/or scribal interactions. Incidentally, this also shows the attribution of tablets or sealings with relatively few signs to a particular class to be considerably more problematic than the presentation of such attributions in the corpora currently suggests. A complete 
palaeographic study of Pylian LB would, however, enable both of these issues to be investigated more fully.

\section{Correspondence with administrative connections}

The question to be addressed here is whether examples can be found where, once palaeographic class has been eliminated as a factor, administrative links appear to have affected scribes' use of forms of these signs.

One of the closest relevant administrative links is that between $\mathrm{H} 1$ and $\mathrm{H} 41$, whose examples of *63 and *83 appear in the same MNs on closely-linked tablets (H1's En-series being based on H41's Eo-series), ${ }^{648}$ but who differ in the forms of not only these two signs but also of $*_{34}$ and $* 65$ (although they share a form of *79, this is also seen in the unrelated H42). H41's connection to H2 via the oil industry likewise does not correlate with their palaeography, as the two differ in the forms of both the undeciphered signs which they share examples of $\left(*_{34}\right.$ and $\left.*^{*} 65\right)$. Compare also the fact that the two extant tablets recording e-wi-su-*79-ko (by H41 and perhaps a Cii hand) have different forms of *79. All of these instances could be attributed to class divisions; the same goes for the close similarity of $\mathrm{H} 1$ and $\mathrm{H} 2$ in every case where they share examples of a given sign $\left({ }^{*} 34,{ }^{*} 64\right.$, $*_{65}$, 82). However, of the signs which have more than one variant attested in Cii, H21 (who is closely linked to both $\mathrm{H} 1$ and $\mathrm{H} 2$ ) shares the $\mathrm{Ci}$ forms of *34 and *64, but differs in ${ }^{*} 65,{ }^{*} 82$, and CAP (though the attribution of both variants of the latter to Cii is less secure than that of the others); this hand's close connection to the two main Ci hands is

\footnotetext{
${ }^{648}$ Although there are many quite considerable differences between corresponding entries in this series (in, e.g., phrasing, formatting, the inclusion/omission of information, and spelling), there are some instances where deviations from H1's usual formula appear to be due to their copying H41's entries (Salgarella 2015 [unpublished]).
} 
therefore not palaeographically significant. There is likewise no apparent correlation between other hands' administrative links and their use of forms differing from their class's usual form: H23, for instance, uses the same form of *64 as S324-Ciii, and H25 the same form of ${ }^{*} 82$ as $\mathrm{Ci}$ hands, but neither has any known links to hands of these classes; the same goes for H42's possible use of the form of *65/FAR seen in Ci and one Cii hand.

The range of examples of undeciphered signs in the NEB provides an opportunity to examine the relationship between location and palaeography. $\mathrm{H} 21$ and H31/27 are closely linked via subject-matter at this location, and share forms of cAP and *64 (cf. the use of the other form of *64 by H23, also Cii but with only indirect links to H21 via their similar personnel tablets). However, $\mathrm{H} 21$ and $\mathrm{H} 31 / 27$ are equally closely linked to the other NEB hands who have also written CAP (attributed, albeit not particularly securely, to S1331-Ci and Cii), ${ }^{649}$ using the other form. H21 and S1272-Ciii share a form of *82; however, this does not seem particularly likely to be related to their location, since they work on different subjects (livestock; personnel) and since they also share this form with H44, who has no known connection to either of these hands or to the NEB.

It is perhaps noteworthy that, when unique forms of signs are found, it is usually in hands with no (certain) administrative links: *65 has unique forms in $\mathrm{H} 6$ and $\mathrm{H} 22,{ }^{*} 79$ in the (possibly Cii) hand of Va 404, and * 82 in $\mathrm{H} 25$ and H44. ${ }^{650}$ It has been suggested that the use of unusual sign-forms, particularly those seen on sealings, may reflect a physically and/or administratively 'peripheral' status (Palaima 2000). However, all of the tablets with unusual forms of undeciphered signs were found in the AC; although they may, of course, have originally been written elsewhere, the fact that they were stored in the AC

\footnotetext{
${ }^{649}$ Note that as the two Ci examples are on sealings, they may have been written and brought from elsewhere; however, the Cii example is on a tablet.

650 'Unique' in this context refers to attestations at Pylos; some of these forms have parallels at other sites.
} 
suggests their contents were not merely of peripheral concern - particularly in the cases of $\mathrm{H} 6$ and H44, who have written what are clearly highly important documents relating to religious offerings. The fact that none of these scribes have written more than a few documents (if this is not due simply to chances of survival) could perhaps suggest a different kind of 'peripheral' status - not that they were working on topics of lesser importance, but that the nature of their work only required them to produce documents relatively infrequently. However, pending further investigation of Pylian palaeography, it seems better to see these unusual forms simply as reminders that the extent of variation is greater than is often assumed. As at Knossos, the overall palaeographic situation at Pylos is highly complex, with scribes' use of variant forms of these signs showing no straightforward correlation with either their administrative relationships or, more surprisingly, with their class membership.

\subsubsection{Thebes}

The relationship between the various excavated Mycenaean structures at Thebes, and hence between the various deposits of LB tablets found in them, is still poorly understood. ${ }^{651}$ There are no examples of identified scribes being found in more than one deposit, and few other possible inter-deposit administrative links; in addition, the chronological relationship between different deposits is highly unclear (not least because the pottery from most of these locations remains largely unpublished; see pp.242-3), and even identifying structures as 'palatial' poses difficulties. ${ }^{652}$ Discussion in this section will therefore largely focus on palaeographic variation within the individual deposits which have examples of undeciphered signs, as indicated on the map below. ${ }^{653}$

\footnotetext{
${ }^{651}$ For an overview of the archaeology of LBA Thebes, see Dakouri-Hild 2010:696ff.

${ }^{652}$ See Dakouri-Hild 2010:698-9. On the relationship between the two structures generally considered to be 'palatial' in the architectural sense - the 'House of Kadmos' and the TC/RP building - see, e.g., Dakouri-Hild 2001.

${ }^{653}$ Adapted from $T F C^{3}: 10$, plan 1.
} 


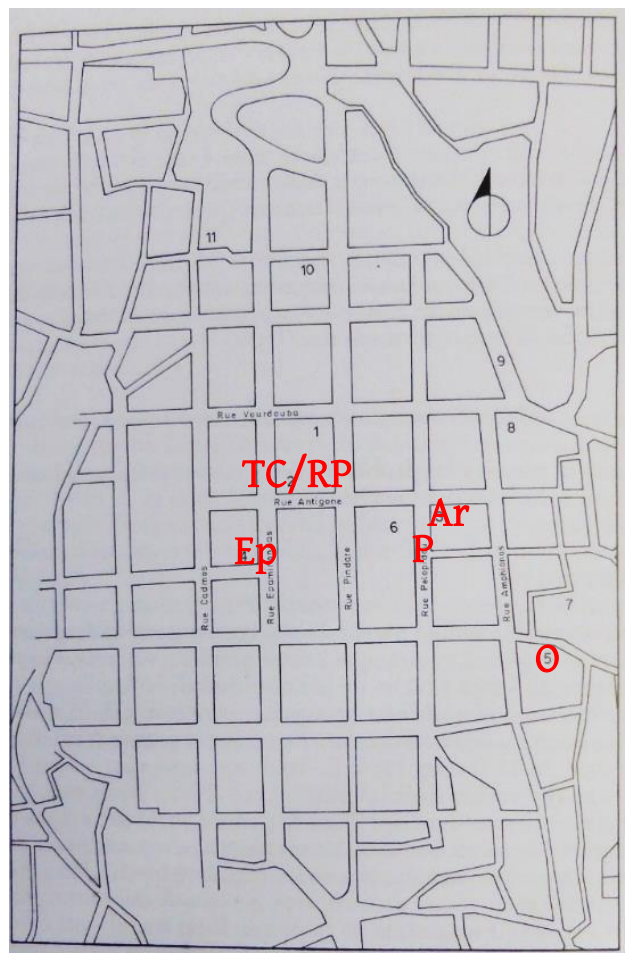

Of these deposits, the only ones with multiple examples of (an) undeciphered sign(s) are the Odos Pelopidou (P), a storeroom for agricultural products, ${ }^{654}$ and the Odos Oidipodos (O), a group of sealings, recording livestock and other associated products (Piteros et al. 1990). Other findspots include the Arsenal (Ar), so-called due to finds of weapons and pieces of harnesses (among other artefacts); ${ }^{655}$ the Odos Epameinondou (Ep), probably a 'clearinghouse' for the collection, storage, and disbursement of wool (Shelmerdine 1997:387-9; on the Of-series from this location, see also Del Freo-Rougemont 2012); and the adjoining Room of Pithoi (RP) and Treasure Chamber (TC), named for their finds of pits for storage vessels and precious objects (Dakouri-Hild 2001:83, 103-4).

\footnotetext{
${ }^{654} \mathrm{TFC}^{2}: 27-36,55-56$. A range of topics are represented in the $\mathrm{P}$ tablets, but chiefly distributions of grain and wine.

${ }^{655}$ On this location, and the Ug-series (which includes most of the tablets found here, and records an unknown commodity, 0), see most recently Del Freo 2014; other tablets from this findspot record textiles and agricultural produce.
} 


\section{Palaeography of the undeciphered signs at Thebes ${ }^{656}$}

$\underline{* 19}$ A $(\mathrm{p} .76)$

The single example of ${ }^{*} 19$ at Thebes (from TC; unattributed) is similar in its basic form to the Knossian examples.

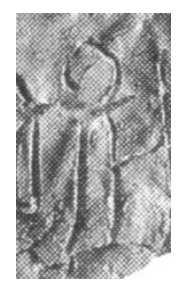

TH Wu $430 . \alpha^{657}$

\section{$\underline{22 / \mathrm{CAP}^{\wedge}}{ }^{\prime}(\mathrm{pp} .85,87)$}

This is the most widely-attested undeciphered sign at Thebes; it varies only in the form of the head:

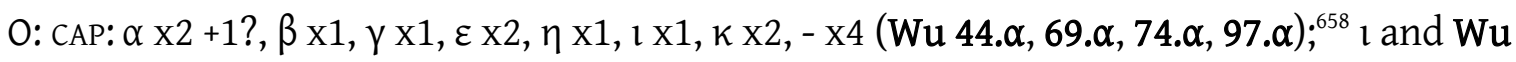
44. $\alpha$ have a vertical stroke, the others all have wavy heads P: *22: H305 x4+2; CAP: X1 (Uo 121.b, -); all with a vertical stroke (except H305's one unique 'hooked' example on Fq 214.12); note the differences in the shape of the head $\mathrm{RP}:{ }^{*} 22: \mathrm{x}$ ( (Uq 343.10, -)

TC: CAP X1̣ (Wu 431. $\alpha,-)$

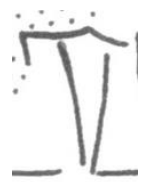

Fq 276.7 (H305)

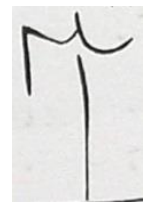

Uo $121(-) \quad$ Wu 54. $\alpha(\varepsilon)^{659}$

\footnotetext{
${ }^{656}$ The ISJs are excluded since they do not originate from Thebes (TSJ:92-4).

${ }^{657}$ Photo: TFC $^{3}$.

${ }^{658}$ It is not possible to securely identify scribal hands amongst the $O$ sealings due to the short length of their texts, but those with similar sign-forms have been placed into ten 'palaeographic groupings' of 2-5 sealings each, indicated by $\alpha-\kappa$ (Piteros et al. 1990:146; TFC ${ }^{3}$.

${ }^{659}$ Drawings: TFC $^{3}$.
} 
Both the RP and TC examples are excluded from the discussion below as their readings and forms are both uncertain or problematic (p.87, n.236).

*34

As at Pylos, the orientation of this sign is the only form of variation found here: H303? in

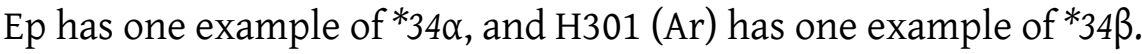

*63 F-1 (pp.123-6)

This sign is found only in P, where it is used by 3 identified scribes (H305, H306, H308). of these, H305 and H306 use Type 1 forms, and H308 uses Type 2 (as the unidentified author of Gf 163.3 probably also does).

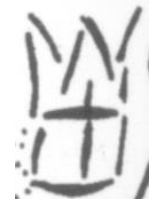

Fq $281.2(\mathrm{H} 305)$

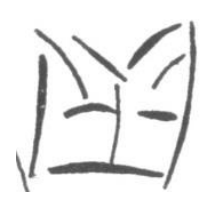

Gp $127.2(\mathrm{H} 306)$

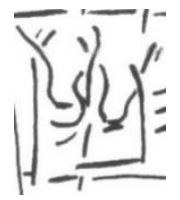

Gp $119.2(\mathrm{H} 308)^{660}$

\section{*65/FAR 休(p.137-8)}

This sign is found only in P, where it is used by 2-3 identified scribes (H305, H306;

perhaps H304). All of these examples are of Type 2, and although there is considerable variation in their exact forms (e.g. the relative length of strokes; the presence or absence of a lower horizontal), this occurs as much within individual hands as between different ones. This sign is therefore excluded from this study.

${ }^{660}$ Drawings: $T F C^{3}$. 


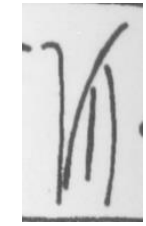

Fq 258.5 (H305)

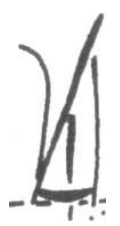

Fq 342.2 (H305)

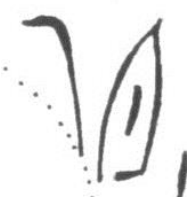

Gp $110.2(\mathrm{H} 306)$

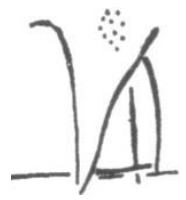

Gp $124.1(\mathrm{H} 306)^{661}$

\section{$\underline{\text { Discussion }}$}

Of the three signs which can be analysed for the purposes of this study, only *63 shows a distribution which stands a reasonable chance of being significant: both forms of this sign are found not only within a single deposit $(\mathrm{P})$, but also in hands who have written very similar tablets (H306 and H308, both responsible for Gp-series tablets recording wine distributions), whereas the two $\mathrm{P}$ hands who share the same form have no certain examples of tablets in the same series. ${ }^{662}$ Since only two examples of $*^{34}$ are attested, the fact that different forms are found in Ar and Ep may or may not be significant (and although the probability that this sign appears in the same MN, referring to the same individual, in both cases makes these deposits likely to be chronologically linked, it does not necessarily mean that they had a direct administrative connection) ${ }^{663}$ Likewise, the range of examples of *22/CAP in P (almost all in H305, apart from one unattributed example on Uo 121.b) is not great enough to say whether this sign's lack of variation in this deposit is significant (and note that, although all of these examples have a vertical stroke, the actual shape of the head is quite different in H305 and on Uo 121.b). The sealings from $\mathrm{O}$, which show a contrasting variation in the form of CAP, were presumably created and written in various different locations before being sent to the palatial centre

\footnotetext{
${ }^{661}$ Drawings: TFC $^{3}$.

${ }^{662} \mathrm{H} 305$ only certainly has Fq-series tablets; H306 only certainly has Gp-series tablets (see p.114, n.335).

${ }^{663}$ As seen on p.92, n.252, the MNs o-*34-ta(-o) and di-u-ja-wo/di-wi-ja-wo each occur as head of a textile workshop in $\mathrm{E}$ and in identical (if obscure) roles in transactions involving the unknown commodity $\mathrm{O}$ in $\mathrm{A}$, providing a strong contextual association between the two deposits. On the chronological implications of this, see pp.242-3.
} 
alongside the animals they recorded. ${ }^{664}$ Despite these sealings' apparent coherence in terms of their final administrative context and purpose (Piteros et al. 1990:172-84), this deposit cannot be treated as an administrative unit in the same way as the others.

\section{Conclusions}

Although little can be said about Theban administration within the context of this casestudy, there is no strong evidence against - and in the case of ${ }^{*} 63$, at least some positive evidence to support - the picture being essentially the same as in both Knossos and Pylos, that is, with little or no correlation between scribes' administrative relationships and their forms of the undeciphered signs.

\subsubsection{Mycenae}

Like Thebes, Mycenae has produced multiple tablet deposits in a wide range of locations, mostly in structures which are not physically part of the 'palace'; 665 unlike Thebes, however, the chronology of these deposits is relatively well-established (see further p.242) ${ }^{666}$ The only major deposits in which examples of the undeciphered signs are found are those from the 'Ivory Houses' - a group of four houses (the West House [WH], the House of the Oil Merchant [HOM], the House of the Sphinx [HS], and the House of the Shields) below the citadel ${ }^{667}$ whose destruction is dated to LH IIIB1; ${ }^{668}$ to these is added

\footnotetext{
${ }^{664}$ Animals are recorded as coming from at least two locations outside Thebes ( $a$-ma-ru-to and ka-ru-to: Piteros et al. 1990:153-5) and may of course have come from others which are not specified.

${ }^{665}$ However, at least some of these locations seem to have been connected to/controlled to some extent by the central palatial authority - in particular, the Ivory Houses are now generally regarded as part of the palatial administrative sphere (Tournavitou 1995:285-96; Shelmerdine 1997:390-4).

${ }^{666}$ Driessen 2008:74.

${ }^{667}$ See Tournavitou 1995.

${ }^{668}$ Driessen 2008:74.
} 


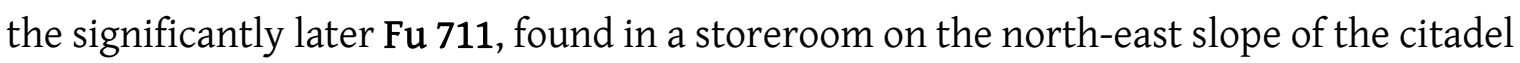
(NES; destruction dated to the end of LH IIIB) ${ }^{669}$

Palaeography of the undeciphered signs at Mycenae

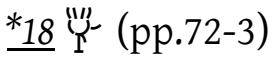

One possible example (H59; HS 6), which would be of Type 2, but cannot be certainly identified.

*47 笑

One very probable example (H57; HS 6) of the simpler type (with 3 small curves), with end-strokes.

\author{
Imageremoved \\ for copyright \\ reasons
}

\title{
Go $610.1^{670}$
}

*65/FAR ऋ

One example of *65 (H62; WH 1), of 'intermediate' type; two of FAR (Fu 711.7.8, -; NES), of the simpler Type 2 .

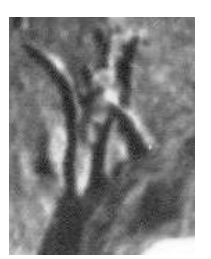

$\mathrm{Au} 653.6$

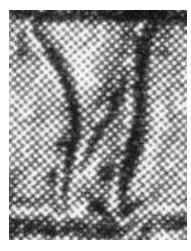

Fu 711.7.8 $(-)^{671}$

\footnotetext{
${ }^{669}$ Mylonas 1970.

${ }^{670}$ Photo: author (NAM).

${ }^{671}$ Photos: MER; SCM.
} 
*79

One example (H55; HOM 2) of the simpler type (a roughly oval shape).

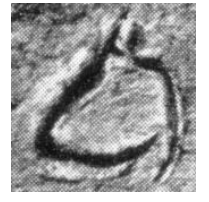

Oe $123^{672}$

$\underline{* 2}\}_{2}^{2}(\mathrm{p} .162)$

One certain example (H53; HOM 1) and one very probable (H61; WH 5). H53 has a Type 1 form (with a '3'-shaped central part), with a single curve to the right at top and bottom, but no elements to the left; what remains of H61's probable example suggests a similar form.

\section{Image removedfor copyright reasons}

Fo $101.2(\mathrm{H} 53)$

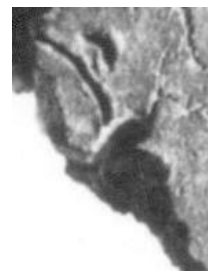

V $659.11(\mathrm{H} 61)^{673}$

The low number of examples of these signs makes it difficult to investigate any possible correlation between palaeography and administrative relationships. It is noticeable that in the case of a sign shared by two scribes from two of the Ivory Houses the scribes use the same form, ${ }^{674}$ while in the case of a sign shared between the Ivory Houses and the North-East Slope deposit there is at least a slight difference. Whether this is a significant

\footnotetext{
${ }^{672}$ Photo: SCM.

${ }^{673}$ Photos: author (NAM); MER.

${ }^{674}$ In fact, these probably appear in the same name, $a-n a^{-*} 82$, as several PNs are shared between Fo 101 (HOM) and V 659 (WH). For other examples of PNs recurring between these houses, see Tournavitou 1995:273-4.
} 
reflection of the close administrative relationship between the various Ivory Houses ${ }^{675}$ compared to their evident lack of connection with the later Fu 711 is, however, not possible to say given the lack of sufficient data.

\subsubsection{Conclusions}

This section has shown that, at least in the case of the undeciphered signs, there is no overall correlation between scribes' palaeographic similarities or differences and their administrative relationships. This is most clearly seen at Knossos and Pylos, which offer the best evidence for administrative reconstructions of this type, but the evidence from Thebes also appears to be compatible with this conclusion (what evidence there is from Mycenae may point in the opposite direction, but is too limited to be certainly significant). It is striking that despite the frequently-observed differences between the administrative systems and palaeography of Knossos and Pylos in particular, the picture which has emerged from this study is essentially similar; it is equally striking that this picture is a far more complex one than that suggested by any of the usual models of scribal work and training. The lack of correlation between palaeography and administrative relationships argues against scribes learning to write as 'apprentices' to scribes working in similar administrative areas; the wide range of variation displayed by some signs and similar lack of correlation between this and other forms of palaeographic classification (the Pylos classes; the Knossos ' $\mathrm{ni} / \mathrm{sa}$ ' division) does not seem to be particularly indicative of the existence of either 'scribal schools' or firmly-defined scribal traditions created by individuals responsible for scribes' training.

Of course, it should be stressed once again that these results are preliminary ones: a full palaeographic study of LB will be required in order to determine how representative this

\footnotetext{
${ }^{675}$ See Varias García 1993:67-8, 274-5, 332; Tournavitou 1995:263-5, 273-4, 286-7; Shelmerdine 1997:390-4.
} 
pattern, observed across a small sample of signs, really is. However, it is worth briefly exploring some of the wide range of potential implications which a similar result to these could have on our reconstructions of the scribes' work and training. A similar palaeographic situation could, for example, be produced by scribes tending either to be trained within larger and less specialised administrative areas - an environment in which relatively large numbers of scribes might at some point be involved in an individual's training - or to move between administrative areas and/or subjects in the course of their training (almost, to use a term that is just as anachronistic as 'school' or 'apprenticeship', like a system of 'internships'). The implication of such mobility or flexibility in the course of scribal training might then pose the question of how far this could also apply to the work of fully-fledged scribes: to what extent might the limited nature of our data (since each deposit of tablets probably represents at most only a few months' worth of documents immediately predating the destruction which preserved them) have obscured more complex scribal relationships and perhaps even exaggerated the scribes' apparent specialisations? Finally, what impact might this have on our view of the status of the scribes themselves - for instance, would a more mobile training and/or administrative system suggest that they administered a wider range of subjects, or subjects that were connected to each other in ways we cannot currently reconstruct? Or, alternatively, might it imply a status closer to that normally evoked by the term 'scribe' - that of a 'professional' writer and record-keeper, working for bureaucrats in different areas of the administration as necessary $?^{676}$

As yet, none of these suggestions can be more than speculative. However, this section has demonstrated that palaeographic analysis of individual scribes' forms of signs, when

\footnotetext{
${ }^{676}$ For 'scribes' as administrators, see, e.g., Bennet 2001:29-30; for 'scribes' as writers, see Palaima 2011:1223. For the suggestion that some scribes might also be craftspeople, see Kyriakidis 1996-1997:219-20.
} 
combined with archaeological data and administrative reconstructions, has the potential to make a vital new contribution to these unresolved questions concerning the Mycenaean scribes' work within the palatial administrative systems, their training, and perhaps even their status.

\subsection{Script development and chronology}

Palaeographic analysis has frequently been employed to trace the chronological development of LB, which is of interest not only for the history of the script itself, ${ }^{677}$ but more importantly as a potential method of establishing the relative chronology of different groups of tablets, whether between sites or within an individual site. ${ }^{678}$ The wider implications of this are clearly highly significant: in principle, this could provide a dating criterion either to supplement more standard archaeological means of dating finds (generally, in the case of LB tablets, through association with ceramics which can be typologically dated) or even to replace it when such archaeological information is not available or not clearly interpretable. The latter applies particularly to the site of Knossos, where - because much of the archaeological evidence relating to the dating of the site's LBA destruction(s) is highly controversial - arguments concerning the relationship between the various tablet deposits are often central to the chronological debate.

\footnotetext{
${ }^{677}$ E.g. Palaima 1988a uses a palaeographic comparison between LB and LA in an attempt to determine the date of LB's creation.

${ }^{678}$ This section is concerned only with relative chronology, expressed in terms of ceramic periods (e.g. LM/LH IIIA = Late Minoan/Late Helladic IIIA; during LM/LH III, the LM and LH periods are approximately equivalent, though the LH IIIA periods start slightly earlier than their LM equivalents: Shelmerdine 2008:4). For summaries of the ongoing debate over LBA Aegean absolute dating, see, e.g., Shelmerdine 2001:331-3 and 2008:3-7; Mountjoy 1999:16-18.
} 
At Knossos, proponents of the so-called 'unity of the archives' (i.e. the view that the majority of the tablets are contemporaneous $)^{679}$ rely on establishing links between deposits via features such as the presence of the same scribal hand or tablet-maker, or other apparent administrative connections, ${ }^{680}$ while the argument for the division of the Knossos tablets into several different time-periods depends on identifying different stages of LB palaeographic development in different tablet deposits. ${ }^{681}$ Palaeography has even affected the related debate over the date of the palace's 'final destruction', with comparisons to the LM IIIB1 Khania tablets being an important source of evidence for those arguing for a similar destruction date for Knossos, ${ }^{682}$ as opposed to the earlier possible date of LM IIIA1/2. ${ }^{683}$ The acceptance or dismissal of palaeographic analysis as a chronological tool would therefore have a significant impact on our understanding of the history not just of Knossos and its administration(s), but also of its relations with the

\footnotetext{
${ }^{679}$ It is important to note that 'archaeologically contemporary' deposits may or may not be 'historically contemporary': two deposits dated to the same ceramic period could in fact be separated by several decades. In comparing different sites, it is therefore possible only to make relatively broad-brush statements about their relative chronology. Within a single site, it may be possible to make more precise assignments of relative chronology between deposits: tablets from the same destruction level are in general likely to date from the same year (it is always possible that a few tablets left over from a preceding year could have been fired at the same time, but in general it appears unlikely that tablets were deliberately stored for much longer than a year: Bennet 2001:29-30). Tablets linked by features such as scribal hand are strictly speaking contemporary only to within that individual's working lifetime, but in practice the likelihood is again that they originate from the same year unless there is evidence for multiple destructions within a relatively short period.

${ }^{680}$ E.g. L.R. Palmer 1963a; Olivier 1967; Firth 1996-1997 and 2000-2001b; Greco 2012.

${ }^{681}$ Driessen 1997:130-2 and 2000:150-7; Skelton 2008 and 2011.

${ }^{682}$ Driessen 2000:152; on the comparison of the Khania tablets to Knossos H115, see also Olivier 1993; Palaima 1992-1993; Olivier 1996. For other arguments in favour of an LM IIIB1 date, see, e.g., L.R. Palmer 1963a; Hood 1965; E. Hallager 1977; Niemeier 1982; B.P. Hallager-Hallager 2015.

${ }^{683}$ See, e.g., Boardman 1963; Popham 1988 and 1997; Raison 1988 and 1993; Warren 1989. Since a full review of the highly controversial archaeological evidence is far beyond the scope of this thesis, I shall not take a position on this issue.
} 
rest of central and western Crete during the LBA, in addition to its potential implications for the prospect of dating further finds of tablets in unclear archaeological contexts. ${ }^{64}$ Despite the crucial importance of these questions for many aspects of Mycenaean studies, however, the fundamental assumptions underlying this chronological use of palaeography have never been explicitly investigated to any significant extent. The aim of this section is therefore to use the undeciphered signs as a test-case to investigate these assumptions. These signs' palaeography at Knossos will first of all be analysed relative to the possible chronological relationships between variant forms, with reference to links between different tablet deposits as well as Driessen's and Skelton's chronological classifications (p.232, n.681); this analysis will then be broadened to include the other sites already discussed in 3.1 in order to establish (as far as possible from the available evidence) not only how each individual sign developed over time, but also whether any overall patterns of development can be observed in this group of signs.

\subsubsection{Knossian internal chronology}

It is now widely (though not universally) (655 $^{6}$ accepted (following Driessen 1988, 1990, and 2000) that the RCT is likely to be significantly earlier in date than the rest of the Knossos tablets, a hypothesis which I follow here ${ }^{686}$ the main chronological debate at Knossos currently concerns the relationship between the major WW, EW, and NEP administrative areas. Based on reconstructions of administrative links (via scribal hands, palmprints, etc.), Firth 2000-2001b:268ff regards the tablets from these three areas as probably

\footnotetext{
${ }^{684} \mathrm{Cf}$. Shelmerdine 2012:76's comparison of the fairly broad date range suggested for the Iklaina tablet by the pottery associated with it in a refuse pit (LH IIB-IIIA2) with a palaeographic analysis suggesting similarities to the possibly LH IIIA Pylos H91 (p.241).

${ }^{685}$ See, e.g., Popham 1993.

${ }^{686}$ Driessen suggests a date of LM IIIA1 early (Driessen 2000:10, 220), revised from an earlier suggestion of LM II late (Driessen 1990:114).
} 
contemporary, ${ }^{687}$ while Greco 2012 argues for a chronological separation of just a few years between J1 and the NEP, based on textual analysis of the sheep records in each location. However, Driessen regards the NEP as significantly earlier than the main WW and EW areas (suggesting a tentative date of LM IIIA2 early, based on the assumption of a late date for the 'final destruction'). ${ }^{688}$ In my own opinion Firth's reconstruction appears the most plausible given the available evidence, since the reasonably high number of certain or probable links between the NEP and other deposits are hard to explain away; ${ }^{689}$ however, since the purpose of this section is to test the chronological potential of palaeography, I will generally treat the NEP as being of undetermined date relative to the WW and EW. As discussed above (pp.195-6), I also regard the E1 deposit as probably contemporary with the other WW tablets; however, the palaeographic implications of this potentially dating to a period between that of the RCT and the 'final destruction' (as argued by Driessen 1990:69-71 and 1997:120) will also be explored.

\footnotetext{
${ }^{687}$ The main exception is the small collection of D-series tablets from the WW, which Firth 1996-1997:33 argues may represent a previous year's records.

${ }^{688}$ Driessen 2008:76.

${ }^{689} \mathrm{H} 106, \mathrm{H} 120, \mathrm{H} 207$, and H217 were all found in the WW and NEP. H136, whose tablets have certainly been found in the WW and J1, probably provides a link between both of these locations and the NEP: a tablet attributed to H136? was found in the latter location, and one attributed to H136 was found in the adjacent I1, which is linked to the NEP via H125's V(5) 756 (this hand's V(5) set were mostly found in I3; V(5) 756 is recorded as found in I1. It is possible that this is a misrecording [Firth 2000-2001b:97]; however, this tablet shares the R Tau palmprint with other tablets from I1 [Sjöquist-Åström 1991: Table II], so would still provide a link between the two findspots). For other possible links via palmprints and prosopography, see Sjöquist-Åström 1991; Firth 1996-1997:68-9; Greco 2012. Driessen 2000:10-11 appears to suggest that there could be two chronological layers within the WW itself, one contemporary with the NEP and the other (including H103 and H115) with the EW (as well as Khania), but no detailed evidence is offered to differentiate these WW layers. Greco's explanation, while ingenious, relies heavily on the assumption that certain types of tablets were not preserved in each case to explain the difference between the sheep records of the NEP (mainly young animals and wool) and J1 (mainly adult animals) - a situation which seems easier to explain as the product of a division of labour within a contemporary administrative system.
} 
Two different palaeographic analyses have been carried out with a view to chronological separation of different Knossian tablet deposits, by Driessen 1997:130 and 2000:151-3 and Skelton 2008 and 2011, respectively. ${ }^{690}$ Although Skelton's analysis employs phylogenetic systematics (a method of reconstructing evolutionary relationships by analysing variation, taken from evolutionary biology), while Driessen's is a more 'traditional' palaeographic analysis, their conclusions are fairly similar: both divide the Knossos scribes into three groups, which are assigned a chronological as well as palaeographic significance. These groups are presented below in chronological order (hands in bold are those with examples of undeciphered signs, excluding *56; [?] indicates that an undeciphered sign is only tentatively attributed to that hand):

Driessen's groups: $:^{691}$

Conservative: 124 (RCT); 141, 222 (E1); ${ }^{692}$ 104, 106, 107, 109, 120, 123, 127, 137, 203 (NEP)

Centrist: 102, 110, 111, 112, 114, 118, 122, 125, 134, 201, 204 (NEP); 117, 119 (J1); 126 (J3); 136 (WW, NEP, J1); 202 (Arsenal)

Progressive: 101 (J2/3; B2); 105 (J2/3); 103, 108, 113, 115, 116[?], 121, 135, 140 (WW); 138, 139 (A); 128, 129, 130, 131, 132, 133[?] (Arsenal) ${ }^{693}$

Note that some secondary hands with examples of undeciphered signs are not classified under this analysis: 223 (WW), 224 (I1), 225 (NEP), and 217[?] (WW).

\footnotetext{
${ }^{690}$ The latter also includes other sites, on which see 3.2.2 (p.241ff).

${ }^{691}$ Driessen 2000:151-3.

${ }^{692} \mathrm{H} 141$ and $\mathrm{H} 222$ are regarded as a sub-category falling between the RCT and the rest of the 'conservative' group.

${ }^{693}$ In Driessen 2000:151, H113 and H139 are in fact listed as 'centrists', but are shown on the map on p.153 (and in Driessen 1997: fig.9) as 'progressive'; given his statements that the most progressive scribes are found in areas such as the WW and A, I assume that the latter is the intended classification.
} 
Skelton's groups (based on a sample of Knossian scribes): ${ }^{694}$

1) $\mathrm{RCT}^{695}$

2) $102 \mathrm{a},{ }^{696} 104,111,118$ (NEP); 141 (E1)

3) 101 (J2/3; B1); 103, 115 (WW); 117 (J1) (also KN V 52 $2^{697}$ and the Khania tablets)

Both groupings are thus in broad agreement, although Skelton's group 2 corresponds to a mixture of Driessen's conservative/centrist groups, and group 3 to the centrist/progressive groups: E1 and the NEP are thus classed as contemporary to each other and intermediate between the RCT and the main WW/EW areas.

Although the details of both Driessen's and Skelton's studies may be open to criticism, ${ }^{698}$ I am more concerned here with their methodologies. The application of phylogenetic systematics to a writing system, in particular, raises methodological questions as to its suitability for this purpose; ${ }^{699}$ but this is fundamentally based on similar underlying assumptions to Driessen's method, namely that palaeographic variation does have a chronological significance, and that this can be identified in a relatively straightforward way across the whole LB script. Admittedly, Driessen himself expresses the need for caution in assuming palaeographic evidence to have a chronological significance, with

\footnotetext{
${ }^{694}$ Skelton 2008:169-70, 172-3 and 2011:76-7.

${ }^{695} \mathrm{H} 124-\mathrm{B}, \mathrm{H} 124-\mathrm{R}$, and H124-S are included in this study.

${ }^{696}$ The classification of H102a (a sub-set of the hand classed by Olivier 1967 as H102) is attributed to R. Firth (Skelton 2008:162) but the reasoning behind this is not explained.

${ }^{697}$ Found in the RCT, but not belonging to H124 and of a different clay type from the other records: not regarded by Driessen 2000:20, n.8 as part of the RCT deposit proper.

${ }^{698}$ E.g. Driessen's study was carried out before the full publication of the Knossos tablets, and was based only on the sign-tables in Olivier 1967; Skelton's is based on only a small sample of scribes and tablets.

${ }^{699}$ This use of a form of analysis developed for biological evolutionary taxonomy naturally assumes that writing systems evolve in a similar way; but more complicated forms of development are clearly possible. Skelton 2008:160, for instance, points out the possibility of lateral transfer between contemporary script users; although such lateral transfer is not unknown in biology, this is not the kind of development which phylogenetic systematics is intended to analyse.
} 
the reminder that 'styles once introduced tend to persist side by side with both earlier and later fashions', ${ }^{700}$ and distinguishes in principle between 'palaeographic chronology' (i.e. the use of sign-forms with earlier or later origins in the history of the script) and archaeological chronology (e.g. Driessen 2000:152); nevertheless, his various publications do strongly imply that his palaeographic divisions are likely to have a real chronological significance, which is certainly how both his and Skelton's analysis are generally interpreted. ${ }^{701}$ Neither Driessen nor Skelton offers any detailed explanation of their methodology for selecting palaeographic features which are, or are not, thought to be chronologically significant, ${ }^{702}$ nor of which features are diagnostic of their different groupings, although Driessen 2000:151 states that his analysis is based on the principles of 'progressive change towards simplification and the shift of relative frequencies [presumably of different variants]'. The former assumption, in particular - that signforms will always tend to develop from a more elaborate form (in LA/early LB) to a simplified version - is one that is frequently made, ${ }^{703}$ but which has never been systematically investigated. It is therefore necessary to go back to the basic data and examine signs' variant forms while making as few chronological assumptions as possible, in order to test how far this assumption is justified. The questions to be addressed are twofold: 1) how far either of these classifications is an accurate reflection of

\footnotetext{
${ }^{700}$ Woodhead 1981:63, quoted in Driessen 2000:150.

${ }^{701}$ See, e.g., discussion in Killen 2015, or Shelmerdine 2012:76 on Iklaina (p.233, n.684).

${ }^{702}$ Skelton 2006 provides a list of the variant forms ('character states') used in her analysis, but without explanation as to the principle behind their selection. In some cases, types of variation which are diagnostic between scribes are omitted (e.g. for $* 47$, the presence or absence of the end-strokes are the only variation included), while in others, variation which is not diagnostic is included (e.g. for *65, the character states are the presence or absence of the bottom stroke, middle horizontal, and top horizontal: this obscures the similarities between forms with the same basic structure, while including features which may vary within a single scribal hand).

${ }^{703}$ See, e.g., Palaima 1988a:320: 'one observes a tendency of simplification of elaborate shapes when moving from the Knossos tablets...to the mainland tablets. I have always suspected these differences to have resulted from simplification through time, though this is beyond proof'.
} 
palaeographic groupings of scribal hands, and therefore useful in discussing processes of script development and palaeographic relationships between scribes; and 2) how far these classifications are compatible with the attested archaeological and epigraphic data and therefore likely to represent a real chronological division, insofar as the latter can be addressed within the scope of this chapter.

The distribution of variants of *49 and *79 fit exactly into Driessen's groupings: the 'centrist' H117 and H119 both have the form of *49 with extra strokes on the ends, while the 'progressive' H103 has the simpler form without them; likewise, H117 has a more elaborate form of *79 (which is also closer to AB79 and CH 005) than H103's simplified form. Similarly, the use of the extra stroke under the head of *22 (which is regular in LA) is found in some 'conservative' hands (H107 and some RCT hands) and sporadically in the 'centrist' H125 (fitting in with the general pattern of the gradual loss of this feature: p.89), while the end-strokes and more complex form of * 47 are found in all the 'conservative' hands who use this sign (H141, H222, and RCT hands) but in neither of the 'progressive' ones (H115 and H138) - although these features appear to have been LB developments rather than inherited (pp.102-3). *83 with central legs is found only in the 'conservative' group (RCT, H141, H222; although H141 may also have an example with right-hand legs), while the S-shaped form of the 'head' is found only in the 'progressive' H103 and H115.

However, the single- and double-stalk forms of ${ }^{*} 22$ are both found in all three groups, ${ }^{704}$ and the 1- and 2-stroke body forms of *83 both appear in the 'conservative' (RCT; H141,

\footnotetext{
${ }^{704}$ Scribes using *22: 'conservative': H120 (single), H124 (single and double); 'centrist': H117 (single and double), H122 (double), H125 (double); 'progressive': H101 (double), H103 (single), H105 (single), H108 (double), H116? (double), H121 (single), H132 (single), H133? (single).
} 
H222) and 'progressive' groups (H115; H103). ${ }^{705}$ Examples of 'centrist' hands having different forms (for instance, H114 and H117 differ in their forms of *34) may be less significant, since the 'centrist' class is by definition a hybrid one; the same may apply to examples where 'conservative' or 'progressive' hands share a form with 'centrist' ones, e.g. *18 (Type 1b is seen in 'centrist' H117 and H118 and 'progressive' H103 and H132). Other signs, however, appear to show exactly the opposite pattern to that assumed by Driessen: *65's forms remain similar in all three groupings with the exception of the single simplified form seen in a 'conservative' RCT hand, while the 'progressive' example of *82 (H103) has a more elaborate form (and one closer to AB82) than seen in the ‘centrist' H117's more simplified form; similarly, H117's form of *86 is arguably closer to CH 040 than the 'conservative' RCT form.

The chief points in which Skelton's groupings differ from Driessen's is in the positioning of H141 with H118 (the two being separated by Driessen as 'conservative' and 'centrist') and in the classification of H117 ('centrist') in Group 3 with other hands classed by Driessen as 'progressive'. However, although details of attributions vary, analysing these signs according to this grouping produces a picture which is essentially similar overall to that described above, or if anything displays even more overlap between groups and variation within groups. For instance, H103 and H117's different forms of *49, *79, and *82 would all belong in Group 3; the central-leg form of * 83 would be found in Groups 1 and 2 (RCT; H141), the latter of which would also contain examples with right-hand legs (H118), while Group 3 would include both the '3'- and S-shaped forms of the head (H117; H103, H115); both single- and double-stalk forms of ${ }^{*} 22$ would still appear in Group 1

\footnotetext{
${ }^{705}$ The appearance of multiple forms of the 'tail' within a group is less significant since this varies within single hands.
} 
(RCT: both) and Group 3 (H101: double; H103: single; H117: both; no Group 2 examples of this sign).

Overall, the distribution of the undeciphered signs' variant forms does not conform well to either of these palaeographic classifications. This is, of course, not necessarily significant, since it is possible that other signs might be more diagnostic in this regard; more problematic is the lack of any clear pattern of palaeographic development shown between the supposedly 'conservative'/earlier and 'progressive'/later forms. This can be compared with the evidence for probable links between different areas of the palace and the results of Section 1: the fact that in many cases multiple forms of individual signs were shown to be used within single deposits or closely-related administrative areas indicates that these were also in use during (approximately) the same time-period, and this is more broadly reflected in the fact that single administrative areas may contain scribes belonging to more than one of Driessen's groups. ${ }^{706}$ The NEP, for instance, contains both 'conservative' and 'centrist' hands; the WW is largely 'progressive', but contains one 'centrist' hand (H136) and probably also one or two 'conservative' hands (H141, H222), given that E1 was shown above (pp.195-6) to be likely to be related to the WW aromatics department (by the same argument, the WW would contain both Group 2 and 3 hands under Skelton's analysis). It is also worth reconsidering the evidence for links between these various different administrative areas: relatively strong links appear to exist between the WW and J1, particularly through the presence of H136 and perhaps H119; ${ }^{707}$ cf. p.234, n.689 on the similar links between the NEP and these two areas. If these

\footnotetext{
${ }^{706}$ Cf. E. Hallager 2015.

${ }^{707}$ Other possible links include finds of identical sealings (one set in G1 and J1; another in G1, G2, and J2, as well as B2/3 and K) and shared PNs, though these are less secure than scribal hands (the same PN does not necessarily represent the same individual; sealings may have remained in use for long periods of time: see Driessen 1997:125-7). However, considering the overall evidence, the division between sheep tablets in J1
} 
are accepted, all three of Driessen's groups, and Skelton's Groups 2 and 3, would be attested during the same chronological period. Even without accepting the connections between these areas, however, there is sufficient evidence to question the attribution of a real chronological significance to these palaeographic groupings.

\subsubsection{Inter-site relative chronology and script development}

Following on from this analysis of Knossos, this study will now be broadened to compare the chronological attestations of the undeciphered signs across different sites, whose chronology will first of all be briefly summarised..$^{708}$

Pylos: the vast majority of the tablets were either stratified within the palace's final destruction layer, which is now generally dated to the LH IIIB2/C transition, ${ }^{709}$ or (in the case of tablets displaced post-destruction) can be securely identified with it via, e.g., scribal hand attribution (Palaima 1988b:171). The only possible exception to this is the very small group of 'H91' and 'Class iv' tablets, found in areas of disturbed stratigraphy and assigned by Palaima 1983 and 1988b:111-3 to an earlier date (LH IIIA?) primarily on the basis of their perceived palaeographic similarity to Cretan LB (although the join of one 'H91' tablet, Xn 1449, to Vn 1335 [NEB] and the attribution of this whole tablet to Ciii [Melena 1996-1997b:165-6; PoNIV] calls into question the validity of this chronological distinction). There are, however, no examples of undeciphered signs in this group of tablets.

\footnotetext{
and textile production/storage tablets in the WW appears more likely to result from a contemporaneous division of labour than a chronological distinction (Firth 1996-1997 and 2000-2001b; Greco 2012).

${ }^{708}$ For a general summary of LB chronology, see Driessen 2008. ISJs are excluded here, since their medium and context of production may play at least as important a part in determining their palaeography as chronology (see Judson 2013:71-83). Note that this picture may change with the publication of the Ayios Vasileios tablets (provisionally dated to the LH IIIA1/2 transition: Vasilogamvrou 2015).

${ }^{709}$ Shelmerdine 2001:373, 381, n.325; Driessen 2008:73.
} 
Mycenae: the LB tablets date from several chronological periods between LH IIIA2 and the IIIB/C transition (summary in Driessen 2008:73-4); apart from Fu 711, dated to the end of LH IIIB (Mylonas 1970), all of the examples of undeciphered signs come from the LH IIIB1 Ivory Houses. ${ }^{710}$

Thebes: the majority of the tablets, including the Ep and P deposits, are dated to late LH IIIB2 or perhaps the IIIB2/C transition (TT II:53-5; $\left.T F C^{2}: 27-56\right)$. Other deposits were originally assigned earlier dates (Ar: LH IIIA2 $;^{711}$ O: LH IIIB1; ${ }^{712}$ RP/TC: LH IIIB1) ${ }^{713}$ but have more recently been argued to be also of LH IIIB2/C date (see especially $T F C^{2}: 57,237-44$ ). In the case of Ar, this redating seems plausible (though still unproven) due to the existence of a textual link to Ep (p.225, n.663) as well as probable associations with ; $^{714}$

\footnotetext{
${ }^{710}$ Iakovidis-French 2003:53-4.

${ }^{711}$ Platon-Stassinopoulou-Touloupa 1964a:896; TT I:116.

${ }^{712}$ Piteros et al. 1990:105.

${ }^{713}$ The original excavators of these two adjacent rooms variously identified the date of their destruction as either LH IIIB in general (Touloupa 1964:195; Touloupa-Symeonoglou 1965a:230) or LH IIIB1 (PlatonStassinopoulou-Touloupa 1964b:859); Aravantinos 2001:97 states that the more recent excavations show that the LB tablets belong to a slightly later phase, perhaps LH IIIB2. Subsequent publications refer to an LH IIIB2 date as certain for both rooms (e.g. TFC $2: 242,244$ ), but this seems to be based primarily on the Near Eastern cylinder seals found in the earlier excavations of the TC, which have been claimed to demonstrate a date of c.1225-1207 (e.g. $T F C^{1}$ :16) but in fact provide only a terminus post quem of c.1300 (i.e. near the beginning of LH IIIB: Dakouri-Hild 2001:103-4). For the moment I therefore prefer to class these deposits only as LH IIIB.

${ }^{714} \mathrm{TFC}^{2}: 241-3$ cite several pieces of evidence for this: 1) the buildings in Ar and P are similarly orientated, implying they may have formed part of the same complex; 2) fragments of LB tablets, including some joining to previously-discovered Ug-series tablets from Ar, were recently found alongside sherds identified as LH IIIB2 in drawers containing finds from the original excavation; 3) the 1994 excavations in Ar produced two LB tablets (Lf 139, Ft 140) from the same stratigraphic context as the P tablets, and two PNs from $\mathrm{Ft} 140$ are also found in P (o-ke-u-ri-jo, Av 104.3; e-re-o-ni, X 155); 4) the close similarity in form of some Ar and P tablets. None of these are unproblematic: 1) does not prove the contemporaneity of their tablet deposits; 2) the pottery remains unpublished and the possibility of post-excavation contamination of finds from different layers remains to be disproven; 3 ) is contradicted by other reports which refer to these tablets being found in a disturbed context (e.g. Aravantinos 2005:28) and contextual evidence to prove identity of these PNs is lacking; 4) is hardly conclusive. However, taking all this together, along with the fact that some reports from the original excavation also imply that the tablets were found alongside
} 
however, no such links exist for the other deposits, ${ }^{715}$ and their chronology cannot be properly evaluated pending full publication of the relevant excavations.

The table below gives an approximate comparison of these sites' chronology with that of Knossos. When a deposit's chronology is debated, both possibilities are given; the possible chronological range of deposits assigned to a fairly broad period is indicated by dashes. Note also that, as said above (p.231, n.678), LH IIIA1 and A2 may in fact start slightly earlier than their LM counterparts.

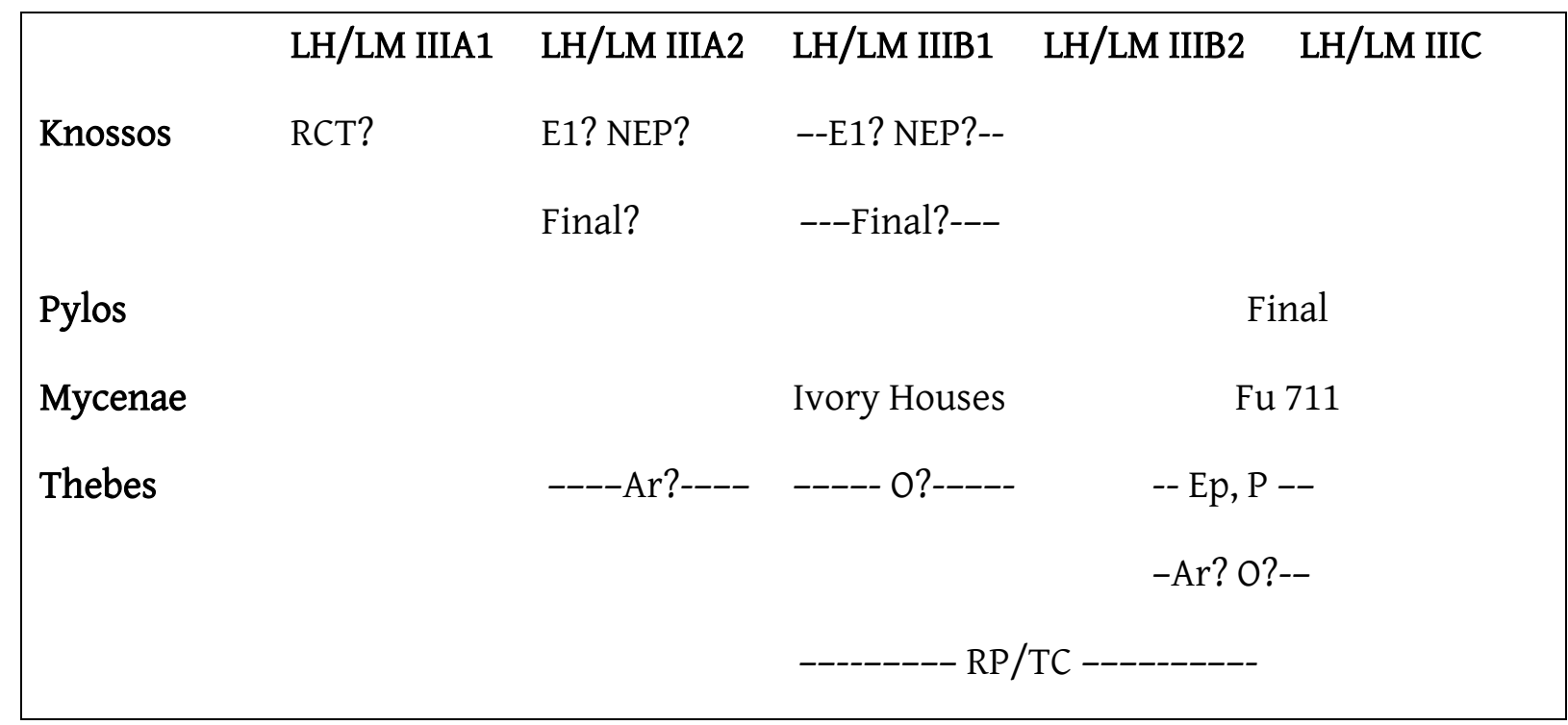

LH IIIB, rather than LH IIIA, pottery (Touloupa-Symeonoglou 1965b:233; Ioannidou 1971:209), there seems to be at least a reasonable probability that the Ar tablets should, like P, be dated to LH IIIB2 late or the IIIB2/C transition.

${ }^{715} T^{2} C^{2}: 239-42$ suggest that $a$-e-ri-qo on Uq 434 (RP) and Wu 70 and 76 (0) refers to a single person (see also Aravantinos et al. 2008:28-30). However, the fact that in both cases $a-e-r i-q o$ is making a contribution in fulfilment of an o-pa obligation (of a hide on Uq 434 and a cow on Wu 76; on Wu 70 a-e-ri-qo contributes a sheep with no qualification) does not seem a strong enough piece of contextual evidence to make this identification; an o-pa is not an uncommon type of obligation (in the 560 sealings, at least 5 individuals are recorded as contributing under an o-pa), and there are probably two contemporary individuals with this name recorded at Pylos (Nakassis 2013:192). 
The most important point to note is that, however the Knossian 'final destruction' is dated, it is still significantly earlier than both the Pylos tablets and the Thebes tablets (at least those from Ep and P, which may be contemporary with, or slightly earlier than, Pylos). This, along with the probability that the RCT is significantly earlier than the rest of the Knossian tablets, provides the best prospects of chronological comparison, although other deposits whose relative chronology is less certain will also be included in the discussion.

This kind of chronological comparison between sites raises methodological problems of its own, since it is entirely possible that different sites could have undergone different processes of script development; were an LM IIIA1 deposit to exist at Pylos, for instance, we have no way of knowing how far it would resemble the RCT palaeographically. ${ }^{716}$ In the current state of the evidence, however, it is impossible to attempt any chronological analysis of LB without such inter-site comparisons. Likewise, the comparison of LB forms with their LA and $\mathrm{CH}$ correspondences raises even greater methodological difficulties, since this involves comparing forms not only from different sites, but also across different media and types of inscriptions; both LA and $\mathrm{CH}$ are themselves attested across a wide chronological range ${ }^{717}$ not to mention the problem of the relationship between these two scripts and the possible palaeographic effects of the process of LB's creation. ${ }^{718}$ Nonetheless, in the absence of attestations of LB over a longer time-period, comparison with LA or CH is the only way to provide information about possible very early forms; this will therefore be included subject to certain restrictions (e.g. LA and $\mathrm{CH}$ forms on

\footnotetext{
${ }^{716} \mathrm{Cf}$. the frequent statement of the differences between mainland and Knossian/Cretan LB: e.g. Bennett 1966; Duhoux 1987.

${ }^{717}$ LA texts are attested from MM II-LM IIIA, although the majority fall within the period MM III-LM IB (for a recent summary, see Del Freo-Zurbach 2011:75, 84-5). CH texts are attested from MM I-LM IB, with the majority from MM II-MM III (CHIC:27-32; Tsipopoulou-Hallager 1996:7 and 2010:26).

${ }^{718}$ See in general Palaima 1988a.
} 
clay documents are more likely to be comparable to $\mathrm{LB})^{719}$ and to the caveat that LA and CH forms cannot be straightforwardly identified with the 'original' LB forms.

As stated above (p.237), it is often assumed that the general tendency is for LB signs to develop from a more elaborate form (in LA and/or early LB) to a more simplified or stylised form. ${ }^{720}$ All of the undeciphered signs show a contrast between elaborate forms and simpler ones which could in principle have developed from the simplification of the former:

*18 18 : Type 1 vs. Type 2, which requires fewer strokes; Type 1 also includes the more elaborate $1 \mathrm{a}$ and simpler $1 \mathrm{~b}$ *19 A: forms with/without a central vertical *22/CAP $\uparrow \mid$ : forms with/without the small stroke under the head; forms with a vertical stroke at the end of the head vs. a wavy head

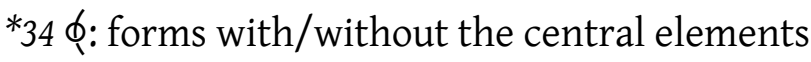
${ }^{*} 47$ N: double/single central element; with/without end-strokes

*49 $\mathbb{A}$ : with/without the small cross-strokes

*56||: forms with four/three/two horizontals

*63 FI: elaborate curving top vs. triangular top

*64 [ख्र: with/without strokes on the ends of the vertical

*65/FAR K: elaborate Type 1 vs. simpler Type 2

*79,

*82 $\int_{2}^{2}:$ ' 3 '-shaped vs. simple curve; more/fewer strokes to left or curves to right

\footnotetext{
${ }^{719}$ In the discussion below, standardised forms of signs are given for illustrative purposes, but those based on examples from clay documents rather than other media have been selected (especially in the case of $\mathrm{CH}$, in which forms on seals may differ significantly from those on clay). For further images, see the relevant sections of Chapter 2.

${ }^{720}$ E.g. Palaima 1988a:320; Driessen 2000:151.
} 
*83 梁: forms with central/symmetrical legs vs. only right-hand legs; form with one righthand leg instead of two; '3'- or ' $\mathrm{S}$ '-shaped head vs. small curves or straight strokes; more or fewer strokes forming tail or body *86 Ł : with/without extra strokes at the top of the vertical or the right-hand end; with/without the curve from the top to the right-hand end

Examining the chronology of these forms' attestations, however, shows that the majority are not straightforward examples of simplification over time.

Although the Knossian Type 2 examples of 18 紧- are of unknown findspot, they are very unlikely to be later than the Type 1 examples from the (probably contemporary) WW and J1, which include both Types $1 \mathrm{a}$ and $1 \mathrm{~b}$ (even found within a single hand, H117); the NEP example of Type $1 b$ is either earlier than or contemporary to the J1 Type 1a.

The examples of *19 \& with a central vertical are found only at Pylos; the simpler Theban example (TC) cannot be later than these (and is probably at least slightly earlier), and the simpler Knossian examples (including one from the RCT) are certainly earlier.

The use of the small stroke under the head of ${ }^{*} 22 / \mathrm{CAP}^{\wedge} \mid$ ' does seem to fit the expected pattern, since this is present in the majority of LA forms ( $\uparrow)$, found at Knossos only in the early RCT and the possibly intermediate NEP, and absent from the later mainland deposits; but in all cases (even in LA) the form without this stroke is also attested, so that the most that can be said is that a deposit with a greater proportion of scribes using this stroke may have a greater chance of being relatively early in date. ${ }^{721}$ Conversely,

\footnotetext{
${ }^{721}$ Note, however, that this feature is also found on Cretan ISJs, all probably dating to LM IIIB; it is hard to say whether this should therefore be considered a Cretan/mainland distinction rather than a
} 
however, the form with a vertical stroke at the end of the head - arguably a more complex one, requiring an extra stroke to write compared to the wavy-head form - is sporadically present in LA and likewise at Knossos (where it is not diagnostic), but is much more frequent in the later mainland deposits ( $O$ and $\mathrm{P}$ at Thebes; Pylos, where it is clearly diagnostic). Again, this is generally attested alongside the wavy-head form (the exception, P at Thebes, has no examples attributed to hands other than H305). Although the single-stalk forms of ${ }^{*} 22$ are simpler than the double-stalk forms on the same principle (requiring one fewer stroke), they are unlikely to have originated as such (since these correspond to different forms of CAP); although H117's use of the double-stalk form in headings and the single-stalk elsewhere demonstrates that the latter could be regarded as a simpler equivalent to the former, there is no overall trend to be seen in the use of either form across time (the RCT, NEP, J1, and WW all have both; Thebes only certainly has the double-stalk, but all certain examples of *22 are in a single hand).

*34 $\phi$ appears to show increasing elaboration over time: only the simplest forms are attested in LA $(<\in$ ) and the RCT, and only the more elaborate forms in later deposits (Knossos: NEP, J1; Pylos; Thebes: Ar, Ep), although the lack of early elaborate forms could be due to chances of attestation given the low number of examples of AB34 and of *34 from the RCT. The sign's orientation has no chronological significance: ${ }^{*} 34 \alpha$ and ${ }^{*} 34 \beta$ are contemporaneous at Pylos and perhaps at Knossos (NEP, J1) and Thebes (Ar, Ep); the absence of $* 34 \beta$ from the RCT (and from LA) is probably due to chance given the low number of attestations in each and the fact that $*_{34 \alpha}$ is the more common orientation.

chronological one, or whether the ISJ medium has encouraged the retention of a more archaic/elaborate feature. 
*47 has a complex chronological distribution. Its form with an elaborate centre appears in the RCT and E1, which is either roughly contemporary with the NEP or, more probably, with the WW; either way, however, this is attested at the same period as the simpler centre, which is found in I1 (adjacent to the NEP, and probably linked to it) ${ }^{722}$ and the WW. The very probable example at Mycenae (either contemporary with or later than the WW examples) has the simpler centre but with end-strokes, which are also found on all other examples except those from the WW. Moreover, both these end-strokes and the more elaborate centre may be LB developments since they are unparalleled in AB47 $\not \varnothing$.

Although the form of ${ }^{49} \mathbb{\mathbb { A }}$ without the extra strokes near the ends of the verticals may be a simplification in LB, since these are present on all of the (few) examples of AB49 $\overparen{F}$, this is probably attested at the same time as the form with these strokes, since J1 and the WW are probably contemporary (pp.233-4).

The simplified two-horizontal form of ${ }^{*} 56$ 非 is a rare, idiosyncratic form at Knossos (H117: J1) and Thebes (H309: P), not even diagnostic of scribal hand; the elaborated fourhorizontal form is equally idiosyncratic (H111: NEP). No particular chronological significance can therefore be attached to this.

The elaborate (Type 1) and intermediate forms of *63 FF are found contemporaneously at Pylos, and the Type 1 and simplified Type 2 forms are likewise found at Thebes (in P).

Knossos has only the more elaborate form of ${ }^{6} 64$ [ (including one RCT example), but both this and the simpler form are attested simultaneously at Pylos. While the lack of the

\footnotetext{
${ }^{722}$ See p.234, n.689.
} 
simpler forms at Knossos may therefore be chronologically significant, it may equally be due to chance since there are only two examples at this site; the presence of the more elaborate forms is certainly not chronologically diagnostic.

The simpler Type 2 form of*65/FAR W is the closest one to AB65 怆, appearing at Knossos only in the RCT, but is used by almost as many scribes at Pylos as the more elaborate Type 1; Thebes (P) has only this form, while Mycenae has an earlier intermediate example (also paralleled at Pylos) and a later Type 2 example (approximately contemporary with Pylos). Whether or not the absence of Type 1 examples from these last two sites is significant, here we seem to have an example of an originally simpler LA form becoming more elaborate in LB, after which the simplified form apparently dropped out of use at Knossos but persisted on the mainland.

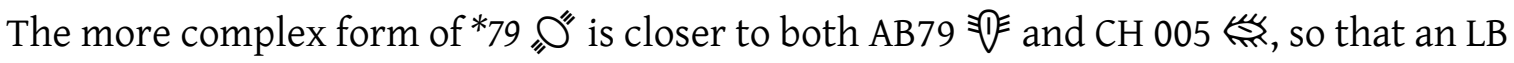
simplification seems likely; however, it is probable that both Knossos and Pylos have both forms of *79 attested contemporaneously - Knossos in J1 and the WW; at Pylos, the example of the simpler form is uncertain but probable (Mycenae has only the simpler form, from the Ivory Houses, but with only one example this is not necessarily significant).

The more elaborate Type 1 form of * 82$\}_{2}^{2}$ is similarly closer to AB82 繁, but Knossos has both Type 1 and an intermediate form probably attested contemporaneously (WW and J1), while Pylos has both Type 1 and Type 2 (the former being the only one found in Mycenae's Ivory Houses). As with *64, the lack of the simpler forms at Knossos may be chronologically significant (but may equally be due to chance), but the presence of the 
more elaborate forms is certainly not chronologically diagnostic. ${ }^{723}$ In addition, comparison with $k u$ suggests that the variation between a 3-shape and a curve may well originate from LA, with the lack of LA examples of the latter being due to chance. There is also no chronological pattern to be found in the elements to either side of ${ }^{*} 82$, which seem to be quite particular to individual scribes.

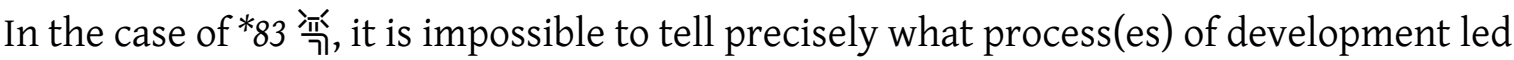
to the various different forms attested - whether the Knossian forms with 'legs' only on the right-hand side are simplifications of a form similar to the Knossian central-leg form ${ }^{724}$ and/or the Pylian symmetrical one, or the latter are elaborations of the former. The only relatively clear case of simplification is seen within Pylos itself, where the forms with only one, shorter, right-hand 'leg' appear to be simplified versions of the symmetrical form, and both of these are of course contemporary. The more elaborate and simpler forms of the 'head' may both appear even within individual hands; no clear chronological pattern emerges regarding these or the other forms of variation displayed by this sign.

A fairly drastic simplification process appears to have taken place to produce ${ }^{*} 86$

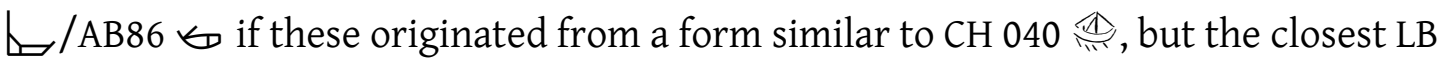
parallel to AB86 is the simpler examples from Pylos, while of the two Knossian examples, the later H117 (J1) form $₫$ is more similar to $\mathrm{CH}$ than the RCT example.

\footnotetext{
${ }^{723}$ Note that the Type 2 example on the Cretan ISJ TH Z 853 shows that this cannot simply be a mainland development.

${ }^{724}$ Found only in the RCT and E1, but as already argued the latter is probably contemporary with examples of the right-hand leg form from the WW.
} 


\subsubsection{Conclusions}

Although there are a reasonable number of cases amongst the undeciphered signs where a relatively straightforward process of palaeographic development (from LA to LB and/or within LB) can be plausibly reconstructed, these include processes of elaboration as well as simplification over time; and in other cases, signs vary in ways which cannot easily be fitted into this kind of progressive developmental framework. Moreover, there are very few instances where the actual chronology of forms' attestations fits in with these reconstructed palaeographic processes; it is much more common for forms which may be palaeographically 'earlier' or 'later' to be attested contemporaneously in at least one site or deposit, and in several cases LB seems to be continuing or modifying patterns of variation originating (at least) in LA.

Of course, any overall palaeographic and/or chronological classification must be founded on the basis of general tendencies amongst scribal hands, not purely on the basis of individual forms; it is entirely possible that a full palaeographic study of LB would reveal processes of development with a much clearer chronological significance. Nonetheless, in demonstrating that the assumption that LB signs will generally simplify over time is unsound on several counts, these results suggest that the usual model of script development is over-simplistic and in need of re-examination; until such a reexamination has taken place, the use of palaeographic analysis to assign a 'real' relative chronology to groups of tablets is therefore also highly questionable.

\subsection{Case-study conclusions}

Both parts of this case-study have produced results which have called into question widespread assumptions about the LB script and its use. This investigation of the palaeography of the undeciphered signs has shown that even within this small group of 
signs, the palaeographic situation is much more complex than expected, whether examining variation within a single site or across different sites; this palaeographic complexity has implications reaching far beyond the study of the LB script itself.

The demonstration that some of the fundamental assumptions underlying the chronological use of palaeography are unfounded does not show that palaeography cannot be used in this way; it does, however, show that our current understanding of the processes of palaeographic change which took place in LB and their possible chronological significance is inadequate for this purpose, and chronological arguments based wholly or partly on such evidence should be regarded with considerable scepticism. This applies to the use of inter-site palaeographic comparisons - in particular the comparison of KN H115 to the Khania tablets and the consequent dating of the 'final destruction' of Knossos to LM IIIB1 - as much as, or even more than, to the identification of different chronological layers within a single site (e.g. the various possible layers at Knossos, or Class iv/H91 at Pylos). Given that multiple styles of writing have been shown to co-exist at individual sites and even within single deposits, the same is surely possible across different sites, particularly ones whose relationship is as unclear as that of LM IIIB1 Knossos and Khania. ${ }^{725}$ While certainly not demonstrating that the 'final destruction' of Knossos took place earlier than LM IIIB1, this study does show the need to revisit the arguments, both palaeographic and archaeological, for this late dating, whose

\footnotetext{
${ }^{725}$ For the view that LM IIIB1 Khania was an independent kingdom, see (e.g.) Haskell 1983 and 1986; Palaima 1984; Godart-Tzedakis 1989:407 and 1992:330-5. For the view that Knossos was still in control of West Crete at this point, see (e.g.) L.R. Palmer 1971:85; Driessen 1996:1015-6 and 2000:9-11. E. HallagerHallager 2011:431 provides a summary of the evidence relating to Khania as an administrative centre; Driessen-Farnoux 1991:90-2 summarises the debate focusing on the ISJs. Note also that the Khaniote scribe concerned is currently attested on only two relatively fragmentary tablets in fairly poor condition (Ar 4 and Gq 5) with, respectively, only 18 and 6 different securely-identified signs; unless or until further tablets attributable to this hand are found in Khania, the evidence is very slight compared to the weight of arguments placed on it, even if this chronological use of palaeography were shown to be valid.
} 
acceptance or dismissal would have a profound impact on our understanding of the history of LBA Crete.

The comparison of the Mycenaean scribes' palaeographic similarities to their reconstructable administrative relationships, which showed no correlation between these two features, similarly challenges the frequent assumption that scribes were trained in 'apprenticeships' within the administrative areas they would go on to work in; the high level of variation in the forms of these signs and the lack of a close correspondence between this and wider palaeographic groupings is equally problematic for the alternative assumptions of 'scribal schools' or training by a limited number of identifiable individuals. In this case, the range of possible implications relating to the scribes' work, training, and status is clearly very wide, and a limited case-study such as this does not allow firm conclusions to be drawn on these issues. Like the chronology study, however, these results both point to the need for further investigation of LB palaeography and demonstrate its wide-ranging potential to address broader issues within the field of Mycenology. 


\section{$\underline{\text { Overall conclusions }}$}

With respect to the central question relating to the LB undeciphered signs - that of their sound-values - the conclusions of this thesis are, to some extent, fairly negative ones. As Chapter 2 demonstrated, not only is the state of the evidence currently insufficient to propose a decipherment for the majority of the undeciphered signs, but many proposed sound-values which have been relatively widely accepted are also at best unverifiable and at worse highly implausible. Such conclusions are, however, of critical importance not just in correcting misconceptions about these signs' status of decipherment, but also in enabling further research to take place on a more secure footing. Combining a detailed study of each individual sign's corpus of attestations with an analysis of the relative probability of different sound-values and their likely properties has enabled considerable progress to be made in establishing which types of sound-values are the most likely for each sign. Thus Chapter 2 has also made a much more positive contribution to this question in establishing the best possible basis, given the current state of the evidence, for any future attempts at decipherment, particularly if or when new examples of these signs come to light.

However, the results of this thesis also have a much wider significance beyond the question of the undeciphered signs' possible sound-values. For instance, our understanding of issues such as the history of the LB and LA scripts and the nature of the Minoan language has been hindered by widespread assumptions concerning the development of LB, in particular the presumed Minoan origins of the extra signs: decipherment proposals which rely on these assumptions have then been used in turn to further reinforce them in arguments relating to the whole LB script. As Chapter 1 demonstrated, cutting through this series of circular arguments by showing these original assumptions to be unfounded puts us in a better position both to identify 
probable values for the undeciphered signs and to understand the history of $\mathrm{LB}$, its relationship with LA, and the possibility of reconstructing Minoan linguistic features. Moreover, a detailed examination of individual signs' origins and usage has enabled an exploration of the processes of LB script development, in particular the motivations underlying the creation of new signs and the crucial impact a script's context of use - in the case of LB, for the specific purposes of the Mycenaean administrations - can have on the decisions made by its users in the course of its development.

Chapter 3 has taken this exploration of LB's context of use still further, addressing issues of even wider importance for the study of Mycenaean history and society as well as of the LB texts. Employing the undeciphered signs as a palaeographic case-study has enabled the development of a methodology for further investigation of the links between palaeography and administration and of the chronological significance of variation in sign-forms: this case-study therefore provides a basis for future work in these areas. Extending this methodology to the entire LB script will enable a full, systematic analysis of script development and the potential of palaeography to be used in determining tablets' relative chronology. Equally, when combined with similar investigations of other aspects of the Mycenaean scribes' writing practices such as orthography, this analysis will be able to more fully address the questions and possibilities raised by this case-study relating to the scribes' work, training, interrelationships, and status. This study of the LB undeciphered signs has therefore produced results whose implications go far beyond the question of these signs' possible sound-values, and which will eventually have a significant impact on the wider study of Mycenaean history and society as a whole. 
Bibliographic abbreviations

CDE Chantraine, P. (1968), Dictionnaire étymologique de la langue grecque: histoire des mots (Paris: Éditions Klincksieck)

CHIC Olivier, J.-P., \& Godart, L. (1995), Corpus Hieroglyphicarum Inscriptionum Cretae, École française d'Athènes: Études crétoises 31 (Paris: de Boccard)

CIV Sacconi, A. (1974a), Corpus delle iscrizioni vascolari in lineare B, Istituto per gli studi micenei ed egeo-anatolici: Incunabula Graeca LVII (Roma: Edizioni dell'Ateneo)

COMIK Chadwick, J., Godart, L., Killen, J.T., Olivier, J.-P. et al. (1986-1998), Corpus of Mycenaean Inscriptions from Knossos vol. I-IV, Istituto per gli studi micenei ed egeoanatolici: Incunabula Graeca LXXXVIII (Cambridge/Roma: Cambridge University Press/Edizioni dell'Ateneo)

CTLA Raison, J., \& Pope, M. (1994), Corpus transnuméré du linéaire A, Bibliothèque des Cahiers de l'Institut de Linguistique de Louvain 74 (Louvain-la-Neuve: Peeters)

DĀMOS Aurora, F., Nesøen, A., Nedić, D., Løken, H. et al., (2013), 'DĀMOS - Database of Mycenaean at Oslo', https://www2.hf.uio.no/damos/. Last updated: 7.11.2015 University of Oslo

DMic Aura Jorro, F. (1985-1993), Diccionario micénico vol. I-II, Diccionario griego-español (Madrid: Instituto de Filología)

Docs ${ }^{1}$ Ventris, M.G.F., \& Chadwick, J. (1956), Documents in Mycenaean Greek, $1^{\text {st }}$ edn. (Cambridge: Cambridge University Press)

Docs $^{2}$--- (1973), Documents in Mycenaean Greek, $2^{\text {nd }}$ edn. (Cambridge: Cambridge University Press)

EDG Beekes, R.S.P. (2010), Etymological Dictionary of Greek, Leiden Indo-European Etymological Dictionaries 10.1-2 (Leiden/Boston: Brill)

GORILA Godart, L., \& Olivier, J.-P. (1976), Recueil des inscriptions en linéaire A, École française d'Athènes: Études crétoises 21 (Paris: P. Geuthner)

$K T^{4}$ Chadwick, J., Killen, J.T., \& Olivier, J.-P. (1971), The Knossos Tablets, $4^{\text {th }}$ edn. (Cambridge: Cambridge University Press) 
KT Killen, J.T., \& Olivier, J.-P. (1989), The Knossos Tablets, 5th edn., Suplementos a Minos 11 (Salamanca: Universidad de Salamanca)

LSJ Liddell, H.G., Scott, R., Jones, H.S., McKenzie, R. et al. (1996), A Greek-English Lexicon, 9th edn. (Oxford: Clarendon Press)

MT II Bennett, E.L., Wace, A.J.B., Wace, E.B., \& Chadwick, J. (1958), 'The Mycenae Tablets II', Transactions of the American Philosophical Society 48:1, 1-122

MT III Chadwick, J., Bennett, E.L., Jr., French, E.B., Verdelis, N.M. et al. (1962), ‘The Mycenae Tablets III', Transactions of the American Philosophical Society 52:7, 1-76

MT IV Olivier, J.-P. (1969b), The Mycenae Tablets IV: a revised transliteration (Leiden: Brill)

PoNIV Bennett, E.L., Melena, J.L., Olivier, J.-P., Palaima, T.G. et al. (2013 [unpublished]), The Palace of Nestor at Pylos in Western Messenia vol. IV: The Inscribed Documents (Texas University Press for the University of Cincinnati), www.academia.edu/5788888/

PT II Bennett, E.L. (1955), The Pylos Tablets: Texts of the Inscriptions Found 1939-1954 (Princeton: Princeton University Press for the University of Cincinnati)

PTT Bennett, E.L., \& Olivier, J.-P. (1973), The Pylos Tablets Transcribed, Istituto per gli studi micenei ed egeo-anatolici: Incunabula Graeca LI \& LIX (Roma: Edizioni dell'Ateneo)

SCM Sacconi, A. (1974b), Corpus delle iscrizioni in lineare B di Micene, Istituto per gli studi micenei ed egeo-anatolici: Incunabula Graeca LVIII (Roma: Edizioni dell'Ateneo)

TFC Aravantinos, V.L., Godart, L., Sacconi, A., Andrikou, E. et al. (2001-2005), Thèbes. Fouilles de la Cadmée vol. I-IV, Biblioteca di "Pasiphae" (Pisa/Roma: Istituti editoriali e poligrafici internazionali)

TFC ${ }^{1}$ Aravantinos, V.L., Godart, L., \& Sacconi, A. (2001), Thèbes. Fouilles de la Cadmée I. Les tablettes en linéaire B de la Odos Pelopidou. Édition et commentaire, Biblioteca di "Pasiphae" I (Pisa/Roma: Istituti editoriali e poligrafici internazionali)

TFC ${ }^{2}$ Andrikou, E., Aravantinos, V.L., Godart, L., Sacconi, A. et al. (2006), Thèbes. Fouilles de la Cadmée II.2. Les tablettes en linéaire B de la Odos Pelopidou: Le contexte archéologique. 
La céramique de la Odos Pelopidou et la chronologie du linéaire B, Biblioteca di "Pasiphae" II.2 (Pisa/Roma: Instituti editoriali e poligrafici internazionali)

TFC ${ }^{3}$ Aravantinos, V.L., Godart, L., \& Sacconi, A. (2002), Thèbes. Fouilles de la Cadmée III. Corpus des documents d'archives en linéaire B de Thèbes, Biblioteca di "Pasiphae" III (Pisa/Roma: Istituti editoriali e poligrafici internazionale)

TFC ${ }^{4}$ Aravantinos, V.L., Godart, L., Sacconi, A., \& Del Freo, M. (2005), Thèbes. Fouilles de la Cadmée IV. Les textes de Thèbes (1-433). Translitération et tableaux des scribes, Biblioteca di "Pasiphae" IV (Pisa/Roma: Istituti editoriali e poligrafici internazionali)

TITHEMY Melena, J.L., \& Olivier, J.-P. (1991), TITHEMY: the Tablets and Nodules in Linear B from Tiryns, Thebes and Mycenae: a Revised Transliteration, Suplementos a Minos 12 (Salamanca: Universidad de Salamanca)

TSJ Haskell, H.W., Jones, R.E., Day, P.M., \& Killen, J.T. (2011), Transport Stirrup Jars of the Bronze Age Aegean and East Mediterranean (Philadelphia: INSTAP Academic Press)

TT I Chadwick, J. (1970), 'Linear B tablets from Thebes', Minos 10:2, 115-37

TT II Spyropoulos, T.G., \& Chadwick, J. (1975), The Thebes Tablets II, Suplementos a Minos 4 (Salamanca: Universidad de Salamanca)

UPSID Maddieson, I., Precoda, K., \& Reetz, H., 'UCLA Phonological Segment Inventory Database (UPSID)', web.phonetik.uni-frankfurt.de/upsid.html, retrieved: October 2015 Institut für Phonetik, Universität Frankfurt

VIP Raison, J. (1968), Les vases à inscriptions peintes de l'âge mycénien et leur contexte archéologique, Istituto per gli studi micenei ed egeo-anatolici: Incunabula Graeca XIX (Roma: Edizioni dell'Ateneo)

Younger (CH) Younger, J.G., 'The Cretan Hieroglyphic texts: a web edition of the texts based on CHIC with commentary', www.people.ku.edu/ jyounger/Hiero/index.html, retrieved: 11/03/2016. Last updated: 23/09/2013

Younger (LA) ---, 'Linear A texts in phonetic transcription and commentary: introduction', www.people.ku.edu/ jyounger/LinearA/, retrieved: 11/03/2016. Last updated: 09/12/2015 
Younger (LA HT) ---, 'Linear A texts in phonetic transcription: Haghia Triada', www.people.ku.edu/ jyounger/LinearA/HTtexts.html, retrieved: 11/03/2016. Last updated: 09/12/2015

Younger (LA Lexicon) ---, 'Linear A lexicon', www.people.ku.edu/ jyounger/LinearA/lexicon.html, retrieved: 11/03/2016. Last updated: 09/12/2015

Younger (LA Other) ---, 'Linear A texts in phonetic transcription: other texts (not Haghia Triada)', www.people.ku.edu/ jyounger/LinearA/misctexts.html, retrieved: 11/03/2016. Last updated: 05/10/2015

Other bibliography

Åkerström, Å. (1978), 'Mycenaean problems: I. On the Mycenaean chariot', Opuscula Atheniensia 12:3, 19-37

Arapogianni, P., Rambach, J., \& Godart, L. (1999), 'L’inscription en linéaire B de Kafkania Olympie (OL Zh 1)', in Deger-Jalkotzy, S., Hiller, S. \& Panagl, O. (eds) Floreant Studia Mycenaea. Akten des X. internationalen mykenologischen Colloquiums in Salzburg vom 15 Mai 1995 vol. I (Wien: Verlag der Österreichischen Akademie der Wissenschaften), 39-43

Aravantinos, V.L. (2001), 'Contenu, contexte et fonction du "trésor" du palais mycénien de Thèbes (Béotie): une approche économique et administrative', in Carlier, $\mathrm{P}$. (ed.) Journées égéennes. Nanterres, 8-10 mars 1999, Ktèma: Civilisations de l'Orient, de la Grèce et de Rome antiques 26 (Strasbourg: Université Marc Bloch), 87-100

--- (2005), 'La tavolette frammentaria TH Ug 43. Un interessante caso di conflitto tra dati di scavo e dati epigrafici', in Adembri, B. (ed.) AEIMNHSTOE. Miscellanea di studi per Mauro Cristofani vol. 1, Prospettiva supplemento 2 (Firenze: Centro Di), 25-31

Aravantinos, V.L., Godart, L., \& Sacconi, A. (1995), 'Sui novi testi del palazzo di Cadmo a Tebe', Rendiconti dell'Accademia Nazionale dei Lincei. Classe di scienze morali, storiche e filologiche, serie IX VI:4, 809-45

--- (2004), 'En marge des nouvelles tablettes en linéaire B de Thèbes', Kadmos 42, 15-30

--- (2006), ‘Commentaires aux nouveaux textes insérés dans le corpus de Thèbes', in Deger-Jalkotzy, S. \& Panagl, O. (eds) Die neuen Linear B-Texte aus Theben. Ihr 
Aufschlusswert für die mykenische Sprache und Kultur. Akten des internationalen Forschungskolloquiums an der Österreichischen Akademie der Wissenschaften, 5.-6. Dezember 2002 (Wien: Verlag der Österreichischen Akademie der Wissenschaften), $1-9$

--- (2008), 'La tavoletta TH Uq 434', in Sacconi, A., Del Freo, M., Godart, L. \& Negri, M. (eds) Colloquium Romanum. Atti del XII colloquio internazionale di micenologia, Roma 2025 febbraio 2006 vol. I, Pasiphae I (Pisa/Roma: Fabrizio Serra), 23-33

Aravantinos, V.L., \& Vasilogamvrou, A. (2012), 'The first Linear B documents from Ayios Vasileios (Laconia)', in Carlier, P., de Lamberterie, C., Egetmeyer, M., Guilleux, N. et al. (eds) Études mycéniennes 2010. Actes du XIIIe colloque international sur les textes mycéniens et égéens. Paris 20-23 septembre 2010, Biblioteca di "Pasiphae" X (Pisa/Roma: Fabrizio Serra), 41-54

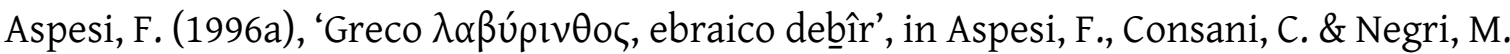

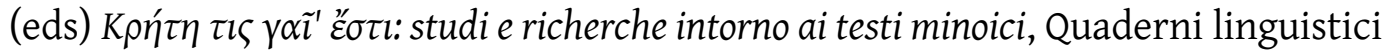
e filologici, Università di Macerata, Facoltà di lettere e filosofia, Istituto di glottologia e linguistica generale VII (Roma: Editrice 'il Calamo'), 147-81

--- (1996b), 'Lineare A (-)du-pu - -re: un'ipotesi', in Aspesi, F., Consani, C. \& Negri, M. (eds)

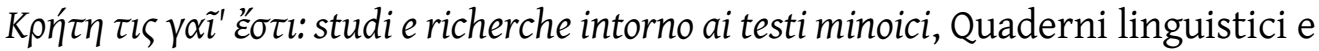
filologici, Università di Macerata, Facoltà di lettere e filosofia, Istituto di glottologia e linguistica generale VII (Roma: Editrice 'il Calamo'), 137-45 [II incontro internazionale di linguistica greca (Trento, 29-30 settembre 1995)]

Barber, E.J.W. (1991), Prehistoric Textiles: The Development of Cloth in the Neolithic and Bronze Ages with Special Reference to the Aegean (Princeton: Princeton University Press)

Bartoněk, A. (2003), Handbuch des mykenischen Griechisch, Indogermanische Bibliothek. Erste Reihe (Heidelberg: Winter)

Baumbach, L. (1972), 'Further thoughts on PY Vn 46', in Ruipérez, M.S. (ed.) Acta Mycenaea. Proceedings of the Fifth International Colloquium on Mycenaean Studies, held in Salamanca, 30 March - 3 April 1970 vol. II, Minos 12 (Salamanca: Universidad de Salamanca), 383-97

--- (1986), 'The personal names on the Knossos Ap tablets', in Etter, A. (ed.) o-o-pe-ro-si. Festschrift für Ernst Risch zum 75. Geburtstag (Berlin/New York: de Gruyter), 273-8 
--- (1987), 'Names of shepherds at Knossos', Acta Classica 30, 5-10

--- (1992), 'The people of Knossos: further thoughts on some of the personal names', in Olivier, J.-P. (ed.) Mykenaika: actes du IXe Colloque international sur les textes mycéniens et égéens organisé par le Centre de l'Antiquité Grecque et Romaine de la Fondation Hellénique des Recherches Scientifiques et l'École française d'Athènes (Athènes, 2-6 octobre 1990), Bulletin de correspondance hellénique. Supplément 25 (Athens: École française d'Athènes), 57-64

Beekes, R.S.P. (2007), 'Pre-Greek', Glotta 83, 13-17

--- (2014), Pre Greek: Phonology, Morphology, Lexicon (edited by S. Norbruis), Brill Introductions to Indo-European Languages 2 (Leiden/Boston: Brill)

Bendall, L.M. (2003), 'A reconsideration of the Northeastern Building at Pylos: evidence for a Mycenaean redistributive center', American Journal of Archaeology 107:2, 181231

--- (2014), 'Gifts to the goddesses: Pylian perfumed olive oil abroad?', in Nakassis, D., Gulizio, J. \& James, S.A. (eds) KE-RA-ME-JA. Studies Presented to Cynthia W. Shelmerdine, Prehistory Monographs 46 (Philadelphia: INSTAP Academic Press), $141-62$

Bennet, J. (1998), 'The Linear B archives and the kingdom of Nestor', in Davis, J.L. (ed.) Sandy Pylos. An Archaeological History from Nestor to Navarino (Austin: University of Texas Press), 111-33

--- (2001), 'Agency and bureaucracy: thoughts on the nature and extent of administration in Bronze Age Pylos', in Voutsaki, S. \& Killen, J.T. (eds) Economy and Politics in the Mycenaean Palace States: Proceedings of a Conference Held on 1-3 July 1999 in the Faculty of Classics, Cambridge, Cambridge Philological Society. Supplementary volume 27 (Cambridge: Cambridge Philological Society), 25-37

--- (2008), 'Palace ${ }^{\mathrm{TM}}$ : speculation on palatial production in Mycenaean Greece with (some) reference to glass', in Jackson, C.M. \& Wager, E.C. (eds) Vitreous Materials in the Late Bronze Age Aegean, Sheffield Studies in Aegean Archaeology 9 (Oxford: Oxbow), $151-72$

--- (2011), 'The geography of the Mycenaean kingdoms', in Duhoux, Y. \& Morpurgo Davies, A. (eds) A Companion to Linear B. Mycenaean Greek Texts and their World vol. 
II, Bibliothèque des Cahiers de l'Institut de Linguistique de Louvain 127 (Louvainla-Neuve: Peeters), 137-68

Bennett, E.L. (1966), 'Some local differences in the Linear B script', Hesperia: The Journal of the American School of Classical Studies at Athens 35:4, 295-309

--- (1972), 'Linear B sematographic signs’, in Ruipérez, M.S. (ed.) Acta Mycenaea. Proceedings of the Fifth International Colloquium on Mycenaean Studies, held in Salamanca, 30 March - 3 April 1970 vol. I, Minos 11 (Salamanca: Universidad de Salamanca), 55-72

--- (1992), 'A selection of Pylos tablet texts', in Olivier, J.-P. (ed.) Mykenaïka: actes du IXe Colloque international sur les textes mycéniens et égéens organisé par le Centre de l'Antiquité Grecque et Romaine de la Fondation Hellénique des Recherches Scientifiques et l'École française d'Athènes (Athènes, 2-6 octobre 1990), Bulletin de correspondance hellénique. Supplément 25 (Athens: École française d'Athènes), 103-27

Bernabé, A. (2008), 'Some thoughts on the Knossos Ra series', in Sacconi, A., Del Freo, M., Godart, L. \& Negri, M. (eds) Colloquium Romanum. Atti del XII colloquio internazionale di micenologia, Roma 20-25 febbraio 2006 vol. I, Pasiphae I (Pisa/Roma: Fabrizio Serra), 111-20

Bernabé, A., Alonso, J.L., Benito, L.M., Cantarero, R. et al. (1992-1993), 'Estudios sobre el vocabulario micénico II: términos referidos a los carros', Minos 27-28, 125-66

Bernabé, A., \& Luján, E.R. (2006), Introducción al griego micénico: gramática, selección de textos y glosario, Monografías de filología griega 18 (Zaragoza: Prensas Universitarias de Zaragoza)

Bibee, W.E., \& Wilson-Wright, A.M. (2015), 'A laryngeal in Linear B: evidence from Ugaritic and Egyptian' (Paper read at Aegean Scripts. 14th International Colloquium on Mycenaean Studies, held in Copenhagen, 2-5 September 2015)

Blegen, C.W., \& Lang, M. (1960), 'The Palace of Nestor excavations of 1959', American Journal of Archaeology 64:2, 153-64

Boardman, J. (1963), 'The date of the Knossos tablets', in Boardman, J. \& Palmer, L.R. (eds) On the Knossos Tablets (Oxford: Clarendon Press) 


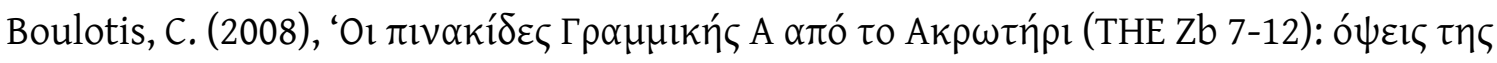

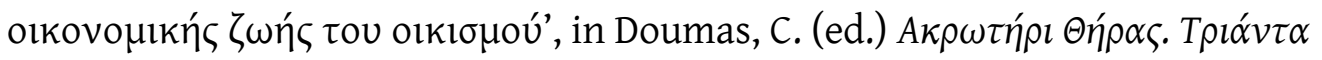

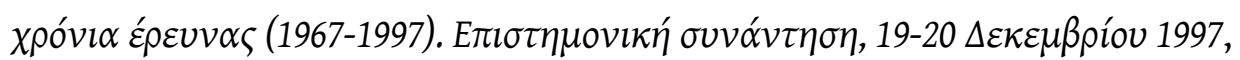

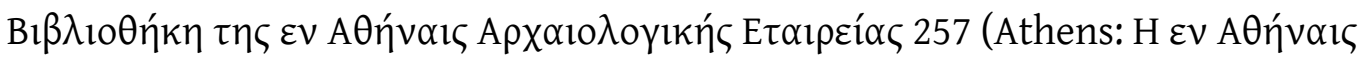

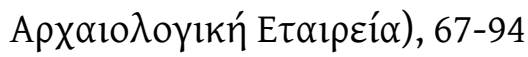

Buck, C.D. (1955), The Greek dialects: grammar, selected inscriptions, glossary (Chicago: University of Chicago Press)

Camera, C. (1968), 'Una presunta scuola degli scrivani a Cnosso', Studi micenei ed egeoanatolici 7, 116-28

Carlier, P. (1984), La royauté en Grèce avant Alexandre, Études et Travaux publiés par le Groupe de Recherche d'Histoire romaine de l'Université des Sciences Humaines de Strasbourg VI (Strasbourg: AECR)

Chadwick, J. (1956), 'Problèmes d'interprétation du linéaire B', in Lejeune, M. (ed.) Études mycéniennes. Actes du colloque international sur les textes mycéniens. Gif-sur-Yvette, 3-7 avril 1956 (Paris: Centre national de la recherche scientifique), 237-47

--- (1957 [unpublished]), 'Sign *64' (Paper read at the Minoan Linear B Seminar: Minutes of the Meeting of November 13th 1957, University of London, Institute of Classical Studies; Mycenaean Epigraphy Room, Faculty of Classics, Cambridge)

--- (1958), 'Error and abnormality in the Mycenaean noun-declension', Parola del passato LXII, 285-91

--- (1967), 'The archive of the Room of the Chariot Tablets at Knossos (proceedings of the Mycenaean Seminar)', Bulletin of the Institute of Classical Studies 14, 103-4

--- (1968a), ‘The group sw in Mycenaean', Minos 9, 62-5

--- (1968b), 'The organisation of the Mycenaean archives', in Bartonĕk, A. (ed.) Studia Mycenaea. Proceedings of the Mycenaean Symposium, Brno, April 1966 (Brno: Universita J.E. Purkyně), 11-21

--- (1983), 'Traditional spelling or two dialects', in Heubeck, A. \& Neumann, G. (eds) Res Mycenaeae. Akten des VII. internationalen mykenologischen Colloquiums in Nürnberg vom 6.-10. April 1981 (Göttingen: Vandenhoeck \& Ruprecht), 78-88 
--- (1988), 'The women of Pylos', in Olivier, J.-P. \& Palaima, T.G. (eds) Texts, Tablets and Scribes. Studies in Mycenaean Epigraphy and Economy offered to Emmett L. Bennett, Jr., Suplementos a Minos 10 (Salamanca: Universidad de Salamanca), 43-95

--- (1996-1997), ‘Three temporal clauses’, Minos 31-32, 293-301

Chadwick, J., \& Killen, J.T. (1965), 'On the reading of the Knossos tablets', Klio 46, 93-102

Chadwick, J., \& Ventris, M.G.F. (1953), 'Greek records in the Minoan script', Antiquity 27:108, 196-206

Chantraine, P. (1933), La formation des noms en grec ancien, Collection linguistique publiée par la société de linguistique de Paris XXXVIII (Paris: Librairie ancienne Honoré Champion)

--- (1947), Morphologie historique du grec, Nouvelle Collection à l'Usage des Classes XXXIV (Paris: Librairie C. Klinksieck)

Civitillo, M. (2008), 'Il sillabogramma *19: status quaestionis e proposte di lettura', in Sacconi, A., Del Freo, M., Godart, L. \& Negri, M. (eds) Colloquium Romanum. Atti del XII colloquio internazionale di micenologia, Roma 20-25 febbraio 2006 vol. I, Pasiphae I (Pisa/Roma: Fabrizio Serra), 131-50

Consani, C. (1981), 'Per la definizione del valore fonetico del sillabogramma *56 nel sillabario lineare B', Rendiconti dell'Accademia Nazionale dei Lincei. Classe di scienze morali, storiche e filologiche XXXVI:1-2, 9-18

--- (1996), 'AB 118/DWO tra minoico e miceneo', in Consani, C., Negri, M. \& Aspesi, F.

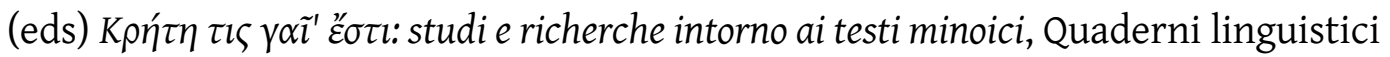
e filologici, Università di Macerata, Facoltà di lettere e filosofia, Istituto di glottologia e linguistica generale VIII (Roma: Editrice 'il Calamo'), 71-81

Cosmopoulos, M.B. (2006), 'The political landscape of Mycenaean states: a-pu $\mathrm{pu}_{2}$ and the Hither Province of Pylos', American Journal of Archaeology 110:2, 205-28

Crouwel, J.H. (1981), Chariots and Other Means of Land Transport in Bronze Age Greece, Allard Pierson Series Studies in Ancient Civilisation 3 (Amsterdam: Allard Pierson) 
Dakouri-Hild, A. (2001), 'The House of Kadmos in Mycenaean Thebes reconsidered: architecture, chronology, and context', The Annual of the British School at Athens 96, $81-122$

--- (2010), 'Thebes', in Cline, E.H. (ed.) The Oxford Handbook of the Bronze Age Aegean (ca. 3000-1000 BC) (Oxford: Oxford University Press), 690-711

Davis, B. (2014), Minoan Stone Vessels with Linear A Inscriptions, Aegaeum 36 (Leuven/Liège: Peeters)

de Venuto, D. (1968), 'Il segno 82 del sillabario micenico', in Atti e memorie del $1^{\circ}$ congresso internazionale di micenologia (Roma 27 settembre-3 ottobre 1967) vol. II, Incunabula Graeca XXV (Roma: Edizioni dell'Ateneo), 580-7

Del Freo, M. (2014), 'Observations on the Thebes Ug series', Kadmos 53, 45-70

Del Freo, M., \& Rougemont, F. (2012), 'Observations sur la série Of de Thèbes’, Studi micenei ed egeo-anatolici 54, 263-80

Del Freo, M., \& Zurbach, J. (2011), 'La préparation d'un supplément au Recueil des inscriptions en linéaire A. Obsérvations à partir d'un travail en cours', Bulletin de correspondance hellénique 135, 75-97

Demakopoulou, K., Divari-Valakou, N., Schallin, A.-L., Ekroth, G. et al. (2002), 'Excavations in Midea 2000 and 2001', Opuscula Atheniensia 27, 27-58

Dimopoulou, N., Olivier, J.-P., \& Réthémiotakis, G. (1993), 'Une statuette en argile MR IIIA de Poros/Irakliou avec inscription en linéaire A', Bulletin de correspondance hellénique 117:2, 501-21

Doria, M. (1958), 'I - À propos de quelques problèmes de phonétique du grec ancien à la lumière des données mycéniennes. II - Nouvelle interprétation du signe 82', in Atti del $2^{\circ}$ colloquio internazionale di studi minoico-micenei. Pavia, 1-5 IX 1958, Athenaeum. Studi periodici di letteratura e storia dell'antichità XLVI - Nuova Serie XXXVI - Fasc. IV (Pavia: Athenaeum), 95-104 [389-98]

--- (1959), 'Il valore del segno 66 del sillabario miceneo', Parola del passato LXIV, 5-25

--- (1962), 'Nuove riflessioni sul sistema ortografico miceneo', Atti dell'Istituto Veneto di scienze, lettere ed arti CXX, 643-75 
--- (1970), 'Review of J.T. Killen - J.P. Olivier, '155 raccords de fragments dans les tablettes de Cnossos', Bulletin de correspondance hellénique 92 (1968)', Studi micenei ed egeo-anatolici XI, 155-6

--- (1972), 'Sur la difficulté d'établir avec certitude la valeur de certains signes syllabiques "rares" du linéaire B', in Ruipérez, M.S. (ed.) Acta Mycenaea. Proceedings of the Fifth International Colloquium on Mycenaean Studies, held in Salamanca, 30 March - 3 April 1970 vol. II, Minos 12 (Salamanca: Universidad de Salamanca), 33-51

Doumet-Serhal, C., Karageorghis, V., Loffet, H., \& Coldstream, N. (2008), 'The kingdom of Sidon and its Mediterranean connections', in Doumet-Serhal, C., Rabate, A. \& Resek, A. (eds) Networking Patterns of the Bronze and Iron Age Levant: the Lebanon and its Mediterranean Connections. On the Occasion of the Symposium 'Interconnections in the Eastern Mediterranean: the Lebanon in the Bronze and Iron Ages', 4-9 November 2008, Beirut, Archaeology \& History in the Lebanon (London: Lebanese British Friends of the National Museum), 1-70

Driessen, J. (1988), 'The scribes of the "Room of the Chariot Tablets"', in Olivier, J.-P. \& Palaima, T.G. (eds) Texts, Tablets and Scribes. Studies in Mycenaean Epigraphy and Economy offered to Emmett L. Bennett, Jr., Suplementos a Minos 10 (Salamanca: Universidad de Salamanca), 123-65

--- (1990), An Early Destruction in the Mycenaean Palace at Knossos: a New Interpretation of the Excavation Field-Notes of the South-East Area of the West Wing, Acta archaeologica Lovaniensia. Monographiae 2 (Leuven: Katholieke Universiteit Leuven)

--- (1996), 'Architectural context, administration and political architecture in Mycenaean Greece', in de Miro, E., Godart, L. \& Sacconi, A. (eds) Atti e memorie del secondo congresso internazionale di micenologia, Roma-Napoli, 14-20 Ottobre 1991 vol. II: Storia (Rome: Gruppo editoriale internazionale), 1013-28

--- (1997), 'Le palais de Cnossos au MR II-III: combien de destructions?', in Driessen, J. \& Farnoux, A. (eds) La Crète mycénienne. Actes de la Table Ronde internationale organisée par l'École française d'Athènes, 26-28 mars 1991, Bulletin de correspondance hellénique. Supplément 30 (Athens: École française d'Athènes), 113-34

--- (1999), 'The Northern Entrance Passage at Knossos. Some preliminary observations on its potential role as 'central archives", in Deger-Jalkotzy, S., Hiller, S. \& Panagl, O. (eds) Floreant Studia Mycenaea. Akten des X. internationalen mykenologischen 
Colloquiums in Salzburg vom 1-5 Mai 1995 vol. I (Wien: Verlag der Österreichischen Akademie der Wissenschaften), 205-26

--- (2000), The Scribes of the Room of the Chariot Tablets, Suplementos a Minos 15 (Salamanca: Universidad de Salamanca)

--- (2008), 'Chronology of the Linear B texts', in Morpurgo-Davies, A. \& Duhoux, Y. (eds) A Companion to Linear B: Mycenaean Greek Texts and their World vol. I, Bibliothèque des Cahiers de l'Institut de Linguistique de Louvain 120 (Louvain-la-Neuve: Peeters), 69-79

Driessen, J., \& Farnoux, A. (1991), 'Painted Linear B inscriptions from Malia', Bulletin de correspondance hellénique 115:1, 71-93

Duhoux, Y. (1978), 'Une analyse linguistique du linéaire A', in Duhoux, Y. (ed.) Études minoennes I. Le linéaire A, Bibliothèque des Cahiers de l'Institut de Linguistique de Louvain 14 (Louvain-la-Neuve: Peeters), 65-129

--- (1983), 'Les syllabogrammes 34 et 35 du linéaire B', in Heubeck, A. \& Neumann, G. (eds) Res Mycenaeae. Akten des VII. internationalen mykenologischen Colloquiums in Nürnberg vom 6.-10. April 1981 (Göttingen: Vandenhoeck \& Ruprecht), 112-25

--- (1986), ‘The teaching of orthography in Mycenaean Pylos', Kadmos 25, 147-54

--- (1987), 'Linéaire B crétois et continental: éléments de comparaison', in Ilievski, P. \& Crepajcie, L. (eds) Tractata Mycenaea. Proceedings of the Eighth International Colloquium on Mycenaean Studies, held in Ohrid, 15-20 September 1985 (Skopje: Macedonian Academy of Sciences and Arts), 105-28

--- (1989), 'Le linéaire A: problèmes de déchiffrement', in Duhoux, Y., Palaima, T.G. \& Bennet, J. (eds) Problems in Decipherment, Bibliothèque des Cahiers de l'Institut de Linguistique de Louvain 49 (Louvain-la-Neuve: Peeters), 59-120

--- (1998), 'Pre-hellenic language(s) of Crete', The Journal of Indo-European Studies 26, 1-39

--- (1999), 'La séparation des mots en linéaire B', in Deger-Jalkotzy, S., Hiller, S. \& Panagl, O. (eds) Floreant Studia Mycenaea. Akten des X. internationalen mykenologischen Colloquiums in Salzburg vom 1-5 Mai 1995 vol. I (Wien: Verlag der Österreichischen Akademie der Wissenschaften), 227-36 
--- (2007), 'Le nom du 'fils' en linéaire B', in Lang, F., Reinholdt, C. \& Weilhartner, J. (eds)

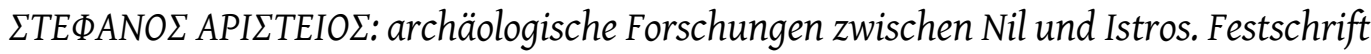
für Stefan Hiller zum 65. Geburtstag (Wien: Phoibos), 95-102

--- (2008), 'Mycenaean anthology', in Duhoux, Y. \& Morpurgo Davies, A. (eds) A Companion to Linear B: Mycenaean Greek Texts and their World vol. I, Bibliothèque des Cahiers de l'Institut de Linguistique de Louvain 120 (Louvain-la-Neuve: Peeters), 243-393

--- (2011), 'How were the Mycenaean scribes taught?', Pasiphae: Rivista di filologia e antichità egee V. Proceedings of the International Colloquium "The Inner Workings of Mycenaean Bureaucracy", University of Kent, Canterbury, 19-21 September 2008, 95-118

Faure, P. (1979), 'Review of Louis Godart et Jean-Pierre Olivier, Recueil des inscriptions en linéaire A (sigle GORILA), volumes 1 (Tablettes éditées avant 1970) et 3 (Tablettes, nodules et rondelles éditées en 1975 et 1976). Études crétoises, XX1 (École française d'Athènes, 1976)', Revue de philologie, de littérature et d'histoire anciennes LIII:1, 129-32

Firth, R.J. (1992-1993), 'A statistical analysis of the Greekness of men's names on the Knossos Linear B tablets', Minos 27-28, 83-100

--- (1996-1997), 'The find-places of the tablets from the palace of Knossos', Minos 31-32, 7122

--- (2000-2001a), 'Evans' missing batch of Linear B tablets', Minos 35-36, 291-315

--- (2000-2001b), 'A review of the find-places of the Linear B tablets from the palace of Knossos', Minos 35-36, 53-290

--- (2011), 'Re-visiting the tablet-makers of Knossos', Pasiphae: Rivista di filologia e antichità egee V. Proceedings of the International Colloquium "The Inner Workings of Mycenaean Bureaucracy", University of Kent, Canterbury, 19-21 September 2008, $81-94$

--- (2014), 'Considering the population statistics of the sheep listed in the East-West Corridor archive at Knossos', in Nakassis, D., Gulizio, J. \& James, S.A. (eds) KE-RAME-JA. Studies Presented to Cynthia W. Shelmerdine, Prehistory Monographs 46 (Philadelphia: INSTAP Academic Press), 293-312 
Firth, R.J., \& Melena, J.L. (1998-1999), 'Identifying the Linear B tablets from the Arsenal and Little Palace at Knossos', in Bennet, J. \& Driessen, J. (eds) A-na-qo-ta. Studies presented to J.T. Killen, Minos 33-34 (Salamanca: Universidad de Salamanca), 107-34

--- (2000-2001), 'The Knossos tablets: genesis of the 5000-series', Minos 35-36, 315-56

--- (2002-2003), '22 joins and quasi-joins of fragments of LB tablets from Knossos', Minos $37-38,113-20$

--- (2008), 'The Knossos Linear B tablets: genesis of the listing of the later fragments', in Sacconi, A., Del Freo, M., Godart, L. \& Negri, M. (eds) Colloquium Romanum. Atti del XII colloquio internazionale di micenologia, Roma 20-25 febbraio 2006 vol. I, Pasiphae I (Pisa/Roma: Fabrizio Serra), 281-307

Firth, R.J., \& Nosch, M.-L.B. (2002-2003), 'Scribe 103 and the Mycenaean textile industry at Knossos: the Lc(1) and Od(1)-sets', Minos 37-38, 121-41

Foster, E.D. (1977), 'An administrative department at Knossos concerned with perfumery and offerings', Minos 16, 19-51

Furnée, E.J. (1972), Die wichtigsten konsonantischen Erscheinungen des Vorgriechischen (The Hague/Paris: Mouton)

Furumark, A. (1954), Ägäische Texte in griechischer Sprache (Gotoburgi: Elanders Boktr.) [Eranos LI-LII]

Galaty, M.L. (2007), 'Wealth ceramics, staple ceramics: pots and the Mycenaean palaces', in Galaty, M.L. \& Parkinson, W.A. (eds) Rethinking Mycenaean Palaces II: Revised and Expanded Second Edition, Monograph 60 (Los Angeles: Cotsen Institute of Archaeology, University of California), 74-86

Gallavotti, C. (1956a), Documenti e struttura della greco nell'età micenea (Roma: Edizioni dell'Ateneo)

--- (1956b), 'Il segno della luna nel sillabario miceneo', Rivista di filologia e di istruzione classica 4, 398-411

--- (1956c), 'La triade lesbia in un testo miceneo', Rivista di filologia e di istruzione classica $34: 3,225-36$ 
--- (1957a), 'Demetra micenea', Parola del passato LV, 241-9

--- (1957b), 'Labyrinthos', Parola del passato LIV, 162-76

--- (1957c), 'Nomi di colori in miceneo', Parola del passato LII, 5-22

--- (1958a), 'Il carattere eolico del greco miceneo', Rivista di filologia e di istruzione classica XXXVI, 113-33

--- (1958b), 'Le nom du cheval et les labiovélaires en mycénien', in Atti del $2^{\circ}$ colloquio internazionale di studi minoico-micenei. Pavia, 1-5 IX 1958, Athenaeum. Studi periodici di letteratura e storia dell'antichità XLVI - Nuova Serie XXXVI - Fasc. IV (Pavia: Athenaeum), 75-88 [369-82]

--- (1958c), ‘Note brevi di filologia micenea', Studi italiani di filologia classica 30:1, 52-72

--- (1959), 'Recensione: Michel Lejeune, Mémoires de philologie mycénienne (Première série 1955-1957), Centre national de la recherche scientifique, Paris 1958, pp.402', Rivista di filologia e di istruzione classica 37, 163-8

--- (1961), 'Le origini micenee dell'istituto fraterico', Parola del passato 16, 20-39

García Ramón, J.L. (2006), 'Zu den Personennamen der neuen Texte aus Theben', in Deger-Jalkotzy, S. \& Panagl, O. (eds) Die neuen Linear B-Texte aus Theben. Ihr Aufschlusswert für die mykenische Sprache und Kultur. Akten des internationalen Forschungskolloquiums an der Österreichischen Akademie der Wissenschaften, 5.-6. Dezember 2002 (Wien: Verlag der Österreichischen Akademie der Wissenschaften), $37-52$

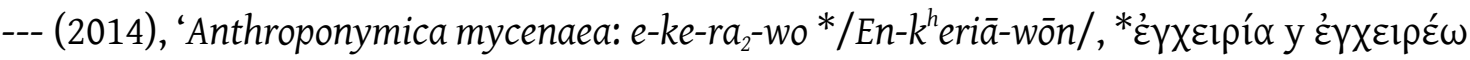

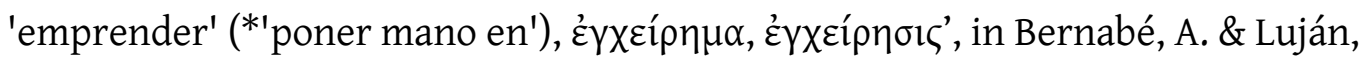
E.R. (eds) Donum Mycenologicum. Mycenaean Studies in Honour of Francisco Aura Jorro, Bibliothèque des Cahiers de l'Institut de Linguistique de Louvain 131 (Louvain-laNeuve/Walpole, MA: Peeters), 35-49

--- (2015), 'From the files of a new Mycenaean grammar' (Paper read at Aegean Scripts. 14th International Colloquium on Mycenaean Studies, held in Copenhagen, 2-5 September 2015) 
Gebhard, R., \& Rieder, K.H. (2002), 'Zwei bronzezeitliche Bernsteinobjekte mit Bild- und Schriftzeichen aus Bernstorf (Lkr. Freising)', Germania 80, 115-33

Georgiev, V. (1955), Slovar' krito-mikenskix nadpisej (Lexique des inscriptions crétomycéniennes) (Sofija: Izd-vo Bolgarskoĭ akademii nauk)

--- (1956), 'La valeur phonétique de quelques signes du syllabaire créto-mycénien B', in Lejeune, M. (ed.) Études mycéniennes. Actes du colloque international sur les textes mycéniens (Gif-sur-Yvette, 3-7 avril 1956) (Paris: Centre national de la recherche scientifique), 51-81

Godart, L. (1968), 'ku-pi-ri-jo dans les textes mycéniens', Studi micenei ed egeo-anatolici 5, 64-70

--- (1969), 'La série Fh de Cnossos', Studi micenei ed egeo-anatolici 8, 39-65

--- (1974), 'La signification de i-jo et le role de pi-ma-na-ro en KN V 1523', Parola del passato $29,271-7$

--- (1984), 'Du linéaire A au linéaire B', in Aux origines de l'hellénisme: la Crète et la Grèce: hommage à Henri van Effenterre, présenté par le Centre G. Glotz, Histoire ancienne et médiévale 15 (Paris: Publications de la Sorbonne), 121-8

--- (2009), 'I due scribi della tavoletta Tn 316', Pasiphae: Rivista di filologia e antichità egee III, 99-115

--- (2012), 'Du nouveau à l'horizon du linéaire B', in Carlier, P., de Lamberterie, C., Egetmeyer, M., Guilleux, N. et al. (eds) Études mycéniennes 2010: actes du XIIIe colloque international sur les textes égéens: Sèvres, Paris, Nanterre, 20-23 septembre 2010, Biblioteca di "Pasiphae" X (Pisa/Roma: Fabrizio Serra), 79-106

Godart, L., Killen, J.T., Kopaka, C., Melena, J.L. et al. (1990-1991), ‘501 raccords et quasiraccords de fragments dans les tablettes de Cnossos post-KT V', Minos 25-26, 373412

Godart, L., Kopaka, C., Melena, J.L., \& Olivier, J.-P. (1992-1993), '175 raccords de fragments dans les tablettes de Knossos', Minos 27-28, 55-70

Godart, L., \& Tzedakis, Y. (1989), 'La storia della lineare B e le scoperte di Armenoi e La Canea', Rivista di filologia e di istruzione classica 117, 385-409 
--- (1991), 'Les noveaux textes en linéaire B de La Canée’, Rivista di filologia e di istruzione classica 119:2, 129-49

--- (1992), Témoignages archéologiques et épigraphiques en Crète occidentale du Néolithique au Minoen Récent III B, Istituto per gli studi micenei ed egeo-anatolici: Incunabula Graeca XCIII (Roma: Gruppo editoriale internazionale)

--- (1995), 'La chute de Cnossos, le royaume de Kydonia et le scribe 115', Bulletin de correspondance hellénique 119:1, 27-33

Grammont, M. (1946), Traité de phonétique, 3rd edn. (Paris: Librairie Delagrave)

Greco, A. (2012), 'I censimenti ovini (classe D-) e la cronologia degli archivi di Cnosso', Pasiphae: Rivista di filologia e antichità egee VI, 11-46

Grumach, E. (1962), 'Mitteilungen: Linear A auf dem Festland', Kadmos 1:1, 85-6

--- (1963), ‘Zur Lesung der Knossos-Täfelchen', Kadmos 2:2, 156-66

Gulizio, J. (2008), 'Mycenaean religion at Knossos', in Sacconi, A., Del Freo, M., Godart, L. \& Negri, M. (eds) Colloquium Romanum. Atti del XII colloquio internazionale di micenologia, Roma 20-25 febbraio 2006 vol. I, Pasiphae I (Pisa/Roma: Fabrizio Serra), 351-8

Hajnal, I. (1993), 'Neue Aspekte zur Rekonstruktion des frühgriechischen Phonemsystems', Indogermanische Forschungen 98, 108-29

--- (1995), Studien zum mykenischen Kasussystem, Untersuchungen zur indogermanischen Sprach- und Kulturwissenschaft. Neue Folge 7 (Berlin: de Gruyter)

Hallager, B.P., \& Hallager, E. (2015), 'When the saints come marching in?', in Kaiser, I., Kouka, O. \& Panagiotopoulos, D. (eds) Ein Minoer im Exil. Festschrift für Wolf-Dietrich Niemeier, Universitätsforschungen zur prähistorischen Archäologie (aus dem Institut für klassische Archäologie der Universität Heidelberg) 270 (Bonn: Dr. Rudolf Habelt Verlag GmbH), 99-124

Hallager, E. (1977), The Mycenaean palace at Knossos: evidence for final destruction in the III B period 1 (Stockholm: Medelhavsmuseet) 
--- (2011), 'The Linear B inscriptions and potter's marks', in Hallager, E. \& Hallager, B.P. (eds) The Greek-Swedish Excavations at the Agia Aikaterini Square Kastelli, Khania, 19701987 and 2001: Results of the Excavations under the Direction of Yannis Tzedakis and CarlGustaf Styrenius vol. 4.1 The late Minoan IIIB:1 and IIIA:2 settlements [Text]. -- 4.2. The late Minoan IIIB:1 and IIIA:2 settlements [Plates]. Skrifter utgivna av Svenska institutet i Athen/Acta Instituti Atheniensis Regni Sueciae (Stockholm: Svenska Institutet i Athen), 415-28; Plates 270-79

--- (2012), 'Literacy at Petras and three hitherto unpublished Linear A inscriptions', in Tsipopoulou, M. (ed.) Petras, Siteia. 25 Years of Excavations and Studies. Acts of a TwoDay Conference held at the Danish Institute of Athens, 9-10 October 2010, Monographs of the Danish Institute at Athens 16 (Athens: Danish Institute at Athens; Aarhus University Press), 265-73

--- (2015), 'LM IIIB Linear B at Knossos and Khania' (Paper read at Aegean Scripts. 14th International Colloquium on Mycenaean Studies, held in Copenhagen, 2-5 September 2015)

Hallager, E., \& Hallager, B.P. (eds.) (2011), The Greek-Swedish excavations at the Agia Aikaterini Square Kastelli, Khania, 1970-1987 and 2001: results of the excavations under the direction of Yannis Tzedakis and Carl-Gustaf Styrenius, Skrifter utgivna av Svenska institutet i Athen/Acta Instituti Atheniensis Regni Sueciae 47 (Stockholm: Svenska Institutet i Athen), vol. v.4.1. The late Minoan IIIB:1 and IIIA:2 settlements [Text]. -- v.4.2. The late Minoan IIIB:1 and IIIA:2 settlements [Plates].

Hallager, E., Vlasakis, M., \& Hallager, B.P. (1992), 'New Linear B tablets from Khania', Kadmos 31:1, 61-87

Hallager, E., Vlasakis, M., \& Markoulakis, S. (1991), 'New and hitherto unpublished Linear A inscriptions from Kastelli, Khania', Kadmos 30:1, 34-41

Halstead, P. (1995), 'Late Bronze Age grain crops and Linear B ideograms *65, *120, *121', Annual of the British School at Athens 90, 229-34

Harding, A.F. (2007), 'Interconnections between the Aegean and continental Europe in the Bronze and Early Iron Ages: moving beyond scepticism', in Galanaki, I., Tomas, H., Galanakis, Y. \& Laffineur, R. (eds) Between the Aegean and Baltic Seas: Prehistory across Borders. Proceedings of the International Conference Bronze and Early Iron Age Interconnections and Contemporary Developments between the Aegean and the Regions of the Balkan Peninsula, Central and Northern Europe. University of Zagreb, 11-14 
April 2005, Aegaeum 27 (Liège/Austin: Histoire de l'art et archéologie de la Grèce antique; Program in Aegean Scripts and Prehistory), 47-56

Haskell, H.W. (1983), 'From palace to town administration: the evidence of coarse-ware stirrup jars', in Krzyszkowska, O. \& Nixon, L. (eds) Minoan Society. Proceedings of the Cambridge Colloquium 1981 (Bristol: Bristol Classical Press), 121-8

--- (1986), ‘Were LM IIIB inscribed stirrup-jars palatial?', Kadmos 25, 85-6

Heubeck, A. (1971a), 'Amphiaraos', Die Sprache. Zeitschrift für Sprachwissenschaft XVII:1, 822

--- (1971b), 'Zur s- und z-Reihe in Linear B', Kadmos 10:2, 113-24

--- (1979), 'Remarks on the sign-doublets $r \mathrm{r}_{2}, r a_{2}, t a_{2}$ ', in Risch, E. \& Mühlestein, H. (eds) Colloquium Mycenaeum. Actes du sixième colloque international sur les textes mycéniens et égéens tenu à Chaumont-sur-Neuchâtel du 7 au 13 septembre 1975 (NeuchâtelGenève: Université de Neuchâtel/Droz), 239-58

Hiller, S. (2011), 'Mycenaean religion and cult', in Duhoux, Y. \& Morpurgo Davies, A. (eds) A Companion to Linear B. Mycenaean Greek Texts and their World vol. II, Bibliothèque des Cahiers de l'Institut de Linguistique de Louvain 127 (Louvain-la-Neuve: Peeters), 169-212

Hirschfeld, N. (1990 [unpublished]), 'Incised marks on Late Helladic and Late Minoan III pottery', MA thesis, Texas A\&M University, http://nautarch.tamu.edu/Theses/pdf-files/Hirschfeld-MA1990.pdf

Hocker, F., \& Palaima, T.G. (1990-1991), 'Late Bronze Age Aegean ships and the Pylos tablets Vn 46 and Vn 879', Minos 25-26, 297-318

Hoffmann, O. (1893), Die griechischen Dialekte in ihrem historischen Zusammenhange vol. 2 Der nord-achäische Dialekt (Göttingen: Vandenhoeck und Ruprecht)

Hood, M.S.F. (1965), '"Last palace" and "reoccupation" at Knossos', Kadmos 4:1, 16-44

Hughes-Brock, H. (2011), 'Exotic materials and objects sent to - and from? - the Bronze Age Aegean. Some recent work and some observations', in Vianello, A. (ed.) Exotica in the Prehistoric Mediterranean (Oxford/Oakville: Oxbow Books), 99-114 


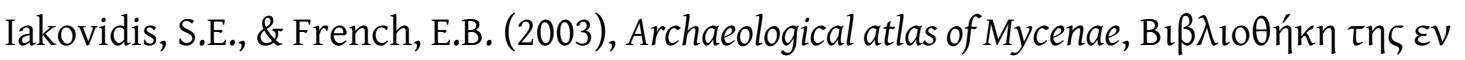

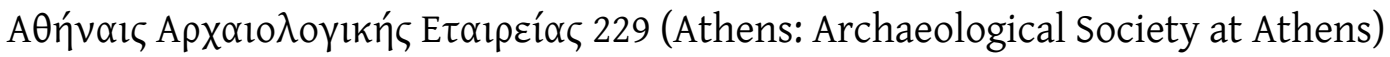

Iakovidis, S.E., Godart, L., \& Sacconi, A. (2012), 'Les inscriptions en linéaire B découvertes dans la 'Maison de Petsas' à Mycènes', Pasiphae: Rivista di filologia e antichità egee VI, 47-57

Ilievski, P. (1992), 'Observations on the personal names from the Knossos D tablets', in Olivier, J.-P. (ed.) Mykenaïka: actes du IXe Colloque international sur les textes mycéniens et égéens organisé par le Centre de l'Antiquité Grecque et Romaine de la Fondation Hellénique des Recherches Scientifiques et l'École française d'Athènes (Athènes, 2-6 octobre 1990), Bulletin de correspondance hellénique. Supplément 25 (Athens: École française d'Athènes), 321-50

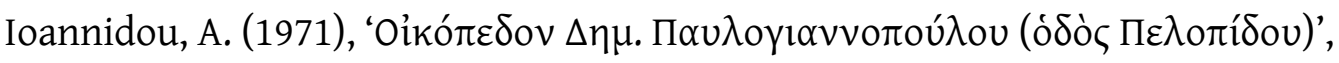

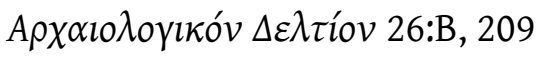

Jackson, C.M., \& Wager, E.C. (2011), 'Glass in the Aegean Bronze Age: value, meaning and status', in Vianello, A. (ed.) Exotica in the Prehistoric Mediterranean (Oxford/Oakville: Oxbow Books), 115-23

James, S.A. (2002-2003), 'The Thebes tablets and the Fq series: a contextual analysis', Minos 37-38, 397-418

Janda, M. (1986), 'Zur Lesung des Zeichens *22 von Linear B', Kadmos 25, 44-8

--- (1988), 'Zur Deutung des Zeichens *47 von Linear B', Minos 23, 107-16

Janko, R. (1987), 'Linear A and the direction of the earliest Cypro-Minoan writing', Minos 20-22, 311-18

Jasink, A.M. (1990-1991), 'Funzionarie lavoranti nel palazzo di Pilo', Minos 25-26, 203-44

--- (2013), 'The Linear B sign *34 and its likely connections with former Cretan iconography and writings', in Graziadio, G., Guglielmino, R., Lenuzza, V. \& Vitale,

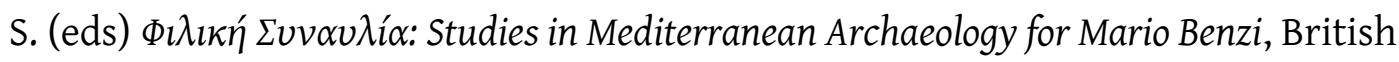
Archaeological Reports 2460 (Oxford: Archaeopress), 221-7

Jiménez Delgado, J.M. (2004), 'El grupo consonántico tw en griego micénico', Studi micenei ed egeo-anatolici XLVI:1, 15-24 
--- (2008), 'La situación de *h en griego micénico', Kadmos 47:1-2, 73-90

--- (2011), 'Distribucion y uso de los signos *76 $\left(\mathrm{ra}_{2}\right)$ y ${ }_{68}\left(\mathrm{ro}_{2}\right)$ en Lineal B', in Carande Herrero, R. \& López-Cañete Quiles, D. (eds) Pro Tantis Redditur: Homenaje a Juan Gil en Sevilla (Zaragoza: Libros Pórtico), 31-42

--- (2012), 'El genitivo singular masculino en - $a$ del griego micénico y el nominativo de rúbrica', Kadmos 51:1, 51-70

--- (2013), 'A note on the Mycenaean thematic genitive ending of month names', Indogermanische Forschungen 118, 111-24

Judson, A.P. (2013), ‘The Linear B inscribed stirrup jars’, Kadmos 52:1, 69-110

Karetsou, A., Godart, L., \& Olivier, J.-P. (1985), 'Inscriptions en linéaire A du sanctuaire de sommet minoen du Mont Iouktas', Kadmos 24:1-2, 89-147

Karnava, A. (2000 [unpublished]), 'The Cretan Hieroglyphic script of the second millenium BC: description, analysis, function and decipherment perspectives', $\mathrm{PhD}$ thesis, Faculté de Philosophie et Lettres, Université Libre de Bruxelles

Killen, J.T. (1964), 'The wool industry of Crete in the Late Bronze Age', Annual of the British School at Athens 59, 1-15

--- (1966), 'The abbreviation tu on Knossos woman tablets', Živa Antika 16, 207-12

--- (1975), 'Correspondence, 1 May 1975', in Bennett, E.L. (ed.) Nestor (Wisconsin: Institute for Research in the Humanities), 979

--- (1979), 'The Knossos Ld(1) tablets', in Risch, E. \& Mühlestein, H. (eds) Colloquium Mycenaeum. Actes du sixième colloque international sur les textes mycéniens et égéens tenu à Chaumont-sur-Neuchâtel du 7 au 13 septembre 1975 (Neuchâtel-Genève: Université de Neuchâtel/Droz), 151-82

--- (1983a), 'Mycenaean possessive adjectives in -e-jo', Transactions of the Philological Society $81: 1,66-99$ 
--- (1983b), 'TA and DA', in Oliva, P. \& Frolíková, A. (eds) Concilium Eirene XVI. Proceedings of the 16th International Eirene Conference, Prague 31.8-4.9.1982 vol. 3 (Prague: Kabinet pro studia řecká, římská a latinská ČSAV), 121-6

--- (1992), 'Observations on the Thebes sealings', in Olivier, J.-P. (ed.) Mykenaïka: actes du IXe Colloque international sur les textes mycéniens et égéens organisé par le Centre de l'Antiquité Grecque et Romaine de la Fondation Hellénique des Recherches Scientifiques et l'École française d'Athènes (Athènes, 2-6 octobre 1990), Bulletin de correspondance hellénique. Supplément 25 (Athens: École française d'Athènes), 365-80

--- (1995), 'Some further thoughts on "collectors"', in Laffineur, R. \& Niemeier, W.-D. (eds) Politeia. Society and State in the Aegean Bronze Age. Proceedings of the 5 th International Aegean Conference / 5 e Rencontre égéenne internationale, University of Heidelberg, Archäologisches Institut, 10-13 April 1994 vol. I, Aegaeum 12 (Liège/Austin: Histoire de l'art et archéologie de la Grèce antique; Program in Aegean Scripts and Prehistory), 213-26

--- (1999), 'Some observations on the new Thebes tablets', Bulletin of the Institute of Classical Studies 43, 217-19

--- (2000), ‘Two notes on Linear B’, Živa Antika 50, 141-8

--- (2003), 'Pylos tablet Va 482', in Duhoux, Y. (ed.) Briciaka. A Tribute to W.C. Brice, Cretan Studies 9 (Amsterdam: Adolf M. Hakkert), 63-76

--- (2004), 'Wheat, barley, flour, olives and figs on the Linear B tablets', in Halstead, P. \& Barrett, J.C. (eds) Food, Cuisine and Society in Prehistoric Greece, Sheffield Studies in Aegean Archaeology 5 (Oxford: Oxbow Books), 155-73

--- (2006), 'Thoughts on the functions of the new Thebes tablets', in Deger-Jalkotzy, S. \& Panagl, O. (eds) Die neuen Linear B-Texte aus Theben. Ihr Aufschlusswert für die mykenische Sprache und Kultur. Akten des internationalen Forschungskolloquiums an der Österreichischen Akademie der Wissenschaften, 5.-6. Dezember 2002 (Wien: Verlag der Österreichischen Akademie der Wissenschaften), 79-110

--- (2007), 'Critique: a view from the tablets', in Galaty, M.L. \& Parkinson, W.A. (eds) Rethinking Mycenaean Palaces II: Revised and Expanded Second Edition, Monograph 60 (Los Angeles: Cotsen Institute of Archaeology, University of California), 114-17

--- (2008), 'A note on the Knossos Mc tablets', Faventia 30:1-2, 47-52 
--- (2015), 'Conservatism and innovation in the Linear B inscriptions on stirrup jars', in Weilhartner, J. \& Ruppenstein, F. (eds) Tradition and Innovation in the Mycenaean Palatial Polities. Proceedings of an International Symposium held at the Austrian Academy of Sciences, Institute for Oriental and European Archaeology, Aegean and Anatolia Department, Vienna, 1-2 March 2013, Mykenische Studien 34 (Wien: Verlag der Österreichischen Akademie der Wissenschaften), 155-66

Killen, J.T., \& Olivier, J.-P. (1968), '155 raccords de fragments dans les tablettes de Cnossos', Bulletin de correspondance hellénique 92:1, 115-41

Kopaka, K. (1989), 'Une nouvelle inscription en linéaire A de Zakros', Kadmos 28:1-2, 7-13

Kyriakidis, E. (1996-1997), 'Some aspects of the role of scribes in Pylian palace administration', Minos 31-32, 201-29

--- (2007), 'Phonetic attributions of undeciphered characters: the case of sign *56', Cambridge Classical Journal 53, 202-28

--- (2011), 'The smell of big cheese: perfume production and the differing spheres of influence of high scribes $\mathrm{H} 1$ and $\mathrm{H} 2$ at Pylos', Pasiphae: Rivista di filologia e antichità egee V. Proceedings of the International Colloquium "The Inner Workings of Mycenaean Bureaucracy", University of Kent, Canterbury, 19-21 September 2008, 127-39

Landau, O. (1958), Mykenisch-Griechische Personennamen, Studia Graeca et Latina Gothoburgensia 7 (Göteborg: Almqvist \& Wiksell)

Lee, D.J.N. (1962 [unpublished]), 'Place-names in the Knossos tablets' (Mycenaean Epigraphy Room, Faculty of Classics, Cambridge)

Lejeune, M. (1956), 'Les documents pyliens des séries Na, Ng, Nn', in Lejeune, M. (ed.) Études mycéniennes. Actes du colloque international sur les textes mycéniens. Gif-surYvette, 3-7 avril 1956 (Paris: Centre national de la recherche scientifique), 137-65

--- (1958a), 'Addenda', in Lejeune, M. (ed.) Mémoires de philologie mycénienne. Première série (1955-1957) (Paris: Centre national de la recherche scientifique), 331-43 
--- (1958b), 'Coup d'oeil sur le système graphique', in Lejeune, M. (ed.) Mémoires de philologie mycénienne. Première série (1955-1957) (Paris: Centre national de la recherche scientifique), 318-30

--- (1958c), 'Essais de philologie mycénienne, 1. État de la recherche 2. Inventaires de roues', in Lejeune, M. (ed.) Mémoires de philologie mycénienne. Première série (19551957) (Paris: Centre national de la recherche scientifique), 17-44 [Revue de philologie XXIX (1955):147-71]

--- (1958d), 'La désinence - $\varphi$ ı en mycénien', in Lejeune, M. (ed.) Mémoires de philologie mycénienne. Première série (1955-1957) (Paris: Centre national de la recherche scientifique), 157-84 [Bulletin de la Société de Linguistique LII (1957):170-201]

--- (1958e), 'Nouvelles remarques sur l'identification des signes syllabiques rares', in Lejeune, M. (ed.) Mémoires de philologie mycénienne. Première série (1955-1957) (Paris Centre national de la recherche scientifique), 203-17

--- (1958f), 'Sur les signes notant des syllabes à groupe consonantique initial', in Lejeune, M. (ed.) Mémoires de philologie mycénienne. Première série (1955-1957) (Paris: Centre national de la recherche scientifique), 255-82

--- (1971a), 'Les syllabogrammes $t a_{2}$ et two', in Lejeune, M. (ed.) Mémoires de philologie mycénienne. Deuxième série (1958-1963) (Roma: Edizioni dell'Ateneo), 327-38 [Revue de philologie XXXVI (1962):217-24]

--- (1971b), 'Note sur le nom mycenién du 'fils', in Lejeune, M. (ed.) Mémoires de philologie mycénienne. Deuxième série (1958-1963) (Roma: Edizioni dell'Ateneo), 387-90 [Nestor 269-270 (01/08/1963)]

--- (1972a), 'Contexte et interprétation', in Lejeune, M. (ed.) Mémoires de philologie mycénienne. Troisième série (1964-1968), Istituto per gli studi micenei ed egeoanatolici: Incunabula Graeca XLIII (Roma: Edizioni dell'Ateneo), 39-54 [Minos 8.2 (1967):100-114]

--- (1972b), 'Doublets et complexes', in Lejeune, M. (ed.) Mémoires de philologie mycénienne. Troisième série (1964-1968), Istituto per gli studi micenei ed egeo-anatolici: Incunabula Graeca XLIII (Roma: Edizioni dell'Ateneo), 89-104 [L.R. Palmer \& J. Chadwick (eds), Proceedings of the Cambridge Colloquium on Mycenaean Studies (Cambridge, 1966):135-49] 
--- (1972c), 'Le génitif singulier thématique’, in Lejeune, M. (ed.) Mémoires de philologie mycénienne. Troisième série (1964-1968), Istituto per gli studi micenei ed egeoanatolici: Incunabula Graeca XLIII (Roma: Edizioni dell'Ateneo), 11-20 [Revue de philologie XXXIX (1965):14-20]

--- (1972d), 'Les diphthongues in -i à Pylos', in Lejeune, M. (ed.) Mémoires de philologie mycénienne. Troisième série (1964-1968), Istituto per gli studi micenei ed egeoanatolici: Incunabula Graeca XLIII (Roma: Edizioni dell'Ateneo), 79-88 [Revue de philologie XXXIX (1965):21-7]

--- (1972e), 'Peut-on lire au- pour 85-?', in Lejeune, M. (ed.) Mémoires de philologie mycénienne. Troisième série (1964-1968), Istituto per gli studi micenei ed egeoanatolici: Incunabula Graeca XLIII (Roma: Edizioni dell'Ateneo), 179-99 [Studi micenei ed egeo-anatolici I (1966):9-28]

--- (1972f), Phonétique historique du mycénien et du grec ancien, Tradition de l'humanisme IX (Paris: Éditions Klincksieck)

--- (1997a), 'Les syllabogrammes B et leur translitération', in Lejeune, M. (ed.) Mémoires de philologie mycénienne. Quatrième série (1969-1996), Istituto per gli studi micenei ed egeo-anatolici: Incunabula Graeca XCIX (Rome: Istituti editoriali e poligrafici internazionali), 25-52 [M.S. Ruipérez (ed.), Acta Mycenaea. Proceedings of the Fifth International Colloquium on Mycenaean Studies, held in Salamanca, 30 March - 3 April 1970. I: Minutes, Resolutions \& Reports, Minos XI (1972):73-98]

--- (1997b), 'Pré-mycénien et proto-mycénien', in Lejeune, M. (ed.) Mémoires de philologie mycénienne. Quatrième série (1969-1996), Istituto per gli studi micenei ed egeoanatolici: Incunabula Graeca XCIX (Roma: Istituti editoriali e poligrafici internazionali), 197-212 [Bulletin de la Société de Linguistique LXXI (1976):193206]

Lejeune, M., \& Godart, L. (1997), 'Le syllabogramme *56 dans le linéaire B thébain', in Lejeune, M. (ed.) Mémoires de philologie mycénienne. Quatrième série (1969-1996), Istituto per gli studi micenei ed egeo-anatolici: Incunabula Graeca XCIX (Rome: Istituti editoriali e poligrafici internazionali), 263-70 [Rivista di filologia e di istruzione classica CXXIII (1995):272-7]

Leukart, A. (1992), 'Les signes *76 ( $\mathrm{ra}_{2}$, "rja") et *68 ( $\mathrm{ro}_{2}$, "rjo") et le nom du grand prêtre de Poséidon (sinon du roi) à Pylos', in Olivier, J.-P. (ed.) Mykenaïka: actes du IXe Colloque international sur les textes mycéniens et égéens organisé par le Centre de 
l'Antiquité Grecque et Romaine de la Fondation Hellénique des Recherches Scientifiques et l'École française d'Athènes (Athènes, 2-6 octobre 1990), Bulletin de correspondance hellénique. Supplément 25 (Athens: École française d'Athènes), 387-405

Lurja, S.J. (1955), ‘Opyt čtenija pilosskix nadpisej’, Vestnik drevnej istorij 3, 8-36

Macdonald, C. (2000), 'Knossos: present and future', in Huxley, D. (ed.) Cretan Quests: British Explorers, Excavators and Historians (London: British School at Athens), 61-8

Meier-Brügger, M. (1992), Griechische Sprachwissenschaft II. Wortschatz, Formenlehre, Lautlehre, Indizes, Sammlung Göschen 2242 (Berlin/New York: de Gruyter)

--- (2003), Indo-European Linguistics, Gertmenian, C. (trans.) (Berlin/New York: de Gruyter)

Meijer, L.C. (1982), Eine strukturelle Analyse der Hagia Triada-Tafeln. Ein Betrag zur Linear AForschung, Publications of the Henri Frankfort Foundation 8 (Amsterdam: Grüner)

Meißner, T. (2008), 'Notes on Mycenaean spelling', in Sacconi, A., Del Freo, M., Godart, L. \& Negri, M. (eds) Colloquium Romanum. Atti del XII colloquio internazionale di micenologia, Roma 20-25 febbraio 2006 vol. II, Pasiphae II (Pisa/Roma: Fabrizio Serra), $507-21$

--- (2013), 'Forschungsbericht griechische Etymologie', Kratylos 58, 1-32

--- (2014), 'Linear A, Linear B: the evolution of the Bronze Age syllabaries and its consequences for our understanding of the Minoan and Greek languages' (Paper read at Aegean Seminar, University of Zagreb, 22/05/2014)

--- (forthcoming), 'Archaeology and the archaeology of the Greek language: on the origin of the Greek nouns in -عú $\varsigma^{\prime}$, in Festschrift for Anthony Snodgrass

Meißner, T., \& Steele, P.M. (forthcoming [2017]), 'Linear A and Linear B: structural and contextual concerns', in Landenius Enegren, H. \& Nosch, M.-L.B. (eds) Aegean Scripts. Proceedings of the 14th International Colloquium on Mycenaean Studies, held in Copenhagen, 2-5 September 2015, Incunabula Graeca (Roma: CNR-ISMA)

Meißner, T., \& Tribulato, O. (2002), 'Nominal composition in Mycenaean Greek', Transactions of the Philological Society 100:3, 289-330

Melena, J.L. (1972), 'On the Knossos Mc tablets', Minos 13, 29-54 
--- (1974a), 'Correspondence, 1 December 1974', in Bennett, E.L. (ed.) Nestor (Wisconsin: Institute for Research in the Humanities), 954-5

--- (1974b), 'KU-PA-RO en las tablillas de Cnoso', Emerita 42:2, 307-36

--- (1978), 'En torno a la identificación del silabograma *79 del silabario micénico', in Actas del V Congreso Español de Estudios Clásicos (Madrid 20 al 25 de abril de 1976) (Madrid: Sociedad Española de Estudios Clásicos), 751-7

--- (1983), 'Notas de filologia micenica III: el silabograma *86', Emerita 51, 262-7

--- (1985), ‘Notas de filología micénica VI: el silabograma *83’, in Rodriguez, A. \& Rodriguez, M. (eds) Serta gratulatoria in honorem Juan Régulo septuagenario oblata vol. I: Filología (La Laguna), 473-86

--- (1987a), ‘Notas de filología micénica II: ¿Qué se asienta en PY Un 1320[+]1442? ’, in Bádenas de la Pena, P., Martínez Díez, A., Martínez-Fresneda, M.E. \& Rodríguez Monescillo, E. (eds) Athlon. Satura grammatica in honorem Francisci R. Adrados vol. II (Matriti: Editorial Gredos), 613-18

--- (1987b), 'On untransliterated syllabograms *56 and *22', in Ilievski, P.H. \& Crepajac, L. (eds) Tractata Mycenaea. Proceedings of the Eighth International Colloquium on Mycenaean Studies, held in Ohrid, 15-20 September 1985 (Skopje: Macedonian Academy of Sciences and Arts), 203-32

--- (1992-1993a), '167 joins of fragments in the Linear B tablets from Pylos', Minos 27-28, 71-82

--- (1992-1993b), '244 joins and quasi-joins of fragments in the Linear B tablets from Pylos', Minos 27-28, 307-24

--- (1994-1995a), '28 joins and quasi-joins of fragments in the Linear B tablets from Pylos', Minos 29-30, 95-100

--- (1994-1995b), '133 joins and quasi-joins of fragments in the Linear B tablets from Pylos', Minos 29-30, 271-88

--- (1996-1997a), '13 joins and quasi-joins of fragments in the Linear B tablets from Pylos', Minos 31-32, 171-8 
--- (1996-1997b), '40 joins and quasi-joins of fragments in the Linear B tablets from Pylos', Minos 31-32, 159-70

--- (2000-2001), '63 joins and quasi-joins of fragments in the Linear B tablets from Pylos', Minos 35-36, 371-84

--- (2009 [unpublished]), 'Mycenaean writing [unpublished draft of Melena 2014b]' (Mycenaean Epigraphy Room, Faculty of Classics, Cambridge)

--- (2013), 'Filling gaps in the Mycenaean Linear B additional syllabary: the case of syllabogram *34', in Martínez Fernández, A., Ortega Villaro, B., Velasco López, H. \& Zamora Salamanca, H. (eds) ÁGALMA. Ofrenda desde la Filología Clásica a Manuel García Teijeiro (Valladolid: Secretariado de Publicaciones e Intercambio, Editorial Universidad de Valladolid), 207-26

--- (2014a), 'Filling gaps in the basic Mycenaean syllabary', in Bernabé, A. \& Luján, E.R. (eds) Donum Mycenologicum. Mycenaean Studies in Honour of Francisco Aura Jorro, Bibliothèque des Cahiers de l'Institut de Linguistique de Louvain 131 (Louvain-laNeuve/Walpole, MA: Peeters), 75-86

--- (2014b), 'Mycenaean writing', in Duhoux, Y. \& Morpurgo Davies, A. (eds) A Companion to Linear B: Mycenaean Greek Texts and their World vol. III, Bibliothèque des Cahiers de l'Institut de Linguistique de Louvain 133 (Louvain-la-Neuve: Peeters), 1-186

--- (forthcoming), 'On the structure of the Mycenaean Linear B syllabary. I. The untransliterated syllabograms. Preliminary report', in Proceedings of the 11th International Colloquium on Mycenaean Studies, Austin, Texas, 7th-12th May 2000 (Austin), www.academia.edu/2203598/

Meriggi, P. (1955), 'I testi micenei in trascrizione', Athenaeum 33, 64-92

Merlingen, W. (1959), Konzept einiger Linear B Indices (Wien: Verlag Notring der wissenschaftlichen Verbände Österreichs)

--- (1963), 'Linear B (und A). II. Bericht umfassend die Jahre von 1958 bis 1963', Anzeiger für die Altertumswissenschaft 16, 157-74 
Morpurgo Davies, A. (1960), 'Il genitivo miceneo e il sincretismo dei casi', Atti della Reale Accademia Nazionale dei Lincei. Rendiconti: classe di scienze morali, storiche e filologiche XV (Series 8):1-2, 33-61

--- (1972), 'Greek and Indo-European semi-consonants: Mycenaean $u$ and w', in Ruipérez, M.S. (ed.) Acta Mycenaea. Proceedings of the Fifth International Colloquium on Mycenaean Studies, held in Salamanca, 30 March - 3 April 1970, Minos 11 (Salamanca: Universidad de Salamanca), 80-121

--- (1999), 'The morphology of personal names in Mycenaean and Greek: some observations', in Deger-Jalkotzy, S., Hiller, S. \& Panagl, O. (eds) Floreant Studia Mycenaea. Akten des X. internationalen mykenologischen Colloquiums in Salzburg vom 15 Mai 1995 vol. II (Wien: Verlag der Österreichischen Akademie der Wissenschaften), 389-405

Morris, S.P. (2001), 'Potnia Aswiya: Anatolian contributions to Greek religion', in Laffineur, R. \& Hägg, R. (eds) Potnia. Deities and Religion in the Aegean Bronze Age. Proceedings of the 8th International Aegean Conference, Göteborg, Göteborg University, 12-15 April 2000, Aegaeum 22 (Liège/Austin: Histoire de l'art et archéologie de la Grèce antique; Program in Aegean Scripts and Prehistory), 423-34

Mountjoy, P.A. (1999), Regional Mycenaean Decorated Pottery vol. I, Deutsches Archäologisches Institut (Rahden/Westf.: Verlag Marie Leidorf)

Mühlestein, H. (1956), Die oka-Tafeln von Pylos. Ein mykenischer Schiffskatalog? (Basel: Selbstverlag)

Muhly, P., \& Olivier, J.-P. (2008), 'Linear A inscriptions from the Syme sanctuary, Crete',

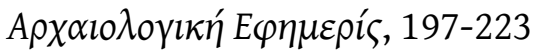

Mylonas, G.E. (1970), 'A new tablet from Mycenae, MY Fu 711', Kadmos 9:1, 48-50

Nakassis, D. (2012), 'Labor mobilization in Mycenaean Pylos', in Carlier, P., de Lamberterie, C., Egetmeyer, M., Guilleux, N. et al. (eds) Études mycéniennes 2010: actes du XIIIe colloque international sur les textes égéens: Sèvres, Paris, Nanterre, 20-23 septembre 2010, Biblioteca di "Pasiphae" X (Pisa/Roma: Fabrizio Serra), 269-83

--- (2013), Individuals and Society in Mycenaean Pylos, Mnemosyne Supplements. History and Archaeology of Classical Antiquity 358 (Leiden/Boston: Brill) 


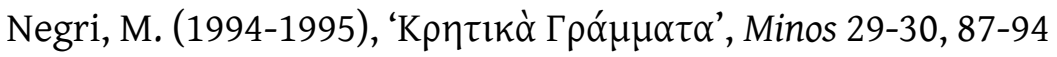

Neumann, G. (1996), 'Zur Schaffung der Zeichen *91 two und *62 pte von Linear B', in de Miro, E., Godart, L. \& Sacconi, A. (eds) Atti e memorie del secondo congresso internazionale di micenologia. Roma-Napoli, 14-20 ottobre 1991 vol. I: Filologia, Incunabula Graeca XCVIII (Roma: Gruppo editoriale internazionale), 91-9

Niemeier, W.-D. (1982), 'Mycenaean Knossos and the Age of Linear B', Studi micenei ed egeo-anatolici 23, 219-88

--- (1996), 'A Linear A inscription from Miletus (MIL Zb 1)', Kadmos 35:2, 87-99

Olivier, J.-P. (1967), Les scribes de Cnossos. Essai de classement des archives d'un palais mycénien, Istituto per gli studi micenei ed egeo-anatolici: Incunabula Graeca XVII (Roma: Edizioni dell'Ateneo)

--- (1969a), ‘En marge d'une nouvelle édition des tablettes des Mycènes', Kadmos 8, 46-53

--- (1975), 'Lire' le linéaire A?', in Bingen, J., Cambier, G. \& Nachtergael, G. (eds) Le monde grec. Hommages à Claire Préaux (Bruxelles: Editions de l'Université de Bruxelles), 441-9

--- (1984), 'Administrations at Knossos and Pylos: what differences', in Shelmerdine, C.W. \& Palaima, T.G. (eds) Pylos Comes Alive: Industry and Administration in a Mycenaean Palace (New York: Fordham University), 11-18

--- (1993), 'KN 115 = KH 115. Un même scribe à Knossos et à La Canée au MR IIIB: du soupçon à la certitude', Bulletin de correspondance hellénique 117:1, 19-33

--- (1996), 'KN 115 et KH 115: Rectification', Bulletin de correspondance hellénique 120:2, 823

--- (1997), 'The possible methods in deciphering the pictographic Cretan script', in Duhoux, Y., Palaima, T.G. \& Bennett, J. (eds) Problems in Decipherment, Bibliothèque des Cahiers de l'Institut de Linguistique de Louvain 49 (Louvain-la-Neuve: Peeters), 39-58

--- (2012a), 'Comptes rendus', in Carlier, P., de Lamberterie, C., Egetmeyer, M., Guilleux, N. et al. (eds) Études mycéniennes 2010: actes du XIIIe colloque international sur les textes égéens: Sèvres, Paris, Nanterre, 20-23 septembre 2010, Biblioteca di "Pasiphae" X (Pisa/Roma: Fabrizio Serra), 581-2 


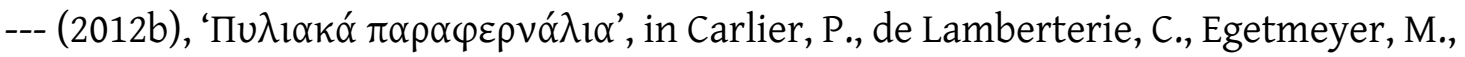
Guilleux, N. et al. (eds) Études mycéniennes 2010: actes du XIIIe colloque international sur les textes égéens: Sèvres, Paris, Nanterre, 20-23 septembre 2010, Biblioteca di "Pasiphae" X (Pisa/Roma: Fabrizio Serra), 107-22

Olivier, J.-P., \& Killen, J.T. (1992), 'Errata et corrigenda à KT ${ }^{5}$ au 04/09/90', in Olivier, J.-P. (ed.) Mykenaïka: actes du IXe Colloque international sur les textes mycéniens et égéens organisé par le Centre de l'Antiquité Grecque et Romaine de la Fondation Hellénique des Recherches Scientifiques et l'École française d'Athènes (Athènes, 2-6 octobre 1990), Bulletin de correspondance hellénique. Supplément 25 (Athens: École française d'Athènes), 457-9

Owens, G. (1991-1993), 'The untransliterated syllabograms of Linear B - 40 years on [summary of a paper delivered to the London Mycenaean Seminar, Institute of Classical Studies, 11/03/1992]', Bulletin of the Institute of Classical Studies 38, 265-6

--- (1996), 'The common origin of Cretan Hieroglyphs and Linear A', Kadmos 35:2, 105-10

Packard, D.W. (1974), Minoan Linear A (Berkeley/Los Angeles/London: University of California Press)

Palaima, T.G. (1980), 'Observations on Pylian epigraphy', Studi micenei ed egeo-anatolici 21, 193-203

--- (1981), 'On the painted linear sign from a wall at Knossos', Kadmos 20, 79-82

--- (1983), 'Evidence for the influence of the Knossian graphic tradition at Pylos', in Oliva, P. \& Frolíková, A. (eds) Concilium Eirene XVI. Proceedings of the 16th International Eirene Conference, Prague 31.8-4.9.1982 vol. III (Prague: Kabinet pro studia řecká, ř́mská a latinská ČSAV), 80-4

--- (1984), 'Inscribed stirrup jars and regionalism in Linear B Crete', Studi micenei ed egeoanatolici $25,189-203$

--- (1985), 'Appendix', in Sjöquist, K.-E. \& Åström, P. (eds) Pylos: Palmprints and Palmleaves, Studies in Mediterranean Archaeology. Pocket-book 31 (Göteborg: Paul Åströms Förlag), 99-107 
--- (1988a), 'Development of the Mycenaean writing system', in Bennett, E.L., Olivier, J.-P. \& Palaima, T.G. (eds) Texts, Tablets and Scribes: Studies in Mycenaean Epigraphy and Economy offered to Emmett L. Bennett Jr., Suplementos a Minos 10 (Salamanca: Universidad de Salamanca), 269-342

--- (1988b), The Scribes of Pylos, Istituto per gli studi micenei ed egeo-anatolici: Incunabula Graeca LXXXVII (Roma: Edizioni dell'Ateneo)

--- (1992-1993), ‘Ten reasons why KH 115 ғ KN 115’, Minos 27-28, 261-81

--- (1999), 'Kn02 - Tn 316', in Deger-Jalkotzy, S., Hiller, S. \& Panagl, O. (eds) Floreant Studia Mycenaea. Akten des X. internationalen mykenologischen Colloquiums in Salzburg vom 15 Mai 1995 vol. II (Wien: Verlag der Österreichischen Akademie der Wissenschaften), 437-62

--- (2000), 'The palaeography of Mycenaean inscribed sealings from Thebes and Pylos, their place within the Mycenaean administrative system and their links with the extra-palatial sphere', in Pini, I. (ed.) Minoisch-mykenische Glyptik: Stil, Ikonographie, Funktion. V. internationales Siegel-Symposium, Marburg, 23.-25. September 1999, Corpus der minoischen und mykenischen Siegel. Beiheft 6 (Berlin: Gebr. Mann Verlag), 219-38

--- (2000-2001), 'Review: V.L. Aravantinos, L. Godart and A. Sacconi (eds.), Les tablettes en linéaire B de la Odos Pelopidou, Édition et Commentaire (Thèbes Fouilles de la Cadmée 1) (Istituti editoriali poligrafici internazionali, Pisa/Rome 2001)', Minos 35-36, 475-86

--- (2002-2003), 'OL Zh 1: Quousque tandem?', Minos 37-38, 373-85

--- (2003), 'The inscribed bronze 'kessel' from Shaft Grave IV and Cretan heirlooms of the bronze artist named 'Aigeus' vel sim. in the Mycenaean palatial period', in Duhoux, Y. (ed.) Briciaka. A Tribute to W.C. Brice, Cretan Studies 9 (Amsterdam: Adolf M. Hakkert), 188-201

--- (2006), '*65 = FAR? or ju? and other interpretative conundra in the new Thebes tablets', in Deger-Jalkotzy, S. \& Panagl, O. (eds) Die neuen Linear B-Texte aus Theben. Ihr Aufschlusswert für die mykenische Sprache und Kultur. Akten des internationalen Forschungskolloquiums an der Österreichischen Akademie der Wissenschaften, 5.-6. Dezember 2002 (Wien: Verlag der Österreichischen Akademie der Wissenschaften), $139-48$ 
--- (2011), ‘Scribes, scribal hands and palaeography', in Duhoux, Y.\& Morpurgo Davies, A. (eds) A Companion to Linear B: Mycenaean Greek Texts and their World vol. II, Bibliothèque des Cahiers de l'Institut de Linguistique de Louvain 127 (Louvain-laNeuve: Peeters), 33-136

--- (2014), 'Pylos tablet Vn 130 and the Pylos perfume industry', in Nakassis, D., Gulizio, J. \& James, S.A. (eds) KE-RA-ME-JA. Studies Presented to Cynthia W. Shelmerdine, Prehistory Monographs 46 (Philadelphia: INSTAP Academic Press), 83-90

Palaima, T.G., \& Shelmerdine, C.W. (eds.) (1984), Pylos Comes Alive: Industry and Administration in a Mycenaean Palace (New York: Fordham University)

Palaima, T.G., \& Sikkenga, E. (1999), 'Linear A > Linear B', in Betancourt, P.P., Karageorghis, V., Laffineur, R. \& Niemeier, W.-D. (eds) Meletemata. Studies in Aegean Archaeology Presented to Malcolm H. Wiener as He Enters His 65th Year vol. II, Aegaeum 20 (Liège/Austin: Histoire de l'art et archéologie de la Grèce antique; Program in Aegean Scripts and Prehistory), 599-608

Palmer, L.R. (1954), 'M. Ventris \& J. Chadwick: Evidence for Greek dialect in the Mycenaean archives. Review', Gnomon 26:2, 65-7

--- (1955a), 'A Mycenaean calendar of offerings (PY Kn 02)', Eranos LIII, 1-13

--- (1955b), 'Observations on the Linear 'B' tablets from Mycenae', Bulletin of the Institute of Classical Studies 2, 36-45

--- (1956), 'Military arrangements for the defence of Pylos', Minos 4:2, 120-45

--- (1961), Mycenaeans and Minoans. Aegean Prehistory in the Light of the Linear B Tablets (London: Faber \& Faber)

--- (1963a), 'The find-places of the Knossos tablets', in Palmer, L.R. \& Boardman, J. (eds) On the Knossos Tablets (Oxford: Clarendon Press)

--- (1963b), The Interpretation of Mycenaean Greek Texts (Oxford: Oxford University Press)

--- (1971), ‘Mycenaean inscribed vases: Knossos’, Kadmos 10:1, 70-86 
Palmer, L.R., \& Chadwick, J. (1966), 'Report of the fourth colloquium', in Palmer, L.R. \& Chadwick, J. (eds) Proceedings of the Cambridge Colloquium on Mycenaean Studies (Cambridge: Cambridge University Press), 1-10

Palmer, R. (1992), 'Wheat and barley in Mycenaean society', in Olivier, J.-P. (ed.) Mykenaïka: actes du IXe Colloque international sur les textes mycéniens et égéens organisé par le Centre de l'Antiquité Grecque et Romaine de la Fondation Hellénique des Recherches Scientifiques et l'École française d'Athènes (Athènes, 2-6 octobre 1990), Bulletin de correspondance hellénique. Supplément 25 (Athens: École française d'Athènes), 475-97

--- (2008), 'Wheat and barley in Mycenaean society 15 years later', in Sacconi, A., Del Freo, M., Godart, L. \& Negri, M. (eds) Colloquium Romanum. Atti del XII colloquio internazionale di micenologia, Roma 20-25 febbraio 2006 vol. II, Pasiphae II (Pisa/Roma: Fabrizio Serra), 621-39

Panayotou, A. (1992), 'A nouveau les signes 'complexes' en linéaire B', in Olivier, J.-P. (ed.) Mykenaika: actes du IXe Colloque international sur les textes mycéniens et égéens organisé par le Centre de l'Antiquité Grecque et Romaine de la Fondation Hellénique des Recherches Scientifiques et l'École française d'Athènes (Athènes, 2-6 octobre 1990), Bulletin de correspondance hellénique. Supplément 25 (Athens: École française d'Athènes), 515-8

Perna, M., Kanta, A., \& Tyree, L. (2005), 'An unpublished inscription in Linear A from the Skoteino Cave, Crete (SKO Zc 1)', in Perna, M. (ed.) Studi in onore di Enrica Fiandra. Contributi di archeologia egea e vicinorientale, Studi egei e vicinorientali 3 (Napoli/Paris: de Boccard), 321-33

Perpillou, J.L. (1984), 'Les syllabogrammes *34 et *35', Studi micenei ed egeo-anatolici 25, 221-36

Petruševski, P. (1979), 'Révision de la transcription des syllabogrammes *17 (za), *20 (zo), *74 (ze) et *79 (zu?)', in Risch, E. \& Mühlestein, H. (eds) Colloquium Mycenaeum. Actes du sixième colloque international sur les textes mycéniens et égéens tenu à Chaumont-sur-Neuchâtel du 7 au 13 septembre 1975 (Neuchâtel-Genève: Université de Neuchâtel/Droz), 259-66

Pierini, R. (2014), 'Ricerche sul segno 25 del sillabario miceneo', in Bernabé, A. \& Luján, E.R. (eds) Donum Mycenologicum: Mycenaean Studies in Honour of Francisco Aura Jorro, 
Bibliothèque des Cahiers de l'Institut de Linguistique de Louvain 131 (Louvain-laNeuve/Walpole, MA: Peeters), 105-37

Piquero, J. (2015), 'Micénico pa-ra-ku-we y telas pa-ra-ku-ja: una nueva etimología en el contexto del Oriente Próximo', Aula Orientalis 33:1, 115-26

Pisani, V. (1959), 'Sul genitivo miceneo dei temi in -o-', Parola del passato LXV, 81-6

Piteros, C., Olivier, J.-P., \& Melena, J.L. (1990), 'Les inscriptions en linéaire B des nodules de Thèbes: la fouille, les documents, les possibilités d'interprétation', Bulletin de correspondance hellénique 114:1, 103-84

Plath, R. (1994), Der Streitwagen und seine Teile im frühen Griechischen. Sprachliche Untersuchungen zu den mykenischen Texten und zum homerischen Epos, Erlanger Beiträge zur Sprache, Literatur und Kunst 76 (Nürnberg: Verlag Hans Carl)

--- (2004), 'Zum Lautwert des Zeichens 63 in der Linear B-Schrift', Kadmos 42:1-2, 1-14

Platon, N., \& Stassinopoulou-Touloupa, E. (1964a), 'Ivories and Linear-B from Thebes', Illustrated London News [London, England] 5 Dec. 1964, 896-7

--- (1964b), 'Oriental seals from the Palace of Cadmus: unique discoveries in Boeotian Thebes', Illustrated London News [London, England] 28 Nov. 1964, 859-61

Pluta, K. (1996-1997), 'A reconstruction of the Archives Complex at Pylos: preliminary progress report', Minos 31-32, 231-50

Popham, M.R. (1988), 'The historical implications of the Linear B archive at Knossos dating to either c.1400 BC or 1200 BC', Cretan Studies 1, 217-28

--- (1993), 'Driessen (J.) An early destruction in the Mycenaean palace at Knossos: a new interpretation of the excavation field-notes of the south-east area of the west wing. Katholieke Universiteit Leuven, 1990 (Review)', Journal of Hellenic Studies $113,174-8$

--- (1997), 'The final destruction of the palace at Knossos: seals, sealings and pottery: a reconsideration', in Driessen, J. \& Farnoux, A. (eds) La Crète mycénienne. Actes de la Table Ronde internationale organisée par l'École française d'Athènes, 26-28 mars 1991, Bulletin de correspondance hellénique. Supplément 30 (Athens: École française d'Athènes), 375-85 


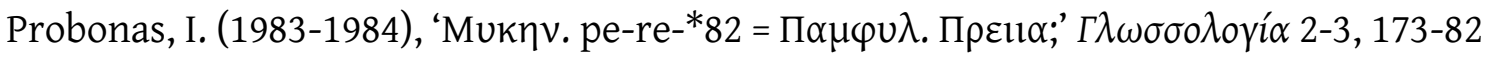

Pugliese Carratelli, G. (1957), Note su testi 'micenei', Accademia Toscana di Scienze e Lettere "La Colombaria" (Firenze: Leo S. Olschki) [Atti dell'Accademia Toscana di Scienze e Lettere "La Colombaria", 1956]

Quattordio-Moreschini, A. (1987), 'Il miceneo pe-re-*82 e la famiglia lessicale di $\pi \rho \varepsilon ́ \sigma \beta u \varsigma '$, Studi classici e orientali 37, 385-405

Raison, J. (1964), 'Note sur le signe mycénien 65’, Kadmos 3:1, 58-63

--- (1988), Le palais du second millénaire à Knossos I: le Quartier Nord, École française d'Athènes: Études crétoises XXVIII (Paris: P. Geuthner)

--- (1993), Le palais du second millénaire à Knossos II: le Front Ouest et ses magasins, École française d'Athènes: Études crétoises XXIX (Paris: de Boccard)

Raison, J., \& Pope, M. (1977), Index transnuméré du linéaire A, Bibliothèque des Cahiers de l'Institut de Linguistique de Louvain 11 (Louvain-la-Neuve: Peeters)

Risch, E. (1957), 'Mykenisch wo-wo ko-to-no', Minos 5:1, 28-34

--- (1979), 'Les consonnes palatalisées dans le grec du II ${ }^{\mathrm{e}}$ millénaire et des premiers siècles du I ${ }^{\mathrm{er}}$ millénaire', in Risch, E. \& Mühlestein, H. (eds) Colloquium Mycenaeum. Actes du sixième colloque international sur les textes mycéniens et égéens tenu à Chaumont-sur-Neuchâtel du 7 au 13 septembre 1975 (Neuchâtel-Genève: Université de Neuchâtel/Droz), 267-81

--- (1987), ‘Die mykenischen Personennamen auf -e’, in Ilievski, P. \& Crepajac, L. (eds) Tractata Mycenaea. Proceedings of the Eighth International Colloquium on Mycenaean Studies, held in Ohrid, 15-20 September 1985 (Skopje: Macedonian Academy of Sciences and Arts), 281-98

Risch, E., \& Hajnal, I. (2006), Grammatik des mykenischen Griechisch (Sprachwissenschaft Innsbruck: Institut für Sprachen und Literaturen), https://web.archive.org/web/20080514121722/http://www.uibk.ac.at/sprachenliteraturen/sprawi/mykgr.html 
Ruijgh, C.J. (1967), Études sur la grammaire et le vocabulaire du grec mycénien (Amsterdam: Adolf M. Hakkert)

--- (1972), 'Quelque hypothesès en marge des tablettes En-Ep/Eo-Eb de Pylos', Studi micenei ed egeo-anatolici 15, 91-104

--- (1979), 'Le syllabogramme *34/35 du linéaire B: valeur possible $\mathrm{ru}_{2}=1 \mathrm{lu}$ ', in Carruba, $\mathrm{O}$. (ed.) Studia mediterranea Piero Meriggi dicata (Pavia: Aurora Edizioni), 555-72

Ruipérez, M.S. (1958), 'Mykenisch Pe-re-sa $a_{2}$ "Persephone"', in Grumach, E. (ed.) Minoica. Festschrift zum 80. Geburtstag von Johannes Sundwall (Berlin: Akademie-Verlag), 35964

--- (1972a), 'Le dialecte mycénien', in Ruipérez, M.S. (ed.) Acta Mycenaea. Proceedings of the Fifth International Colloquium on Mycenaean Studies, held in Salamanca, 30 March - 3 April 1970 vol. I, Minos 11 (Salamanca: Universidad de Salamanca), 136-69

--- (1972b), 'Minutes and resolutions', in Ruipérez, M.S. (ed.) Acta Mycenaea. Proceedings of the Fifth International Colloquium on Mycenaean Studies, held in Salamanca, 30 March - 3 April 1970 vol. I, Minos 11 (Salamanca: Universidad de Salamanca), xi-xxiii

--- (1983), 'The Mycenaean name of Dionysos', in Heubeck, A. \& Neumann, G. (eds) Res Mycenaeae. Akten des VII. internationalen mykenologischen Colloquiums in Nürnberg vom 6.-10. April 1981 (Göttingen: Vandenhoeck \& Ruprecht), 408-12

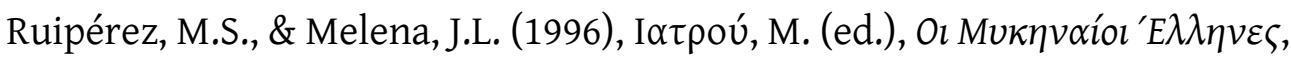

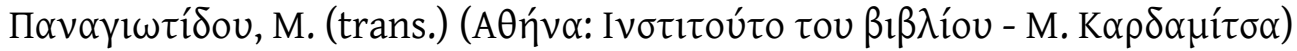

Sacconi, A. (1973), 'L'avorio nella tabella di Pilo Va 482', Minos 13:2, 173-81

--- (2012), 'Il supplemento al corpus delle iscrizioni vascolari in lineare B', in Carlier, P., de Lamberterie, C., Egetmeyer, M., Guilleux, N. et al. (eds) Études mycéniennes 2010: actes du XIIIe colloque international sur les textes égéens: Sèvres, Paris, Nanterre, 20-23 septembre 2010, Biblioteca di "Pasiphae" X (Pisa/Roma: Fabrizio Serra), 123-42

Sacconi, A., \& Cultraro, M. (2015), 'Un fragment de vase inscrit de Prinias: PRI Z 1' (Paper read at Aegean Scripts. 14th International Colloquium on Mycenaean Studies, held in Copenhagen, 2-5 September 2015) 
Sakellarakis, Y., \& Sapouna-Sakellaraki, E. (1997), Archanes. Minoan Crete in a New Light (Athens: Ammos Publications/Eleni Nakou Foundation)

Salgarella, E. (2015 [unpublished]), 'The use of the particle -qe in Mycenaean Greek', MPhil thesis, Faculty of Classics, Cambridge

Sergent, B. (1977), 'La liste de Kom El-Hetan et le Péloponnèse’, Minos 16, 126-73

Shelmerdine, C.W. (1985), The Perfume Industry of Mycenaean Pylos (Göteborg: Paul Åströms Förlag)

--- (1988), ‘'Scribal organization and administrative procedures', in Olivier, J.-P. \& Palaima, T.G. (eds) Texts, Tablets and Scribes. Studies in Mycenaean Epigraphy and Economy offered to Emmett L. Bennett, Jr., Suplementos a Minos 10 (Salamanca: Universidad de Salamanca), 343-84

--- (1997), 'Workshops and record keeping in the Mycenaean world', in Laffineur, R. \& Betancourt, P.P. (eds) TEXNH. Craftsmen, Craftswomen and Craftsmanship in the Aegean Bronze Age. Proceedings of the 6th International Aegean Conference, Philadelphia, Temple University, 18-21 April 1996 vol. II, Aegaeum 16 (Liège/Austin: Histoire de l'art et archéologie de la Grèce antique; Program in Aegean Scripts and Prehistory), 387-96

--- (1998-1999), 'The Southwestern Department at Pylos', in Bennet, J. \& Driessen, J. (eds) A-NA-QO-TA: Studies presented to J.T. Killen, Minos 33-34 (Salamanca: Universidad de Salamanca), 309-37

--- (1999), 'A comparative look at Mycenaean administrations', in Deger-Jalkotzy, S., Hiller, S. \& Panagl, O. (eds) Floreant Studia Mycenaea. Akten des X. internationalen mykenologischen Colloquiums in Salzburg vom 1-5 Mai 1995 vol. II (Vienna: Verlag der Österreichischen Akademie der Wissenschaften), 555-76

--- (2001), 'The palatial Bronze Age of the southern and central Greek mainland (with addendum: 1997-1998)', in Cullen, T. (ed.) Aegean Prehistory: a Review, American Journal of Archaeology Supplement 1 (Boston: Archaeological Instiute of America), 329-78

--- (2008), 'Backgrounds, sources, methods', in Shelmerdine, C.W. (ed.) The Cambridge Companion to the Aegean Bronze Age (Cambridge: Cambridge University Press), 1-18 
--- (2012), 'Iklaina tablet IK X 1', in Carlier, P., de Lamberterie, C., Egetmeyer, M., Guilleux, N. et al. (eds) Études mycéniennes 2010. Actes du XIIIe colloque international sur les textes égéens, Sèvres, Paris, Nanterre, 20-23 septembre 2010, Biblioteca di "Pasiphae" X (Pisa/Roma: Fabrizio Serra), 75-8

Sittig, E. (1954), 'Review of E.L. Bennett, A Minoan Linear B Index (Yale University Press, 1953)', Bibliotheca Orientalis 11, 67-71

Sjöquist, K.-E., \& Åström, P. (1985), Pylos: Palmprints and Palmleaves, Studies in Mediterranean Archaeology. Pocket-book 31 (Göteborg: Paul Åströms Förlag)

--- (1991), Knossos: Keepers and Kneaders, Studies in Mediterranean Archaeology and Literature. Pocket-book 82 (Göteborg: Paul Åströms Förlag)

Skelton, C., (2006), 'Skelton Phylogenetics', http://www.utexas.edu/research/pasp/phylo/, retrieved: 24/07/2013

--- (2008), 'Methods of using phylogenetic systematics to reconstruct the history of the Linear B script', Archaeometry 50, 158-76

--- (2011), 'Reconstructing the history of Linear B using phylogenetic systematics', Pasiphae: Rivista di filologia e antichità egee V. Proceedings of the International Colloquium "The Inner Workings of Mycenaean Bureaucracy", University of Kent, Canterbury, 19-21 September 2008, 71-80

Smith, J. (1992-1993), ‘The Pylos Jn Series', Minos 27-28, 167-260

Steele, P.M. (2014), 'The /d/, /t/, /l/ and /r/ series in Linear A and B, Cypro-Minoan and the Cypriot Syllabary', Pasiphae: Rivista di filologia e antichità egee VIII, 189-96

Steele, P.M., \& Meißner, T. (forthcoming), 'From Linear B to Linear A: the problem of the backward projection of sound values', in Steele, P.M. (ed.) Understanding Relations Between Scripts: The Aegean Writing Systems (Oxford: Oxbow Books)

Stephens, L., \& Justeson, J.S. (1978), 'Reconstructing 'Minoan' phonology: the approach from universals of language and universals of writing systems', Transactions of the American Philological Association 108, 271-84 
Survinu, C. (1968), 'La radice pel/per nella religione greca e micenea', in Atti e memorie del $1^{\circ}$ congresso internazionale di micenologia (Roma 27 settembre-3 ottobre 1967) vol. II, Incunabula Graeca XXV (Roma: Edizioni dell'Ateneo), 1059-70

Thompson, R.J.E. (2002-2003), 'Special vs. Normal Mycenaean revisited', Minos 37-38, 33770

--- (2005), ‘Two notes on Mycenaean labial stops’, Živa Antika 55, 107-15

--- (2008), 'Mycenaean non-assibilation and its significance for the prehistory of the Greek dialects', in Sacconi, A., Del Freo, M., Godart, L. \& Negri, M. (eds) Colloquium Romanum. Atti del XII colloquio internazionale di micenologia, Roma 20-25 febbraio 2006 vol. II, Pasiphae II (Pisa/Roma: Fabrizio Serra), 753-66

--- (2014), 'Mycenaean - pi and pa-ro in the light of TH Uq 434', in Bernabé Pajares, A. \& Luján, E.R. (eds) Donum Mycenologicum. Mycenaean Studies in Honour of Francisco Aura Jorro, Bibliothèque des Cahiers de l'Institut de Linguistique de Louvain 131 (Louvain-la-Neuve/Walpole, MA: Peeters), 163-78

--- (forthcoming), 'The Mycenaean writing system', in Killen, J.T. \& Morpurgo Davies, A. (eds) Documents in Mycenaean Greek, 3rd edn. (Cambridge: Cambridge University Press)

--- (forthcoming [2017]), 'The Mycenaean o-stem genitive singular in -o: a re-evaluation', in Landenius Enegren, H. \& Nosch, M.-L.B. (eds) Aegean Scripts. Proceedings of the 14th International Colloquium on Mycenaean Studies, held in Copenhagen, 2-5 September 2015 (Roma: CNR-ISMA)

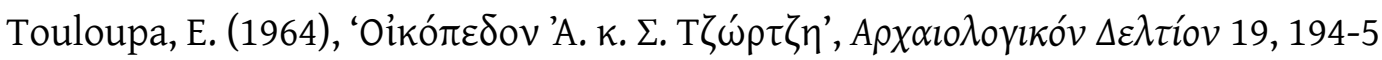

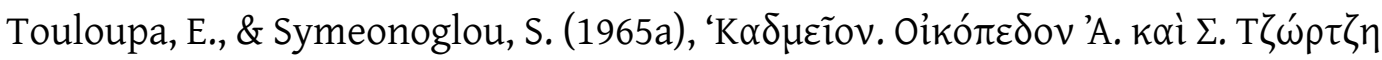

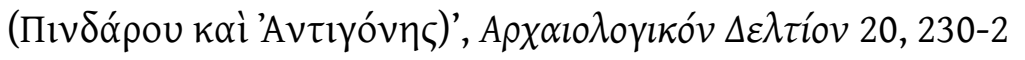

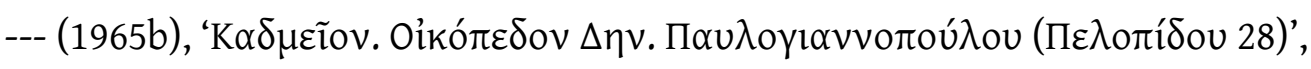

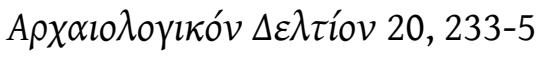

Tournavitou, I. (1995), The Ivory Houses at Mycenae (London: British School at Athens)

Tovar, A. (1958), 'El signo micenico $83=\mathrm{qe}_{2}$, , in Grumach, E. (ed.) Minoica. Festschrift zum 80. Geburtstag von Johannes Sundwall, Deutsche Akademie der Wissenschaften zu 
Berlin. Schriften der Sektion für Altertumswissenschaft 12 (Berlin: AkademieVerlag), 402-5

Tritsch, F.J. (1958), 'The women of Pylos', in Grumach, E. (ed.) Minoica. Festschrift zum 80. Geburtstag von Johannes Sundwall, Deutsche Akademie der Wissenschaften zu Berlin. Schriften der Sektion für Altertumswissenschaft 12 (Berlin: AkademieVerlag), 406-45

Tsipopoulou, M., Godart, L., \& Olivier, J.-P. (1982), 'Bol de bronze à base omibiliquée avec inscription en linéaire A de la collection K. et M. Mitsotakis', Studi micenei ed egeoanatolici $23,61-72$

Tsipopoulou, M., \& Hallager, E. (1996), 'Inscriptions with Hieroglyphics and Linear A from Petras, Siteia', Studi micenei ed egeo-anatolici 37, 7-46

--- (2010), The Hieroglyphic Archive at Petras, Siteia, Monographs of the Danish Institute at Athens 9 (Aarhus: Danish Institute at Athens)

Valério, M. (2007), 'Diktaian Master': A Minoan predecessor of Diktaian Zeus in Linear A?', Kadmos 46, 3-14

--- (2015), 'Linear A du- $\mathrm{pu}_{2}$-re, Hittite tabarna and their alleged relatives revisited', Journal of Language Relationship 13:4, 329-54

Vandenabeele, F., \& Olivier, J.-P. (1979), Les idéogrammes archéologiques du linéaire B, École française d'Athènes: Études crétoises 24 (Paris: P. Geuthner)

Varias García, C. (1993), 'Los documentos en Lineal B de Micenas. Ensayo de interpretacíon global', PhD thesis, Facultat de Filosofia i Lletres, Departament de Ciències de l'Antiguitat i de l'Edat Mitjana, Universitat Autònoma de Barcelona, Bellaterra, http://ddd.uab.cat/record/38003?ln=en

Vasilogamvrou, A. (2015), 'The West Stoa archive at Ayios Vasileios: archaeological context, chronology and preliminary study of the documents' (Paper read at Aegean Scripts. 14th International Colloquium on Mycenaean Studies, held in Copenhagen, 2-5 September 2015)

Ventris, M.G.F. (1952 [unpublished]), 'Experimental Mycenaean vocabulary 7.52’ (Mycenaean Epigraphy Room, Faculty of Classics, Cambridge) 
--- (1953), 'Mycenaean epigraphy: suggested code of practice', Bulletin of the Institute of Classical Studies 1, 3-10

Ventris, M.G.F., \& Chadwick, J. (1953), 'Evidence for Greek dialect in the Mycenaean archives', Journal of Hellenic Studies 73, 84-103

Warren, P. (1989), 'The destruction of the palace of Knossos', in Karageorghis, V. (ed.) The Civilizations of the Aegean and their Diffusion in Cyprus and the Eastern Mediterranean, 2000-600 B.C., 18-24 September 1989 (Larnaca: Pierides Foundation), 33-8

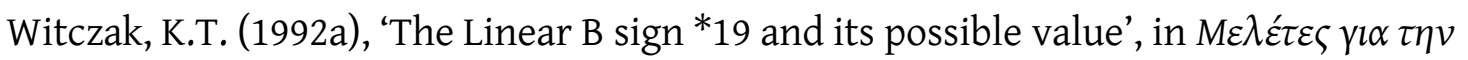

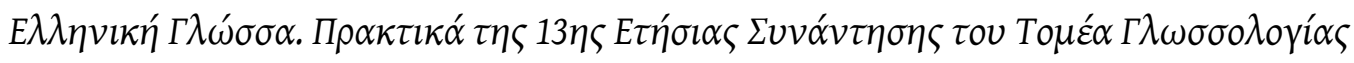

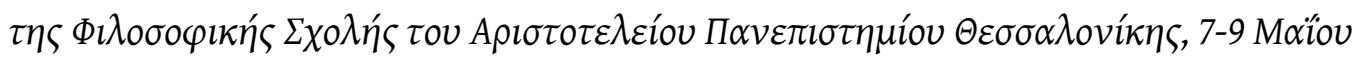
1992/ Studies in Greek linguistics. Proceedings of the 13th Annual Meeting of the Department of Linquistics, Faculty of Philosophy, Aristotle University of Thessaloniki, 7-9

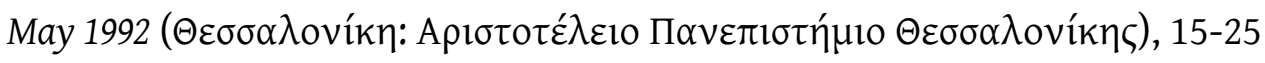

--- (1992b), ‘The phonetic value of the Linear B sign *47’, Kadmos 31:1, 88-92

--- (1993a), 'A b-series in Linear B', Kadmos 32, 162-71

--- (1993b), 'Greek Aphrodite and her I.-E. origin. With an excursus on Myc. pe-re-wa 2 and

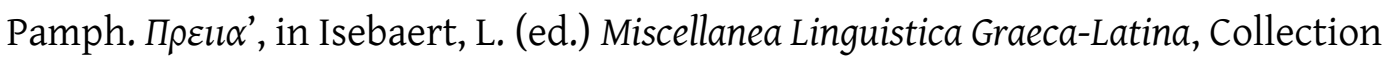
d'études classiques 7 (Namur: Société des études classiques), 115-23

--- (1994), 'The Linear B sign *76: a proposal for new value $\left(\mathrm{ri}_{2}\right)^{\prime}$, Pomoerium: studia et commentarii ad orbem classicum spectantia 1, 7-14

--- (1999), 'The Linear B sign *64 and its phonetic value (zi)', Živa Antika 49, 5-14

--- (2002-2003), 'A new Linear B sign *92 and the b-series', DO-SO-MO: Fascicula Mycenologica Polona 4-5, 125-8

--- (2011), 'Dioscuri in the Mycenaean times', DO-SO-MO: Fascicula Mycenologica Polona 9, 57-68

Woodhead, A.G. (1981), The Study of Greek Inscriptions (Cambridge: Cambridge University Press) 
Yakubovich, I. (2002), 'Labyrinth for tyrants', in Kassian, A.S. \& Sidel'tsev, A.V. (eds) Studia Linguarum 3 (Moscow: Languages of Slavonic Culture), 93-116

Younger, J.G. (1996-1997), ‘The Cretan Hieroglyphic script: a review article', Minos 31-32, $379-400$

--- (2003), 'Cretan Hieroglyphic transaction terms: 'total paid' and 'total owed', in Duhoux, Y. (ed.) Briciaka. A Tribute to W.C. Brice, Cretan Studies 9 (Amsterdam: Adolf M. Hakkert), 301-16 